\title{
Assessment of Radionuclides in the Savannah River Sith Environment Summary
}

FEB 051999

by

W. H. Carlton

Westinghouse Savannah River Company

Savannah River Site

Aiken, South Carolina 29808

DOE Contract No. DE-AC09-96SR18500

This paper was prepared in connection with work done under the above contract number with the U.S. Department of Energy. By acceptance of this paper, the publisher and/or recipient acknowledges the U. S. Government's right to retain a nonexclusive, royalty-free license in and to any copyright covering this paper, along with the right to reproduce and to authorize others to reproduce all or part of the copyrighted paper. 


\section{DISCLAIMER}

This report was prepared as an account of work sponsored by an agency of the United States Government. Neither the United States Government nor any agency thereof, nor any of their employees, makes any warranty, express or implied, or assumes any legal liability or responsibility for the accuracy, completeness, or usefulness of any information, apparatus, product, or process disclosed, or represents that its use would not infringe privately owned rights. Reference herein to any specific commercial product, process, or service by trade name, trademark, manufacturer, or otherwise does not necessarily constitute or imply its endorsement, recommendation, or favoring by the United States Government or any agency thereof. The views and opinions of authors expressed herein do not necessarily state or reflect those of the United States Government or any agency thereof.

This report has been reproduced directly from the best available copy.

Available to DOE and DOE contractors from the Office of Scientific and Technical Information, P. O. Box 62, Oak Ridge, TN 37831; prices available from (423) 576-8401.

Available to the public from the National Technical Information Service, U. S. Department of Commerce, 5285 Port Royal Road, Springfield, VA 22161. 


\section{DISCLAIMER}

Portions of this document may be illegible in electronic image products. Images are produced from the best available original document. 
WSRC-TR-98-00162

September 1998

\section{Assessment of Radionuclides in the Savannah River Site Environment- Summary (U)}



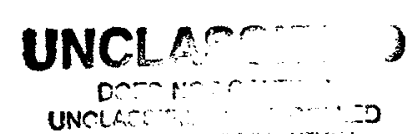

NUGLEA lionand

1008

Roviewing Dgmone Sied sow

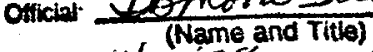

$\operatorname{lom} \frac{1162.198}{162}$

Deriative Classifier

\title{
Assessment of Radionuclides in the Savannah River Site Environment-Summary (U)
}

\author{
W. H. Carlton
}

Approved by:

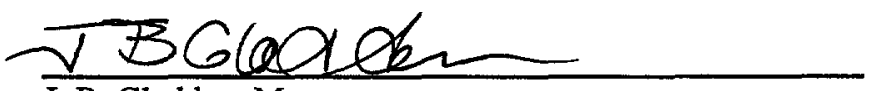

J. B. Gladden, Manager

Environmental Analysis Section

Prepared for the U.S. Department of Energy under contract no. DE-AC09-96SR18500 


\section{Executive Summary}

A series of documents has been published that assesses the impact of various radionuclides released to the environment by Savannah River Site (SRS) operations. The quantity released, the disposition of the radionuclides in the environment, and the dose to offsite individuals has been presented for activation products, carbon, cesium, iodine, plutonium, selected fission products, selected transuranics (neptunium, actinium, and curium), strontium, technetium, tritium, uranium, and the noble gases. An assessment of the impact of nonradioactive mercury also has been published.

This document summarizes the impact of radionuclide releases from SRS facilities from 1954 through 1996. The radionuclides reported here are those whose release resulted in the highest dose to people living near SRS.

Release pathways, emission control features, and annual releases to the aqueous and atmospheric environments are discussed. The releases were the result of normal operations of the reactors and separations facilities, as well as incidents that resulted in acute releases to the environment. Releases declined over the years as better controls were established and production was reduced.

The overall radiological impact of SRS radionuclide atmospheric releases from 1954 through 1996 on the offsite maximally exposed individual can be characterized by a total dose of 77 mrem. During the same period, this individual received a total dose of 15,500 mrem from non-SRS sources of ionizing radiation present in the environment. SRS aqueous releases resulted in a total dose of 140 mrem to the maximally exposed individual.

The impact of SRS radionuclide releases on offsite populations also has been evaluated. The total collective dose was estimated as 4,800 person-rem, distributed among 620,100 individuals. Using international risk factors, approximately 2.4 cancer deaths are predicted from SRS releases, while almost 100,000 fatal cancers will occur in this population from all other causes. 
Assessment of Radionuclides

the Savannah River Site Environment-Summary (U)

WSRC-TR-98-00162 
Executive Summary $i$

Chapter 1. Introduction 1-1

Chapter 2. Origin and Disposition of Radionuclides at SRS 2-1

Irradiation in Production Reactors 2-3

Radionuclides in Fuel and Targets Irradiated in Production Reactors 2-3

Other Sources Due to SRS Operations $2-4$

SRS Test Reactors 2-4

Neutron Activation Analysis 2-4

Materials Originating Offsite $2-4$

Chapter 3. Methods of Dose Calculation 3-1

Models of Radioactive Material Transport and Dose 3-3

Modeling Atmospheric Dispersion

of Radioactive Releases 3-3

MAXIGASP 3-4

POPGASP 3-4

Modeling Doses from Liquid Releases 3-5

Validation of Transport Models Using Monitoring Data 3-6

Atmospheric Releases 3-6

Liquid Releases 3-6

References for Chapter 3 3-7

Chapter 4. Releases and Doses of Individual Radionuclides 4-1

Activation Products 4-3

Phosphorus-32 4-3

Chromium-51 4-3

Cobalt-60 4-3

Zinc-65 4-3

Carbon-14 4-4 
Cesium-137 4-4

lodine-129 and lodine-131 4-5

Americium and Curium 4-5

Americium-241 4-5

Curium-244 4-5

Noble Gases, Argon-41 4-6

Plutonium 4-6

Selected Fission Products 4-6

Zirconium,Niobium-95 4-7

Ruthenium-106 4-7

Cerium-144 4-7

Strontium 4-7

Technetium 4-8

Tritium 4-8

Uranium 4-8

Site 4-8

References for Chapter 4 4-9

Chapter 5. Dose Consequences 5-1

Relationship of Dose

to Risk and Health Effects 5-3

lonizing Radiation 5-3

Cancer Risk Estimates for Atmospheric Releases 5-3

Cancer Risk Estimates for Liquid Releases 5-4

Comparisons of Doses Near SRS with Applicable Regulations 5-4

Atmospheric Releases 5-4

Liquid Releases 5-4

Summary of Dosimetric Impacts 5-4

References for Chapter 5 5-5

Additional Reading 5-6 


\section{List of Figures}

Figure 2.1. Areas Within SRS That May Be Sources of Radionuclide Releases 2-2

Figure 3.1. Simplified Pathways Between Radioactive Materials Released to the Atmosphere and Man 3-4

Figure 3.2. Simplified Pathways Between Radioactive Materials Released to Groundwater or Surface Waters and Man 3-7

\section{List of Tables}

Table 1-1. Table 1-2.

Table 3-1.

Table 3-2.

Table 3-3.

Table 4-1.

Table 4-2.

Table 4-3.

Table 4-4.

Table 4-5.

Table 4-6.

Table 4-7.

Table 4-8.

Table 4-9.

Table 4-10.

Table 4-11.

Table 4-12.

Table 4-13.

Table 4-14.

Table 4-15.

Table 4-16.

Table 4-17.

Table 4-18.

Table 4-19.

Table 4-20.

Table 4-21.

Table 4-22.

Table 4-23.

Table 4-24.

Table 4-25.

Table 4-26.

Table 4-27.

Table 4-28.

Table 4-29.

Table 4-30.

Table 4-31.
Individual Radionuclides and Corresponding RAP Document 1-2 Authors Contributing to RAP Documents 1-3

Site-Specific Parameters for Atmospheric Releases 3-5

Site-Specific Parameters for Liquid Releases 3-6

Additional Site-Specific Parameters for Liquid Releases 3-6

Liquid P-32 Releases and Dose 4-10

Liquid Cr-51 Releases and Dose 4-11

Atmospheric Co-60 Releases and Dose 4-12

Liquid Co-60 Releases and Dose 4-13

Liquid Zn-65 Releases and Dose 4-14

Atmospheric C-14 Releases and Dose 4-15

Atmospheric Cs-137 Releases and Dose 4-16

Liquid Cs-137 Releases and Dose 4-17

Atmospheric Iodine Releases and Dose 4-18.

Liquid I-131 Releases and Dose 4-19

Atmospheric Am-241 Releases and Dose 4-20

Atmospheric Cm-244 Releases and Doses 4-21

Liquid Cm-244 Releases and Dose 4-22

Atmospheric Ar-41 Releases and Dose 4-23

Atmospheric Plutonium Releases and Dose 4-24

Liquid Plutonium Releases and Dose 4-25

Liquid $\mathrm{Zr}, \mathrm{Nb}-95$ Releases and Dose 4-26

Atmospheric Ru-106 Releases and Dose 4-27

Liquid Ru-106 Releases and Dose 4-28

Liquid Ce-144 Releases and Dose 4-29

Atmospheric Strontium Releases and Dose 4-30

Liquid Sr-90 Releases and Dose 4-31

Atmospheric H-3 Releases and Dose 4-32

Liquid H-3 Releases and Dose to the Maximally

Exposed Individual 4-33

Liquid H-3 Releases and Population Dose 4-34

Atmospheric Uranium Releases and Dose 4-35

Liquid Uranium Releases and Dose 4-36

Total Dose to the Maximally Exposed Individual

from Atmospheric Releases (mrem) 4-37

Population Dose from Atmospheric Releases (person-rem) 4-38

Total Dose to the Maximally Exposed Individual

from Liquid Releases (mrem) 4-39

Total Dose to the Maximally Exposed Individual

at Beaufort-Jasper from Liquid Releases (mrem) 4-40 
Table 4-32. Total Dose to the Maximally Exposed Individual at Port Wentworth from Liquid Releases (mrem) 4-41

Table 4-33. Population Dose at Beaufort-Jasper from Liquid Releases (person-rem) 4-42

Table 4-34. Population Dose at Port Wentworth

from Liquid Releases (person-rem) 4-43

Table 4-35. Population Dose (80-km)

Table 4-36. Total Population Dose from Liquid Releases (person-rem) 4-45

Table 4-37. Total Population Dose from Releases by Radionuclide (person-rem) 4-46

Table 4-38. Percent of Population Dose Contributed by each Radionuclide $4-47$

Table 4-39. Total Population Dose by Year (person-rem) 4-48

Table 5-1. Occupational Doses 5-5 


\section{Chapter 1. Introduction}

This chapter describes a decade-long effort to examine releases of radionuclides from the Savannah River Site and their effect on the environment. 
Table 1-1. Individual Radionuclides and Corresponding RAP Document

\begin{tabular}{|c|c|}
\hline Radionuclide & RAP Document Name \\
\hline $\mathrm{H}-3$ & Assessment of Tritium in the SRS Environment \\
\hline C-14 & Assessment of Radiocarbon in the SRS Environment \\
\hline P-32 & Assessment of Activation Products in the SRS Environment \\
\hline Ar-41 & Assessment of Noble Gases in the SRS Environment \\
\hline Cr-51 & Assessment of Activation Products in the SRS Environment \\
\hline Co-60 & Assessment of Activation Products in the SRS Environment \\
\hline $\mathrm{Zn}-65$ & Assessment of Activation Products in the SRS Environment \\
\hline $\mathrm{Kr}-85$ & Assessment of Noble Gases in the SRS Environment \\
\hline $\mathrm{Kr}-85 \mathrm{~m}$ & Assessment of Noble Gases in the SRS Environment \\
\hline Kr-87 & Assessment of Noble Gases in the SRS Environment \\
\hline $\mathrm{Kr}-88$ & Assessment of Noble Gases in the SRS Environment \\
\hline Sr-89 & Assessment of Strontium in the SRS Environment \\
\hline Sr-90 & Assessment of Strontium in the SRS Environment \\
\hline $\mathrm{Zr}-95$ & Assessment of Selected Fission Products in the SRS Environment \\
\hline $\mathrm{Nb}-95$ & Assessment of Selected Fission Products in the SRS Environment \\
\hline TC-99 & Assessment of Technetium in the SRS Environment \\
\hline Ru-103 & Assessment of Selected Fission Products in the SRS Environment \\
\hline Ru-106 & Assessment of Selected Fission Products in the SRS Environment \\
\hline $\mathrm{l}-129$ & Radioiodine in the SRS Environment \\
\hline $1-131$ & Radioiodine in the SRS Environment \\
\hline $\mathrm{Xe}-131 \mathrm{~m}$ & Assessment of Noble Gases in the SRS Environment \\
\hline Xe-133 & Assessment of Noble Gases in the SRS Environment \\
\hline $\mathrm{Xe}-135$ & Assessment of Noble Gases in the SRS Environment \\
\hline Cs-134 & Cesium in the SRS Environment \\
\hline Cs-135 & Cesium in the SRS Environment \\
\hline $\mathrm{Ce}-141$ & Assessment of Selected Fission Products in the SRS Environment \\
\hline $\mathrm{Ce}-144$ & Assessment of Selected Fission Products in the SRS Environment \\
\hline U-235 & Uranium in the SRS Environment \\
\hline $\mathrm{U}-238$ & Uranium in the SRS Environment \\
\hline Np-239 & Assessment of Neptunium, Americium, and Curium in the SRS Environment \\
\hline $\mathrm{Pu}-238$ & Assessment of Plutonium in the SRS Environment \\
\hline Pu-239 & Assessment of Plutonium in the SRS Environment \\
\hline $\mathrm{Am}-241$ & Assessment of Neptunium, Americium, and Curium in the SRS Environment \\
\hline $\mathrm{Cm}-244$ & Assessment of Neptunium, Americium, and Curium in the SRS Environment \\
\hline $\begin{array}{l}\text { Unidentified } \\
\text { Beta }\end{array}$ & Assessment of Strontium in the SRS Environment \\
\hline $\begin{array}{l}\text { Unidentified } \\
\text { Alpha }\end{array}$ & Assessment of Plutonium in the SRS Environment \\
\hline
\end{tabular}


In 1988, the U.S. Department of Energy's Savannah River Operations Office (DOE-SR), the Savannah River Site (SRS), and the Savannah River Ecology Laboratory (SREL) agreed to start a program to compile all knowledge of atmospheric and liquid releases of radioactive material to the environment both on and surrounding SRS. In addition, the radiation doses were to be calculated for the maximally exposed individual, the population within 50 miles of the Site, and the populations served by the water treatment plants using Savannah River water. The project was called the Radiological Assessment Program or RAP Program. After the program was under way, nonradioactive mercury was added to the study list. By 1998 , 13 documents had been written that described releases of the radionuclides in Table1-1 (see page 1-2).

More than 20 scientists from SRS and SREL participated in the program, contributing in their areas of expertise and historical knowledge (see Table 1-2).

\section{Table 1-2. Authors Contributing to RAP Docu- ments}

- L.R.Bauer

- W.H. Carlton

- B.R. del Carmen

- M. Denham

- L.L. Eldridge

- A.G.Evans

- L.A. Geary

- D.M. Hamby

- J.S. Haselow

- D.W. Hayes

- D.D. Hoel

- M.V. Kantelo

- E.J. Kvartek

- W.L. Marter

- H.L. Martin

- W.L. McDowell

- C.E. Murphy, Jr.

- M.C. Newman

- R.L. Nichols

- J.B. Pickett

- J.E. Pinder

- D.E. Stephenson

- R.N.Strom

- D.M. Tuck

- C.C. Zeigler
This current and last document in the series summarizes all the release data of the previous documents in which there is a significant radiation dose to the human population. In most instances, the dose reported in this summary document is different from the dose reported in the original documents. This is because of the changing technology in radiation dose assessment at SRS during the past decade. The ingestion parameters have been made more site-specific to reflect actual practices of the population surrounding SRS. Better meteorological data, measured with improved instrumentation located on the Site, have become available. The model for dose from liquid releases has been modified to better reflect the population dose from the consumption of salt water invertebrates.

Also, doses reported in the annual SRS environmental reports are different because different assumptions were used for this historic assessment.

No attempt has been made to reconstruct releases from original data; rather, summaries prepared over the years by qualified scientists and engineers have been accepted as accurate. Simplifying assumptions have been made for dose calculations. Atmospheric releases from F, H, C, K, $\mathrm{L}, \mathrm{P}$, and $\mathrm{R}$ Areas have been summed and assumed to originate from the center of the Site. Liquid and atmospheric releases were assumed to have occurred at a constant rate during the year. The offsite population was assumed to have remained fixed and equal to the 1980 census.

This summary, in conjunction with the 13 previously published RAP documents, contains the most comprehensive historical perspective of SRS releases and the best estimate of radiation dose to the offsite population. 
Assessment of Radionuclides

in the Savannah River Site Environment-Summary (U)

WSRC-TR-98-00162 


\section{Chapter 2. Origin and Disposition of Radionuclides at SRS}

This chapter presents an overview of the origin, uses, and disposition of radionuclides at SRS. The locations of Site facilities that had the potential to release radionuclides are shown in Figure 2.1. Radionuclide releases occurred in the reactor areas, $C, K, L, P$, and $R$; the separations areas, $F$ and $H$; the fuel fabrication area, $M$; and the Savannah River Technology Center (SRTC), A Area. The detailed history of releases from specific SRS facilities is presented in each of the documents listed at the end of this publication in "Additional Reading". 


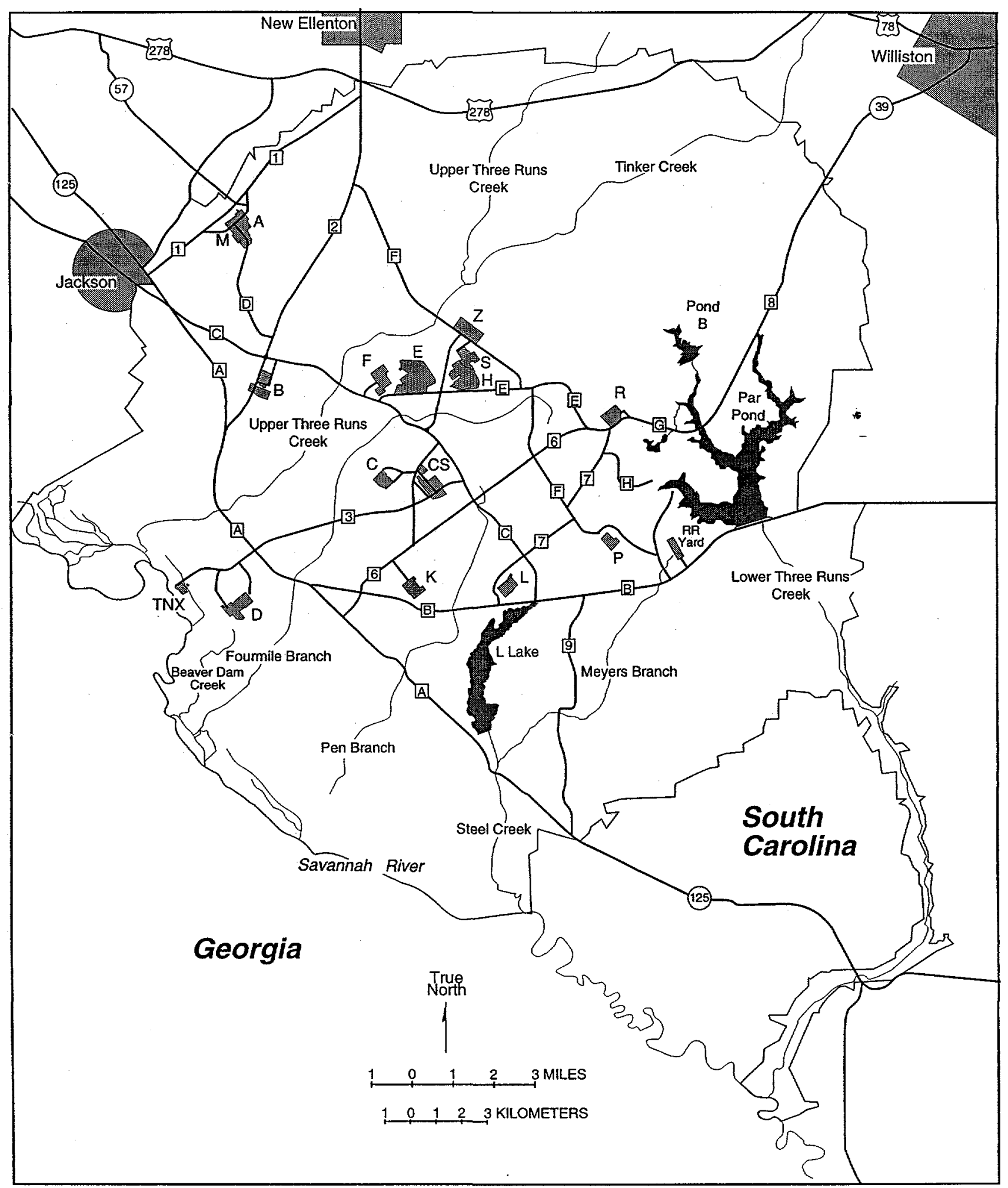

$93 \mathrm{~A} 030.03$

Figure 2.1. Areas Within SRS That May Be Sources of Radionuclide Releases 


\section{Irradiation in Production Reactors}

The role of the Site's five production reactors was to produce special nuclear materials-principally tritium and ${ }^{239} \mathrm{Pu}$-for national defense purposes. Additional radionuclides, such as ${ }^{238} \mathrm{Pu}$, which is a power source used for certain deep-space missions, and ${ }^{252} \mathrm{Cf}$, a neutron source used for medical and industrial applications, occasionally were produced for other government purposes.

The reactors became operational in 1953 through 1955 , but they did not operate continuously. They alternated between operating periods of production and periods for maintenance and fuel and target replacement. As of 1993, all five reactors were placed in cold shutdown.

The principal mechanism for ${ }^{239} \mathrm{Pu}$ production in the reactors was neutron capture by ${ }^{238} \mathrm{U}$. When a reactor was operating, neutron-induced fission reactions occurred in the ${ }^{235} \mathrm{U}$ fuel of the reactor core. In addition to fission products, each neutron-induced fission reaction in the fuel produced several neutrons, some of which induced additional fission reactions and maintained the chain reaction. Some of the remaining neutrons interacted with target materials in the reactor. The principal isotope resulting from neutron capture by ${ }^{238} \mathrm{U}$ was ${ }^{239} \mathrm{Pu}$. Following neutron capture by ${ }^{238} \mathrm{U}$, the ${ }^{239} \mathrm{U}$ rapidly decayed (with a 23.5-minute half-life) to ${ }^{239} \mathrm{~Np}$, which in turn decayed (with a 2.4-day half-life) to ${ }^{239} \mathrm{Pu}$.

Most of the radioactive material associated with SRS reactors was created when atoms fissioned. Many types of fission products were formed during the fissioning process, and several of them were released to the environment in significant quantities $\left({ }^{90} \mathrm{Sr}\right.$ and $\left.{ }^{137} \mathrm{Cs}\right)$.

Another process that created radioactive material was activation, in which a nucleus captured a fission neutron. In addition to neutron captures in the plutonium production process noted above, there also were neutron captures that created radioactive material which may or may not be useful. Some examples are ${ }^{60} \mathrm{Co}$ and ${ }^{41} \mathrm{Ar}$, which have been released in significant quantities during SRS operations.

\section{Radionuclides in Fuel and Targets Irradiated in Production Reactors}

Under ideal operating conditions, radionuclides were contained within the cladding of fuel and target elements during both irradiation and cooling. Cooling was the interval between the end of irradiation and the beginning of chemical separations. Irradiated materials were stored underwater in reactor basins. Beginning in the 1970s, the cooling time was at least 200 days for most irradiated materials.

Under normal operating conditions, it was possible for traces of radionuclides to escape from irradiated fuel and target elements to the reactor moderator or to the water in the cooling basins through small defects in the cladding. Air and water at the reactors were monitored for such releases. Actual releases are described in detail in Chapter 4.

Occasionally, the small defects developed into holes or splits in the cladding. This was called failure of the element. When a failure occurred, the reactor was shut down, and the failed element was transferred into a container called a "harp," which was stored underwater in the reactor basin and vented to the reactor stack. Failures occurred more frequently in the early years of operation than in later years. A failed element had the potential to contaminate the heavy water moderator with various radionuclides.

Water in the reactor cooling basins also became contaminated. Beginning in the $1960 \mathrm{~s}$, basin water was decontaminated routinely by passing the water through ion exchange resins to remove most of the radionuclides. Spent resins were reworked in the Resin Regeneration Facility in H Area or buried in the Burial Ground, which was renamed the Solid Waste Disposal Facility (SWDF) in 1990 .

After the cooling period, fuel and targets were treated in the chemical separations areas. During the chemical separations process, targets were treated by the Purex process in $\mathrm{F}$ Area to recover ${ }^{239} \mathrm{Pu},{ }^{237} \mathrm{~Np}$, and ${ }^{238} \mathrm{U}$ from irradiated ${ }^{238} \mathrm{U}$. The Purex process extracted plutonium and uranium into an organic solvent for separation and purification from waste products. Fuel was treated by the 
$\mathrm{HM}$ process in $\mathrm{H}$ Area to recover ${ }^{235} \mathrm{U}$ and ${ }^{237} \mathrm{~Np}$ from irradiated ${ }^{235} \mathrm{U}$; until 1959 , the Purex process was used in H Area. The principal difference was that the HM process used mercuric nitrate as a catalyst to enhance dissolution of the irradiated fuel. Also in $\mathrm{H}$ Area, ${ }^{238} \mathrm{Pu}$ occasionally was recovered from ${ }^{237} \mathrm{~Np}$ targets by the Frames process.

The clarification of dissolver solution and the solvent extraction of uranium and plutonium occurred in process tanks. The vapor space was exhausted to the process vessel vent system and then to the atmosphere through a sizegraded sand filter and 61-meter stacks.

Aqueous wastes from the chemical separations processes were evaporated and sent to underground storage tanks for radioactive waste. Condensate from the evaporation of stored liquid waste was sent to the separations area seepage basins until November 1988, when use of the seepage basins was terminated. Beginning in November 1988, condensate was sent to the Effluent Treatment Facility (ETF), where it was treated to remove radionuclides and chemicals before being discharged to Upper Three Runs Creek.

High-level liquid wastes generated in SRTC operations were stored in temporary waste tanks, where short-lived radionuclides decayed to insignificant levels. Periodically, the waste tanks' contents were shipped to F Area and processed through the waste system. Solid wastes generated in the SRTC handling operations were buried in the SWDF.

Most of the atmospheric and aqueous effluents in the chemical separations areas and SRTC have been monitored for possible releases. Measured releases are described in Chapter 3.

\section{Other Sources Due to SRS Operations}

Minute quantities of radionuclides were produced at SRS by test reactors and neutron activation analysis. The activity levels of these sources were insignificant when compared to activity levels in irradiated nuclear fuel and targets. However, these sources are discussed in the following subsections to provide a complete overview of potential releases.

\section{SRS Test Reactors}

Several small nuclear reactors were in use at two SRS locations from the 1950s through the 1970s. The Heavy Water Components Test Reactor (HWCTR), located in B Area, was used in the early 1960s to test prototype fuels for a proposed heavy water power reactor. The other test reactors were located in M Area. The Process Development Pile and the Lattice Test Reactor were used as zeropower mock-up facilities to test components for the production reactors. The Subcritical Experimental Pile also was used to test component designs. The Standard Pile provided neutrons for experiments such as neutron radiography and neutron activation.

\section{Neutron Activation Analysis}

Neutron activation analysis is an analytical technique for measurement of elemental compositions in materials. The ${ }^{252} \mathrm{Cf}$ neutron activation facilities and the $\mathrm{C}$-Area and $\mathrm{K}$ Area production reactors were used for the analysis of low levels of ${ }^{129} \mathrm{I}$ and uranium. For example, from the late 1970 s to the mid 1980s, environmental samples were activated in $\mathrm{C}$ Reactor to determine uranium content.

\section{Materials Originating Offsite}

Certain fuel irradiated at offsite noncommercial facilities was shipped to SRS for reprocessing. While awaiting reprocessing, the fuel was stored in the Receiving Basin for Offsite Fuel (RBOF), located in H Area. This fuel contained varying amounts of radionuclides, depending on the irradiation history.

Additional fission products were purchased from commercial vendors. The radionuclides were used at SRS and SREL for experimental purposes, such as chemical yield determination, and instrument calibration. The amounts were insignificant when compared to those produced in fuel and targets in SRS production reactors or in offsite fuel. 


\section{Chapter 3. Methods of Dose Calculation}

This chapter provides a technical description of the assumptions, models, and techniques used to calculate the radiation dose to offsite residents from the release of radioactive materials to the atmosphere or surface water.

In 1988, DOE issued internal dose conversion factors to ensure that doses are calculated in a consistent manner at all DOE facilities (DOE 1988). The factors, based on International Commission on Radiological Protection (ICRP) recommendations (ICRP 1979a), are used in conjunction with the models described later in this chapter to calculate all the doses reported in this document. 
This page intentionally left blank. 


\section{Models of Radioactive Material Transport and Dose}

Except for tritium, most of the radioactive materials released from SRS have such low concentrations in the offsite environment that they are not detectable by conventional monitoring techniques. Therefore, radiation doses to offsite individuals and populations are calculated with mathematical models. These models use known transport mechanisms for atmospheric and liquid releases and known major pathways of exposure to man. Modeled atmospheric and aqueous dispersion are verified periodically using environmental tritium measurements; tritium is released during normal SRS operations.

The first models used at SRS to calculate offsite doses were developed by SRTC (Cooper 1975). These models, MREM (atmospheric releases) and RIVDOSE (liquid releases), were used initially in 1972. MREM and RIVDOSE were replaced in 1982 with the more technologically advanced models now in use.

SRS annual offsite doses are currently calculated with the transport and dose models developed for the commercial nuclear industry (NRC 1977a, NRC 1977b). The models are implemented at SRS in the following computer programs:

\section{Atmospheric Releases}

- MAXIGASP calculates maximum and average doses to offsite individuals.

- POPGASP calculates offsite population collective dose.

\section{Liquid Releases}

- LADTAP II calculates both maximum and average doses to offsite individuals and collective dose to the offsite population.

MAXIGASP and POPGASP are SRTC-modified versions of the Nuclear Regulatory Commission (NRC) programs XOQDOQ (Sagendorf et al. 1982) and GASPAR (Eckerman et al. 1980). The modifications were made to meet the requirements for input of physical and biological data specific to SRS (Hamby 1991). The basic calculations in the XOQDOQ and GASPAR programs have not been modified. LADTAP II (Simpson and McGill $1980)$ is an essentially unaltered version of the NRC code of the same name. LADTAP XL, a spreadsheet version of LADTAP II, was used for dose modeling in this document.

\section{Modeling Atmospheric Dispersion of Radioac- tive Releases}

The routine atmospheric transport of radioactive materials from SRS is evaluated on the basis of meteorological conditions measured continuously at seven onsite towers. The towers relay wind speed, wind direction, and atmospheric stability information at 1.5-second intervals to SRTC via the WIND (Weather INformation and Display) system. A database of this information containing the 60-minute gverage values for the period 1987-1991 is accessed by the dispersion code to estimate downwind concentrations of released radionuclides.

Historically, offsite doses have been calculated using $\mathrm{H}$-Area meteorology and assuming that most releases occurred at the geographic center of the Site. It has been demonstrated that using data from one of the other onsite meteorological towers has little effect on the maximum individual dose and no effect on the 80 km population dose (Hamby and Parker 1991).

The dispersion of an atmospheric release from SRS is modeled using XOQDOQ, which computes concentrations in the plume as a function of downwind distance and compass sector. The plume is depleted because of dry deposition and radioactive decay. At the user's option, plume concentrations can be reduced by taking into account the effluent's upward displacement, which results from thermal buoyancy and/or momentum effects. This option is not used at SRS (i.e., no credit is taken for plume rise).

The plume concentration information generated by XOQDOQ then is used by the dose modeling program GASPAR to estimate doses to offsite individuals and populations. GASPAR estimates doses from a number of pathways, which are illustrated in a general sense in Figure 3.1. 


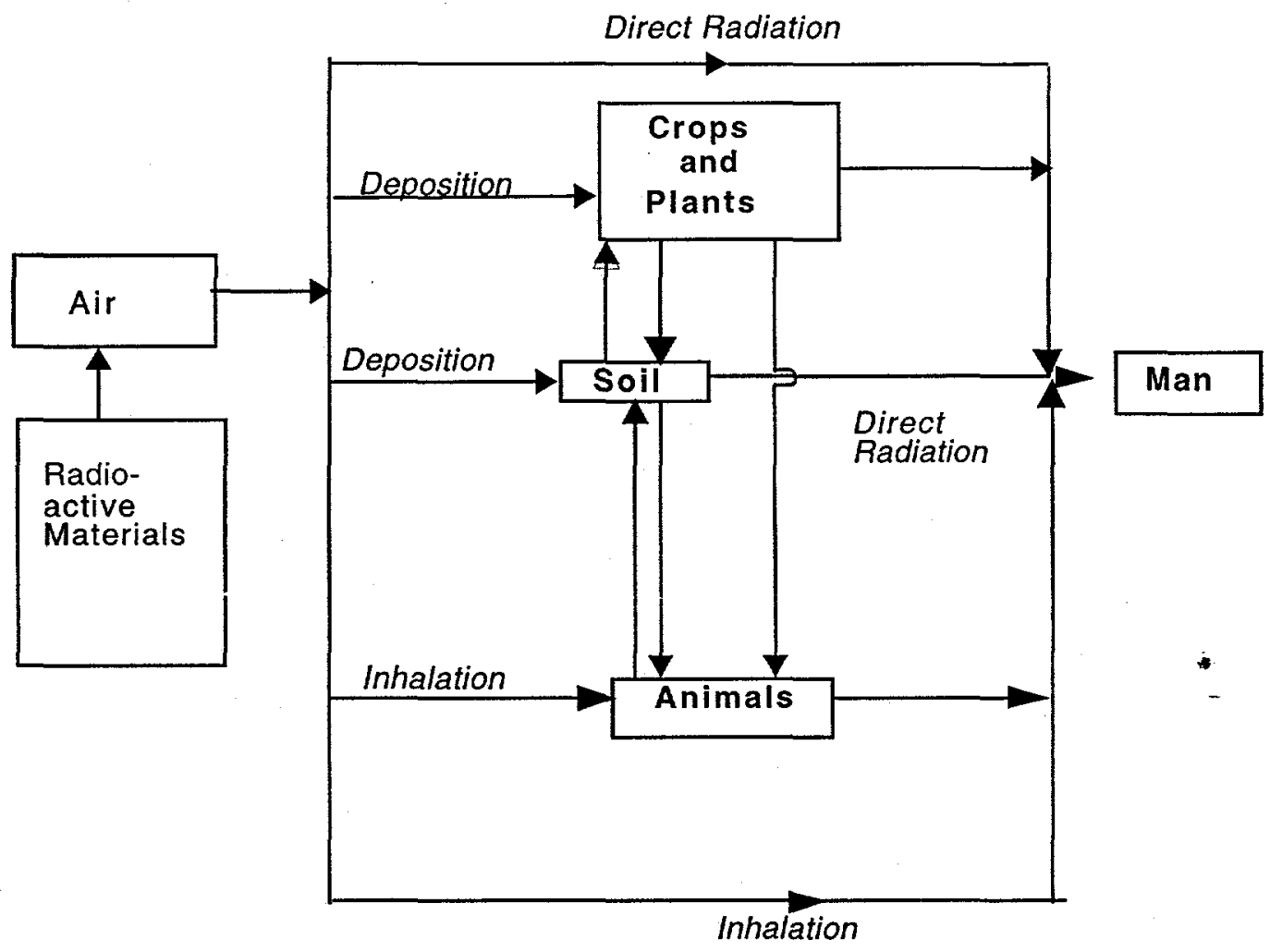

Figure 3.1. Simplified Pathways between Radioactive Materials Released to the Atmosphere and Man

The doses estimated by GASPAR are reported on a pathway-specific basis, as follows:

- Plume-external dose from radioactive materials suspended in the atmosphere

- Ground-external dose from radioactive materials deposited on the ground

- Inhalation-internal dose from inhalation of radioactive materials present in the plume

- Vegetation-internal dose from consumption of contaminated crops

- Milk-internal dose from milk produced in a contaminated area

- Meat-internal dose from consumption of meat produced in a contaminated area

The codes XOQDOQ (Bauer 1991), GASPAR (Hamby 1992), and MAXIGASP and POPGASP (Hamby 1995) have undergone comprehensive reviews in association with the Westinghouse Savannah River Company (WSRC) quality assurance requirements for software.

\section{MAXIGASP}

The calculations required by XOQDOQ and GASPAR to estimate maximum and average individual doses are performed at SRS using MAXIGASP. MAXIGASP calculates annual average ground-level air concentrations and 50 -year committed doses at a number of points along the Site boundary in each of 16 compass sectors.

The main outputs from the program are the maximum potential effective dose equivalents to an individual along the SRS perimeter. The maximally exposed individual is assumed to reside continuously at the location of highest exposure and to have living and eating habits that maximize dose. These assumptions provide a ceiling on doses from atmospheric releases, because no such individual is believed to exist. The parameters used to calculate doses with MAXIGASP are presented in Table 3-1.

\section{POPGASP}

The calculations required by XOQDOQ and GASPAR to estimate population doses from atmospheric releases are performed at SRS using POPGASP. POPGASP calculates 
annual average ground-level air concentrations and annual doses for each of 160 regions ( 16 wind direction sectors at 10 distances per sector) within an $80-\mathrm{km}$ radius of the release location.

In addition to compass sector-specific meteorological information, POPGASP uses sector-specific data on population distribution and composition. Comparable data on milk, meat, and vegetable production/consumption also are used in the code. These databases are extensive and are available for review in the SRS annual environmental reports. With respect to the human parameters used in POPGASP, the key values are shown in Table 3-1.

Table 3-1. Site-Specific Parameters for Atmospheric Releases

\begin{tabular}{|c|c|}
\hline Population Group & \\
\hline $80-\mathrm{km}$ radius & $\begin{array}{c}555,100 \\
\text { (1980 Census) }\end{array}$ \\
\hline $\begin{array}{c}\text { Maximum Individual } \\
\text { (MAXIGASP) } \\
\text { Exposure Pathway }\end{array}$ & \\
\hline Inhalation $\left(\mathrm{m}^{3} / \mathrm{yr}\right)$ & 8,000 \\
\hline Ingestion & \\
\hline Cow's milk (L/yr) & 230 \\
\hline Meat $(\mathrm{kg} / \mathrm{yr})$ & 81 \\
\hline $\begin{array}{l}\text { Leafy vegetables } \\
\text { (kg/yr) }\end{array}$ & 43 \\
\hline $\begin{array}{c}\text { Fruits, grains, and } \\
\text { other vegetables } \\
(\mathrm{kg} / \mathrm{yr})\end{array}$ & 276 \\
\hline External exposure & \\
\hline $\begin{array}{l}\text { Transmission factor } \\
\text { for shielding from } \\
\text { buildings }\end{array}$ & 0.7 \\
\hline $\begin{array}{c}\text { General Population } \\
\text { (POPGASP) } \\
\text { Exposure Pathway }\end{array}$ & \\
\hline Inhalation ( $\left.\mathrm{m}^{3} / \mathrm{yr}\right)$ & 8,000 \\
\hline Ingestion & \\
\hline Cow's milk (L/yr) & 120 \\
\hline Meat (kg/yr) & 43 \\
\hline $\begin{array}{l}\text { Leafy vegetables } \\
(\mathrm{kg} / \mathrm{yr})\end{array}$ & 21 \\
\hline $\begin{array}{c}\text { Fruits, grains, and } \\
\text { other vegetables } \\
(\mathrm{kg} / \mathrm{yr})\end{array}$ & 163 \\
\hline External exposure & \\
\hline $\begin{array}{l}\text { Transmission factor } \\
\text { for shielding from } \\
\text { buildings }\end{array}$ & 0.5 \\
\hline
\end{tabular}

\section{Modeling Doses from Liquid Releases}

The consequences of liquid releases from SRS are modeled using LADTAP XL, a spreadsheet version of LADTAP II (Liquid Annual Doses To All Persons). The potential pathways of exposure from liquid releases to the environment are shown in Figure 3.2. The pathway-specific doses calculated by LADTAP are grouped into the following categories:

- Potable drinking water-internal dose from consuming drinking water of Savannah River origin

- Sport fish and commercial fish-internal dose from consuming fish of Savannah River origin

- Salt water invertebrates-internal dose from consuming shellfish from the estuary of the Savannah River

- Recreation-external dose from recreation activities (boating, swimming, and shoreline) in and along the Savannah River

- Irrigation-internal dose from foods produced with Savannah River water irrigation (there are no known users of the river for this purpose)

LADTAP XL estimates individual and population doses at specific downstream locations. The only removal mechanism included in the transport model as it is used at SRS is radioactive decay. With the exception of cesium, no credit is taken for adsorption on stream sediments.

One major difference between LADTAP II and LADTAP $\mathrm{XL}$ is the method of calculating population dose. LADTAP II uses only a fraction of the dose from fish caught in the Savannah River. The fraction is the ratio of the $80-\mathrm{km}$ harvest to the U.S. harvest. LADTAP XI assigns the dose from all sport and commercial fish caught in the Savannah River to the population dose. LADTAP XL, used for dose calculations in this document, is conservative and reports the maximum possible population dose.

One major assumption inherent in the application of LADTAP XL to SRS releases is that liquid discharges undergo complete mixing in the Savannah River before reaching potentially exposed populations. This assumption is supported by repeated measurements indicating that complete mixing occurs in the river between SRS and the Highway

LADTAP XL generates maximum individual and population doses for all the exposure pathways identified above. Though standard input values were provided in LADTAP II, SRS calculations are performed with site-specific information. Principal input values used in the SRS version of LADTAP XL are shown in Table 3-2. Population dose parameters are shown in Table 3-3. 


\section{Table 3-2. Site-Specific Parameters for Liquid Releases}

\begin{tabular}{|c|c|}
\hline $\begin{array}{l}\text { Maximally Exposed } \\
\text { Individual Dose Assessments } \\
\text { (LADTAP XL) }\end{array}$ & \\
\hline \multicolumn{2}{|l|}{ Site Parameters } \\
\hline $\begin{array}{l}\text { Savannah River flow rate } \\
\left(\mathrm{m}^{3} / \mathrm{sec}\right)\end{array}$ & $\begin{array}{l}\text { Measured annual } \\
\text { average }\end{array}$ \\
\hline $\begin{array}{l}\text { Transit time from } \\
\text { SRS to Savannah River }(\mathrm{hr})\end{array}$ & 24 \\
\hline Shore-width factor & 0.2 \\
\hline \multicolumn{2}{|l|}{ Human Parameters } \\
\hline Water consumption (L/yr) & 730 \\
\hline Fish consumption (kg/yr) & 19 \\
\hline Shellfish consumption (kg/yr) & 8 \\
\hline Shoreline recreation (hr/yr) & 23 \\
\hline Swimming (hr/yr) & 8.9 \\
\hline Boating (hr/yr) & 21 \\
\hline
\end{tabular}

Radionuclide concentrations in the Savannah River are diluted by the inflow of streams downriver of SRS. Additional dilution occurs at the Beaufort-Jasper, South Carolina water treatment plant from the inflow of surface water and at the Port Wentworth, Georgia water treatment plant because of the close proximity of Abercorn Creek to the intake. Tritium is readily measured in the processed water of each system (Hayes and Marter 1991). Calculation of dose for the water treatment plants-based on a "derived" river flow rate-takes into account dilution of the river water.

\section{Validation of Transport Models Using Monitoring Data}

\section{Atmospheric Releases}

The radionuclide concentrations predicted by XOQDOQ are compared annually with measured values of tritium concentrations in air to evaluate the performance of the code. Tritium is the only radionuclide released by SRS that can be detected routinely offsite with conventional measuring techniques. Predicted values tend to exceed observed values, but not to a degree that would indicate an excessively conservative approach 301 sampling station (Arnett et al. 1994).
Table 3-3. Additional Site-Specific Parameters for Liquid Releases

\begin{tabular}{|c|c|}
\hline \multicolumn{2}{|c|}{$\begin{array}{l}\text { Population Dose Assessments } \\
\text { (LADTAP XL) }\end{array}$} \\
\hline Site Parameters & \\
\hline $\begin{array}{l}\text { Savannah River flow rate } \\
\qquad\left(\mathrm{m}^{3} / \mathrm{sec}\right)\end{array}$ & $\begin{array}{c}\text { Measured } \\
\text { annual average }\end{array}$ \\
\hline $\begin{array}{l}\text { Transit time from SRS } \\
\text { to Savannah River (hr) }\end{array}$ & 24 \\
\hline $\begin{array}{l}\text { Transit time from SRS } \\
\text { to water treatment plants }(\mathrm{hr})\end{array}$ & 72 \\
\hline $\begin{array}{l}\text { Retention time in } \\
\text { water treatment system (hr) }\end{array}$ & 24 \\
\hline Shore-width factor & 0.2 \\
\hline River dilution in estuary & 3 \\
\hline $\begin{array}{c}\text { Aquatic food havest, edible portions } \\
(\mathrm{kg} / \mathrm{yr})\end{array}$ & \\
\hline Sport fish & $\div 35,000$ \\
\hline Commercial fish & $-2,700$ \\
\hline Saltwater invertebrates & 390,000 \\
\hline Human Parameters & \\
\hline Water consumption (L/yr) & 370 \\
\hline Fish consumption $(\mathrm{kg} / \mathrm{yr})$ & 9 \\
\hline Shellfish consumption (kg/yr) & 2 \\
\hline Shoreline recreation (hr/yr) & 960,000 \\
\hline Swimming (hr/yr) & 160,000 \\
\hline Boating (hr/yr) & $1,100,000$ \\
\hline
\end{tabular}

Other comparisons of predicted and measured concentrations have been made (Simpkins 1995) and have exhibited similar results. The available data suggest that calculated concentrations of tritium in air generally are conservative estimates of actual offsite values.

\section{Liquid Releases}

The Savannah River flow rate is one of the major parameters in the dose calculations. The flow rate is taken from U.S. Geological Survey (USGS) records and can be verified by comparison with concentrations of tritium in river samples. 


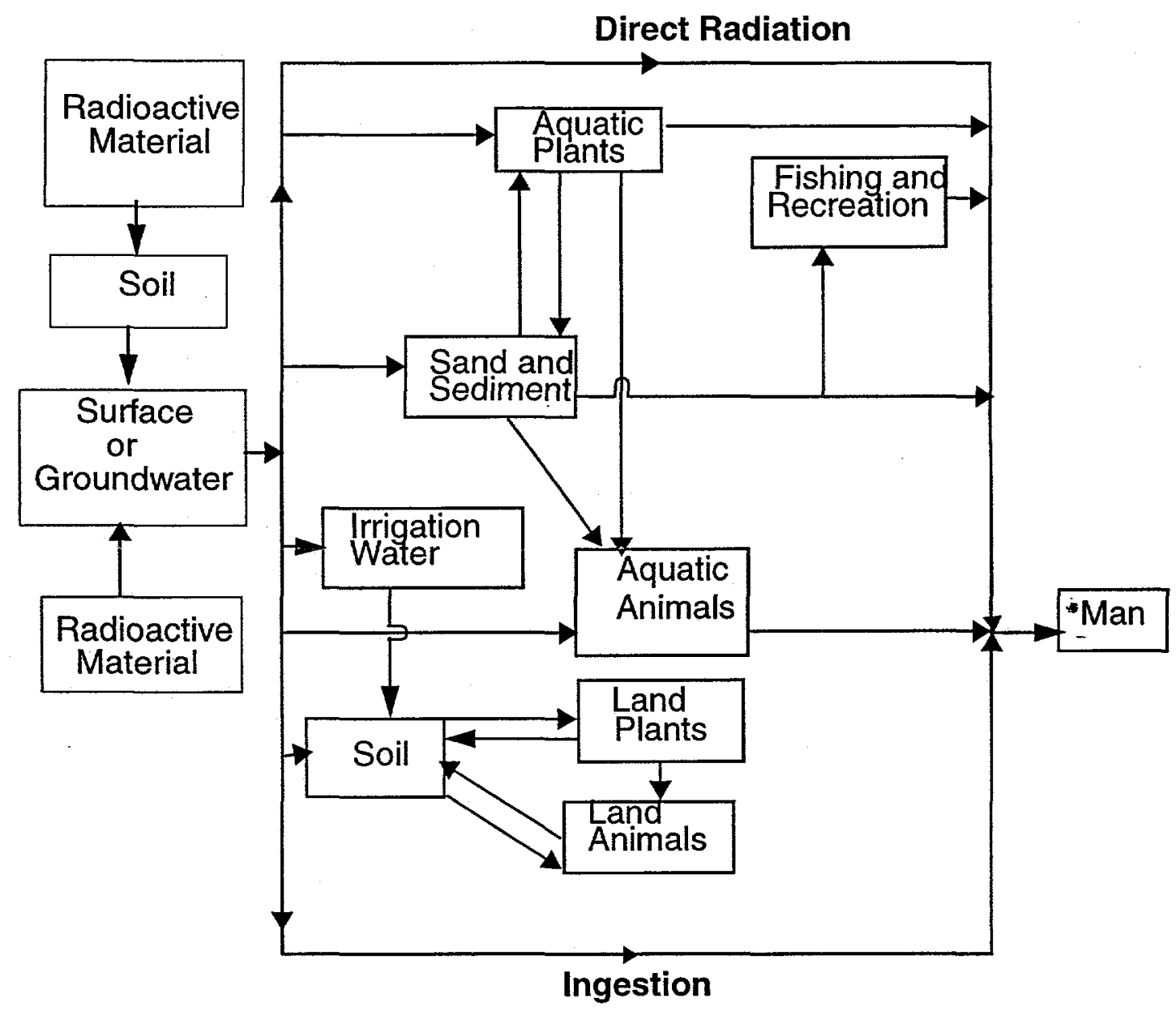

Figure 3.2. Simplified Pathways between Radioactive Materials Released to Groundwater or Surface Waters and Man

\section{References for Chapter 3}

Arnett, M.W., L.K. Karapatakis, and A.R. Mamatey, 1994, Savannah River Site Environmental Report for 1993 (WSRC-TR-94-075), Westinghouse Savannah River Company, Aiken, South Carolina.

Bauer, L.R., 1991, Modeling Chronic Atmospheric Releases at the SRS: Evaluation and Verification of XOQDOQ (U) (WSRC-RP-91-320), Westinghouse Savannah River Company, Aiken, South Carolina.

Cooper, R.E., 1975, Computer Programs at SRL to Evaluate Environmental Effects of SRP Operations and Postulated Accidental Releases (DPST75-384), Savannah River Laboratory, Aiken, South Carolina.
U.S. Department of Energy, 1988, Internal Dose Conversion Factors for Calculation of Dose to the Public, DOE/EH-0071, Washington, DC.

Eckerman, K.F., F.J. Congel, A.K. Roecklein, and W.J. Pasciak, 1980, User's Guide to GASPAR Code, NUREG-0597, U.S. Nuclear Regulatory Commission, Washington, DC.

Hamby, D.M., 1991, Land and Water Use Characteristics in the Vicinity of the Savannah River Site $(U)$ (WSRC-RP-91-17), Westinghouse Savannah River Company, Aiken, South Carolina.

Hamby, D.M., and M.J. Parker, 1991, Gaussian Dispersion and Dosimetric Modeling Sensitivity to Area-specific1982-1986 Meteorological Data Collected at the Savannah River Site $(U)$ 
(WSRC-RP-91-909), Westinghouse Savannah River Company, South Carolina.

Hamby, D.M., 1992, Verification of the GASPAR Dose Assessment Module Used in MAXIGASP and POPGASP (WSRC-RP-92-418), Westinghouse Savannah River Company. Aiken, South Carolina.

Hamby, D.M., 1995, Verification of the MAXIGASP and POPGASP Computer Codes for Environmental Dose Assessment (WSRC-RP-94-522), Westinghouse Savannah River Company, Aiken, South Carolina.

Hayes, D.W., and W.L. Marter, 1991, Historical River Flow Rates for Dose Calculations (SRL-ETS910257), Westinghouse Savannah River Company. Aiken, South Carolina

International Commission on Radiological Protection, 1979a, Limits for Intake of Radionuclides by Workers, Oxford: Pergamon Press; ICRP Publication 30, Part 1.

U.S. Nuclear Regulatory Commission, 1977a, Methods for Estimating Atmospheric Transport and Dispersion of Gaseous Effluents in Routine Releases from LightWater-Cooled Reactors, Regulatory Guide 1.111, Rev. 1, Washington, DC.
U.S. Nuclear Regulatory Commission, 1977b, Calculation of Annual Doses to Man from Routine Releases of Reactor Effluents for the Purpose of Evaluating Compliance with 10 CFR Part 50, Appendix I, Regulatory Guide 1.109, Rev. 1, Washington, DC.

Sagendorf, J.F., J.T. Goll, and W.F. Sandusky, 1982, XOQDOQ: Computer Program for the Meteorological Evaluation of Routine Effluent Releases at Nuclear Power Stations, NUREG/CR-2919, U. S. Regulatory Commission, Washington, D.C.

Simpkins, A.A., and D. M. Hamby, 1997, "Predicted versus Measured Tritium Oxide Concentration at the Savannah River Plant", Health Physics 72(2):179185.

Simpson, D.B., and B.L. McGill, 1980, Users Manual for LADTAP II-A Computer Program for Calculating Radiation Exposure to Man from Routine Releases of Nuclear Reactor Effluents, NUREG/CR-1276, ORNL/TDMC-1, Oak Ridge, Tennessee. 


\section{Chapter 4. Releases and Doses of Individual Radio- nulclides}

Doses are reported in this chapter for all radionuclides released to the atmosphere or surface water in sufficient quantity to result in a population dose of 1 person-rem. Releases from SRS facilities have been reported in various Site documents (Cummins et al. 1991a; Cummins et al. 1991b; Arnett et al. 1992; Arnett et al. 1993; Arnett et al. 1994; Arnett et al. 1995; Arnett et al. 1996; Arnett et al. 1997). 
Assessment of Radionuclides

in the Savannah River Site Environment-Summary (U)

WSRC-TR-98-00162

This page intentionally left blank 


\section{Activation Products}

\section{Phosphorus-32}

During normal reactor operations at SRS, small amounts of ${ }^{32} \mathrm{P}$ were in the moderator; these originated from $n, p$ activation of sulfur leached from moderator deionizers (Longtin 1966). In the mid-1960s, phosphoric acid, $\mathrm{H}_{3} \mathrm{PO}_{4}$, was used to clean heat exchangers, and the residual ${ }^{31} \mathrm{P}$ was converted to radioactive ${ }^{32} \mathrm{P}$ by neutron absorption (Ashley 1966). When reactor elements were discharged to the disassembly basin, ${ }^{32} \mathrm{P}$ on the outside surfaces leached into disassembly basin water. Continuous purging of the basin water was the primary pathway by which aqueous activation products were released to the environment. The basin water initially was purged directly to Site streams to remove the heat generated by the stored irradiated fuel and targets and to maintain clarity in the storage basins. After installation of basin heat exchangers, deionizers, and filters in the 1960s, the volume of purged water decreased significantly, as did the release of radioactivity.

The release of ${ }^{32} \mathrm{P}$ to Site streams resulted in a dose of $46 \mathrm{mrem}$ to the maximally exposed individual, with drinking water doses well below $1 \mathrm{mrem}$. The population dose was 110 person-rem, or $2.3 \%$ of the total population dose from SRS operations. Most of the dose was a result of eating fish and saltwater invertebrates that had accumulated the 32P. Two-thirds of the dose occurred in 1965 and 1966 (see Table 4-1).

\section{Chromium-51}

Chromium-51 activity in the moderator originated from activation of stable ${ }^{50} \mathrm{Cr}$ in stainless steel reactor components in the reactor tank. Additional ${ }^{51} \mathrm{Cr}$ was produced from ${ }^{50} \mathrm{Cr}$ contained in erosion and corrosion products of stainless steel used in the reactor cooling system piping (Longtin 1972). The ${ }^{51} \mathrm{Cr}$ was formed when the erosion and corrosion products were transported into the reactor vessel and exposed to neutrons. Chromium-51 was released to Site streams in a manner identical to that of ${ }^{32} \mathrm{P}$.
The release of ${ }^{51} \mathrm{Cr}$ to Site streams resulted in a dose of 0.22 mrem to the maximally exposed individual; drinking water doses at the water treatment plants were less than $0.03 \mathrm{mrem}$. The population dose, 9.6 person-rem, resulted primarily from consumption of fish and saltwater invertebrates and occurred primarily in the mid 1960 s (see Table $4-2$ ). Overall, ${ }^{51} \mathrm{Cr}$ releases accounted for $0.2 \%$ of the population dose from SRS operations.

\section{Cobalt-60}

Most atmospheric ${ }^{60} \mathrm{Co}$ releases came from SRTC during the period 1968-1984. The releases were the result of research on a thermoelectric generator program that used many thousands of curies of ${ }^{60} \mathrm{Co}$ as the heat source (Angerman 1973; Zecha 1987).

The dose to the maximally exposed individual was 0.76 mrem. Most of this dose came from SRTC releases because that facility was much closer to the Site boundary than the reactor and separation facilities (see Table 4-3). The population dose, 0.65 personrem, was less dependent on the proximity of SRTC to the Site boundary.

Cobalt-60 activity in the moderator originated in a manner similar to ${ }^{51} \mathrm{Cr}$ activity through the activation of ${ }^{59} \mathrm{Co}$ contained in erosion and corrosion products. In addition, ${ }^{60} \mathrm{Co}$ was produced for gamma radiation and heat sources (Bebbington 1990).

Cobalt-60 was released to Site streams in a manner identical to that of ${ }^{32} \mathrm{P}$. The dose from liquid releases of ${ }^{60} \mathrm{Co}$ was $0.44 \mathrm{mrem}$ to the maximally exposed individual, and drinking water doses at the water treatment plants were less than $0.1 \mathrm{mrem}$. The population dose, 28 person-rem, resulted primarily from eating fish and saltwater invertebrates and occurred primarily in the 1960s (see Table 4-4). Overall, ${ }^{60} \mathrm{Co}$ releases accounted for $0.6 \%$ of the population dose from SRS operations.

\section{Zinc-65}

Zinc-65 activity in the moderator originated from neutron activation of stable ${ }^{64} \mathrm{Zn}$, which was found as a trace element in aluminum reactor fuel and target components (Fox 1975). Zinc-65 was released to Site 
streams in a manner identical to that of ${ }^{32} \mathrm{P}$. The dose to the maximally exposed individual was $7.3 \mathrm{mrem}$, and the drinking water dose at the water treatment plants was 0.11 mrem or less. The population dose, 1,100 person-rem, resulted primarily from eating fish and saltwater invertebrates and occurred primarily in the early 1960s (see Table 4-5). Overall, ${ }^{65} \mathrm{Zn}$ releases accounted for $23 \%$ of the population dose from SRS operations.

Zinc-65 accounted for more than two-thirds of the total population dose from liquid releases of all radionuclides. Most of this dose resulted from eating saltwater invertebrates that had bioaccumulated $65 \mathrm{Zn}$. The bioaccumulation factor for zinc is 2,000 for fish and 50,000 for saltwater invertebrates, which means that the concentration of zinc in the fisli tissue is 2,000 times as great as the concentration in river water.

\section{Carbon-14}

SRS produced ${ }^{14} \mathrm{C}$ by various reactions in the fuel, moderator, and core construction materials in SRS production reactors. The mechanisms included neutron-induced reactions $[(n, p) ;(n, \alpha) ;$ and $(n, \gamma)]$ and ternary fission (Hayes and MacMurdo 1977). The (n,p) reaction produced ${ }^{14} \mathrm{C}$ by reaction of neutrons with ${ }^{14} \mathrm{~N}$. Nitrogen occurred as an impurity in the fuel, as dissolved gas, as nitric acid, as ammonium hydroxide (used for $\mathrm{pH}$ control purposes in the moderator), and as an impurity in the core material. Small quantities of ${ }^{14} \mathrm{C}$ also were produced by the $(n, p)$ reaction with nitrogen in the air in the annular cavity outside the reactor tank. The $(n, \alpha)$ reaction occurred primarily with ${ }^{17} \mathrm{O}$ in the moderator. The $(\mathrm{n}, \gamma)$ reaction with ${ }^{13} \mathrm{C}$ produced a negligible amount of ${ }^{14} \mathrm{C}$ in SRS reactors, which released ${ }^{14} \mathrm{C}$ to the atmosphere as ${ }^{14} \mathrm{CO}$ and ${ }^{14} \mathrm{CO}_{2}$ through their ventilation systems.

Radiocarbon releases from the separations facilities were to the atmosphere. Dissolution of fuel and targets in strong nitric acid solutions assured the oxidation and volatilization of any carbon compounds in the fuel and target elements during processing. Atmospheric releases of ${ }^{14} \mathrm{C}$ were calculated from known operating power levels and fuel types. using the assumptions given in Hayes and MacMurdo (1977). In more recent years, stack releases of ${ }^{14} \mathrm{C}$ have been measured to confirm the calculated data.
Approximately $3.0 \times 10^{3} \mathrm{Ci}$ of ${ }^{14} \mathrm{C}$ was released to the atmosphere from 1954 through 1996. The dose to the maximally exposed individual at the Site boundary was about 1 mrem, and the population dose was 30 personrem, or $0.6 \%$ of the total population dose from SRS operations (see Table 4-6).

There have been no recorded liquid releases of ${ }^{14} \mathrm{C}$.

\section{Cesium-137}

The principal mechanism for production of ${ }^{137} \mathrm{Cs}$ was neutron-induced fission in the reactors. When a reactor was operating, neutron-induced fission reactions occurred in the $235 \mathrm{U}$ fuel of the reactor core. Fission reactions formed a variety of fission products, which included isotopes of cesium. Additional ${ }^{137} \mathrm{Cs}$ was formed in the reactor as a result of neutron activation of stable cesium generated by neutron fission.

There were no recorded atmospheric ${ }^{137} \mathrm{Cs}$ releases from the reactors. Most of the atmospheric ${ }^{137} \mathrm{Cs}$ released from the separation areas was the result of two incidents. The first occurred in 1955 during start-up, primarily as a result of leakage around the sand filter bypass plug. The second occurred in 1987, when an evaporator steam flange failed in the waste management facility.

Approximately $3.5 \mathrm{Ci}$ of ${ }^{137} \mathrm{Cs}$ were released to the atmosphere from 1955 through 1996 . The dose to the maximally exposed individual at the Site boundary was about $0.5 \mathrm{mrem}$, and the population dose was 34 person-rem or $0.7 \%$ of the total population dose from SRS operations (see Table 4-7).

Most of the liquid ${ }^{137} \mathrm{Cs}$ releases were from the reactors as a result of leaking fuel elements in the 1950s and 1960s. The fuel elements were stored in disassembly basins, and ${ }^{137} \mathrm{Cs}$ was released to Site streams when basin water was purged to maintain clarity and remove heat. More than $600 \mathrm{Ci}$ were released, but only about one-third reached the Savannah River. Approximately two-thirds remain in the stream beds, flood plains, ponds, and swamps on or near SRS.

The dose from liquid releases of ${ }^{137} \mathrm{Cs}$ was $61 \mathrm{mrem}$ to the maximally exposed individual and drinking water doses at the water treatment plants were less than 1 mrem. The 
population dose, 130 person-rem, resulted primarily from consumption of fish and saltwater invertebrates and occurred primarily in the 1960s (see Table 4-8). Overall, ${ }^{137} \mathrm{Cs}$ accounted for $3.3 \%$ of the population dose from Site operations.

\section{lodine-129 and lodine-131}

The principal mechanism for production of ${ }^{129} \mathrm{I}$ and ${ }^{131} \mathrm{I}$ was neutron-induced fission in the reactors. When a reactor was operating, neutron-induced fission reactions occurred in the ${ }^{235} \mathrm{U}$ fuel of the reactor core. Fission reactions formed a variety of fission products, which included several isotopes of iodine. The two largest contributors to environmental dose were ${ }^{129} \mathrm{I}$ and ${ }^{131} \mathrm{I}$.

Iodine was released to the atmosphere when the fuel and target elements were chemically dissolved in F Area and $\mathrm{H}$ Area. The quantity of ${ }^{131}$ I released depended on the cooling time between reactor shutdown and dissolution of the elements. Cooling times were much shorter during the $1950 \mathrm{~s}$, when there was a greater sense of production urgency.

Approximately $5.7 \mathrm{Ci}$ of ${ }^{129} \mathrm{I}$ were released to the atmosphere from 1955 through 1996. The dose to the maximally exposed individual at the Site boundary was 3.9 mrem, and the population dose was 100 person-rem, or $2.1 \%$ of the total population dose from SRS operations (see Table 4-9).

Approximately $2,500 \mathrm{Ci}$ of ${ }^{131}$ I were released to the atmosphere from 1955 through 1996. The dose to the maximally exposed individual at the Site boundary was 21 mrem and the population dose was 830 person-rem (see Table 4-9).

During normal reactor operations, small amounts of $131 \mathrm{I}$ were in the moderator; these originated from leaks in the fuel and target elements. When reactor elements were discharged to the disassembly basin, ${ }^{131}$ I on the outside surfaces leached into disassembly basin water. Continuous purge of the basin water was the primary pathway by which aqueous ${ }^{131}$ I was released to the environment. The basin waters initially were purged directly to Site streams to remove the heat generated by the stored irradiated fuel and targets and to maintain clarity in the storage basins. After installation of basin heat exchangers, deionizers, and filters in the 1960s, the volume of purged water decreased significantly, as did the release of radioactivity. Approximately $300 \mathrm{Ci}$ of ${ }^{131} \mathrm{I}$ were released to streams from 1954 through 1996.

The dose from liquid releases of ${ }^{131} \mathrm{I}$ was $1.5 \mathrm{mrem}$ to the maximally exposed individual, and drinking water doses at the water treatment plants were less than 1 mrem. The population dose was 11 person-rem (see Table 4-10). Overall, ${ }^{131}$ I releases accounted for $18 \%$ of the population dose from Site operations.

\section{Americium and Curium}

\section{Americium-241}

While ${ }^{239} \mathrm{Pu}$ was produced as a component of nuclear weapons, other isotopes of plutonium, especially $240 \mathrm{Pu}$ and ${ }^{241} \mathrm{Pu}$, were produced in SRS reactors, principally as unwanted byproducts resulting from neutron capture by ${ }^{239} \mathrm{Pu}$. The ${ }^{240} \mathrm{Pu}$ content was minimized intentionally because of its adverse effect on weapons yield. The ${ }^{241} \mathrm{Pu}$ content was kept low intentionally to minimize buildup of its daughter, ${ }^{241} \mathrm{Am}$. The decay daughters of ${ }^{241}$ Am emit gamma radiation, which increases personnel exposure during handling and storage of nuclear weapons.

Americium-241 was released to the atmosphere principally from F Area and $\mathrm{H}$ Area during radiochemical processing of irradiated fuel and targets. The total reported release was $6.1 \times 10^{-3} \mathrm{Ci}$ from 1977 through 1996 . Prior to 1977 , no specific analysis for ${ }^{241} \mathrm{Am}$ was conducted. The dose to the maximally exposed individual at the Site boundary was $0.02 \mathrm{mrem}$, and the population dose was 1.2 person-rem, or less than $0.1 \%$ of the total population dose from SRS operations (see Table 4-11).

\section{Curium-244}

Beginning in 1963, transplutonium isotopes were prepared by placing ${ }^{239} \mathrm{Pu}$ targets in high-flux charges in SRS reactors. After the targets were dissolved and processed in a separation facility, they were delivered to SRTC for further processing. The work involved gram quantities of curium and americium, microgram quantities of californium and berkelium, and nanogram quantities of einsteinium. By 1968, approximately $5 \mathrm{~kg}$ of ${ }^{244} \mathrm{Cm}$ had been recovered (Moyer 1968). 
Atmospheric ${ }^{244} \mathrm{Cm}$ releases were reported for F Area and $\mathrm{H}$ Area, but the majority of released material came from A Area during the years when research was conducted on the use of ${ }^{244} \mathrm{Cm}$ as a heat source for electricity generation (Stoddard 1964). A total of $9.0 \times 10^{-2} \mathrm{Ci}$ of ${ }^{244} \mathrm{Cm}$ was released to the atmosphere with $5.3 \times 10^{-2} \mathrm{Ci}$ from A Area.

The dose to the maximally exposed individual was 0.15 mrem. Most of this dose came from SRTC releases because that facility was much closer to the Site boundary than the reactor and separation facilities (see Table 4-12). The population dose, 8.9 person-rem, was less dependent on the proximity of SRTC to the Site boundary.

Liquid releases of $244 \mathrm{Cm}$ were reported only from A Area during the years when research was conducted on the use of ${ }^{244} \mathrm{Cm}$ as a heat source for electricity generation. The releases to Site streams resulted in a dose of $0.11 \mathrm{mrem}$ to the maximally exposed individual, with drinking water doses of 0.05 mrem or less. The population dose was 12 person-rem (see Table 4-13). The total population dose from releases of ${ }^{244} \mathrm{Cm}$ was 21 person-rem, about $0.4 \%$ of the dose from all SRS operations from 1954 through 1996.

\section{Noble Gases, Argon-41}

Argon-41 originated at SRS as an activation product when neutrons produced in SRS's reactor vessels irradiated air surrounding the vessel. Stable ${ }^{40} \mathrm{Ar}$ captured a neutron and became ${ }^{41} \mathrm{Ar}$, which was swept from the vicinity of the reactor vessel and exhausted through a 61-meter stack.

A total of $6.4 \times 10^{6} \mathrm{Ci}$ of ${ }^{41} \mathrm{Ar}$ were released to the atmosphere from the five production reactors. The dose to the maximally exposed individual was $7.8 \mathrm{mrem}$, and the population dose was 190 person-rem, or about $4 \%$ of the total population dose due to SRS operations (see Table 4-14).

\section{Plutonium}

Plutonium at SRS was formed during the irradiation of nuclear fuel and targets during operation of the Site's five production reactors. The role of these reactors was to produce nuclear materials-principally tritium and ${ }^{239} \mathrm{Pu}-$ for national defense purposes. Additional radionuclides, such as ${ }^{238} \mathrm{Pu}$, which is a power source for certain deepspace missions, occasionally were produced for other government purposes.

Atmospheric plutonium releases occurred primarily in $\mathrm{F}$ Area and $\mathrm{H}$ Area and were largest during startup of the canyon facilities in 1955. Unidentified alpha releases from the reactors and other facilities were assumed to be plutonium. Approximately $70 \%$ of atmospheric plutonium releases occurred in 1955. From 1955 through 1996, the dose to the maximally exposed individual was $12 \mathrm{mrem}$, and the population dose was 710 person-rem (see Table 415).

There have been very few reports of plutonium releases to streams. Most of the releases reported in Table 4-16 are unidentified alpha releases; thus, the doses from liquid plutonium releases are much more uniform from year to year than atmospheric doses.

The dose to the maximally exposed individual was 0.28 mrem, with drinking water accounting for most of the dose. The total population dose was 12 person-rem. Overall, plutonium releases accounted for $15 \%$ of the population dose from SRS operations.

\section{Selected Fission Products}

The principal mechanism for production of ${ }^{95} \mathrm{Zr}, \mathrm{Nb}$, ${ }^{106} \mathrm{Ru}$, and ${ }^{144} \mathrm{Ce}$ was neutron-induced fission in the reactors. When a reactor was operating, neutron-induced fission reactions occurred in the ${ }^{235} \mathrm{U}$ fuel of the reactor core. Fission reactions formed a variety of fission products, which included those listed above.

Fission products rarely were seen in atmospheric releases from the reactors. Most fission products released to the atmosphere resulted from the separation process in F Area 
and $\mathrm{H}$ Area. In contrast, most of the fission products released to streams came from basin purges in the reactor areas.

\section{Zirconium, Niobium-95}

The dose from liquid releases of $130 \mathrm{Ci}$ of ${ }^{95} \mathrm{Zr}, \mathrm{Nb}$ was $15 \mathrm{mrem}$ to the maximally exposed individual, and drinking water doses at the water treatment plants were less than $0.03 \mathrm{mrem}$. The population dose, 25 personrem, resulted primarily from eating fish and saltwater invertebrates and occurred primarily in the 1960s (see Table 4-17). Overall, ${ }^{95} \mathrm{Zr}, \mathrm{Nb}$ releases accounted for $0.5 \%$ of the population dose from Site operations.

\section{Ruthenium-106}

Approximately $140 \mathrm{Ci}$ of ${ }^{106} \mathrm{Ru}$ were released to the atmosphere from 1955 through 1996. The dose to the maximally exposed individual at the Site boundary was about $4.4 \mathrm{mrem}$, and the population dose was 120 person-rem (see Table 4-18).

The dose from liquid releases of $59 \mathrm{Ci}$ of ${ }^{106} \mathrm{Ru}$ was $0.11 \mathrm{mrem}$ to the maximally exposed individual, and drinking water doses at the water treatment plants were less than $0.1 \mathrm{mrem}$. The population dose, 14 personrem, resulted primarily from the consumption of fish and saltwater invertebrates, and occurred primarily in the 1960s (see Table 4-19). Overall, 106Ru releases accounted for $2.7 \%$ of the population dose from Site operations.

\section{Cerium-144}

The dose from liquid releases of $350 \mathrm{Ci}$ of ${ }^{144} \mathrm{Ce}$ was 0.47 mrem to the maximally exposed individual, and drinking water doses at the water treatment plants were less than $0.4 \mathrm{mrem}$. The population dose, 47 personrem, resulted primarily from the consumption of fish and saltwater invertebrates and occurred primarily in the 1960s (see Table 4-20). Cerium-144 releases accounted for $1.0 \%$ of the population dose from Site operations.

\section{Strontium}

The principal mechanism for production of strontium was neutron-induced fission in the reactors. When a reactor was operating, neutron-induced fission reactions occurred in the ${ }^{235} \mathrm{U}$ fuel of the reactor core. Fission reactions formed a variety of fission products, of which strontium was one of the most important.

Strontium was not observed in atmospheric releases from the reactors. Most strontium released to the atmosphere came from the separation process in F Area and $\mathrm{H}$ Area. In contrast, most of the strontium released to streams came from basin purges in the reactor areas. Releases of unidentified beta-gamma occurred primarily from A Area and were assumed to be ${ }^{90} \mathrm{Sr}$ for dose calculations.

Approximately $3 \mathrm{Ci}$ of strontium were released to the atmosphere from 1955 through 1996 . The dose to the maximally exposed individual at the Site boundary was about 7.2 mrem, and the population dose was 6.3 person-rem (see Table 4-21).

Unidentified beta-gamma releases were assumed to be ${ }^{90} \mathrm{Sr}$ for both atmospheric and liquid releases. If a release point was monitored for ${ }^{90} \mathrm{Sr}$, the unidentified beta-gamma release was not included. A few ten of curies of release are thus not included in this summary total, but they are believed to be short half-life fission products, which would not contributed significantly to the total dose.

The dose from liquid releases of $150 \mathrm{Ci}$ of strontium was $2.5 \mathrm{mrem}$ to the maximally exposed individual, and drinking water doses at the water treatment plants were $1.3 \mathrm{mrem}$ or less. The population dose, 24 person-rem, was evenly divided among drinking water at Beaufort-Jasper and at Port Wentworth, and the consumption of fish and saltwater invertebrates (see Table 4-22). Strontium releases accounted for $0.6 \%$ of the population dose from Site operations. 


\section{Technetium}

There were virtually no measurements of ${ }^{99} \mathrm{Tc}$ releases. Release quantities have been conservatively estimated. Approximately $11 \mathrm{Ci}$ of ${ }^{99} \mathrm{Tc}$ were released to the atmosphere from 1955 through 1996. The dose to the maximally exposed individual at the Site boundary was about 0.1 mrem, and the population dose was 6.5 person-rem.

The dose from liquid releases of $53 \mathrm{Ci}$ of ${ }^{99} \mathrm{Tc}$ was less than 0.01 mrem to the maximally exposed individual, and drinking water doses at the water treatment plants also were less than 0.01 mrem. The population dose was 0.2 person-rem. Total ${ }^{99} \mathrm{Tc}$ releases accounted for $0.1 \%$ of the population dose from Site operations.

\section{Tritium}

Tritium was one of the principal products at SRS. It was produced in reactors in lithium-aluminum targets subjected to intense neutron irradiation. The targets were processed and the tritium packaged for shipment to other DOE facilities. A second (and undesirable) method of tritium production occurred when neutrons interacted with the heavy water moderator in the reactors. This tritium was the principal source of liquid releases and a significant contributor to atmospheric releases. A third method of tritium production, discovered at SRS, was ternary fission (Albenesius 1959). The uranium atom occasionally split into three pieces, one of which was tritium.

Approximately $2.6 \times 10^{7}$ million $\mathrm{Ci}$ of tritium were released to the atmosphere from 1955 through 1996 . The dose to the maximally exposed individual at the Site boundary was about 18 mrem, and the population dose was 1,100 personrem (see Table 4-23).

The dose from liquid releases of $1.6 \times 10^{6} \mathrm{Ci}$ of tritium was 7.9 mrem to the maximally exposed individual, and drinking water doses at the water treatment plants were $6.1 \mathrm{mrem}$ or less (see Table 4-24). The population dose was 130 person-rem-almost all of it due to drinking water at BeaufortJasper and at Port Wentworth (see Table 4-25). Tritium releases accounted for more than $25 \%$ of the population dose from Site operations.

\section{Uranium}

Uranium releases generally have been associated with the fabrication of reactor fuel and target elements (M Area) and with the chemical processing of spent target and fuel material (F Area and H Area).

Approximately $0.9 \mathrm{Ci}$ of uranium were released to the atmosphere from 1955 through 1996. The dose to the maximally exposed individual at the Site boundary was about 0.4 mrem, and the population dose was 32 person-rem (see Table 4-26).

The dose from liquid releases of $25 \mathrm{Ci}$ of uranium was 0.5 mrem to the maximally exposed individual, and drinking water doses at the water treatment plants were 0.4 mrem or less. The population dose was 8 person-rem-almost all of it due to drinking water at Beaufort-Jasper and at Port Wentworth (see Table 4-27). Uranium releases accounted for less than $1 \%$ of the population dose from Site operations.

\section{Site}

The total dose to the maximally exposed individual from all atmospheric releases (1954-1996) was 77 mrem (see Table 4-28). Iodine-131, tritium, and plutonium were the largest contributors. The population dose was 3,200 person-rem, with tritium, ${ }^{131} \mathrm{I}$, and plutonium contributing the most (see Table 4-29).

The total dose to the maximally exposed individual from all liquid releases was 140 mrem (see Table 4-30). Cesium$137,{ }^{32} \mathrm{P}$, and $95 \mathrm{Zr}, \mathrm{Nb}$ were the largest contributors. The drinking water dose at Beaufort-Jasper was $4 \mathrm{mrem}$, with tritium contributing most of the dose (see Table 4-31). The drinking water dose at Port Wentworth was $10 \mathrm{mrem}$, with tritium again contributing most of the dose (see Table 4-32).

The population dose at Beaufort-Jasper was 110 personrem, with tritium contributing most of the dose (see Table 433). The population dose at Port Wentworth was 75 person- 
rem, with tritium again contributing most of the dose (see Table 4-34). The population dose to the $80-\mathrm{km}$ population (Beaufort-Jasper and Port Wentworth not included) was 1,500 person-rem, with ${ }^{65} \mathrm{Zn},{ }^{137} \mathrm{Cs}$, and ${ }^{32} \mathrm{P}$ contributing the most (see Table 4-35). The sum of these three is the total population dose from liquid releases: 1,700 personrem. The largest contributors were ${ }^{65} \mathrm{Zn},{ }^{137} \mathrm{Cs}$, and tritium (see Table 4-36).

Combining the population doses from both atmospheric and liquid releases results in a total population dose from SRS operations from 1954 through 1996 of 4,800 personrem (see Table 4-37). The principal contributors were tritium $(25 \%),{ }^{65} \mathrm{Zn}(23 \%),{ }^{131} \mathrm{I}(18 \%)$, and plutonium (15\%) (see Table 4-38). The largest annual population dose, 580 person-rem, occurred in 1955 (see Table 4-39).

\section{References for Chapter 4}

Albenesius, E.L., 1959, "Tritium as a Product of Fission", Phy. Rev. Let. 3(6):274.

Angerman, C.L., 1973, Savannah River Laboratory Cobalt-60 Power and Heat Sources, Final Quarterly Progress Report, DP-1338, E.I. duPont de Nemours \& Co., Aiken, South Carolina.

Arnett, M.W., L.K. Karapatakis, A.R. Mamatey, and J.L. Todd, 1992, Savannah River Site Environmental Report for 1991, WSRC-TR-92-186, Westinghouse Savannah River Company, Aiken, South Carolina.

Arnett, M.W., L.K. Karapatakis, and A.R. Mamatey, 1993, Savannah River Site Environmental Report for 1992, WSRC-TR-93-075, Westinghouse Savannah River Company, Aiken, South Carolina.

Arnett, M.W., L.K. Karapatakis, and A.R. Mamatey, 1994, Savannah River Site Environmental Report for 1993, WSRC-TR-94-075, Westinghouse Savannah River Company, Aiken, South Carolina.

Arnett, M.W., A.R. Mamatey, and D. Spitzer, 1995, Savannah River Site Environmental Report for 1994, WSRC-TR-95-075, Westinghouse Savannah River Company, Aiken, South Carolina.

Arnett, M.W., and A.R. Mamatey, 1996, Savannah River Site Environmental Report for 1995, WSRC-TR-96-
0075, Westinghouse Savannah River Company, Aiken, South Carolina.

Arnett, M.W., and A.R. Mamatey, 1997, Savannah River Site Environmental Report for 1996, WSRC-TR-970171, Westinghouse Savannah River Company, Aiken, South Carolina.

Ashley, C., 1966, Environmental Monitoring at the Savannah River Plant, Annual Report-1966, DPST-67-302, E.I. duPont de Nemours \& Co., Aiken, South Carolina.

Bebbington, W.P., 1990, History of Du Pont at the Savannah River Plant, E.I. duPont de Nemours \& Co., Aiken, South Carolina.

Cummins, C.L., D.K. Martin, and J.L. Todd, 1991a, Savannah River Site Environmental Report, 1990, WSRC-IM-91-28, Westinghouse̊̀ Savannah River Company, Aiken, South Carolina.

Cummins, C.L., C.S. Hetrick, and D.K. Martin, 1991b, Radioactive Releases at the Savannah River Site 1954-1989, WSRC-RP-91-684, Westinghouse Savannah River Company, Aiken, South Carolina.

Fox, L.W., 1975, Radioactive Releases in Excess of Annual Guides, SRT-ETS-960031, E.I. duPont de Nemours \& Co., Aiken, South Carolina.

Hayes, D.W., and K.W. MacMurdo, 1977, "Carbon-14 Production by the Nuclear Industry," Health Physics, 32: 215-219.

Longtin, F.B., 1966, Moderator Silicate Control of ${ }^{32 P}$ Release, DPSOX-6546, E.I. duPont de Nemours \& Co., Aiken, SC.

Longtin, F.B., 1972, 100-Area Release Guides for ${ }^{95} \mathrm{Zr}-\mathrm{Nb}$ and ${ }^{51} \mathrm{Cr}$, SRT-ETS-960029, Westinghouse Savannah River Company, Aiken, South Carolina.

Moyer, R.A., 1968, "Savannah River Experience with Transplutonium Elements," Health Physics 15: 133 138.

Stoddard, D.H., 1964, Radiation Properties of ${ }^{244} \mathrm{Cm}$ Produced for Isotopic Power Generators, DP-939, E.I. du Pont de Nemours \& Company, Savannah River Laboratory, Aiken, SC.

Zecha, D.J., 1987, ${ }^{60}$ Co Fueled Thermoelectric Generator Burial, DPSOX-10051, E.I. duPont de Nemours \& Co., Aiken, SC. 
Table 4-1. Liquid P-32 Releases and Dose

\begin{tabular}{|c|c|c|c|c|c|c|c|c|c|c|c|}
\hline Year & $\begin{array}{l}\text { River } \\
\text { Flow } \\
\text { Rate } \\
\left(\mathrm{m}^{3} / \mathrm{s}\right)\end{array}$ & \begin{tabular}{|c|} 
BJ \\
Derived \\
River \\
Flow \\
Rate \\
$\left(\mathrm{m}^{3} / \mathrm{s}\right)$ \\
\end{tabular} & \begin{tabular}{|c|} 
PW \\
Derived \\
River \\
Flow \\
Rate \\
$\left(\mathrm{m}^{3} / \mathrm{s}\right)$
\end{tabular} & $\begin{array}{c}\text { Releases } \\
\text { to } \\
\text { Streams } \\
\text { (Ci) }\end{array}$ & $\begin{array}{c}\text { Max } \\
\text { Ind } \\
\text { Below } \\
\text { SRS } \\
\text { (mrem) }\end{array}$ & $\begin{array}{c}\text { Max } \\
\text { Ind } \\
\text { BJ } \\
\text { (mrem) }\end{array}$ & $\begin{array}{c}\text { Max } \\
\text { Ind } \\
\text { PW } \\
\text { (mrem) }\end{array}$ & $\begin{array}{c}\text { Pop } \\
\text { Dose } \\
\text { BJ } \\
\text { (per-rem) }\end{array}$ & $\begin{array}{c}\text { Pop } \\
\text { Dose } \\
\text { PW } \\
\text { (per-rem) }\end{array}$ & $\begin{array}{c}\text { Pop } \\
\text { Dose } \\
80 \mathrm{~km} \\
\text { (per-rem) }\end{array}$ & $\begin{array}{c}\text { Pop } \\
\text { Dose } \\
\text { Total } \\
\text { (per-rem) }\end{array}$ \\
\hline 1954 & 209 & & 263 & & & & & & & & \\
\hline 1955 & 169 & & 213 & & & & & & & & \\
\hline 1956 & 179 & & 225 & & & & & & & & \\
\hline 1957 & 235 & & 297 & & & & & & & & \\
\hline 1958 & 313 & & 394 & & & & & & & & \\
\hline 1959 & 276 & & 348 & & & & & & & & \\
\hline 1960 & 371 & & 468 & & & & & & & & \\
\hline 1961 & 309 & & 389 & & & & & & & & \\
\hline 1962 & 300 & & 377 & & & & & & & & \\
\hline 1963 & 315 & & 397 & & & & & & & & \\
\hline 1964 & 580 & & 731 & $3.1 \times 10^{0}$ & $2.2 \times 10^{0}$ & & $7.0 \times 10^{-4}$ & & $4.6 \times 10^{-3}$ & $5.4 \times 10^{0}$ & $5.4 \times 10^{0}$ \\
\hline 1965 & 362 & 800 & 456 & $1.4 \times 10^{1}$ & $1.6 \times 10^{1}$ & $2.8 \times 10^{-3}$ & $5.0 \times 10^{-3}$ & $6.3 \times 10^{-2}$ & $3.3 \times 10^{-2}$ & $3.8 \times 10^{1}$ & $3.8 \times 10^{1}$ \\
\hline 1966 & 316 & 520 & 399 & $1.0 \times 10^{1}$ & $1.4 \times 10^{1}$ & $3.3 \times 10^{-3}$ & $4.3 \times 10^{-3}$ & $7.3 \times 10^{-2}$ & $2.8 \times 10^{-2}$ & $3.3 \times 10^{1}$ & $3.3 \times 10^{1}$ \\
\hline 1967 & 299 & 625 & 377 & $4.2 \times 10^{0}$ & $5.9 \times 10^{0}$ & $1.1 \times 10^{-3}$ & $1.8 \times 10^{-3}$ & $2.4 \times 10^{-2}$ & $1.2 \times 10^{-2}$ & $1.4 \times 10^{1}$ & $1.4 \times 10^{1}$ \\
\hline 1968 & 273 & 470 & 343 & $2.4 \times 10^{0}$ & $3.8 \times 10^{0}$ & $8.7 \times 10^{-4}$ & $1.2 \times 10^{-3}$ & $1.9 \times 10^{-2}$ & $7.9 \times 10^{-3}$ & $9.1 \times 10^{0}$ & $9.1 \times 10^{0}$ \\
\hline 1969 & 310 & 624 & 390 & $7.3 \times 10^{-1}$ & $1.0 \times 10^{0}$ & $2.0 \times 10^{-4}$ & $3.1 \times 10^{-4}$ & $4.3 \times 10^{-3}$ & $2.1 \times 10^{-3}$ & $2.4 \times 10^{0}$ & $2.4 \times 10^{0}$ \\
\hline 1970 & 232 & 772 & 293 & $7.7 \times 10^{-1}$ & $1.4 \times 10^{0}$ & $1.7 \times 10^{-4}$ & $4.4 \times 10^{-4}$ & $3.7 \times 10^{-3}$ & $2.9 \times 10^{-3}$ & $3.4 \times 10^{0}$ & $3.4 \times 10^{0}$ \\
\hline 1971 & 303 & 1245 & 381 & $9.2 \times 10^{-1}$ & $1.3 \times 10^{0}$ & $1.2 \times 10^{-4}$ & $4.0 \times 10^{-4}$ & $2.7 \times 10^{-3}$ & $2.7 \times 10^{-3}$ & $3.1 \times 10^{0}$ & $3.1 \times 10^{0}$ \\
\hline 1972 & 318 & 775 & 401 & $3.0 \times 10^{-1}$ & $4.0 \times 10^{-1}$ & $6.5 \times 10^{-5}$ & $1.2 \times 10^{-4}$ & $1.4 \times 10^{-3}$ & $8.3 \times 10^{-4}$ & $9.6 \times 10^{-1}$ & $9.6 \times 10^{-1}$ \\
\hline 1973 & 409 & 656 & 468 & $1.3 \times 10^{-1}$ & $1.4 \times 10^{-1}$ & $3.4 \times 10^{-5}$ & $4.8 \times 10^{-5}$ & $7.5 \times 10^{-4}$ & $3.1 \times 10^{-4}$ & $3.3 \times 10^{-1}$ & $3.3 \times 10^{-1}$ \\
\hline 1974 & 314 & 640 & 373 & $1.4 \times 10^{-2}$ & $1.9 \times 10^{-2}$ & $3.7 \times 10^{-6}$ & $6.3 \times 10^{-6}$ & $8.0 \times 10^{-5}$ & $4.1 \times 10^{-5}$ & $4.5 \times 10^{-2}$ & $4.6 \times 10^{-2}$ \\
\hline 1975 & 436 & 877 & 538 & $2.0 \times 10^{-3}$ & $1.9 \times 10^{-3}$ & $3.8 \times 10^{-7}$ & $6.2 \times 10^{-7}$ & $8.4 \times 10^{-6}$ & $4.1 \times 10^{-6}$ & $4.7 \times 10^{-3}$ & $4.7 \times 10^{-3}$ \\
\hline 1976 & 394 & 996 & 472 & $1.7 \times 10^{-2}$ & $1.8 \times 10^{-2}$ & $2.8 \times 10^{-6}$ & $6.0 \times 10^{-6}$ & $6.3 \times 10^{-5}$ & $4.0 \times 10^{-5}$ & $4.4 \times 10^{-2}$ & $4.4 \times 10^{-2}$ \\
\hline 1977 & 330 & 562 & 433 & $6.4 \times 10^{-3}$ & $8.3 \times 10^{-3}$ & $1.9 \times 10^{-6}$ & $2.5 \times 10^{-6}$ & $4.2 \times 10^{-5}$ & $1.6 \times 10^{-5}$ & $2.0 \times 10^{-2}$ & $2.0 \times 10^{-2}$ \\
\hline 1978 & 298 & 689 & 324 & & & & & & & & \\
\hline 1979 & 375 & 989 & 495 & $4.0 \times 10^{-3}$ & $4.5 \times 10^{-3}$ & $2.4 \times 10^{-5}$ & $1.3 \times 10^{-6}$ & $1.5 \times 10^{-5}$ & $8.9 \times 10^{-6}$ & $1.1 \times 10^{-2}$ & $1.1 \times 10^{-2}$ \\
\hline 1980 & 374 & 863 & 488 & & & & & & & & \\
\hline 1981 & 187 & 331 & 243 & & & & & & & & \\
\hline 1982 & 203 & 555 & 287 & & & & & & & & \\
\hline 1983 & 350 & 361 & 417 & & & & & & & & \\
\hline 1984 & 361 & 747 & 487 & & & & & & & & \\
\hline 1985 & 203 & 263 & 254 & & & & & & & & \\
\hline 1986 & 175 & 235 & 213 & & & & & & & & \\
\hline 1987 & 254 & 374 & 357 & & & & & & & & \\
\hline 1988 & 152 & 211 & 203 & & & & & & & & \\
\hline 1989 & 226 & 240 & 245 & & & & & & & & \\
\hline 1990 & 336 & 447 & 367 & $2.6 \times 10^{-3}$ & $3.3 \times 10^{-3}$ & $9.7 \times 10^{-7}$ & $1.2 \times 10^{-6}$ & $2.1 \times 10^{-5}$ & $7.8 \times 10^{-6}$ & $7.9 \times 10^{-3}$ & $7.9 \times 10^{-3}$ \\
\hline 1991 & 328 & 550 & 453 & & & & & & & & \\
\hline 1992 & 331 & 350 & 279 & & & & & & & & \\
\hline 1993 & 419 & 566 & 416 & & & & & & & & \\
\hline 1994 & 347 & 421 & 348 & & & & & & & & \\
\hline 1995 & 361 & 438 & 369 & & & & & & & & \\
\hline 1996 & 325 & 310 & 287 & & & & & & & & \\
\hline Total & & & & $3.6 \times 10^{1}$ & $4.6 \times 10^{1}$ & $8.7 \times 10^{-3}$ & $1.4 \times 10^{-2}$ & $1.9 \times 10^{-1}$ & $9.5 \times 10^{-2}$ & $1.1 \times 10^{2}$ & $1.1 \times 10^{2}$ \\
\hline
\end{tabular}


Table 4-2. Liquid Cr-51 Releases and Dose

\begin{tabular}{|c|c|c|c|c|c|c|c|c|c|c|c|}
\hline Year & $\begin{array}{c}\text { River } \\
\text { Flow Rate } \\
\left(\mathrm{m}^{3} / \mathrm{s}\right)\end{array}$ & $\begin{array}{c}\text { B-J } \\
\text { Derived } \\
\text { Flow } \\
\text { Rate } \\
\left(\mathrm{m}^{3} / \mathrm{s}\right)\end{array}$ & $\begin{array}{c}\text { PW } \\
\text { Derived } \\
\text { Flow } \\
\text { Rate } \\
\left(\mathrm{m}^{3} / \mathrm{s}\right)\end{array}$ & $\begin{array}{c}\text { Releases } \\
\text { to } \\
\text { Streams } \\
\text { (Ci) }\end{array}$ & $\begin{array}{l}\text { Max } \\
\text { Ind } \\
\text { Below } \\
\text { SRS } \\
\text { (mrem) }\end{array}$ & $\begin{array}{c}\text { Max } \\
\text { Ind } \\
\text { BJ } \\
\text { (mrem) }\end{array}$ & $\begin{array}{c}\text { Max } \\
\text { Ind } \\
\text { PW } \\
\text { (mrem) }\end{array}$ & $\begin{array}{c}\text { Pop } \\
\text { Dose } \\
\text { BJ } \\
\text { (per-rem) }\end{array}$ & \begin{tabular}{|c} 
Pop \\
Dose \\
PW \\
(per-rem)
\end{tabular} & $\begin{array}{c}\text { Pop } \\
\text { Dose } \\
80 \mathrm{~km} \\
\text { (per-rem) }\end{array}$ & $\begin{array}{c}\text { Pop } \\
\text { Dose } \\
\text { Total } \\
\text { (per-rem) }\end{array}$ \\
\hline 1954 & 209 & & 263 & & & & & & & & \\
\hline 1955 & 169 & & 213 & & & & & & & & \\
\hline 1956 & 179 & & 225 & & & & & & & & \\
\hline 1957 & 235 & & 297 & & & & & & & & \\
\hline 1958 & 313 & & 394 & & & & & & & & \\
\hline 1959 & 276 & & 348 & & & & & & & & \\
\hline 1960 & 371 & & 468 & $1.1 \times 10^{1}$ & $5.5 \times 10^{-4}$ & & $6.9 \times 10^{-5}$ & & $5.0 \times 10^{-4}$ & $2.3 \times 10^{-2}$ & $2.3 \times 10^{-2}$ \\
\hline 1961 & 309 & & 389 & $6.2 \times 10^{1}$ & $3.5 \times 10^{-3}$ & & $4.5 \times 10^{-4}$ & & $3.3 \times 10^{-3}$ & $1.5 \times 10^{-1}$ & $1.5 \times 10^{-1}$ \\
\hline 1962 & 300 & & 377 & $2.1 \times 10^{2}$ & $1.3 \times 10^{-2}$ & & $1.6 \times 10^{-3}$ & & $1.2 \times 10^{-2}$ & $5.2 \times 10^{-1}$ & $5.3 \times 10^{-1}$ \\
\hline 1963 & 315 & & 397 & $1.3 \times 10^{3}$ & $7.2 \times 10^{-2}$ & & $9.1 \times 10^{-3}$ & & $6.7 \times 10^{-2}$ & $3.0 \times 10^{\circ}$ & $3.1 \times 10^{0}$ \\
\hline 1964 & 580 & & 731 & $1.1 \times 10^{3}$ & $3.3 \times 10^{-2}$ & & $4.2 \times 10^{-3}$ & & $3.1 \times 10^{-2}$ & $1.4 \times 10^{0}$ & $1.4 \times 10^{0}$ \\
\hline 1965 & 362 & 800 & 456 & $7.8 \times 10^{2}$ & $3.8 \times 10^{-2}$ & $2.7 \times 10^{-3}$ & $4.8 \times 10^{-3}$ & $6.6 \times 10^{-2}$ & $3.5 \times 10^{-2}$ & $1.6 \times 10^{0}$ & $1.7 \times 10^{0}$ \\
\hline 1966 & 316 & 520 & 399 & $6.3 \times 10^{2}$ & $3.5 \times 10^{-2}$ & $3.4 \times 10^{-3}$ & $4.4 \times 10^{-3}$ & $8.2 \times 10^{-2}$ & $3.2 \times 10^{-2}$ & $1.5 \times 10^{0}$ & $1.6 \times 10^{0}$ \\
\hline 1967 & 299 & 625 & 377 & $2.4 \times 10^{2}$ & $1.5 \times 10^{-2}$ & $1.1 \times 10^{-3}$ & $1.8 \times 10^{-3}$ & $2.6 \times 10^{-2}$ & $1.3 \times 10^{-2}$ & $6.0 \times 10^{-1}$ & $6.4 \times 10^{-1}$ \\
\hline 1968 & 273 & 470 & 343 & $8.7 \times 10^{1}$ & $5.7 \times 10^{-3}$ & $5.2 \times 10^{-4}$ & $7.1 \times 10^{-4}$ & $1.3 \times 10^{-2}$ & $5.2 \times 10^{-3}$ & $2.3 \times 10^{-1}$ & $2.5 \times 10^{-4}$ \\
\hline 1969 & 310 & 624 & 390 & $6.8 \times 10^{1}$ & $3.9 \times 10^{-3}$ & $3.1 \times 10^{-4}$ & $4.9 \times 10^{-4}$ & $7.4 \times 10^{-3}$ & $3.6 \times 10^{-3}$ & $1.6 \times 10^{-1}$ & $1.7 \times 10^{-1}$ \\
\hline 1970 & 232 & 772 & 293 & $1.6 \times 10^{1}$ & $1.2 \times 10^{-3}$ & $5.9 \times 10^{-5}$ & $1.5 \times 10^{-4}$ & $1.4 \times 10^{-3}$ & $1.1 \times 10^{-3}$ & $5.1 \times 10^{-2}$ & $5.3 \times 10^{-2}$ \\
\hline 1971 & 303 & 1245 & 381 & $7.4 \times 10^{0}$ & $4.3 \times 10^{-4}$ & $1.7 \times 10^{-5}$ & $5.5 \times 10^{-5}$ & $4.0 \times 10^{-4}$ & $4.0 \times 10^{-4}$ & $1.8 \times 10^{-2}$ & $1.9 \times 10^{-2}$ \\
\hline 1972 & 318 & 775 & 401 & $1.4 \times 10^{\circ}$ & $7.7 \times 10^{-5}$ & $5.0 \times 10^{-6}$ & $9.7 \times 10^{-6}$ & $1.2 \times 10^{-4}$ & $7.1 \times 10^{-5}$ & $3.2 \times 10^{-3}$ & $3.4 \times 10^{-3}$ \\
\hline 1973 & 409 & 656 & 468 & $4.4 \times 10^{-1}$ & $1.9 \times 10^{-5}$ & $1.9 \times 10^{6}$ & $2.7 \times 10^{-6}$ & $4.6 \times 10^{-5}$ & $1.9 \times 10^{-5}$ & $7.9 \times 10^{-4}$ & $8.6 \times 10^{-4}$ \\
\hline 1974 & 314 & 640 & 373 & $6.0 \times 10^{-1}$ & $3.4 \times 10^{-5}$ & $2.7 \times 10^{-6}$ & $4.6 \times 10^{-6}$ & $6.4 \times 10^{-5}$ & $3.3 \times 10^{-5}$ & $1.4 \times 10^{-3}$ & $1.5 \times 10^{-3}$ \\
\hline 1975 & 436 & 877 & 538 & $1.2 \times 10^{-1}$ & $4.7 \times 10^{-6}$ & $3.7 \times 10^{-7}$ & $6.1 \times 10^{-7}$ & $9.0 \times 10^{-6}$ & $4.5 \times 10^{-6}$ & $2.0 \times 10^{-4}$ & $2.1 \times 10^{-4}$ \\
\hline 1976 & 394 & 996 & 472 & $3.6 \times 10^{-1}$ & $1.6 \times 10^{-5}$ & $1.0 \times 10^{-6}$ & $2.2 \times 10^{-6}$ & $2.5 \times 10^{-5}$ & $1.6 \times 10^{-5}$ & $6.7 \times 10^{-4}$ & $7.1 \times 10^{-4}$ \\
\hline 1977 & 330 & 562 & 433 & $6.6 \times 10^{-1}$ & $3.6 \times 10^{-5}$ & $3.3 \times 10^{-6}$ & $4.3 \times 10^{-6}$ & $8.0 \times 10^{-5}$ & $3.1 \times 10^{-5}$ & $1.5 \times 10^{-3}$ & $1.6 \times 10^{-3}$ \\
\hline 1978 & 298 & 689 & 324 & $4.0 \times 10^{-2}$ & $2.4 \times 10^{-6}$ & $1.6 \times 10^{-7}$ & $3.5 \times 10^{-7}$ & $3.9 \times 10^{-6}$ & $2.6 \times 10^{-6}$ & $9.9 \times 10^{-5}$ & $1.1 \times 10^{-4}$ \\
\hline 1979 & 375 & 989 & 495 & $5.4 \times 10^{-1}$ & $2.6 \times 10^{-5}$ & $1.5 \times 10^{-6}$ & $3.1 \times 10^{-6}$ & $3.7 \times 10^{-5}$ & $2.3 \times 10^{-5}$ & $1.1 \times 10^{-3}$ & $1.1 \times 10^{-3}$ \\
\hline 1980. & 374 & 863 & 488 & & & & & & & & \\
\hline 1981 & 187 & 331 & 243 & & & 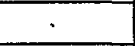 & & & & & \\
\hline 1982 & 203 & 555 & 287 & & & & & & & & \\
\hline 1983 & 350 & 361 & 417 & & & & & & & & \\
\hline 1984 & 361 & 747 & 487 & & & & & & & & \\
\hline 1985 & 203 & 263 & 254 & & & & & & & & \\
\hline 1986 & 175 & 235 & 213 & & & & & & & & \\
\hline 1987 & 254 & 374 & 357 & & & & & & & & \\
\hline 1988 & 152 & 211 & 203 & & & & & & & & \\
\hline 1989 & 226 & 240 & 245 & & & & & & & & . \\
\hline 1990 & 336 & 447 & 367 & & & & & & & & \\
\hline 1991 & 328 & 550 & 453 & & & & & & & & \\
\hline 1992 & 331 & 350 & 279 & & & & & & & & \\
\hline 1993 & 419 & 566 & 416 & & & & & & & & \\
\hline 1994 & 347 & 421 & 348 & & & & & & & & \\
\hline 1995 & 361 & 438 & 369 & & & & & & & & \\
\hline 1996 & 325 & 310 & 287 & & & & & & & & \\
\hline Total & & & & $4.5 \times 10^{3}$ & $2.2 \times 10^{-1}$ & $8.2 \times 10^{-3}$ & $2.8 \times 10^{-2}$ & $2.0 \times 10^{-1}$ & $2.0 \times 10^{-1}$ & $9.2 \times 10^{0}$ & $9.6 \times 10^{0}$ \\
\hline
\end{tabular}


Table 4-3. Atmospheric Co-60 Releases and Dose

\begin{tabular}{|c|c|c|c|c|c|}
\hline Year & $\begin{array}{l}\text { Release } \\
\text { A Area } \\
\text { (Ci) }\end{array}$ & $\begin{array}{c}\text { Release } \\
\text { Reactors and } \\
\text { Separations } \\
\text { (Ci) }\end{array}$ & $\begin{array}{c}\text { Total } \\
\text { Release } \\
\text { (Ci) }\end{array}$ & $\begin{array}{l}\text { Maximum } \\
\text { Individual } \\
\text { Dose } \\
\text { at Boundary } \\
\text { (mrem) }\end{array}$ & $\begin{array}{c}\text { Population } \\
\text { Dose } \\
\text { (per-rem) }\end{array}$ \\
\hline \multicolumn{6}{|l|}{1954} \\
\hline \multicolumn{6}{|l|}{1955} \\
\hline \multicolumn{6}{|l|}{1956} \\
\hline \multicolumn{6}{|l|}{1957} \\
\hline \multicolumn{6}{|l|}{1958} \\
\hline \multicolumn{6}{|l|}{1959} \\
\hline \multicolumn{6}{|l|}{1960} \\
\hline \multicolumn{6}{|l|}{1961} \\
\hline \multicolumn{6}{|l|}{1962} \\
\hline \multicolumn{6}{|l|}{1963} \\
\hline \multicolumn{6}{|l|}{1964} \\
\hline \multicolumn{6}{|l|}{1965} \\
\hline \multicolumn{6}{|l|}{1966} \\
\hline \multicolumn{6}{|l|}{1967} \\
\hline 1968 & $3.9 \times 10^{-2}$ & & $3.9 \times 10^{-2}$ & $4.1 \times 10^{-1}$ & $2.8 \times 10^{-1}$ \\
\hline 1969 & $1.1 \times 10^{-2}$ & & $1.1 \times 10^{-2}$ & $1.2 \times 10^{-1}$ & $7.8 \times 10^{-2}$ \\
\hline 1970 & $2.8 \times 10^{-3}$ & & $2.8 \times 10^{-3}$ & $3.0 \times 10^{-2}$ & $2.0 \times 10^{-2}$ \\
\hline 1971 & $2.6 \times 10^{-3}$ & $2.0 \times 10^{-2}$ & $2.3 \times 10^{-2}$ & $2.9 \times 10^{-2}$ & $1.6 \times 10^{-1}$ \\
\hline 1972 & $7.1 \times 10^{-3}$ & $9.0 \times 10^{-5}$ & $7.2 \times 10^{-3}$ & $7.5 \times 10^{-2}$ & $5.1 \times 10^{-2}$ \\
\hline 1973 & $1.8 \times 10^{-3}$ & & $1.8 \times 10^{-3}$ & $1.9 \times 10^{-2}$ & $1.3 \times 10^{-2}$ \\
\hline 1974 & $3.9 \times 10^{-3}$ & & $3.9 \times 10^{-3}$ & $4.1 \times 10^{-2}$ & $2.8 \times 10^{-2}$ \\
\hline 1975 & $1.1 \times 10^{-3}$ & & $1.1 \times 10^{-3}$ & $1.2 \times 10^{-2}$ & $7.8 \times 10^{-3}$ \\
\hline 1976 & $1.3 \times 10^{-4}$ & & $1.3 \times 10^{-4}$ & $1.4 \times 10^{-3}$ & $9.3 \times 10^{-4}$ \\
\hline 1977 & $3.8 \times 10^{-4}$ & & $3.8 \times 10^{-4}$ & $4.0 \times 10^{-3}$ & $2.7 \times 10^{-3}$ \\
\hline 1978 & $3.8 \times 10^{-4}$ & & $3.8 \times 10^{-4}$ & $4.0 \times 10^{-3}$ & $2.7 \times 10^{-3}$ \\
\hline 1979 & $4.0 \times 10^{-4}$ & & $4.0 \times 10^{-4}$ & $4.2 \times 10^{-3}$ & $2.8 \times 10^{-3}$ \\
\hline 1980 & $6.2 \times 10^{-4}$ & & $6.2 \times 10^{-4}$ & $6.6 \times 10^{-3}$ & $4.4 \times 10^{-3}$ \\
\hline 1981 & $8.9 \times 10^{.5}$ & & $8.9 \times 10^{-5}$ & $9.4 \times 10^{-4}$ & $6.3 \times 10^{-4}$ \\
\hline 1982 & $4.4 \times 10^{-5}$ & & $4.4 \times 10^{-5}$ & $4.7 \times 10^{-4}$ & $3.1 \times 10^{-4}$ \\
\hline 1983 & $1.7 \times 10^{-4}$ & & $1.7 \times 10^{-4}$ & $1.8 \times 10^{-3}$ & $1.2 \times 10^{-3}$ \\
\hline 1984 & $5.4 \times 10^{-5}$ & & $5.4 \times 10^{-5}$ & $5.7 \times 10^{-4}$ & $3.8 \times 10^{-4}$ \\
\hline \multicolumn{6}{|l|}{1985} \\
\hline 1986 & & $8.0 \times 10^{-6}$ & $8.0 \times 10^{-6}$ & $1.1 \times 10^{-6}$ & $5.7 \times 10^{-5}$ \\
\hline \multicolumn{6}{|l|}{1987} \\
\hline 1988 & & $3.0 \times 10^{-6}$ & $3.0 \times 10^{-6}$ & $4.0 \times 10^{-7}$ & $2.1 \times 10^{-5}$ \\
\hline \multicolumn{6}{|l|}{1989} \\
\hline 1990 & & $4.8 \times 10^{-6}$ & $4.8 \times 10^{-6}$ & $6.3 \times 10^{-7}$ & $3.4 \times 10^{-5}$ \\
\hline 1991 & $3.8 \times 10^{-7}$ & $3.1 \times 10^{-6}$ & $3.5 \times 10^{-6}$ & $4.3 \times 10^{-6}$ & $2.5 \times 10^{-5}$ \\
\hline 1992 & & $3.6 \times 10^{-7}$ & $3.6 \times 10^{-7}$ & $4.8 \times 10^{-8}$ & $2.6 \times 10^{-6}$ \\
\hline 1993 & & $5.9 \times 10^{-9}$ & $5.9 \times 10^{-9}$ & $7.8 \times 10^{-10}$ & $4.2 \times 10^{-8}$ \\
\hline 1994 & $6.2 \times 10^{-6}$ & & $6.2 \times 10^{-6}$ & $6.6 \times 10^{-5}$ & $4.4 \times 10^{-5}$ \\
\hline 1995 & $2.5 \times 10^{-6}$ & $5.5 \times 10^{-5}$ & $5.8 \times 10^{-5}$ & $3.1 \times 10^{-5}$ & $4.1 \times 10^{-4}$ \\
\hline 1996 & $8.6 \times 10^{-6}$ & $8.6 \times 10^{-7}$ & $9.4 \times 10^{-6}$ & $9.1 \times 10^{-5}$ & $6.7 \times 10^{-5}$ \\
\hline Total & $7.2 \times 10^{-2}$ & $2.0 \times 10^{-2}$ & $9.2 \times 10^{-2}$ & $7.6 \times 10^{-1}$ & $6.5 \times 10^{-1}$ \\
\hline
\end{tabular}


Table 4-4. Liquid Co-60 Releases and Dose

\begin{tabular}{|c|c|c|c|c|c|c|c|c|c|c|c|}
\hline Year & $\begin{array}{c}\text { River } \\
\text { Flow } \\
\text { Rate } \\
\left(\mathrm{m}^{3} / \mathrm{s}\right)\end{array}$ & $\begin{array}{c}\text { BJ } \\
\text { Derived } \\
\text { Flow } \\
\text { Rate } \\
\left(\mathrm{m}^{3} / \mathrm{s}\right)\end{array}$ & $\begin{array}{c}\text { PW } \\
\text { Derived } \\
\text { Flow } \\
\text { Rate } \\
\left(\mathrm{m}^{3} / \mathrm{s}\right)\end{array}$ & $\begin{array}{l}\text { Releases } \\
\text { to } \\
\text { Streams } \\
\text { (Ci) }\end{array}$ & $\begin{array}{c}\text { Max } \\
\text { Ind } \\
\text { Below } \\
\text { SRS } \\
\text { (mrem) }\end{array}$ & $\begin{array}{c}\text { Max } \\
\text { Ind } \\
\text { BJ } \\
\text { (mrem) }\end{array}$ & $\begin{array}{c}\text { Max } \\
\text { Ind } \\
\text { PW } \\
\text { (mrem) }\end{array}$ & $\begin{array}{c}\text { Pop } \\
\text { Dose } \\
\text { BJ } \\
\text { (per-rem) }\end{array}$ & $\begin{array}{c}\text { Pop } \\
\text { Dose } \\
\text { PW } \\
\text { (per-rem) }\end{array}$ & $\begin{array}{c}\text { Pop } \\
\text { Dose } \\
80 \mathrm{~km} \\
\text { (per-rem) }\end{array}$ & $\begin{array}{c}\text { Pop } \\
\text { Dose } \\
\text { Total } \\
\text { (per-rem) }\end{array}$ \\
\hline 1954 & 209 & & 263 & $6.0 \times 10^{-2}$ & $6.1 \times 10^{-4}$ & & $1.4 \times 10^{-4}$ & & $1.0 \times 10^{-3}$ & $3.7 \times 10^{-2}$ & $3.8 \times 10^{-2}$ \\
\hline 1955 & 169 & & 213 & $2.2 \times 10^{-1}$ & $2.8 \times 10^{-3}$ & & $6.2 \times 10^{-4}$ & & $4.8 \times 10^{-3}$ & $1.7 \times 10^{-1}$ & $1.8 \times 10^{-1}$ \\
\hline 1956 & 179 & & 225 & $6.0 \times 10^{-1}$ & $7.1 \times 10^{-3}$ & & $1.6 \times 10^{-3}$ & & $1.2 \times 10^{-2}$ & $4.4 \times 10^{-1}$ & $4.5 \times 10^{-1}$ \\
\hline 1957 & 235 & & 297 & $1.6 \times 10^{0}$ & $1.5 \times 10^{-2}$ & & $3.3 \times 10^{-3}$ & & $2.5 \times 10^{-2}$ & $9.1 \times 10^{-1}$ & $9.4 \times 10^{-1}$ \\
\hline 1958 & 313 & & 394 & $1.1 \times 10^{0}$ & $7.3 \times 10^{-3}$ & & $1.6 \times 10^{-3}$ & & $1.2 \times 10^{-2}$ & $4.5 \times 10^{-1}$ & $4.6 \times 10^{-1}$ \\
\hline 1959 & 276 & & 348 & $2.6 \times 10^{0}$ & $2.0 \times 10^{-2}$ & & $4.5 \times 10^{-3}$ & & $3.4 \times 10^{-2}$ & $1.2 \times 10^{0}$ & $1.3 \times 10^{0}$ \\
\hline 1960 & 371 & & 468 & $7.8 \times 10^{0}$ & $4.5 \times 10^{-2}$ & & $9.9 \times 10^{-3}$ & & $7.5 \times 10^{-2}$ & $2.7 \times 10^{0}$ & $2.8 \times 10^{0}$ \\
\hline 1961 & 309 & & 389 & $6.4 \times 10^{0}$ & $4.4 \times 10^{-2}$ & & $9.8 \times 10^{-3}$ & & $7.5 \times 10^{-2}$ & $2.7 \times 10^{0}$ & $2.8 \times 10^{0}$ \\
\hline 1962 & 300 & & 377 & $1.3 \times 10^{1}$ & $9.0 \times 10^{-2}$ & & $2.0 \times 10^{-2}$ & & $1.5 \times 10^{-1}$ & $5.5 \times 10^{0}$ & $5.7 \times 10^{0}$ \\
\hline 1963 & 315 & & 397 & $5.1 \times 10^{0}$ & $3.4 \times 10^{-2}$ & & $7.6 \times 10^{-3}$ & & $5.8 \times 10^{-2}$ & $2.1 \times 10^{0}$ & $2.1 \times 10^{0}$ \\
\hline 1964 & 580 & & 731 & $3.1 \times 10^{0}$ & $1.1 \times 10^{-2}$ & & $2.5 \times 10^{-3}$ & & $1.9 \times 10^{-2}$ & $7.0 \times 10^{-1}$ & $7.2 \times 10^{-4}$ \\
\hline 1965 & 362 & 800 & 456 & $1.1 \times 10^{1}$ & $6.3 \times 10^{-2}$ & $8.0 \times 10^{-3}$ & $1.4 \times 10^{-2}$ & $2.1 \times 10^{-1}$ & $1.1 \times 10^{-1}$ & $3.9 \times 10^{0}$ & $4.2 \times 10^{0}$ \\
\hline 1966 & 316 & 520 & 399 & $5.1 \times 10^{0}$ & $3.4 \times 10^{-2}$ & $5.8 \times 10^{-3}$ & $7.5 \times 10^{-3}$ & $1.5 \times 10^{-1}$ & $5.7 \times 10^{-2}$ & $2.1 \times 10^{0}$ & $2.3 \times 10^{0}$ \\
\hline 1967 & 299 & 625 & 377 & $1.7 \times 10^{0}$ & $1.2 \times 10^{-2}$ & $1.6 \times 10^{-3}$ & $2.7 \times 10^{-3}$ & $4.1 \times 10^{-2}$ & $2.0 \times 10^{-2}$ & $7.4 \times 10^{-1}$ & $8.0 \times 10^{-1}$ \\
\hline 1968 & 273 & 470 & 343 & $1.8 \times 10^{0}$ & $1.4 \times 10^{-2}$ & $2.3 \times 10^{-3}$ & $3.2 \times 10^{-3}$ & $6.0 \times 10^{-2}$ & $2.4 \times 10^{-2}$ & $8.8 \times 10^{-1}$ & $9.6 \times 10^{-1}$ \\
\hline 1969 & 310 & 624 & 390 & $2.1 \times 10^{-1}$ & $1.4 \times 10^{-3}$ & $2.0 \times 10^{-4}$ & $3.2 \times 10^{-4}$ & $5.1 \times 10^{-3}$ & $2.4 \times 10^{-3}$ & $8.8 \times 10^{-2}$ & $9.6 \times 10^{-2}$ \\
\hline 1970 & 232 & 772 & 293 & $1.7 \times 10^{-1}$ & $1.6 \times 10^{-3}$ & $1.3 \times 10^{-4}$ & $3.5 \times 10^{-4}$ & $3.4 \times 10^{-3}$ & $2.7 \times 10^{-3}$ & $9.7 \times 10^{-2}$ & $1.0 \times 10^{-1}$ \\
\hline 1971 & 303 & 1245 & 381 & $4.6 \times 10^{0}$ & $3.2 \times 10^{-2}$ & $2.2 \times 10^{-3}$ & $7.2 \times 10^{-3}$ & $5.7 \times 10^{-2}$ & $5.5 \times 10^{-2}$ & $2.0 \times 10^{0}$ & $2.1 \times 10^{0}$ \\
\hline 1972 & 318 & 775 & 401 & $1.8 \times 10^{-1}$ & $1.2 \times 10^{-3}$ & $1.4 \times 10^{-4}$ & $2.7 \times 10^{-4}$ & $3.6 \times 10^{-3}$ & $2.0 \times 10^{-3}$ & $7.4 \times 10^{-2}$ & $8.0 \times 10^{-2}$ \\
\hline 1973 & 409 & 656 & 468 & $2.7 \times 10^{-2}$ & $1.4 \times 10^{-4}$ & $2.5 \times 10^{-5}$ & $3.5 \times 10^{-5}$ & $6.4 \times 10^{-4}$ & $2.7 \times 10^{-4}$ & $8.7 \times 10^{-3}$ & $9.6 \times 10^{-3}$ \\
\hline 1974 & 314 & 640 & 373 & $1.0 \times 10^{-3}$ & $6.8 \times 10^{-6}$ & $9.3 \times 10^{-7}$ & $1.6 \times 10^{-6}$ & $2.4 \times 10^{-5}$ & $1.2 \times 10^{-5}$ & $4.1 \times 10^{-4}$ & $4.5 \times 10^{-4}$ \\
\hline 1975 & 436 & 877 & 538 & $9.0 \times 10^{-3}$ & $4.4 \times 10^{-5}$ & $6.1 \times 10^{-6}$ & $9.9 \times 10^{-6}$ & $1.6 \times 10^{-4}$ & $7.6 \times 10^{-5}$ & $2.7 \times 10^{-3}$ & $2.9 \times 10^{-3}$ \\
\hline 1976 & 394 & 996 & 472 & $2.0 \times 10^{-3}$ & $1.1 \times 10^{-5}$ & $1.2 \times 10^{-6}$ & $2.5 \times 10^{-6}$ & $3.1 \times 10^{-5}$ & $1.9 \times 10^{-5}$ & $6.6 \times 10^{-4}$ & $7.1 \times 10^{-4}$ \\
\hline 1977 & 330 & 562 & 433 & $1.0 \times 10^{-1}$ & $6.5 \times 10^{-4}$ & $1.1 \times 10^{-4}$ & $1.4 \times 10^{-4}$ & $2.7 \times 10^{-3}$ & $1.1 \times 10^{-3}$ & $4.0 \times 10^{-2}$ & $4.4 \times 10^{-2}$ \\
\hline 1978 & 298 & 689 & 324 & $3.9 \times 10^{-4}$ & $2.8 \times 10^{-6}$ & $3.4 \times 10^{-7}$ & $7.2 \times 10^{-7}$ & $8.7 \times 10^{-6}$ & $5.5 \times 10^{-6}$ & $1.7 \times 10^{-4}$ & $1.8 \times 10^{-4}$ \\
\hline 1979 & 375 & 989 & 495 & $4.1 \times 10^{-1}$ & $2.3 \times 10^{-3}$ & $2.5 \times 10^{-4}$ & $4.9 \times 10^{-4}$ & $6.3 \times 10^{-3}$ & $3.8 \times 10^{-3}$ & $1.4 \times 10^{-1}$ & $1.5 \times 10^{-1}$ \\
\hline 1980 & 374 & 863 & 488 & $1.6 \times 10^{-3}$ & $9.0 \times 10^{-6}$ & $1.1 \times 10^{-6}$ & $1.9 \times 10^{-6}$ & $2.8 \times 10^{-5}$ & $1.5 \times 10^{-5}$ & $5.5 \times 10^{-4}$ & $5.9 \times 10^{-4}$ \\
\hline 1981 & 187 & 331 & 243 & $2.4 \times 10^{-4}$ & $2.7 \times 10^{-6}$ & $4.3 \times 10^{-7}$ & $5.9 \times 10^{-7}$ & $1.1 \times 10^{-5}$ & $4.5 \times 10^{-6}$ & $1.7 \times 10^{-4}$ & $1.8 \times 10^{-4}$ \\
\hline 1982 & 203 & 555 & 287 & $1.1 \times 10^{-4}$ & $1.2 \times 10^{-6}$ & $1.2 \times 10^{-7}$ & $2.3 \times 10^{-7}$ & $3.0 \times 10^{-6}$ & $1.7 \times 10^{-6}$ & $7.1 \times 10^{-5}$ & $7.5 \times 10^{-5}$ \\
\hline 1983 & 350 & 361 & 417 & $1.9 \times 10^{-3}$ & $1.2 \times 10^{-5}$ & $3.1 \times 10^{-6}$ & $2.7 \times 10^{-6}$ & $8.0 \times 10^{-5}$ & $2.1 \times 10^{-5}$ & $7.1 \times 10^{-4}$ & $8.1 \times 10^{-4}$ \\
\hline 1984 & 361 & 747 & 487 & $3.1 \times 10^{-4}$ & $1.8 \times 10^{-6}$ & $2.5 \times 10^{-7}$ & $3.8 \times 10^{-7}$ & $6.3 \times 10^{-6}$ & $2.9 \times 10^{-6}$ & $1.1 \times 10^{-4}$ & $1.2 \times 10^{-4}$ \\
\hline 1985 & 203 & 263 & 254 & & & & & & & & \\
\hline 1986 & 175 & 235 & 213 & & & & & & & & \\
\hline 1987 & 254 & 374 & 357 & & & & & & & & \\
\hline 1988 & 152 & 211 & 203 & & & & & & & & \\
\hline 1989 & 226 & 240 & 245 & & & & & & & & \\
\hline 1990 & 336 & 447 & 367 & & & & & & & & \\
\hline 1991 & 328 & 550 & 453 & & & & & & & & \\
\hline 1992 & 331 & 350 & 279 & & & & & & & & \\
\hline 1993 & 419 & 566 & 416 & & & & & & & & \\
\hline 1994 & 347 & 421 & 348 & & & & & & & & \\
\hline 1995 & 361 & 438 & 369 & $2.3 \times 10^{-3}$ & $1.4 \times 10^{-5}$ & $3.1 \times 10^{-6}$ & $3.7 \times 10^{-6}$ & $8.0 \times 10^{-5}$ & $2.8 \times 10^{-5}$ & $8.3 \times 10^{-4}$ & $9.4 \times 10^{-4}$ \\
\hline 1996 & 325 & 310 & 287 & & & & & & & & \\
\hline Total & & & & $6.6 \times 10^{1}$ & $4.4 \times 10^{-1}$ & $2.1 \times 10^{-2}$ & $9.8 \times 10^{-2}$ & $5.4 \times 10^{-1}$ & $7.5 \times 10^{-1}$ & $2.7 \times 10^{1}$ & $2.8 \times 10^{1}$ \\
\hline
\end{tabular}


Table 4-5. Liquid Zn-65 Releases and Dose

\begin{tabular}{|c|c|c|c|c|c|c|c|c|c|c|c|}
\hline Year & $\begin{array}{c}\text { River } \\
\text { Flow } \\
\text { Rate } \\
\left(\mathrm{m}^{3} / \mathrm{s}\right)\end{array}$ & $\begin{array}{c}\text { BJ } \\
\text { Derived } \\
\text { Flow } \\
\text { Rate } \\
\left(\mathrm{m}^{3} / \mathrm{s}\right)\end{array}$ & \begin{tabular}{|c|} 
PW \\
Derived \\
Flow \\
Rate \\
$\left(\mathrm{m}^{3} / \mathrm{s}\right)$
\end{tabular} & $\begin{array}{c}\text { Releases } \\
\text { to } \\
\text { Streams } \\
\text { (Ci) }\end{array}$ & $\begin{array}{l}\text { Max } \\
\text { Ind } \\
\text { Below } \\
\text { SRS } \\
\text { (mrem) }\end{array}$ & $\begin{array}{c}\text { Max } \\
\text { Ind } \\
\text { BJ } \\
\text { (mrem) }\end{array}$ & $\begin{array}{c}\text { Max } \\
\text { Ind } \\
\text { PW } \\
\text { (mrem) }\end{array}$ & $\begin{array}{c}\text { Pop } \\
\text { Dose } \\
\text { BJ } \\
\text { (per-rem) }\end{array}$ & $\begin{array}{c}\text { Pop } \\
\text { Dose } \\
\text { PW } \\
\text { (per-rem) }\end{array}$ & $\begin{array}{c}\text { Pop } \\
\text { Dose } \\
80 \mathrm{~km} \\
\text { (per-rem) }\end{array}$ & $\begin{array}{c}\text { Pop } \\
\text { Dose } \\
\text { Total } \\
\text { (per-rem) }\end{array}$ \\
\hline 1954 & 209 & & 263 & & & & & & & & \\
\hline 1955 & 169 & & 213 & & & & & & & & \\
\hline 1956 & 179 & & 225 & & & & & & & & \\
\hline 1957 & 235 & & 297 & & & & & & & & \\
\hline 1958 & 313 & & 394 & & & & & & & & \\
\hline 1959 & 276 & & 348 & & & & & & & & \\
\hline 1960 & 371 & & 468 & $4.3 \times 10^{0}$ & $2.0 \times 10^{-1}$ & & $2.8 \times 10^{-3}$ & & $2.2 \times 10^{-2}$ & $2.9 \times 10^{1}$ & $2.9 \times 10^{1}$ \\
\hline 1961 & 309 & & 389 & $2.1 \times 10^{1}$ & $1.1 \times 10^{0}$ & & $1.6 \times 10^{-2}$ & & $1.3 \times 10^{-1}$ & $1.7 \times 10^{2}$ & $1.7 \times 10^{2}$ \\
\hline 1962 & 300 & & 377 & $3.2 \times 10^{1}$ & $1.8 \times 10^{0}$ & & $2.6 \times 10^{-2}$ & & $2.1 \times 10^{-1}$ & $2.7 \times 10^{2}$ & $2.7 \times 10^{2}$ \\
\hline 1963 & 315 & & 397 & $3.4 \times 10^{1}$ & $1.8 \times 10^{0}$ & & $2.7 \times 10^{-2}$ & & $2.1 \times 10^{-1}$ & $2.7 \times 10^{2}$ & $2.7 \times 10^{2}$ \\
\hline 1964 & 580 & & 731 & $2.0 \times 10^{1}$ & $5.8 \times 10^{-1}$ & & $8.5 \times 10^{-3}$ & & $6.6 \times 10^{-2}$ & $8.7 \times 10^{1}$ & $8.7 \times 10^{1}$ \\
\hline 1965 & 362 & 800 & 456 & $8.9 \times 10^{0}$ & $4.2 \times 10^{-1}$ & $3.5 \times 10^{-3}$ & $6.1 \times 10^{-3}$ & $9.1 \times 10^{-2}$ & $4.8 \times 10^{-2}$ & $6.3 \times 10^{1}$ & $6.3 \times 10^{1}$ \\
\hline 1966 & 316 & 520 & 399 & $7.6 \times 10^{0}$ & $4.1 \times 10^{-1}$ & $4.5 \times 10^{-3}$ & $5.9 \times 10^{-3}$ & $1.2 \times 10^{-1}$ & $4.6 \times 10^{-2}$ & $6.1 \times 10^{1}$ & $6.1 \times 10^{1}$ \\
\hline 1967 & 299 & 625 & 377 & $7.3 \times 10^{0}$ & $4.1 \times 10^{-1}$ & $3.6 \times 10^{-3}$ & $6.0 \times 10^{-3}$ & $9.6 \times 10^{-2}$ & $4.7 \times 10^{-2}$ & $6.2 \times 10^{1}$ & $6.2 \times 10^{1}$ \\
\hline 1968 & 273 & 470 & 343 & $4.5 \times 10^{0}$ & $2.8 \times 10^{-1}$ & $3.0 \times 10^{-3}$ & $4.1 \times 10^{-3}$ & $7.8 \times 10^{-2}$ & $3.2 \times 10^{-2}$ & $4.2 \times 10^{1}$ & $4.2 \times 10^{9}$ \\
\hline 1969 & 310 & 624 & 390 & $1.5 \times 10^{0}$ & $8.4 \times 10^{-2}$ & $7.6 \times 10^{-4}$ & $1.2 \times 10^{-3}$ & $2.0 \times 10^{-2}$ & $9.5 \times 10^{-3}$ & $1.3 \times 10^{1}$ & $1.3 \times 10^{1}$ \\
\hline 1970 & 232 & 772 & 293 & $5.1 \times 10^{-1}$ & $3.7 \times 10^{-2}$ & $2.1 \times 10^{-4}$ & $5.4 \times 10^{-4}$ & $5.4 \times 10^{-3}$ & $4.2 \times 10^{-3}$ & $5.6 \times 10^{0}$ & $5.6 \times 10^{0}$ \\
\hline 1971 & 303 & 1245 & 381 & $1.8 \times 10^{0}$ & $9.8 \times 10^{-2}$ & $4.4 \times 10^{-4}$ & $1.4 \times 10^{-3}$ & $1.2 \times 10^{-2}$ & $1.1 \times 10^{-2}$ & $1.5 \times 10^{1}$ & $1.5 \times 10^{1}$ \\
\hline 1972 & 318 & 775 & 401 & & & & & & & & \\
\hline 1973 & 409 & 656 & 468 & & & & & & & & \\
\hline 1974 & 314 & 640 & 373 & $1.4 \times 10^{-1}$ & $7.6 \times 10^{-3}$ & $6.8 \times 10^{-5}$ & $1.2 \times 10^{-4}$ & $1.8 \times 10^{-3}$ & $9.1 \times 10^{-4}$ & $1.1 \times 10^{0}$ & $1.1 \times 10^{0}$ \\
\hline 1975 & 436 & 877 & 538 & $2.0 \times 10^{-3}$ & $7.8 \times 10^{-5}$ & $7.1 \times 10^{-7}$ & $1.2 \times 10^{-6}$ & $1.9 \times 10^{-5}$ & $9.0 \times 10^{-6}$ & $1.2 \times 10^{-2}$ & $1.2 \times 10^{-2}$ \\
\hline 1976 & 394 & 996 & 472 & & & & & & & & \\
\hline 1977 & 330 & 562 & 433 & & & & & & & & \\
\hline 1978 & 298 & 689 & 324 & & & & & & & & \\
\hline 1979 & 375 & 989 & 495 & & & & & & & & \\
\hline 1980 & 374 & 863 & 488 & & & & & & & & \\
\hline 1981 & .187 & 331 & 243 & & & & & & & & \\
\hline 1982 & 203 & 555 & 287 & & & & & & & & \\
\hline 1983 & 350 & 361 & 417 & & & & & & & & \\
\hline 1984 & 361 & 747 & 487 & & & & & & & & \\
\hline 1985 & 203 & 263 & 254 & & & & & & & & \\
\hline 1986 & 175 & 235 & 213 & & & & & & & & \\
\hline 1987 & 254 & 374 & 357 & & & & & & & & \\
\hline 1988 & 152 & 211 & 203 & & & & & & & & \\
\hline 1989 & 226 & 240 & 245 & & & & & & & & \\
\hline 1990 & 336 & 447 & 367 & & & & & & & & \\
\hline 1991 & 328 & 550 & 453 & & & & & & & & \\
\hline 1992 & 331 & 350 & 279 & & & & & & & & \\
\hline 1993 & 419 & 566 & 416 & & & & & & & & \\
\hline 1994 & 347 & 421 & 348 & & & & & & & . & \\
\hline 1995 & 361 & 438 & 369 & & & & & & & & \\
\hline 1996 & 325 & 310 & 287 & & & & & & & & \\
\hline Total & & & & $1.4 \times 10^{2}$ & $7.3 \times 10^{0}$ & $1.6 \times 10^{-2}$ & $1.1 \times 10^{-1}$ & $4.2 \times 10^{-1}$ & $8.3 \times 10^{-1}$ & $1.1 \times 10^{3}$ & $1.1 \times 10^{3}$ \\
\hline
\end{tabular}


Chapter 4. Releases and Doses

Table 4-6. Atmospheric C-14 Releases and Dose

\begin{tabular}{|c|c|c|c|}
\hline Year & $\begin{array}{c}\text { Release } \\
\text { (Ci) }\end{array}$ & $\begin{array}{l}\text { Maximum } \\
\text { Individual } \\
\text { Dose } \\
\text { at Boundary } \\
\text { (mrem) }\end{array}$ & $\begin{array}{c}\text { Population } \\
\text { Dose } \\
\text { (per-rem) }\end{array}$ \\
\hline \multicolumn{4}{|l|}{1954} \\
\hline 1955 & $8.3 \times 10^{1}$ & $2.7 \times 10^{-2}$ & $8.3 \times 10^{-1}$ \\
\hline 1956 & $8.4 \times 10^{1}$ & $2.8 \times 10^{-2}$ & $8.4 \times 10^{-1}$ \\
\hline 1957 & $8.4 \times 10^{1}$ & $2.8 \times 10^{-2}$ & $8.4 \times 10^{-1}$ \\
\hline 1958 & $8.4 \times 10^{1}$ & $2.8 \times 10^{-2}$ & $8.4 \times 10^{-1}$ \\
\hline 1959 & $1.4 \times 10^{2}$ & $4.6 \times 10^{-2}$ & $1.4 \times 10^{0}$ \\
\hline 1960 & $1.4 \times 10^{2}$ & $4.6 \times 10^{-2}$ & $1.4 \times 10^{0}$ \\
\hline 1961 & $1.4 \times 10^{2}$ & $4.6 \times 10^{-2}$ & $1.4 \times 10^{0}$ \\
\hline 1962 & $1.4 \times 10^{2}$ & $4.6 \times 10^{-2}$ & $1.4 \times 10^{0}$ \\
\hline 1963 & $1.4 \times 10^{2}$ & $4.6 \times 10^{-2}$ & $1.4 \times 10^{0}$ \\
\hline 1964 & $1.1 \times 10^{2}$ & $3.6 \times 10^{-2}$ & $1.1 \times 10^{0}$ \\
\hline 1965 & $\quad 1.1 \times 10^{2}$ & $3.6 \times 10^{-2}$ & $1.1 \times 10^{0}$ \\
\hline 1966 & $1.1 \times 10^{2}$ & $3.6 \times 10^{-2}$ & $1.1 \times 10^{0}$ \\
\hline 1967 & $1.1 \times 10^{2}$ & $3.6 \times 10^{-2}$ & $1.1 \times 10^{0}$ \\
\hline 1968 & $8.7 \times 10^{1}$ & $2.9 \times 10^{-2}$ & $8.7 \times 10^{-1}$ \\
\hline 1969 & $8.7 \times 10^{1}$ & $2.9 \times 10^{-2}$ & $8.7 \times 10^{-1}$ \\
\hline 1970 & $8.8 \times 10^{1}$ & $2.9 \times 10^{-2}$ & $8.8 \times 10^{-1}$ \\
\hline 1971 & $8.7 \times 10^{1}$ & $2.9 \times 10^{-2}$ & $8.7 \times 10^{-1}$ \\
\hline 1972 & $8.7 \times 10^{1}$ & $2.9 \times 10^{-2}$ & $8.7 \times 10^{-1}$ \\
\hline 1973 & $8.7 \times 10^{1}$ & $2.9 \times 10^{-2}$ & $8.7 \times 10^{-1}$ \\
\hline 1974 & $8.0 \times 10^{1}$ & $2.6 \times 10^{-2}$ & $8.0 \times 10^{-1}$ \\
\hline 1975 & $6.6 \times 10^{1}$ & $2.2 \times 10^{-2}$ & $6.6 \times 10^{-1}$ \\
\hline 1976 & $6.9 \times 10^{1}$ & $2.3 \times 10^{-2}$ & $6.9 \times 10^{-1}$ \\
\hline 1977 & $6.3 \times 10^{1}$ & $2.1 \times 10^{-2}$ & $6.3 \times 10^{-1}$ \\
\hline 1978 & $5.7 \times 10^{1}$ & $1.9 \times 10^{-2}$ & $5.7 \times 10^{-1}$ \\
\hline 1979 & $5.6 \times 10^{1}$ & $1.8 \times 10^{-2}$ & $5.6 \times 10^{-1}$ \\
\hline 1980 & $6.6 \times 10^{1}$ & $2.2 \times 10^{-2}$ & $6.6 \times 10^{-1}$ \\
\hline 1981 & $6.9 \times 10^{1}$ & $2.3 \times 10^{-2}$ & $6.9 \times 10^{-1}$ \\
\hline 1982 & $8.0 \times 10^{1}$ & $2.6 \times 10^{-2}$ & $8.0 \times 10^{-1}$ \\
\hline 1983 & $9.0 \times 10^{1}$ & $3.0 \times 10^{-2}$ & $9.0 \times 10^{-1}$ \\
\hline 1984 & $8.3 \times 10^{1}$ & $2.7 \times 10^{-2}$ & $8.3 \times 10^{-1}$ \\
\hline 1985 & $7.6 \times 10^{1}$ & $2.5 \times 10^{-2}$ & $7.6 \times 10^{-1}$ \\
\hline 1986 & $4.6 \times 10^{1}$ & $1.5 \times 10^{-2}$ & $4.6 \times 10^{-1}$ \\
\hline 1987 & $4.1 \times 10^{1}$ & $1.4 \times 10^{-2}$ & $4.1 \times 10^{-1}$ \\
\hline 1988 & $2.4 \times 10^{1}$ & $7.9 \times 10^{-3}$ & $2.4 \times 10^{-1}$ \\
\hline 1989 & $1.8 \times 10^{1}$ & $5.9 \times 10^{-3}$ & $1.8 \times 10^{-1}$ \\
\hline 1990 & $6.7 \times 10^{-1}$ & $2.2 \times 10^{-4}$ & $6.7 \times 10^{-3}$ \\
\hline 1991 & $6.2 \times 10^{-1}$ & $2.0 \times 10^{-4}$ & $6.2 \times 10^{-3}$ \\
\hline 1992 & $1.9 \times 10^{-1}$ & $6.1 \times 10^{-5}$ & $1.9 \times 10^{-3}$ \\
\hline 1993 & $1.7 \times 10^{-2}$ & $5.6 \times 10^{-6}$ & $1.7 \times 10^{-4}$ \\
\hline 1994 & $3.7 \times 10^{-2}$ & $1.2 \times 10^{-5}$ & $3.7 \times 10^{-4}$ \\
\hline \multicolumn{4}{|l|}{1995} \\
\hline 1996 & $8.1 \times 10^{0}$ & $2.7 \times 10^{-3}$ & $8.1 \times 10^{-2}$ \\
\hline Total & $3.0 \times 10^{3}$ & $9.8 \times 10^{-1}$ & $3.0 \times 10^{1}$ \\
\hline
\end{tabular}


Table 4-7. Atmospheric Cs-137 Releases and Dose

\begin{tabular}{|c|c|c|c|}
\hline Year & $\begin{array}{l}\text { Release } \\
\text { (Ci) }\end{array}$ & $\begin{array}{l}\text { Maximum Individual } \\
\text { Dose at Boundary } \\
\text { (mrem) }\end{array}$ & $\begin{array}{c}\text { Population } \\
\text { Dose } \\
\text { (per-rem) }\end{array}$ \\
\hline \multicolumn{4}{|l|}{1954} \\
\hline 1955 & $1.4 \times 10^{0}$ & $1.8 \times 10^{-1}$ & $1.3 \times 10^{1}$ \\
\hline 1956 & $2.4 \times 10^{-1}$ & $3.2 \times 10^{-2}$ & $2.3 \times 10^{0}$ \\
\hline 1957 & $6.3 \times 10^{-2}$ & $8.5 \times 10^{-3}$ & $6.1 \times 10^{-1}$ \\
\hline 1958 & $2.6 \times 10^{-2}$ & $3.5 \times 10^{-3}$ & $2.5 \times 10^{-1}$ \\
\hline 1959 & $1.4 \times 10^{-1}$ & $1.9 \times 10^{-2}$ & $1.3 \times 10^{0}$ \\
\hline 1960 & $1.2 \times 10^{-1}$ & $1.7 \times 10^{-2}$ & $1.2 \times 10^{0}$ \\
\hline 1961 & $2.5 \times 10^{-2}$ & $3.3 \times 10^{-3}$ & $2.4 \times 10^{-1}$ \\
\hline 1962 & $3.7 \times 10^{-2}$ & $5.0 \times 10^{-3}$ & $3.6 \times 10^{-1}$ \\
\hline 1963 & $2.5 \times 10^{-2}$ & $3.4 \times 10^{-3}$ & $2.4 \times 10^{-1}$ \\
\hline 1964 & $6.7 \times 10^{-2}$ & $9.0 \times 10^{-3}$ & $6.4 \times 10^{-1}$ \\
\hline 1965 & $1.8 \times 10^{-2}$ & $2.4 \times 10^{-3}$ & $1.7 \times 10^{-1}$ \\
\hline 1966 & $4.8 \times 10^{-2}$ & $6.5 \times 10^{-3}$ & $4.7 \times 10^{-1}$ \\
\hline 1967 & $1.8 \times 10^{-2}$ & $2.4 \times 10^{-3}$ & $1.7 \times 10^{-1}$ \\
\hline 1968 & $4.0 \times 10^{-2}$ & $5.4 \times 10^{-3}$ & $3.9 \times 10^{-1}$ \\
\hline 1969 & $8.5 \times 10^{-2}$ & $1.2 \times 10^{-2}$ & $8.2 \times 10^{-1}$ \\
\hline 1970 & $4.2 \times 10^{-2}$ & $5.6 \times 10^{-3}$ & $4.0 \times 10^{-1}$ \\
\hline 1971 & $9.3 \times 10^{-3}$ & $1.3 \times 10^{-3}$ & $9.0 \times 10^{-2}$ \\
\hline 1972 & $2.4 \times 10^{-3}$ & $3.3 \times 10^{-4}$ & $2.4 \times 10^{-2}$ \\
\hline 1973 & $2.6 \times 10^{-3}$ & $3.5 \times 10^{-4}$ & $2.5 \times 10^{-2}$ \\
\hline 1974 & $1.3 \times 10^{-3}$ & $1.8 \times 10^{-4}$ & $1.3 \times 10^{-2}$ \\
\hline 1975 & $1.1 \times 10^{-3}$ & $1.4 \times 10^{-4}$ & $1.0 \times 10^{-2}$ \\
\hline 1976 & $1.3 \times 10^{-3}$ & $1.8 \times 10^{-4}$ & $1.3 \times 10^{-2}$ \\
\hline 1977 & $1.9 \times 10^{-3}$ & $2.5 \times 10^{-4}$ & $1.8 \times 10^{-2}$ \\
\hline 1978 & $2.0 \times 10^{-3}$ & $2.8 \times 10^{-4}$ & $2.0 \times 10^{-2}$ \\
\hline 1979 & $5.4 \times 10^{-3}$ & $7.2 \times 10^{-4}$ & $5.2 \times 10^{-2}$ \\
\hline 1980 & $2.6 \times 10^{-3}$ & $3.6 \times 10^{-4}$ & $2.5 \times 10^{-2}$ \\
\hline 1981 & $3.1 \times 10^{-3}$ & $4.1 \times 10^{-4}$ & $3.0 \times 10^{-2}$ \\
\hline 1982 & $9.6 \times 10^{-4}$ & $1.3 \times 10^{4}$ & $9.3 \times 10^{-3}$ \\
\hline 1983 & $9.9 \times 10^{-4}$ & $1.3 \times 10^{-4}$ & $9.6 \times 10^{-3}$ \\
\hline 1984 & $1.9 \times 10^{-3}$ & $2.6 \times 10^{-4}$ & $1.9 \times 10^{-2}$ \\
\hline 1985 & $5.2 \times 10^{-3}$ & $7.0 \times 10^{-4}$ & $5.0 \times 10^{-2}$ \\
\hline 1986 & $3.0 \times 10^{-3}$ & $4.0 \times 10^{-4}$ & $2.8 \times 10^{-2}$ \\
\hline 1987 & $1.1 \times 10^{0}$ & $1.4 \times 10^{-1}$ & $1.0 \times 10^{1}$ \\
\hline 1988 & $1.8 \times 10^{-3}$ & $2.4 \times 10^{-4}$ & $1.7 \times 10^{-2}$ \\
\hline 1989 & $9.6 \times 10^{-4}$ & $1.3 \times 10^{-4}$ & $9.2 \times 10^{-3}$ \\
\hline 1990 & $2.1 \times 10^{-3}$ & $2.9 \times 10^{-4}$ & $2.1 \times 10^{-2}$ \\
\hline 1991 & $3.3 \times 10^{-4}$ & $4.5 \times 10^{-5}$ & $3.2 \times 10^{-3}$ \\
\hline 1992 & $2.5 \times 10^{-4}$ & $3.4 \times 10^{-5}$ & $2.4 \times 10^{-3}$ \\
\hline 1993 & $6.3 \times 10^{-4}$ & $8.6 \times 10^{-5}$ & $6.1 \times 10^{-3}$ \\
\hline 1994 & $1.6 \times 10^{-4}$ & $2.1 \times 10^{-5}$ & $1.5 \times 10^{-3}$ \\
\hline 1995 & $1.5 \times 10^{-2}$ & $2.0 \times 10^{-3}$ & $1.4 \times 10^{-1}$ \\
\hline 1996 & $4.8 \times 10^{-3}$ & $6.5 \times 10^{-4}$ & $4.7 \times 10^{-2}$ \\
\hline Total & $3.5 \times 10^{0}$ & $4.7 \times 10^{-1}$ & $3.4 \times 10^{1}$ \\
\hline
\end{tabular}


Table 4-8. Liquid Cs-137 Releases and Dose

\begin{tabular}{|c|c|c|c|c|c|c|c|c|c|c|c|}
\hline Year & $\begin{array}{c}\text { River } \\
\text { Flow } \\
\text { Rate } \\
\left(\mathrm{m}^{3} / \mathrm{s}\right)\end{array}$ & $\begin{array}{c}\mathrm{BJ} \\
\text { Derived } \\
\text { Flow } \\
\text { Rate } \\
\left(\mathrm{m}^{3} / \mathrm{s}\right)\end{array}$ & \begin{tabular}{|c|} 
PW \\
Derived \\
Flow \\
Rate \\
$\left(\mathrm{m}^{3} / \mathrm{s}\right)$
\end{tabular} & $\begin{array}{c}\text { Adjusted } \\
\text { Releases } \\
\text { to } \\
\text { Streams } \\
\text { (Ci) }\end{array}$ & $\begin{array}{c}\text { Max } \\
\text { Ind } \\
\text { Below } \\
\text { SRS } \\
\text { (mrem) }\end{array}$ & $\begin{array}{c}\text { Max } \\
\text { Ind } \\
\text { BJ } \\
\text { (mrem) }\end{array}$ & $\begin{array}{c}\text { Max } \\
\text { Ind } \\
\text { PW } \\
\text { (mrem) }\end{array}$ & $\begin{array}{c}\text { Pop } \\
\text { Dose } \\
\text { BJ } \\
\text { (mrem) }\end{array}$ & $\begin{array}{c}\text { Pop } \\
\text { Dose } \\
\text { PW } \\
\text { (per-rem) }\end{array}$ & $\begin{array}{c}\text { Pop } \\
\text { Dose } \\
80 \mathrm{~km} \\
\text { (per-rem) }\end{array}$ & $\begin{array}{c}\text { Pop } \\
\text { Dose } \\
\text { Total } \\
\text { (per-rem) }\end{array}$ \\
\hline 1954 & 209 & & 263 & & & & & & & & \\
\hline 1955 & 169 & & 213 & $2.4 \times 10^{-1}$ & $1.3 \times 10^{-1}$ & & $1.3 \times 10^{-3}$ & & $9.7 \times 10^{-3}$ & $2.6 \times 10^{-1}$ & $2.7 \times 10^{-1}$ \\
\hline 1956 & 179 & & 225 & $6.4 \times 10^{-1}$ & $3.3 \times 10^{-1}$ & & $3.3 \times 10^{-3}$ & & $2.5 \times 10^{-2}$ & $6.5 \times 10^{-1}$ & $6.8 \times 10^{-1}$ \\
\hline 1957 & 235 & & 297 & $1.7 \times 10^{1}$ & $6.9 \times 10^{0}$ & & $6.8 \times 10^{-2}$ & & $5.1 \times 10^{-1}$ & $1.4 \times 10^{1}$ & $1.4 \times 10^{1}$ \\
\hline 1958 & 313 & & 394 & $1.1 \times 10^{0}$ & $3.4 \times 10^{-1}$ & & $3.3 \times 10^{-3}$ & & $2.5 \times 10^{-2}$ & $6.6 \times 10^{-1}$ & $6.9 \times 10^{-1}$ \\
\hline 1959 & 276 & & 348 & $2.8 \times 10^{0}$ & $9.4 \times 10^{-1}$ & & $9.3 \times 10^{-3}$ & & $7.0 \times 10^{-2}$ & $1.9 \times 10^{0}$ & $1.9 \times 10^{0}$ \\
\hline 1960 & 371 & & 468 & $1.2 \times 10^{1}$ & $3.1 \times 10^{0}$ & & $3.0 \times 10^{-2}$ & & $2.3 \times 10^{-1}$ & $6.0 \times 10^{0}$ & $6.3 \times 10^{0}$ \\
\hline 1961 & 309 & & 389 & $6.6 \times 10^{0}$ & $2.0 \times 10^{0}$ & & $2.0 \times 10^{-2}$ & & $1.5 \times 10^{-1}$ & $3.9 \times 10^{0}$ & $4.1 \times 10^{0}$ \\
\hline 1962 & 300 & & 377 & $1.3 \times 10^{1}$ & $4.1 \times 10^{0}$ & & $4.0 \times 10^{-2}$ & & $3.0 \times 10^{-1}$ & $8.0 \times 10^{0}$ & $8.3 \times 10^{0}$ \\
\hline 1963 & 315 & & 397 & $3.4 \times 10^{1}$ & $1.0 \times 10^{4}$ & & $1.0 \times 10^{-1}$ & & $7.5 \times 10^{-1}$ & $2.0 \times 10^{1}$ & $2.1 \times 10^{1}$ \\
\hline 1964 & 580 & & 731 & $3.8 \times 10^{4}$ & $6.1 \times 10^{0}$ & & $6.0 \times 10^{-2}$ & & $4.6 \times 10^{-1}$ & $1.2 \times 10^{1}$ & $1.3 \times 10^{1}$ \\
\hline 1965 & 362 & 800 & 456 & $1.2 \times 10^{1}$ & $3.0 \times 10^{0}$ & $1.7 \times 10^{-2}$ & $3.0 \times 10^{-2}$ & $4.2 \times 10^{-1}$ & $2.3 \times 10^{-1}$ & $6.0 \times 10^{0}$ & $6.6 \times 10^{0}$ \\
\hline 1966 & 316 & 520 & 399 & $1.3 \times 10^{1}$ & $3.8 \times 10^{0}$ & $2.9 \times 10^{-2}$ & $3.7 \times 10^{-2}$ & $7.0 \times 10^{-1}$ & $2.8 \times 10^{-1}$ & $7.5 \times 10^{0}$ & $8.4 \times 10^{0}$ \\
\hline 1967 & 299 & 625 & 377 & $2.8 \times 10^{1}$ & $8.6 \times 10^{0}$ & $5.1 \times 10^{-2}$ & $8.5 \times 10^{-2}$ & $1.2 \times 10^{0}$ & $6.4 \times 10^{-1}$ & $1.7 \times 10^{1}$ & $1.9 \times 10^{1}$ \\
\hline 1968 & 273 & 470 & 343 & $1.5 \times 10^{1}$ & $5.2 \times 10^{0}$ & $3.8 \times 10^{-2}$ & $5.1 \times 10^{-2}$ & $9.2 \times 10^{-1}$ & $3.9 \times 10^{-1}$ & $1.0 \times 10^{1}$ & $1.2 \times 10^{1}$ \\
\hline 1969 & 310 & 624 & 390 & $7.7 \times 10^{0}$ & $2.3 \times 10^{\circ}$ & $1.4 \times 10^{-2}$ & $2.3 \times 10^{-2}$ & $3.5 \times 10^{-1}$ & $1.7 \times 10^{-1}$ & $4.6 \times 10^{0}$ & $5.1 \times 10^{0}$ \\
\hline 1970 & 232 & 772 & 293 & $7.4 \times 10^{0}$ & $3.0 \times 10^{0}$ & $1.1 \times 10^{-2}$ & $2.9 \times 10^{-2}$ & $2.7 \times 10^{-1}$ & $2.2 \times 10^{-1}$ & $5.9 \times 10^{0}$ & $6.4 \times 10^{0}$ \\
\hline 1971 & 303 & 1245 & 381 & $1.1 \times 10^{0}$ & $3.3 \times 10^{-1}$ & $1.0 \times 10^{-3}$ & $3.3 \times 10^{-3}$ & $2.4 \times 10^{-2}$ & $2.5 \times 10^{-2}$ & $6.5 \times 10^{-1}$ & $7.0 \times 10^{-1}$ \\
\hline 1972 & 318 & 775 & 401 & $4.6 \times 10^{-1}$ & $1.3 \times 10^{-1}$ & $6.8 \times 10^{-4}$ & $1.3 \times 10^{-3}$ & $1.7 \times 10^{-2}$ & $1.0 \times 10^{-2}$ & $2.6 \times 10^{-1}$ & $2.9 \times 10^{-1}$ \\
\hline 1973 & 409 & 656 & 468 & $3.2 \times 10^{-1}$ & $7.3 \times 10^{-2}$ & $5.7 \times 10^{-4}$ & $8.0 \times 10^{-4}$ & $1.4 \times 10^{-2}$ & $6.0 \times 10^{-3}$ & $1.4 \times 10^{-1}$ & $1.6 \times 10^{-1}$ \\
\hline 1974 & 314 & 640 & 373 & $5.3 \times 10^{-1}$ & $1.6 \times 10^{-1}$ & $9.5 \times 10^{-4}$ & $1.6 \times 10^{-3}$ & $2.3 \times 10^{-2}$ & $1.2 \times 10^{-2}$ & $3.1 \times 10^{-1}$ & $3.4 \times 10^{-1}$ \\
\hline 1975 & 436 & 877 & 538 & $2.7 \times 10^{-1}$ & $5.7 \times 10^{-2}$ & $3.5 \times 10^{-4}$ & $5.7 \times 10^{-4}$ & $8.6 \times 10^{-3}$ & $4.3 \times 10^{-3}$ & $1.1 \times 10^{-1}$ & $1.3 \times 10^{-1}$ \\
\hline 1976 & 394 & 996 & 472 & $1.1 \times 10^{-1}$ & $2.5 \times 10^{-2}$ & $1.2 \times 10^{-4}$ & $2.6 \times 10^{-4}$ & $3.0 \times 10^{-3}$ & $2.0 \times 10^{-3}$ & $4.9 \times 10^{-2}$ & $5.4 \times 10^{-2}$ \\
\hline 1977 & 330 & 562 & 433 & $1.8 \times 10^{-1}$ & $5.0 \times 10^{-2}$ & $3.7 \times 10^{-4}$ & $4.8 \times 10^{-4}$ & $9.0 \times 10^{-3}$ & $3.6 \times 10^{-3}$ & $9.9 \times 10^{-2}$ & $1.1 \times 10^{-1}$ \\
\hline 1978 & 298 & 689 & 324 & $7.5 \times 10^{-2}$ & $2.3 \times 10^{-2}$ & $1.3 \times 10^{-4}$ & $2.7 \times 10^{-4}$ & $3.1 \times 10^{-3}$ & $2.0 \times 10^{-3}$ & $4.6 \times 10^{-2}$ & $5.1 \times 10^{-2}$ \\
\hline 1979 & 375 & 989 & 495 & $7.4 \times 10^{-2}$ & $1.8 \times 10^{-2}$ & $8.7 \times 10^{-5}$ & $1.7 \times 10^{-4}$ & $2.1 \times 10^{-3}$ & $1.3 \times 10^{-3}$ & $3.6 \times 10^{-2}$ & $4.0 \times 10^{-2}$ \\
\hline 1980 & 374 & 863 & 488 & $5.6 \times 10^{-2}$ & $1.4 \times 10^{-2}$ & $7.6 \times 10^{-5}$ & $1.3 \times 10^{-4}$ & $1.8 \times 10^{-3}$ & $1.0 \times 10^{-3}$ & $2.8 \times 10^{-2}$ & $3.0 \times 10^{-2}$ \\
\hline 1981 & 187 & 331 & 243 & $7.8 \times 10^{-2}$ & $3.9 \times 10^{-2}$ & $2.7 \times 10^{-4}$ & $3.7 \times 10^{-4}$ & $6.7 \times 10^{-3}$ & $2.8 \times 10^{-3}$ & $7.7 \times 10^{-2}$ & $8.6 \times 10^{-2}$ \\
\hline 1982 & 203 & 555 & 287 & $5.6 \times 10^{-2}$ & $2.6 \times 10^{-2}$ & $1.2 \times 10^{-4}$ & $2.3 \times 10^{-4}$ & $2.9 \times 10^{-3}$ & $1.7 \times 10^{-3}$ & $5.1 \times 10^{-2}$ & $5.6 \times 10^{-2}$ \\
\hline 1983 & $\overline{350}$ & 361 & 417 & $5.1 \times 10^{-2}$ & $1.4 \times 10^{-2}$ & $1.6 \times 10^{-4}$ & $1.4 \times 10^{-4}$ & $4.0 \times 10^{-3}$ & $1.1 \times 10^{-3}$ & $2.7 \times 10^{-2}$ & $3.2 \times 10^{-2}$ \\
\hline 1984 & 361 & 747 & 487 & $8.3 \times 10^{-2}$ & $2.2 \times 10^{-2}$ & $1.3 \times 10^{-4}$ & $2.0 \times 10^{-4}$ & $3.2 \times 10^{-3}$ & $1.5 \times 10^{-3}$ & $4.2 \times 10^{-2}$ & $4.7 \times 10^{-2}$ \\
\hline 1985 & 203 & 263 & 254 & $3.5 \times 10^{-2}$ & $1.6 \times 10^{-2}$ & $1.5 \times 10^{-4}$ & $1.6 \times 10^{-4}$ & $3.7 \times 10^{-3}$ & $1.2 \times 10^{-3}$ & $3.1 \times 10^{-2}$ & $3.6 \times 10^{-2}$ \\
\hline 1986 & 175 & 235 & 213 & $3.7 \times 10^{-2}$ & $2.0 \times 10^{-2}$ & $1.8 \times 10^{-4}$ & $2.0 \times 10^{-4}$ & $4.4 \times 10^{-3}$ & $1.5 \times 10^{-3}$ & $3.9 \times 10^{-2}$ & $4.5 \times 10^{-2}$ \\
\hline 1987 & 254 & 374 & 357 & $1.3 \times 10^{-1}$ & $4.9 \times 10^{-2}$ & $4.1 \times 10^{-4}$ & $4.3 \times 10^{-4}$ & $1.0 \times 10^{-2}$ & $3.3 \times 10^{-3}$ & $9.6 \times 10^{-2}$ & $1.1 \times 10^{-1}$ \\
\hline 1988 & 152 & 211 & 203 & $2.0 \times 10^{-1}$ & $1.2 \times 10^{-1}$ & $1.1 \times 10^{-3}$ & $1.1 \times 10^{-3}$ & $2.6 \times 10^{-2}$ & $8.5 \times 10^{-3}$ & $2.4 \times 10^{-1}$ & $2.7 \times 10^{-1}$ \\
\hline 1989 & 226 & 240 & 245 & $7.2 \times 10^{-2}$ & $3.0 \times 10^{-2}$ & $3.5 \times 10^{-4}$ & $3.4 \times 10^{-4}$ & $8.5 \times 10^{-3}$ & $2.6 \times 10^{-3}$ & $5.9 \times 10^{-2}$ & $7.0 \times 10^{-2}$ \\
\hline 1990 & 336 & 447 & 367 & $1.7 \times 10^{-2}$ & $4.7 \times 10^{-3}$ & $4.4 \times 10^{-5}$ & $5.4 \times 10^{-5}$ & $1.1 \times 10^{-3}$ & $4.1 \times 10^{-4}$ & $9.3 \times 10^{-3}$ & $1.1 \times 10^{-2}$ \\
\hline 1991 & 328 & 550 & 453 & $9.2 \times 10^{-3}$ & $2.6 \times 10^{-3}$ & $1.9 \times 10^{-5}$ & $2.4 \times 10^{-5}$ & $4.7 \times 10^{-4}$ & $1.8 \times 10^{-4}$ & $5.2 \times 10^{-3}$ & $5.8 \times 10^{-3}$ \\
\hline 1992 & 331 & 350 & 279 & $3.5 \times 10^{-2}$ & $9.9 \times 10^{-3}$ & $1.2 \times 10^{-4}$ & $1.5 \times 10^{-4}$ & $2.8 \times 10^{-3}$ & $1.1 \times 10^{-3}$ & $1.9 \times 10^{-2}$ & $2.3 \times 10^{-2}$ \\
\hline 1993 & 419 & 566 & 416 & $8.8 \times 10^{-2}$ & $2.0 \times 10^{-2}$ & $1.8 \times 10^{-4}$ & $2.5 \times 10^{-4}$ & $4.4 \times 10^{-3}$ & $1.9 \times 10^{-3}$ & $3.9 \times 10^{-2}$ & $4.5 \times 10^{-2}$ \\
\hline $1994^{a}$ & 347 & 421 & $\overline{348}$ & $2.0 \times 10^{-1}$ & $5.4 \times 10^{-2}$ & $5.5 \times 10^{-4}$ & $6.7 \times 10^{-4}$ & $1.3 \times 10^{-2}$ & $5.0 \times 10^{-3}$ & $1.1 \times 10^{-1}$ & $1.2 \times 10^{-1}$ \\
\hline $1995^{\mathrm{a}}$ & 361 & 438 & 369 & $2.0 \times 10^{-1}$ & $5.2 \times 10^{-2}$ & $5.3 \times 10^{-4}$ & $6.3 \times 10^{-4}$ & $1.3 \times 10^{-2}$ & $4.8 \times 10^{-3}$ & $1.0 \times 10^{-1}$ & $1.2 \times 10^{-1}$ \\
\hline $1996^{a}$ & 325 & 310 & 287 & $1.6 \times 10^{-1}$ & $4.6 \times 10^{-2}$ & $6.0 \times 10^{-4}$ & $6.5 \times 10^{-4}$ & $1.5 \times 10^{-2}$ & $4.9 \times 10^{-3}$ & $9.1 \times 10^{-2}$ & $1.1 \times 10^{-1}$ \\
\hline Total & & & & $2.1 \times 10^{2}$ & $6.1 \times 10^{1}$ & $1.7 \times 10^{-1}$ & $6.1 \times 10^{-1}$ & $4.1 \times 10^{0}$ & $4.6 \times 10^{\circ}$ & $1.2 \times 10^{2}$ & $1.3 \times 10^{2}$ \\
\hline
\end{tabular}


Table 4-9. Atmospheric lodine Releases and Dose

\begin{tabular}{|c|c|c|c|c|c|c|c|c|}
\hline \multirow[t]{2}{*}{ Year } & \multirow[t]{2}{*}{$\begin{array}{c}1-129 \\
\text { Release } \\
\text { (Ci) }\end{array}$} & \multirow[t]{2}{*}{$\begin{array}{c}\text { T-131 } \\
\text { Release } \\
\text { (Ci) }\end{array}$} & \multicolumn{3}{|c|}{$\begin{array}{l}\text { Maximum Individual } \\
\text { Dose at Boundary } \\
\text { (mrem) }\end{array}$} & \multicolumn{3}{|c|}{$\begin{array}{l}\text { Population Dose } \\
\text { (per-rem) }\end{array}$} \\
\hline & & & T-129 & $|-13|$ & Totall & $T-129$ & $|-13|$ & Total \\
\hline \multicolumn{9}{|l|}{1954} \\
\hline 1955 & $2.1 \times 10^{-1}$ & $6.9 \times 10^{1}$ & $1.4 \times 10^{-1}$ & $5.8 \times 10^{-1}$ & $7.2 \times 10^{-1}$ & $3.8 \times 10^{0}$ & $2.3 \times 10^{1}$ & $2.7 \times 10^{1}$ \\
\hline 1956 & $2.1 \times 10^{-1}$ & $1.6 \times 10^{3}$ & $1.4 \times 10^{-1}$ & $1.3 \times 10^{1}$ & $1.3 \times 10^{1}$ & $3.8 \times 10^{0}$ & $5.2 \times 10^{2}$ & $5.3 \times 10^{2}$ \\
\hline 1957 & $2.1 \times 10^{-1}$ & $2.9 \times 10^{2}$ & $1.4 \times 10^{-1}$ & $2.5 \times 10^{0}$ & $2.6 \times 10^{0}$ & $3.8 \times 10^{0}$ & $9.7 \times 10^{1}$ & $1.0 \times 10^{2}$ \\
\hline 1958 & $2.1 \times 10^{-1}$ & $2.0 \times 10^{1}$ & $1.4 \times 10^{-1}$ & $1.7 \times 10^{-1}$ & $3.1 \times 10^{-1}$ & $3.8 \times 10^{0}$ & $6.6 \times 10^{0}$ & $1.0 \times 10^{1}$ \\
\hline 1959 & $2.1 \times 10^{-1}$ & $1.6 \times 10^{2}$ & $1.4 \times 10^{-1}$ & $1.4 \times 10^{0}$ & $1.5 \times 10^{0}$ & $3.8 \times 10^{0}$ & $5.4 \times 10^{1}$ & $5.7 \times 10^{1}$ \\
\hline 1960 & $2.1 \times 10^{-1}$ & $7.3 \times 10^{0}$ & $1.4 \times 10^{-1}$ & $6.1 \times 10^{-2}$ & $2.0 \times 10^{-1}$ & $3.8 \times 10^{0}$ & $2.4 \times 10^{0}$ & $6.2 \times 10^{0}$ \\
\hline 1961 & $2.1 \times 10^{-1}$ & $1.6 \times 10^{2}$ & $1.4 \times 10^{-1}$ & $1.4 \times 10^{0}$ & $1.5 \times 10^{0}$ & $3.8 \times 10^{0}$ & $5.4 \times 10^{1}$ & $5.7 \times 10^{1}$ \\
\hline 1962 & $2.1 \times 10^{-1}$ & $1.7 \times 10^{1}$ & $1.4 \times 10^{-1}$ & $1.4 \times 10^{-1}$ & $2.8 \times 10^{-1}$ & $3.8 \times 10^{0}$ & $5.5 \times 10^{0}$ & $9.3 \times 10^{0}$ \\
\hline 1963 & $2.1 \times 10^{-1}$ & $4.8 \times 10^{0}$ & $1.4 \times 10^{-1}$ & $4.0 \times 10^{-2}$ & $1.8 \times 10^{-1}$ & $3.8 \times 10^{0}$ & $1.6 \times 10^{0}$ & $5.4 \times 10^{0}$ \\
\hline 1964 & $2.1 \times 10^{-1}$ & $1.2 \times 10^{1}$ & $1.4 \times 10^{-1}$ & $9.7 \times 10^{-2}$ & $2.4 \times 10^{-1}$ & $3.8 \times 10^{0}$ & $3.8 \times 10^{0}$ & $7.6 \times 10^{0}$ \\
\hline 1965 & $2.1 \times 10^{-1}$ & $1.8 \times 10^{1}$ & $1.4 \times 10^{-1}$ & $1.5 \times 10^{-1}$ & $2.9 \times 10^{-1}$ & $3.8 \times 10^{0}$ & $6.0 \times 10^{0}$ & $9.8 \times 10^{0}$ \\
\hline 1966 & $2.1 \times 10^{-1}$ & $3.2 \times 10^{1}$ & $1.4 \times 10^{-1}$ & $2.7 \times 10^{-1}$ & $4.1 \times 10^{-1}$ & $3.8 \times 10^{0}$ & $1.0 \times 10^{1}$ & $1.4 \times 10^{1}$ \\
\hline 1967 & $2.1 \times 10^{-1}$ & $2.0 \times 10^{1}$ & $1.4 \times 10^{-1}$ & $1.7 \times 10^{-1}$ & $3.1 \times 10^{-1}$ & $3.8 \times 10^{0}$ & $6.6 \times 10^{0}$ & $1.0 \times 10^{1}$ \\
\hline 1968 & $2.1 \times 10^{-1}$ & $2.2 \times 10^{1}$ & $1.4 \times 10^{-1}$ & $1.8 \times 10^{-1}$ & $3.3 \times 10^{-1}$ & $3.8 \times 10^{0}$ & $7.3 \times 10^{0}$ & $1.1 \times 10^{1}$ \\
\hline 1969 & $2.1 \times 10^{-1}$ & $3.6 \times 10^{1}$ & $1.4 \times 10^{-1}$ & $3.0 \times 10^{-1}$ & $4.4 \times 10^{-1}$ & $3.8 \times 10^{0}$ & $1.2 \times 10^{1}$ & $1.6 \times 10^{1}$ \\
\hline 1970 & $2.1 \times 10^{-1}$ & $3.4 \times 10^{1}$ & $1.4 \times 10^{-1}$ & $2.9 \times 10^{-1}$ & $4.3 \times 10^{-1}$ & $3.8 \times 10^{0}$ & $1.1 \times 10^{1}$ & $1.5 \times 10^{1}$ \\
\hline 1971 & $2.1 \times 10^{-1}$ & $2.7 \times 10^{1}$ & $1.4 \times 10^{-1}$ & $2.3 \times 10^{-1}$ & $3.7 \times 10^{-1}$ & $3.8 \times 10^{0}$ & $8.9 \times 10^{0}$ & $1.3 \times 10^{1}$ \\
\hline 1972 & $2.1 \times 10^{-1}$ & $2.7 \times 10^{0}$ & $1.4 \times 10^{-1}$ & $2.3 \times 10^{-2}$ & $1.6 \times 10^{-1}$ & $3.8 \times 10^{0}$ & $8.9 \times 10^{-1}$ & $4.7 \times 10^{0}$ \\
\hline 1973 & $2.1 \times 10^{-1}$ & $1.9 \times 10^{0}$ & $1.4 \times 10^{-1}$ & $1.6 \times 10^{-2}$ & $1.6 \times 10^{-1}$ & $3.8 \times 10^{0}$ & $6.3 \times 10^{-1}$ & $4.4 \times 10^{0}$ \\
\hline 1974 & $1.7 \times 10^{-1}$ & $1.9 \times 10^{0}$ & $1.2 \times 10^{-1}$ & $1.6 \times 10^{-2}$ & $1.3 \times 10^{-1}$ & $3.1 \times 10^{0}$ & $6.3 \times 10^{-1}$ & $3.7 \times 10^{0}$ \\
\hline 1975 & $1.4 \times 10^{-1}$ & $1.2 \times 10^{-1}$ & $9.5 \times 10^{-2}$ & $1.0 \times 10^{-3}$ & $9.6 \times 10^{-2}$ & $2.5 \times 10^{0}$ & $4.1 \times 10^{-2}$ & $2.6 \times 10^{0}$ \\
\hline 1976 & $1.5 \times 10^{-1}$ & $1.6 \times 10^{-1}$ & $1.0 \times 10^{-1}$ & $1.3 \times 10^{-3}$ & $1.0 \times 10^{-1}$ & $2.7 \times 10^{0}$ & $5.3 \times 10^{-2}$ & $2.8 \times 10^{0}$ \\
\hline 1977 & $1.4 \times 10^{-1}$ & $6.1 \times 10^{-2}$ & $9.5 \times 10^{-2}$ & $5.1 \times 10^{-4}$ & $9.5 \times 10^{-2}$ & $2.5 \times 10^{0}$ & $2.0 \times 10^{-2}$ & $2.6 \times 10^{0}$ \\
\hline 1978 & $1.3 \times 10^{-1}$ & $6.5 \times 10^{-2}$ & $8.8 \times 10^{-2}$ & $5.5 \times 10^{-4}$ & $8.9 \times 10^{-2}$ & $2.4 \times 10^{0}$ & $2.2 \times 10^{-2}$ & $2.4 \times 10^{0}$ \\
\hline 1979 & $1.3 \times 10^{-1}$ & $8.4 \times 10^{-2}$ & $8.8 \times 10^{-2}$ & $7.1 \times 10^{-4}$ & $8.9 \times 10^{-2}$ & $2.4 \times 10^{0}$ & $2.8 \times 10^{-2}$ & $2.4 \times 10^{0}$ \\
\hline 1980 & $1.6 \times 10^{-1}$ & $2.5 \times 10^{-2}$ & $1.1 \times 10^{-1}$ & $2.1 \times 10^{-4}$ & $1.1 \times 10^{-1}$ & $2.9 \times 10^{0}$ & $8.3 \times 10^{-3}$ & $2.9 \times 10^{0}$ \\
\hline 1981 & $1.6 \times 10^{-1}$ & $4.7 \times 10^{-2}$ & $1.1 \times 10^{-1}$ & $3.9 \times 10^{-4}$ & $1.1 \times 10^{-1}$ & $2.9 \times 10^{0}$ & $1.6 \times 10^{-2}$ & $2.9 \times 10^{0}$ \\
\hline 1982 & $5.9 \times 10^{-2}$ & $1.1 \times 10^{-1}$ & $4.0 \times 10^{-2}$ & $9.2 \times 10^{-4}$ & $4.1 \times 10^{-2}$ & $1.1 \times 10^{0}$ & $3.6 \times 10^{-2}$ & $1.1 \times 10^{0}$ \\
\hline 1983 & $4.1 \times 10^{-2}$ & $8.4 \times 10^{-2}$ & $2.8 \times 10^{-2}$ & $7.1 \times 10^{-4}$ & $2.8 \times 10^{-2}$ & $7.4 \times 10^{-1}$ & $2.8 \times 10^{-2}$ & $7.7 \times 10^{-1}$ \\
\hline 1984 & $3.5 \times 10^{-2}$ & $2.8 \times 10^{-1}$ & $2.4 \times 10^{-2}$ & $2.4 \times 10^{-3}$ & $2.6 \times 10^{-2}$ & $6.3 \times 10^{-1}$ & $9.3 \times 10^{-2}$ & $7.3 \times 10^{-1}$ \\
\hline 1985 & $6.5 \times 10^{-2}$ & $6.0 \times 10^{-2}$ & $4.4 \times 10^{-2}$ & $5.0 \times 10^{-4}$ & $4.5 \times 10^{-2}$ & $1.2 \times 10^{0}$ & $2.0 \times 10^{-2}$ & $1.2 \times 10^{0}$ \\
\hline 1986 & $8.7 \times 10^{-2}$ & $2.6 \times 10^{-2}$ & $5.9 \times 10^{-2}$ & $2.2 \times 10^{-4}$ & $5.9 \times 10^{-2}$ & $1.6 \times 10^{0}$ & $8.6 \times 10^{-3}$ & $1.6 \times 10^{0}$ \\
\hline 1987 & $7.2 \times 10^{-2}$ & $1.3 \times 10^{-2}$ & $4.9 \times 10^{-2}$ & $1.1 \times 10^{-4}$ & $4.9 \times 10^{-2}$ & $1.3 \times 10^{0}$ & $4.3 \times 10^{-3}$ & $1.3 \times 10^{0}$ \\
\hline 1988 & $6.3 \times 10^{-2}$ & $2.6 \times 10^{-3}$ & $4.3 \times 10^{-2}$ & $2.2 \times 10^{-5}$ & $4.3 \times 10^{-2}$ & $1.1 \times 10^{0}$ & $8.6 \times 10^{-4}$ & $1.1 \times 10^{0}$ \\
\hline 1989 & $5.2 \times 10^{-2}$ & $3.4 \times 10^{-4}$ & $3.5 \times 10^{-2}$ & $2.9 \times 10^{-6}$ & $3.5 \times 10^{-2}$ & $9.4 \times 10^{-1}$ & $1.1 \times 10^{-4}$ & $9.4 \times 10^{-1}$ \\
\hline 1990 & $1.3 \times 10^{-2}$ & $1.2 \times 10^{-4}$ & $8.8 \times 10^{-3}$ & $1.0 \times 10^{-6}$ & $8.8 \times 10^{-3}$ & $2.4 \times 10^{-1}$ & $4.1 \times 10^{-5}$ & $2.4 \times 10^{-1}$ \\
\hline 1991 & $1.0 \times 10^{-2}$ & $3.0 \times 10^{-5}$ & $6.8 \times 10^{-3}$ & $2.5 \times 10^{-7}$ & $6.8 \times 10^{-3}$ & $1.8 \times 10^{-1}$ & $9.9 \times 10^{-6}$ & $1.8 \times 10^{-1}$ \\
\hline 1992 & $3.5 \times 10^{-3}$ & $1.0 \times 10^{-4}$ & $2.4 \times 10^{-3}$ & $8.4 \times 10^{-7}$ & $2.4 \times 10^{-3}$ & $6.3 \times 10^{-2}$ & $3.3 \times 10^{-5}$ & $6.3 \times 10^{-2}$ \\
\hline 1993 & $5.0 \times 10^{-4}$ & $1.5 \times 10^{-4}$ & $3.4 \times 10^{-4}$ & $1.2 \times 10^{-6}$ & $3.4 \times 10^{-4}$ & $9.0 \times 10^{-3}$ & $4.9 \times 10^{-5}$ & $9.0 \times 10^{-3}$ \\
\hline 1994 & $3.8 \times 10^{-3}$ & $7.0 \times 10^{-5}$ & $2.6 \times 10^{-3}$ & $5.9 \times 10^{-7}$ & $2.6 \times 10^{-3}$ & $6.9 \times 10^{-2}$ & $2.3 \times 10^{-5}$ & $6.9 \times 10^{-2}$ \\
\hline 1995 & $4.7 \times 10^{-3}$ & $2.1 \times 10^{-2}$ & $3.2 \times 10^{-3}$ & $1.7 \times 10^{-4}$ & $3.4 \times 10^{-3}$ & $8.5 \times 10^{-2}$ & $6.8 \times 10^{-3}$ & $9.2 \times 10^{-2}$ \\
\hline 1996 & $1.0 \times 10^{-2}$ & $8.7 \times 10^{-5}$ & $7.0 \times 10^{-3}$ & $7.3 \times 10^{-7}$ & $7.0 \times 10^{-3}$ & $1.9 \times 10^{-1}$ & $2.9 \times 10^{-5}$ & $1.9 \times 10^{-1}$ \\
\hline Total & $5.7 \times 10^{0}$ & $2.5 \times 10^{3}$ & $3.9 \times 10^{0}$ & $2.1 \times 10^{1}$ & $2.5 \times 10^{1}$ & $1.0 \times 10^{2}$ & $8.3 \times 10^{2}$ & $9.4 \times 10^{2}$ \\
\hline
\end{tabular}


Table 4-10. Liquid I-131 Releases and Dose

\begin{tabular}{|c|c|c|c|c|c|c|c|c|c|c|c|}
\hline Year & $\begin{array}{c}\text { River } \\
\text { FLow } \\
\text { Rate } \\
\left(\mathrm{m}^{3} / \mathrm{s}\right)\end{array}$ & \begin{tabular}{|c|}
$\mathrm{BJ}$ \\
Derived \\
Flow \\
Rate \\
$\left(\mathrm{m}^{3} / \mathrm{s}\right)$
\end{tabular} & \begin{tabular}{|c|} 
PW \\
Derived \\
Flow \\
Rate \\
$\left(\mathrm{m}^{3} / \mathrm{s}\right)$ \\
\end{tabular} & $\begin{array}{l}\text { Releases } \\
\text { to } \\
\text { Streams } \\
\text { (Ci) }\end{array}$ & $\begin{array}{c}\text { Max } \\
\text { Ind } \\
\text { Below } \\
\text { SRS } \\
\text { (mrem) }\end{array}$ & $\begin{array}{c}\text { Max } \\
\text { Ind } \\
\text { BJ } \\
\text { (mrem) }\end{array}$ & $\begin{array}{c}\text { Max } \\
\text { Ind } \\
\text { PW } \\
\text { (mrem) }\end{array}$ & $\begin{array}{c}\text { Pop } \\
\text { Dose } \\
\text { BJ } \\
\text { (per-rem) }\end{array}$ & $\begin{array}{c}\text { Pop } \\
\text { Dose } \\
\text { PW } \\
\text { (per-rem) }\end{array}$ & $\begin{array}{c}\text { Pop } \\
\text { Dose } \\
80 \mathrm{~km} \\
\text { (per-rem) }\end{array}$ & $\begin{array}{c}\text { Pop } \\
\text { Dose } \\
\text { Total } \\
\text { (per-rem) }\end{array}$ \\
\hline 1954 & 209 & & 263 & & & & & & & & \\
\hline 1955 & 169 & & 213 & & & & & & & & \\
\hline 1956 & 179 & & 225 & & & & & & & & \\
\hline 1957 & 235 & & 297 & $6.5 \times 10^{1}$ & $4.1 \times 10^{-1}$ & & $2.4 \times 10^{-1}$ & & $1.4 \times 10^{0}$ & $1.0 \times 10^{0}$ & $2.4 \times 10^{0}$ \\
\hline 1958 & 313 & & 394 & & & & & & & & \\
\hline 1959 & 276 & & 348 & & & & & & & & \\
\hline 1960 & 371 & & 468 & $2.4 \times 10^{1}$ & $9.5 \times 10^{-2}$ & & $5.5 \times 10^{-2}$ & & $3.3 \times 10^{-1}$ & $2.4 \times 10^{-1}$ & $5.7 \times 10^{-1}$ \\
\hline 1961 & 309 & & 389 & $2.9 \times 10^{1}$ & $1.4 \times 10^{-1}$ & & $8.0 \times 10^{-2}$ & & $4.9 \times 10^{-1}$ & $3.5 \times 10^{-1}$ & $8.3 \times 10^{-1}$ \\
\hline 1962 & 300 & & 377 & $8.7 \times 10^{1}$ & $4.3 \times 10^{-1}$ & & $2.5 \times 10^{-1}$ & & $1.5 \times 10^{0}$ & $1.1 \times 10^{0}$ & $2.6 \times 10^{0}$ \\
\hline 1963 & 315 & & 397 & $4.6 \times 10^{1}$ & $2.1 \times 10^{-1}$ & & $1.2 \times 10^{-1}$ & & $7.5 \times 10^{-1}$ & $5.4 \times 10^{-1}$ & $1.3 \times 10^{0}$ \\
\hline 1964 & 580 & & 731 & $8.7 \times 10^{0}$ & $2.2 \times 10^{-2}$ & & $1.3 \times 10^{-2}$ & & $7.7 \times 10^{-2}$ & $5.5 \times 10^{-2}$ & $1.3 \times 10^{-1}$ \\
\hline 1965 & 362 & 800 & 456 & $5.4 \times 10^{0}$ & $2.2 \times 10^{-2}$ & $7.3 \times 10^{-3}$ & $1.3 \times 10^{-2}$ & $1.5 \times 10^{-1}$ & $7.7 \times 10^{-2}$ & $5.5 \times 10^{-2}$ & $2.8 \times 10^{-1}$ \\
\hline 1966 & $\overline{316}$ & 520 & 399 & $3.0 \times 10^{0}$ & $1.4 \times 10^{-2}$ & $6.2 \times 10^{-3}$ & $8.1 \times 10^{-3}$ & $1.3 \times 10^{-1}$ & $4.9 \times 10^{-2}$ & $3.5 \times 10^{-2}$ & $2.1 \times 10^{-1}$ \\
\hline 1967 & 299 & 625 & 377 & $1.4 \times 10^{1}$ & $6.9 \times 10^{-2}$ & $2.4 \times 10^{-2}$ & $4.0 \times 10^{-2}$ & $4.9 \times 10^{-1}$ & $2.4 \times 10^{-14}$ & $1.7 \times 10^{-1}$ & $9.1 \times 10^{-1}$ \\
\hline 1968 & 273 & 470 & 343 & $9.5 \times 10^{0}$ & $5.1 \times 10^{-2}$ & $2.2 \times 10^{-2}$ & $3.0 \times 10^{-2}$ & $4.5 \times 10^{-1}$ & $1.8 \times 10^{-1}$ & $1.3 \times 10^{-1}$ & $7.5 \times 10^{-1}$ \\
\hline 1969 & 310 & 624 & 390 & $5.0 \times 10^{0}$ & $2.4 \times 10^{-2}$ & $8.6 \times 10^{-3}$ & $1.4 \times 10^{-2}$ & $1.8 \times 10^{-1}$ & $8.3 \times 10^{-2}$ & $5.9 \times 10^{-2}$ & $3.2 \times 10^{-1}$ \\
\hline 1970 & 232 & 772 & 293 & $2.2 \times 10^{0}$ & $1.4 \times 10^{-2}$ & $3.1 \times 10^{-3}$ & $8.1 \times 10^{-3}$ & $6.3 \times 10^{-2}$ & $4.9 \times 10^{-2}$ & $3.5 \times 10^{-2}$ & $1.5 \times 10^{-1}$ \\
\hline 1971 & 303 & 1245 & 381 & $1.5 \times 10^{0}$ & $7.3 \times 10^{-3}$ & $1.3 \times 10^{-3}$ & $4.2 \times 10^{-3}$ & $2.7 \times 10^{-2}$ & $2.6 \times 10^{-2}$ & $1.8 \times 10^{-2}$ & $7.0 \times 10^{-2}$ \\
\hline 1972 & 318 & 775 & 401 & $9.3 \times 10^{-1}$ & $4.3 \times 10^{-3}$ & $1.3 \times 10^{-3}$ & $2.5 \times 10^{-3}$ & $2.6 \times 10^{-2}$ & $1.5 \times 10^{-2}$ & $1.1 \times 10^{-2}$ & $5.2 \times 10^{-2}$ \\
\hline 1973 & 409 & 656 & 468 & $1.6 \times 10^{-1}$ & $5.8 \times 10^{-4}$ & $2.6 \times 10^{-4}$ & $3.7 \times 10^{-4}$ & $5.4 \times 10^{-3}$ & $2.2 \times 10^{-3}$ & $1.4 \times 10^{-3}$ & $9.0 \times 10^{-3}$ \\
\hline 1974 & 314 & 640 & 373 & $1.4 \times 10^{-2}$ & $6.6 \times 10^{-5}$ & $2.4 \times 10^{-5}$ & $4.0 \times 10^{-5}$ & $4.8 \times 10^{-4}$ & $2.4 \times 10^{-4}$ & $1.6 \times 10^{-4}$ & $8.9 \times 10^{-4}$ \\
\hline 1975 & 436 & 877 & 538 & $1.2 \times 10^{-1}$ & $4.1 \times 10^{-4}$ & $1.5 \times 10^{-4}$ & $2.4 \times 10^{-4}$ & $3.0 \times 10^{-3}$ & $1.5 \times 10^{-3}$ & $1.0 \times 10^{-3}$ & $5.5 \times 10^{-3}$ \\
\hline 1976 & 394 & 996 & 472 & $2.0 \times 10^{-3}$ & $7.5 \times 10^{-6}$ & $2.2 \times 10^{-6}$ & $4.6 \times 10^{-6}$ & $4.4 \times 10^{-5}$ & $2.8 \times 10^{-5}$ & $1.9 \times 10^{-5}$ & $9.1 \times 10^{-5}$ \\
\hline 1977 & 330 & 562 & 433 & $1.0 \times 10^{-2}$ & $4.5 \times 10^{-5}$ & $1.9 \times 10^{-5}$ & $2.5 \times 10^{-5}$ & $3.9 \times 10^{-4}$ & $1.5 \times 10^{-4}$ & $1.1 \times 10^{-4}$ & $6.5 \times 10^{-4}$ \\
\hline 1978 & 298 & 689 & 324 & $7.3 \times 10^{-4}$ & $3.6 \times 10^{-6}$ & $1.1 \times 10^{-6}$ & $2.4 \times 10^{-6}$ & $2.3 \times 10^{-5}$ & $1.5 \times 10^{-5}$ & $9.0 \times 10^{-6}$ & $4.7 \times 10^{-5}$ \\
\hline 1979 & 375 & 989 & 495 & & & & & & & & \\
\hline 1980 & 374 & 863 & 488 & & & & & & & & \\
\hline 1981 & 187 & 331 & 243 & & & & & & & & \\
\hline 1982 & 203 & 555 & 287 & & & & & & & & \\
\hline 1983 & 350 & 361 & 417 & & & & & & & & \\
\hline 1984 & 361 & 747 & 487 & & & & & & & & \\
\hline 1985 & 203 & 263 & 254 & & & & & & & & \\
\hline 1986 & 175 & 235 & 213 & & & & & & & & \\
\hline 1987 & 254 & 374 & 357 & & & & & & & & \\
\hline 1988 & 152 & 211 & 203 & & & & & & & & \\
\hline 1989 & 226 & 240 & 245 & & & & & & & & \\
\hline 1990 & 336 & 447 & 367 & & & & & & & & \\
\hline 1991 & 328 & 550 & 453 & & & & & & & & \\
\hline 1992 & 331 & 350 & 279 & & & & & & & & \\
\hline 1993 & 419 & 566 & 416 & & & & & & & & \\
\hline 1994 & 347 & 421 & 348 & & & & & & & & \\
\hline 1995 & 361 & 438 & 369 & & & & & & & & \\
\hline 1996 & 325 & 310 & 287 & & & & & & & & \\
\hline Total & & & & $3.0 \times 10^{2}$ & $1.5 \times 10^{0}$ & $7.4 \times 10^{-2}$ & $8.8 \times 10^{-1}$ & $1.5 \times 10^{0}$ & $5.3 \times 10^{0}$ & $3.8 \times 10^{0}$ & $1.1 \times 10^{1}$ \\
\hline
\end{tabular}


Assessment of Radionuclides

in the Savannah River Site Environment-Summary (U)

WSRC-TR-98-00162

Table 4-11. Atmospheric Am-241 Releases and Dose

\begin{tabular}{|c|c|c|c|}
\hline Year & $\begin{array}{c}\text { Release } \\
(\mathrm{Ci})\end{array}$ & $\begin{array}{c}\text { Maximum Individual } \\
\text { Dose at } \\
\text { Boundary } \\
\text { (mrem) } \\
\end{array}$ & $\begin{array}{c}\text { Population } \\
\text { Dose } \\
\text { (per-rem) }\end{array}$ \\
\hline 1954 & & & \\
\hline 1955 & & & \\
\hline 1956 & & & \\
\hline 1957 & & & \\
\hline 1958 & & & \\
\hline 1959 & & & \\
\hline 1960 & & & \\
\hline 1961 & & & \\
\hline 1962 & & & \\
\hline 1963 & & & \\
\hline 1964 & & & \\
\hline 1965 & & & \\
\hline 1966 & & & \\
\hline 1967 & & & \\
\hline 1968 & & & \\
\hline 1969 & & & \\
\hline 1970 & & & \\
\hline 1971 & & & \\
\hline 1972 & & & \\
\hline 1973 & & & \\
\hline 1974 & & & \\
\hline 1975 & & & \\
\hline 1976 & & & \\
\hline 1977 & $3.4 \times 10^{-4}$ & $1.1 \times 10^{-3}$ & $6.5 \times 10^{-2}$ \\
\hline 1978 & $1.2 \times 10^{-3}$ & $4.1 \times 10^{-3}$ & $2.4 \times 10^{-1}$ \\
\hline 1979 & $3.6 \times 10^{-4}$ & $1.2 \times 10^{-3}$ & $6.8 \times 10^{-2}$ \\
\hline 1980 & $1.1 \times 10^{-3}$ & $3.6 \times 10^{-3}$ & $2.1 \times 10^{-1}$ \\
\hline 1981 & $4.9 \times 10^{-4}$ & $1.6 \times 10^{-3}$ & $9.5 \times 10^{-2}$ \\
\hline 1982 & $5.0 \times 10^{-4}$ & $1.7 \times 10^{-3}$ & $9.6 \times 10^{-2}$ \\
\hline 1983 & $2.6 \times 10^{-4}$ & $8.5 \times 10^{-4}$ & $4.9 \times 10^{-2}$ \\
\hline 1984 & $1.4 \times 10^{-4}$ & $4.7 \times 10^{-4}$ & $2.7 \times 10^{-2}$ \\
\hline 1985 & $4.3 \times 10^{-4}$ & $1.4 \times 10^{-3}$ & $8.2 \times 10^{-2}$ \\
\hline 1986 & $1.5 \times 10^{-4}$ & $5.1 \times 10^{-4}$ & $3.0 \times 10^{-2}$ \\
\hline 1987 & $2.0 \times 10^{-4}$ & $6.8 \times 10^{-4}$ & $3.9 \times 10^{-2}$ \\
\hline 1988 & $1.2 \times 10^{-4}$ & $3.9 \times 10^{-4}$ & $2.3 \times 10^{-2}$ \\
\hline 1989 & $2.0 \times 10^{-4}$ & $6.7 \times 10^{-4}$ & $3.9 \times 10^{-2}$ \\
\hline 1990 & $1.3 \times 10^{-4}$ & $4.3 \times 10^{-4}$ & $2.5 \times 10^{-2}$ \\
\hline 1991 & $1.5 \times 10^{-4}$ & $5.0 \times 10^{-4}$ & $2.9 \times 10^{-2}$ \\
\hline 1992 & $1.1 \times 10^{-4}$ & $3.8 \times 10^{-4}$ & $2.2 \times 10^{-2}$ \\
\hline 1993 & $1.4 \times 10^{-4}$ & $4.7 \times 10^{-4}$ & $2.7 \times 10^{-2}$ \\
\hline 1994 & $5.6 \times 10^{-5}$ & $1.9 \times 10^{-4}$ & $1.1 \times 10^{-2}$ \\
\hline 1995 & $3.0 \times 10^{-5}$ & $1.0 \times 10^{-4}$ & $5.8 \times 10^{-3}$ \\
\hline 1996 & $1.3 \times 10^{-5}$ & $4.3 \times 10^{-5}$ & $2.5 \times 10^{-3}$ \\
\hline Total & $6.1 \times 10^{-3}$ & $2.0 \times 10^{-2}$ & $1.2 \times 10^{0}$ \\
\hline
\end{tabular}


Chapter 4. Releases and Doses

of Individual Radionuclides

WSRC-TR-98-00162

Table 4-12. Atmospheric $\mathrm{Cm}-244$ Releases and Dose

\begin{tabular}{|c|c|c|c|}
\hline Year & Release & $\begin{array}{c}\text { Maximum Individual } \\
\text { Dose at Boundary } \\
\text { (mrem) }\end{array}$ & $\begin{array}{l}\text { Population } \\
\text { Dose } \\
\text { (per-rem) }\end{array}$ \\
\hline \multicolumn{4}{|l|}{1954} \\
\hline \multicolumn{4}{|l|}{1955} \\
\hline \multicolumn{4}{|l|}{1956} \\
\hline \multicolumn{4}{|l|}{1957} \\
\hline \multicolumn{4}{|l|}{1958} \\
\hline \multicolumn{4}{|l|}{1959} \\
\hline \multicolumn{4}{|l|}{1960} \\
\hline \multicolumn{4}{|l|}{1961} \\
\hline \multicolumn{4}{|l|}{1962} \\
\hline \multicolumn{4}{|l|}{1963} \\
\hline 1964 & $3.2 \times 10^{-2}$ & $5.4 \times 10^{-2}$ & $3.1 \times 10^{0}$ \\
\hline \multicolumn{4}{|l|}{1965} \\
\hline \multicolumn{4}{|l|}{1966} \\
\hline 1967 & $3.0 \times 10^{-2}$ & $5.1 \times 10^{-2}$ & $3.0 \times 10^{0}$ \\
\hline \multicolumn{4}{|l|}{1968} \\
\hline 1969 & $2.1 \times 10^{-2}$ & $3.6 \times 10^{-2}$ & $2.1 \times 10^{0}$ \\
\hline 1970 & $9.1 \times 10^{-4}$ & $1.6 \times 10^{-3}$ & $9.0 \times 10^{-2}$ \\
\hline 1971 & $7.0 \times 10^{-4}$ & $1.2 \times 10^{-3}$ & $6.9 \times 10^{-2}$ \\
\hline 1972 & $3.9 \times 10^{-4}$ & $6.7 \times 10^{-4}$ & $3.9 \times 10^{-2}$ \\
\hline 1973 & $3.8 \times 10^{-4}$ & $6.5 \times 10^{-4}$ & $3.8 \times 10^{-2}$ \\
\hline 1974 & $3.9 \times 10^{-4}$ & $6.7 \times 10^{-4}$ & $3.9 \times 10^{-2}$ \\
\hline \multicolumn{4}{|l|}{1975} \\
\hline 1976 & $3.3 \times 10^{-4}$ & $5.6 \times 10^{-4}$ & $3.3 \times 10^{-2}$ \\
\hline 1977 & $3.4 \times 10^{-4}$ & $5.8 \times 10^{-4}$ & $3.4 \times 10^{-2}$ \\
\hline 1978 & $1.4 \times 10^{-4}$ & $2.4 \times 10^{-4}$ & $1.4 \times 10^{-2}$ \\
\hline 1979 & $3.9 \times 10^{-4}$ & $6.7 \times 10^{-4}$ & $3.9 \times 10^{-2}$ \\
\hline 1980 & $9.0 \times 10^{-4}$ & $1.5 \times 10^{-3}$ & $8.9 \times 10^{-2}$ \\
\hline 1981 & $1.6 \times 10^{-4}$ & $2.8 \times 10^{-4}$ & $1.6 \times 10^{-2}$ \\
\hline 1982 & $1.7 \times 10^{-4}$ & $2.8 \times 10^{-4}$ & $1.6 \times 10^{-2}$ \\
\hline 1983 & $5.1 \times 10^{-4}$ & $8.7 \times 10^{-4}$ & $5.0 \times 10^{-2}$ \\
\hline 1984 & $2.6 \times 10^{-4}$ & $4.4 \times 10^{-4}$ & $2.6 \times 10^{-2}$ \\
\hline 1985 & $2.5 \times 10^{-4}$ & $4.2 \times 10^{-4}$ & $2.4 \times 10^{-2}$ \\
\hline 1986 & $2.8 \times 10^{-5}$ & $4.8 \times 10^{-5}$ & $2.8 \times 10^{-3}$ \\
\hline 1987 & $3.2 \times 10^{-4}$ & $5.5 \times 10^{-4}$ & $3.2 \times 10^{-2}$ \\
\hline 1988 & $6.7 \times 10^{-5}$ & $1.1 \times 10^{-4}$ & $6.6 \times 10^{-3}$ \\
\hline 1989 & $2.8 \times 10^{-5}$ & $4.8 \times 10^{-5}$ & $2.8 \times 10^{-3}$ \\
\hline 1990 & $2.0 \times 10^{-5}$ & $3.5 \times 10^{-5}$ & $2.0 \times 10^{-3}$ \\
\hline 1991 & $4.2 \times 10^{-5}$ & $7.3 \times 10^{-5}$ & $4.2 \times 10^{-3}$ \\
\hline 1992 & $2.3 \times 10^{-5}$ & $3.9 \times 10^{-5}$ & $2.3 \times 10^{-3}$ \\
\hline 1993 & $5.6 \times 10^{-5}$ & $9.7 \times 10^{-5}$ & $5.6 \times 10^{-3}$ \\
\hline 1994 & $1.6 \times 10^{-5}$ & $2.8 \times 10^{-5}$ & $1.6 \times 10^{-3}$ \\
\hline 1995 & $3.4 \times 10^{-6}$ & $5.8 \times 10^{-6}$ & $3.4 \times 10^{-4}$ \\
\hline 1996 & $1.3 \times 10^{-4}$ & $2.3 \times 10^{-4}$ & $1.3 \times 10^{-2}$ \\
\hline Total & $9.0 \times 10^{-2}$ & $1.5 \times 10^{-1}$ & $8.9 \times 10^{0}$ \\
\hline
\end{tabular}


Assessment of Radionuclides

Table 4-13. Liquid Cm-244 Releases and Dose

\begin{tabular}{|c|c|c|c|c|c|c|c|c|c|c|c|}
\hline Year & $\begin{array}{l}\text { River } \\
\text { Flow } \\
\text { Rate } \\
\left(\mathrm{m}^{3} / \mathrm{s}\right)\end{array}$ & \begin{tabular}{|c|} 
B-J \\
Derived \\
Flow \\
Rate \\
$\left(\mathrm{m}^{3} / \mathrm{s}\right)$
\end{tabular} & \begin{tabular}{|c|} 
PW \\
Derived \\
Flow \\
Rate \\
$\left(\mathrm{m}^{3} / \mathrm{s}\right)$
\end{tabular} & $\begin{array}{l}\text { Releases } \\
\text { to } \\
\text { Streams } \\
\text { (Ci) }\end{array}$ & $\begin{array}{c}\text { Max } \\
\text { Ind } \\
\text { Below SRS } \\
\text { (mrem) }\end{array}$ & $\begin{array}{l}\text { Max } \\
\text { Ind } \\
\text { BJ } \\
\text { (mrem) }\end{array}$ & $\begin{array}{c}\text { Max } \\
\text { Ind } \\
\text { PW } \\
\text { (mrem) }\end{array}$ & $\begin{array}{c}\text { Pop } \\
\text { Dose } \\
\text { BJ } \\
\text { (per-rem) }\end{array}$ & $\begin{array}{c}\text { Pop } \\
\text { Dose } \\
\text { PW } \\
\text { (per-rem) }\end{array}$ & $\begin{array}{c}\text { Pop } \\
\text { Dose } \\
80 \mathrm{~km} \\
\text { (per-rem) }\end{array}$ & $\begin{array}{c}\text { Pop } \\
\text { Dose } \\
\text { Total } \\
\text { (per-rem) }\end{array}$ \\
\hline 1954 & 209 & & 263 & & & & & & & & \\
\hline 1955 & 169 & & 213 & & & & & & & & \\
\hline 1956 & 179 & & 225 & & & & & & & & \\
\hline 1957 & 235 & & 297 & & & & & & & & \\
\hline 1958 & 313 & & 394 & & & & & & & & \\
\hline 1959 & 276 & & 348 & & & & & & & & \\
\hline 1960 & 371 & & 468 & & & & & & & & \\
\hline 1961 & 309 & & 389 & & & & & & & & \\
\hline 1962 & 300 & & 377 & & & & & & & & \\
\hline 1963 & 315 & & 397 & & & & & & & & \\
\hline 1964 & 580 & & 731 & & & & & & & & \\
\hline 1965 & 362 & 800 & 456 & & & & & & & & \\
\hline 1966 & 316 & 520 & 399 & & & & & & & & \\
\hline 1967 & 299 & 625 & 377 & & & & & & & & \\
\hline 1968 & 273 & 470 & 343 & & & & & & & & \\
\hline 1969 & 310 & 624 & 390 & $3.0 \times 10^{-2}$ & $8.5 \times 10^{-3}$ & $2.6 \times 10^{-3}$ & $4.1 \times 10^{-3}$ & $6.5 \times 10^{-2}$ & $3.0 \times 10^{-2}$ & $8.5 \times 10^{-1}$ & $9.4 \times 10^{-1}$ \\
\hline 1970 & 232 & 772 & 293 & & & & & & & & \\
\hline 1971 & 303 & 1245 & 381 & $3.2 \times 10^{-1}$ & $9.4 \times 10^{-2}$ & $1.4 \times 10^{-2}$ & $4.6 \times 10^{-2}$ & $3.5 \times 10^{-1}$ & $3.4 \times 10^{-1}$ & $9.4 \times 10^{0}$ & $1.0 \times 10^{1}$ \\
\hline 1972 & 318 & 775 & 401 & $2.8 \times 10^{-2}$ & $7.7 \times 10^{-3}$ & $1.9 \times 10^{-3}$ & $3.7 \times 10^{-3}$ & $4.9 \times 10^{-2}$ & $2.8 \times 10^{-2}$ & $7.7 \times 10^{-1}$ & $8.5 \times 10^{-1}$ \\
\hline 1973 & 409 & 656 & 468 & $3.6 \times 10^{-3}$ & $7.8 \times 10^{-4}$ & $3.0 \times 10^{-4}$ & $4.2 \times 10^{-4}$ & $7.5 \times 10^{-3}$ & $3.1 \times 10^{-3}$ & $7.8 \times 10^{-2}$ & $8.9 \times 10^{-2}$ \\
\hline 1974 & 314 & 640 & 373 & & & & & & & & \\
\hline 1975 & 436 & 877 & 538 & & & & & & & & \\
\hline 1976 & 394 & 996 & 472 & & & & & & & & \\
\hline 1977 & 330 & 562 & 433 & & & & & & & & \\
\hline 1978 & 298 & 689 & 324 & & & & & & & & 6 \\
\hline 1979 & 375 & 989 & 495 & & & & & & & & $\cdot$ \\
\hline 1980 & 374 & 863 & 488 & & & & & & & & \\
\hline 1981 & 187 & 331 & 243 & & & & & & & & \\
\hline 1982 & 203 & 555 & 287 & & & & & & & & \\
\hline 1983 & 350 & 361 & 417 & & & & & & & & \\
\hline 1984 & 361 & 747 & 487 & & & & & & & & \\
\hline 1985 & 203 & 263 & 254 & & & & & & & & \\
\hline 1986 & 175 & 235 & 213 & & & & & & & & \\
\hline 1987 & 254 & 374 & 357 & & & & & & & & \\
\hline 1988 & 152 & 211 & 203 & & & & & & & & \\
\hline 1989 & 226 & 240 & 245 & & & & & & & & \\
\hline 1990 & 336 & 447 & 367 & & & & & & & & \\
\hline 1991 & 328 & 550 & 453 & & & & & & & & \\
\hline 1992 & 331 & 350 & 279 & & & & & & & & \\
\hline 1993 & 419 & 566 & 416 & & & & & & & & \\
\hline 1994 & 347 & 421 & 348 & & & & & & & & \\
\hline 1995 & 361 & 438 & 369 & $3.6 \times 10^{-6}$ & $8.7 \times 10^{-7}$ & $4.4 \times 10^{-7}$ & $5.2 \times 10^{-7}$ & $1.1 \times 10^{-5}$ & $3.9 \times 10^{-6}$ & $8.7 \times 10^{-5}$ & $1.0 \times 10^{-4}$ \\
\hline 1996 & 325 & 310 & 287 & $1.2 \times 10^{-5}$ & $3.2 \times 10^{-6}$ & $2.1 \times 10^{-6}$ & $2.2 \times 10^{-6}$ & $5.3 \times 10^{-5}$ & $1.7 \times 10^{-5}$ & $3.2 \times 10^{-4}$ & $3.9 \times 10^{-4}$ \\
\hline Total & & & & $3.9 \times 10^{-1}$ & $1.1 \times 10^{-1}$ & $1.9 \times 10^{-2}$ & $5.4 \times 10^{-2}$ & $4.8 \times 10^{-1}$ & $4.0 \times 10^{-1}$ & $1.1 \times 10^{1}$ & $1.2 \times 10^{1}$ \\
\hline
\end{tabular}


Table 4-14. Atmospheric Ar-41 Releases and Dose

\begin{tabular}{|c|c|c|c|}
\hline Year & $\begin{array}{l}\text { Release } \\
\text { (Ci) }\end{array}$ & $\begin{array}{l}\text { Maximum Individual } \\
\text { Dose at Boundary } \\
\text { (mrem) }\end{array}$ & $\begin{array}{l}\text { Population Dose } \\
\text { (per-rem) }\end{array}$ \\
\hline 1954 & $5.0 \times 10^{4}$ & $6.2 \times 10^{-2}$ & $1.5 \times 10^{0}$ \\
\hline 1955 & $1.4 \times 10^{5}$ & $1.7 \times 10^{-1}$ & $4.2 \times 10^{0}$ \\
\hline 1956 & $2.5 \times 10^{5}$ & $3.1 \times 10^{-1}$ & $7.5 \times 10^{0}$ \\
\hline 1957 & $2.9 \times 10^{5}$ & $3.6 \times 10^{-1}$ & $8.7 \times 10^{0}$ \\
\hline 1958 & $3.4 \times 10^{5}$ & $4.2 \times 10^{-1}$ & $1.0 \times 10^{1}$ \\
\hline 1959 & $4.4 \times 10^{5}$ & $5.4 \times 10^{-1}$ & $1.3 \times 10^{f}$ \\
\hline 1960 & $4.0 \times 10^{5}$ & $4.9 \times 10^{-1}$ & $1.2 \times 10^{1}$ \\
\hline 1961 & $4.2 \times 10^{5}$ & $5.2 \times 10^{-1}$ & $1.3 \times 10^{1}$ \\
\hline 1962 & $4.3 \times 10^{5}$ & $5.3 \times 10^{-1}$ & $1.3 \times 10^{9}$ \\
\hline 1963 & $4.5 \times 10^{5}$ & $5.5 \times 10^{-1}$ & $1.4 \times 10^{1}$ \\
\hline 1964 & $3.7 \times 10^{5}$ & $4.6 \times 10^{-1}$ & $1.1 \times 10^{1}$ \\
\hline 1965 & $2.7 \times 10^{5}$ & $3.3 \times 10^{-1}$ & $8.1 \times 10^{0}$ \\
\hline 1966 & $2.8 \times 10^{5}$ & $3.4 \times 10^{-1}$ & $8.4 \times 10^{0}$ \\
\hline 1967 & $3.2 \times 10^{5}$ & $3.9 \times 10^{-1}$ & $9.6 \times 10^{0}$ \\
\hline 1968 & $2.2 \times 10^{5}$ & $2.7 \times 10^{-1}$ & $6.6 \times 10^{\circ}$ \\
\hline 1969 & $1.4 \times 10^{5}$ & $1.7 \times 10^{-1}$ & $4.2 \times 10^{0}$ \\
\hline 1970 & $1.1 \times 10^{5}$ & $1.4 \times 10^{-1}$ & $3.3 \times 10^{0}$ \\
\hline 1971 & $1.4 \times 10^{5}$ & $1.7 \times 10^{-1}$ & $4.2 \times 10^{\circ}$ \\
\hline 1972 & $1.7 \times 10^{5}$ & $2.1 \times 10^{-1}$ & $5.0 \times 10^{0}$ \\
\hline 1973 & $1.9 \times 10^{5}$ & $2.3 \times 10^{-1}$ & $5.6 \times 10^{0}$ \\
\hline 1974 & $1.1 \times 10^{5}$ & $1.3 \times 10^{-1}$ & $3.2 \times 10^{0}$ \\
\hline 1975 & $6.5 \times 10^{4}$ & $8.0 \times 10^{-2}$ & $2.0 \times 10^{0}$ \\
\hline 1976 & $8.3 \times 10^{4}$ & $1.0 \times 10^{-1}$ & $2.5 \times 10^{0}$ \\
\hline 1977 & $6.5 \times 10^{4}$ & $8.0 \times 10^{-2}$ & $2.0 \times 10^{0}$ \\
\hline 1978 & $5.2 \times 10^{4}$ & $6.4 \times 10^{-2}$ & $1.6 \times 10^{0}$ \\
\hline 1979 & $5.4 \times 10^{4}$ & $6.6 \times 10^{-2}$ & $1.6 \times 10^{0}$ \\
\hline 1980 & $7.0 \times 10^{4}$ & $8.6 \times 10^{-2}$ & $2.1 \times 10^{0}$ \\
\hline 1981 & $6.3 \times 10^{4}$ & $7.7 \times 10^{-2}$ & $1.9 \times 10^{0}$ \\
\hline 1982 & $6.0 \times 10^{4}$ & $7.4 \times 10^{-2}$ & $1.8 \times 10^{0}$ \\
\hline 1983 & $4.2 \times 10^{4}$ & $5.1 \times 10^{-2}$ & $1.2 \times 10^{0}$ \\
\hline 1984 & $3.6 \times 10^{4}$ & $4.4 \times 10^{-2}$ & $1.1 \times 10^{0}$ \\
\hline 1985 & $5.1 \times 10^{4}$ & $6.3 \times 10^{-2}$ & $1.5 \times 10^{0}$ \\
\hline 1986 & $8.3 \times 10^{4}$ & $1.0 \times 10^{-1}$ & $2.5 \times 10^{0}$ \\
\hline 1987 & $8.8 \times 10^{4}$ & $1.1 \times 10^{-1}$ & $2.6 \times 10^{0}$ \\
\hline 1988 & $3.0 \times 10^{4}$ & $3.6 \times 10^{-2}$ & $8.9 \times 10^{-1}$ \\
\hline \multicolumn{4}{|l|}{1989} \\
\hline \multicolumn{4}{|l|}{1990} \\
\hline \multicolumn{4}{|l|}{1991} \\
\hline 1992 & $2.5 \times 10^{2}$ & $3.1 \times 10^{-4}$ & $7.5 \times 10^{-3}$ \\
\hline \multicolumn{4}{|l|}{1993} \\
\hline \multicolumn{4}{|l|}{1994} \\
\hline \multicolumn{4}{|l|}{1995} \\
\hline \multicolumn{4}{|l|}{1996} \\
\hline Total & $6.4 \times 10^{6}$ & $7.8 \times 10^{0}$ & $1.9 \times 10^{2}$ \\
\hline
\end{tabular}


Table 4-15. Atmospheric Plutonium Releases and Dose

\begin{tabular}{|c|c|c|c|c|c|c|}
\hline Year & $\begin{array}{c}\text { Pu-238 } \\
\text { Release } \\
\text { (Ci) }\end{array}$ & $\begin{array}{c}\text { Pu-239 } \\
\text { Release } \\
\text { (Ci) }\end{array}$ & $\begin{array}{l}\text { Unidentified } \\
\text { Alpha } \\
\text { Release } \\
\text { (Ci) }\end{array}$ & $\begin{array}{c}\text { Total } \\
\text { Pu } \\
\text { Release } \\
\text { (Ci) }\end{array}$ & $\begin{array}{l}\text { Maximum } \\
\text { Individual } \\
\text { Dose at } \\
\text { Boundary } \\
\text { (mrem) }\end{array}$ & $\begin{array}{c}\text { Population } \\
\text { Dose } \\
\text { (per-rem) }\end{array}$ \\
\hline \multicolumn{7}{|l|}{1954} \\
\hline 1955 & & $2.7 \times 10^{0}$ & & $2.7 \times 10^{0}$ & $8.5 \times 10^{0}$ & $5.0 \times 10^{2}$ \\
\hline 1956 & & $3.1 \times 10^{-2}$ & & $3.1 \times 10^{-2}$ & $1.0 \times 10^{-1}$ & $5.8 \times 10^{0}$ \\
\hline 1957 & & $4.3 \times 10^{-2}$ & & $4.3 \times 10^{-2}$ & $1.4 \times 10^{-1}$ & $8.0 \times 10^{0}$ \\
\hline 1958 & & $2.1 \times 10^{-2}$ & & $2.1 \times 10^{-2}$ & $6.7 \times 10^{-2}$ & $3.9 \times 10^{0}$ \\
\hline 1959 & & $2.4 \times 10^{-2}$ & & $2.4 \times 10^{-2}$ & $7.7 \times 10^{-2}$ & $4.5 \times 10^{0}$ \\
\hline 1960 & & $7.1 \times 10^{-2}$ & & $7.1 \times 10^{-2}$ & $2.3 \times 10^{-1}$ & $1.3 \times 10^{1}$ \\
\hline 1961 & & $1.2 \times 10^{-2}$ & $2.2 \times 10^{-4}$ & $1.2 \times 10^{-2}$ & $3.9 \times 10^{-2}$ & $2.3 \times 10^{0}$ \\
\hline 1962 & & $1.1 \times 10^{-2}$ & $3.0 \times 10^{-4}$ & $1.1 \times 10^{-2}$ & $3.6 \times 10^{-2}$ & $2.1 \times 10^{0}$ \\
\hline 1963 & & $3.5 \times 10^{-3}$ & $2.4 \times 10^{-4}$ & $3.7 \times 10^{-3}$ & $1.2 \times 10^{-2}$ & $7.0 \times 10^{-1}$ \\
\hline 1964 & & $5.6 \times 10^{-3}$ & & $5.6 \times 10^{-3}$ & $1.8 \times 10^{-2}$ & $1.0 \times 10^{0}$ \\
\hline 1965 & & $1.7 \times 10^{-2}$ & $5.2 \times 10^{-2}$ & $6.8 \times 10^{-2}$ & $2.2 \times 10^{-1}$ & $1.3 \times 10^{1}$ \\
\hline 1966 & & $1.0 \times 10^{-2}$ & $2.1 \times 10^{-2}$ & $3.1 \times 10^{-2}$ & $1.0 \times 10^{-1}$ & $5.8 \times 10^{0}$ \\
\hline 1967 & $2.6 \times 10^{-4}$ & $1.0 \times 10^{-2}$ & $3.0 \times 10^{-4}$ & $1.1 \times 10^{-2}$ & $3.5 \times 10^{-2}$ & $2.0 \times 10^{0}$ \\
\hline 1968 & $1.0 \times 10^{-3}$ & $5.7 \times 10^{-3}$ & $5.0 \times 10^{-4}$ & $7.2 \times 10^{-3}$ & $2.3 \times 10^{-2}$ & $1.3 \times 10^{0}$ \\
\hline 1969 & $5.6 \times 10^{-1}$ & $6.0 \times 10^{-2}$ & $4.6 \times 10^{-4}$ & $6.2 \times 10^{-1}$ & $2.0 \times 10^{0}$ & $1.2 \times 10^{2}$ \\
\hline 1970 & $2.1 \times 10^{-2}$ & $9.2 \times 10^{-3}$ & $3.0 \times 10^{-4}$ & $3.0 \times 10^{-2}$ & $9.7 \times 10^{-2}$ & $5.7 \times 10^{0}$ \\
\hline 1971 & $2.1 \times 10^{-2}$ & $8.4 \times 10^{-3}$ & $5.0 \times 10^{-4}$ & $3.0 \times 10^{-2}$ & $9.7 \times 10^{-2}$ & $5.7 \times 10^{0}$ \\
\hline 1972 & $1.7 \times 10^{-2}$ & $3.8 \times 10^{-3}$ & & $2.0 \times 10^{-2}$ & $6.5 \times 10^{-2}$ & $3.8 \times 10^{0}$ \\
\hline 1973 & $2.2 \times 10^{-2}$ & $1.4 \times 10^{-3}$ & $2.8 \times 10^{-5}$ & $2.3 \times 10^{-2}$ & $7.5 \times 10^{-2}$ & $4.4 \times 10^{0}$ \\
\hline 1974 & $4.7 \times 10^{-3}$ & $2.9 \times 10^{-3}$ & $7.4 \times 10^{-5}$ & $7.7 \times 10^{-3}$ & $2.5 \times 10^{-2}$ & $1.4 \times 10^{0}$ \\
\hline 1975 & $2.0 \times 10^{-3}$ & $5.2 \times 10^{-4}$ & $2.4 \times 10^{-5}$ & $2.5 \times 10^{-3}$ & $8.1 \times 10^{-3}$ & $4.7 \times 10^{-1}$ \\
\hline 1976 & $1.6 \times 10^{-2}$ & $2.4 \times 10^{-4}$ & $2.0 \times 10^{-5}$ & $1.6 \times 10^{-2}$ & $5.3 \times 10^{-2}$ & $3.1 \times 10^{0}$ \\
\hline 1977 & $5.0 \times 10^{-3}$ & $1.0 \times 10^{-4}$ & $2.5 \times 10^{-5}$ & $5.1 \times 10^{-3}$ & $1.6 \times 10^{-2}$ & $9.6 \times 10^{-1}$ \\
\hline 1978 & $6.9 \times 10^{-3}$ & $2.3 \times 10^{-4}$ & $1.7 \times 10^{-5}$ & $7.2 \times 10^{-3}$ & $2.3 \times 10^{-2}$ & $1.3 \times 10^{0}$ \\
\hline 1979 & $1.4 \times 10^{-3}$ & $4.0 \times 10^{-4}$ & $1.2 \times 10^{-5}$ & $1.8 \times 10^{-3}$ & $5.7 \times 10^{-3}$ & $3.3 \times 10^{-1}$ \\
\hline 1980 & $2.8 \times 10^{-3}$ & $1.2 \times 10^{-3}$ & $1.9 \times 10^{-5}$ & $4.0 \times 10^{-3}$ & $1.3 \times 10^{-2}$ & $7.4 \times 10^{-1}$ \\
\hline 1981 & $4.6 \times 10^{-3}$ & $2.8 \times 10^{-3}$ & $9.4 \times 10-6$ & $7.4 \times 10^{-3}$ & $2.4 \times 10^{-2}$ & $1.4 \times 10^{0}$ \\
\hline 1982 & $4.0 \times 10^{-3}$ & $1.7 \times 10^{-3}$ & $1.1 \times 10^{-5}$ & $5.7 \times 10^{-3}$ & $1.8 \times 10^{-2}$ & $1.1 \times 10^{0}$ \\
\hline 1983 & $2.6 \times 10^{-3}$ & $7.2 \times 10^{-4}$ & $1.3 \times 10^{-5}$ & $3.3 \times 10^{-3}$ & $1.1 \times 10^{-2}$ & $6.2 \times 10^{-1}$ \\
\hline 1984 & $1.4 \times 10^{-3}$ & $4.6 \times 10^{-4}$ & $1.2 \times 10^{-5}$ & $1.9 \times 10^{-3}$ & $6.0 \times 10^{-3}$ & $3.5 \times 10^{-1}$ \\
\hline 1985 & $5.4 \times 10^{-4}$ & $5.0 \times 10^{-4}$ & $1.4 \times 10^{-5}$ & $1.1 \times 10^{-3}$ & $3.4 \times 10^{-3}$ & $2.0 \times 10^{-1}$ \\
\hline 1986 & $2.0 \times 10^{-3}$ & $2.5 \times 10^{-4}$ & $8.4 \times 10^{-5}$ & $2.4 \times 10^{-3}$ & $7.6 \times 10^{-3}$ & $4.4 \times 10^{-1}$ \\
\hline 1987 & $2.0 \times 10^{-3}$ & $2.7 \times 10^{-4}$ & $1.3 \times 10^{-4}$ & $2.4 \times 10^{-3}$ & $7.6 \times 10^{-3}$ & $4.4 \times 10^{-1}$ \\
\hline 1988 & $8.3 \times 10^{-4}$ & $3.3 \times 10^{-4}$ & $4.1 \times 10^{-4}$ & $1.6 \times 10^{-3}$ & $5.0 \times 10^{-3}$ & $2.9 \times 10^{-1}$ \\
\hline 1989 & $8.6 \times 10^{-4}$ & $6.8 \times 10^{-4}$ & $1.0 \times 10^{-3}$ & $2.5 \times 10^{-3}$ & $8.2 \times 10^{-3}$ & $4.8 \times 10^{-1}$ \\
\hline 1990 & $3.3 \times 10^{-4}$ & $3.0 \times 10^{-3}$ & $a$ & $3.3 \times 10^{-3}$ & $1.1 \times 10^{-2}$ & $6.3 \times 10^{-1}$ \\
\hline 1991 & $2.6 \times 10^{-4}$ & $4.7 \times 10^{-4}$ & $a$ & $7.4 \times 10^{-4}$ & $2.4 \times 10^{-3}$ & $1.4 \times 10^{-1}$ \\
\hline 1992 & $4.5 \times 10^{-4}$ & $7.5 \times 10^{-4}$ & $a$ & $1.2 \times 10^{-3}$ & $3.8 \times 10^{-3}$ & $2.2 \times 10^{-1}$ \\
\hline 1993 & $1.2 \times 10^{-3}$ & $1.1 \times 10^{-3}$ & $a$ & $2.3 \times 10^{-3}$ & $7.4 \times 10^{-3}$ & $4.3 \times 10^{-1}$ \\
\hline 1994 & $1.6 \times 10^{-3}$ & $7.6 \times 10^{-4}$ & $a$ & $2.4 \times 10^{-3}$ & $7.6 \times 10^{-3}$ & $4.4 \times 10^{-1}$ \\
\hline 1995 & $5.9 \times 10^{-4}$ & $7.3 \times 10^{-4}$ & $\mathbf{a}$ & $1.3 \times 10^{-3}$ & $4.2 \times 10^{-3}$ & $2.5 \times 10^{-1}$ \\
\hline 1996 & $4.8 \times 10^{-4}$ & $5.6 \times 10^{-4}$ & $a$ & $1.0 \times 10^{-3}$ & $3.3 \times 10^{-3}$ & $1.9 \times 10^{-1}$ \\
\hline Total & $7.0 \times 10^{-1}$ & $3.0 \times 10^{0}$ & $7.8 \times 10^{-2}$ & $3.8 \times 10^{0}$ & $1.2 \times 10^{1}$ & $7.1 \times 10^{2}$ \\
\hline
\end{tabular}


Table 4-16. Liquid Plutonium Releases and Dose

\begin{tabular}{|c|c|c|c|c|c|c|c|c|c|c|c|}
\hline Year & $\begin{array}{l}\text { River } \\
\text { Flow } \\
\text { Rate } \\
\left(\mathrm{m}^{3} / \mathrm{s}\right)\end{array}$ & $\begin{array}{c}\text { BJ } \\
\text { Derived } \\
\text { Flow } \\
\text { Rate } \\
\left(\mathrm{m}^{3} / \mathrm{s}\right)\end{array}$ & $\begin{array}{c}\text { PW } \\
\text { Derived } \\
\text { Flow } \\
\text { Rate } \\
\left(\mathrm{m}^{3} / \mathrm{s}\right)\end{array}$ & $\begin{array}{l}\text { Releases } \\
\text { to } \\
\text { Streams } \\
(\mathrm{Ci})^{\mathrm{a}}\end{array}$ & $\begin{array}{l}\text { Max } \\
\text { Ind } \\
\text { Below } \\
\text { SRS } \\
\text { (mrem) }\end{array}$ & $\begin{array}{l}\text { Max } \\
\text { Ind } \\
\text { BJ } \\
\text { (mrem) }\end{array}$ & $\begin{array}{c}\text { Max } \\
\text { Ind } \\
\text { PW } \\
\text { (mrem) }\end{array}$ & $\begin{array}{c}\text { Pop } \\
\text { Dose } \\
\text { BJ } \\
\text { (per-rem) }\end{array}$ & $\begin{array}{c}\text { Pop } \\
\text { Dose } \\
\text { PW } \\
\text { (per-rem) }\end{array}$ & $\begin{array}{c}\text { Pop } \\
\text { Dose } \\
80 \mathrm{~km} \\
\text { (per-rem) }\end{array}$ & $\begin{array}{c}\text { Pop } \\
\text { Dose } \\
\text { Total } \\
\text { (per-rem) }\end{array}$ \\
\hline 1954 & 209 & & 263 & & & & & & & & \\
\hline 1955 & 169 & & 213 & $4.6 \times 10^{-2}$ & $2.9 \times 10^{-2}$ & & $2.2 \times 10^{-2}$ & & $1.7 \times 10^{-1}$ & $8.5 \times 10^{-1}$ & $1.0 \times 10^{0}$ \\
\hline 1956 & 179 & & 225 & $4.2 \times 10^{-2}$ & $2.5 \times 10^{-2}$ & & $1.9 \times 10^{-2}$ & & $1.4 \times 10^{-1}$ & $7.3 \times 10^{-1}$ & $8.8 \times 10^{-1}$ \\
\hline 1959 & 276 & & 348 & $1.3 \times 10^{-2}$ & $5.1 \times 10^{-3}$ & & $3.7 \times 10^{-3}$ & & $2.9 \times 10^{-2}$ & $1.5 \times 10^{-1}$ & $1.8 \times 10^{-1}$ \\
\hline 1960 & 371 & & 468 & $1.7 \times 10^{-2}$ & $4.9 \times 10^{-3}$ & & $3.6 \times 10^{-3}$ & & $2.7 \times 10^{-2}$ & $1.4 \times 10^{-1}$ & $1.7 \times 10^{-1}$ \\
\hline 1961 & 309 & & 389 & $1.7 \times 10^{-2}$ & $5.8 \times 10^{-3}$ & & $4.3 \times 10^{-3}$ & & $3.3 \times 10^{-2}$ & $1.7 \times 10^{-1}$ & $2.0 \times 10^{-1}$ \\
\hline 1962 & 300 & & 377 & $1.2 \times 10^{-2}$ & $4.1 \times 10^{-3}$ & & $3.0 \times 10^{-3}$ & & $2.3 \times 10^{-2}$ & $1.2 \times 10^{-1}$ & $1.4 \times 10^{-1}$ \\
\hline 1963 & 315 & & 397 & $1.6 \times 10^{-2}$ & $5.5 \times 10^{-3}$ & & $4.0 \times 10^{-3}$ & & $3.1 \times 10^{-2}$ & $1.6 \times 10^{-1}$ & $1.9 \times 10^{-1}$ \\
\hline 1964 & 580 & & 731 & $1.5 \times 10^{-2}$ & $2.8 \times 10^{-3}$ & & $2.0 \times 10^{-3}$ & & $1.6 \times 10^{-2}$ & $8.1 \times 10^{-2}$ & $9.6 \times 10^{-2}$ \\
\hline 1969 & 310 & 624 & 390 & $1.1 \times 10^{-2}$ & $3.7 \times 10^{-3}$ & $1.7 \times 10^{-3}$ & $2.7 \times 10^{-3}$ & $4.3 \times 10^{-2}$ & $2.1 \times 10^{-2}$ & $1.1 \times 10^{-1}$ & $1.7 \times 10^{-1}$ \\
\hline 1970 & 232 & 772 & 293 & $2.1 \times 10^{-2}$ & $9.5 \times 10^{-3}$ & $2.6 \times 10^{-3}$ & $7.0 \times 10^{-3}$ & $6.7 \times 10^{-2}$ & $5.4 \times 10^{-2}$ & $2.8 \times 10^{-1}$ & $4.0 \times 10^{-1}$ \\
\hline 1971 & 303 & 1245 & 381 & $4.2 \times 10^{-2}$ & $1.5 \times 10^{-2}$ & $3.3 \times 10^{-3}$ & $1.1 \times 10^{-2}$ & $8.5 \times 10^{-2}$ & $8.4 \times 10^{-2}$ & $4.3 \times 10^{-1}$ & $6.0 \times 10^{-1}$ \\
\hline 1972 & 318 & 775 & 401 & $7.2 \times 10^{-2}$ & $2.4 \times 10^{-2}$ & $9.2 \times 10^{-3}$ & $1.8 \times 10^{-2}$ & $2.3 \times 10^{-1}$ & $1.4 \times 10^{-1}$ & $7.0 \times 10^{-1}$ & $1.1 \times 10^{0}$ \\
\hline 1973 & 409 & 656 & 468 & $4.1 \times 10^{-2}$ & $1.1 \times 10^{-2}$ & $6.1 \times 10^{-3}$ & $8.6 \times 10^{-3}$ & $1.6 \times 10^{-1}$ & $6.6 \times 10^{-2}$ & $3.1 \times 10^{-1}$ & $3 \times 10^{-1}$ \\
\hline 1974 & 314 & 640 & 373 & $1.6 \times 10^{-2}$ & $5.4 \times 10^{-3}$ & $2.4 \times 10^{-3}$ & $4.2 \times 10^{-3}$ & $6.2 \times 10^{-2}$ & $3.2 \times 10^{-2}$ & $1.6 \times 10^{-1}$ & $2.5 \times 10^{-1}$ \\
\hline 1975 & 436 & 877 & 538 & $1.9 \times 10^{-2}$ & $4.6 \times 10^{-3}$ & $2.1 \times 10^{-3}$ & $3.4 \times 10^{-3}$ & $5.3 \times 10^{-2}$ & $2.6 \times 10^{-2}$ & $1.3 \times 10^{-1}$ & $2.1 \times 10^{-1}$ \\
\hline 1976 & 394 & 996 & 472 & $8.3 \times 10^{-3}$ & $2.3 \times 10^{-3}$ & $8.3 \times 10^{-4}$ & $1.7 \times 10^{-3}$ & $2.1 \times 10^{-2}$ & $1.3 \times 10^{-2}$ & $6.6 \times 10^{-2}$ & $1.0 \times 10^{-1}$ \\
\hline 1977 & 330 & 562 & 433 & $8.3 \times 10^{-3}$ & $2.7 \times 10^{-3}$ & $1.5 \times 10^{-3}$ & $1.9 \times 10^{-3}$ & $3.7 \times 10^{-2}$ & $1.5 \times 10^{-2}$ & $7.8 \times 10^{-2}$ & $1.3 \times 10^{-1}$ \\
\hline 1978 & 298 & 689 & 324 & $5.8 \times 10^{-3}$ & $2.1 \times 10^{-3}$ & $8.3 \times 10^{-4}$ & $1.8 \times 10^{-3}$ & $2.1 \times 10^{-2}$ & $1.4 \times 10^{-2}$ & $6.0 \times 10^{-2}$ & $9.5 \times 10^{-2}$ \\
\hline 1979 & 375 & 989 & 495 & $8.8 \times 10^{-3}$ & $2.5 \times 10^{-3}$ & $8.8 \times 10^{-4}$ & $1.8 \times 10^{-3}$ & $2.2 \times 10^{-2}$ & $1.4 \times 10^{-2}$ & $7.3 \times 10^{-2}$ & $1.1 \times 10^{-1}$ \\
\hline 1988 & 152 & 211 & 203 & $5.5 \times 10^{-3}$ & $3.9 \times 10^{-3}$ & $2.6 \times 10^{-3}$ & $2.7 \times 10^{-3}$ & $6.6 \times 10^{-2}$ & $2.1 \times 10^{-2}$ & $1.1 \times 10^{-1}$ & $2.0 \times 10^{-1}$ \\
\hline 1989 & 226 & 240 & 245 & $1.4 \times 10^{-2}$ & $6.7 \times 10^{-3}$ & $5.9 \times 10^{-3}$ & $5.7 \times 10^{-3}$ & $1.5 \times 10^{-1}$ & $4.4 \times 10^{-2}$ & $2.0 \times 10^{-1}$ & $3.9 \times 10^{-1}$ \\
\hline 1990 & 336 & 447 & 367 & $2.3 \times 10^{-2}$ & $7.3 \times 10^{-3}$ & $5.1 \times 10^{-3}$ & $6.2 \times 10^{-3}$ & $1.3 \times 10^{-1}$ & $4.7 \times 10^{-2}$ & $2.1 \times 10^{-1}$ & $3.9 \times 10^{-1}$ \\
\hline 1991 & 328 & 550 & 453 & $2.0 \times 10^{-2}$ & $6.6 \times 10^{-3}$ & $3.6 \times 10^{-3}$ & $4.4 \times 10^{-3}$ & $9.2 \times 10^{-2}$ & $3.4 \times 10^{-2}$ & $1.9 \times 10^{-1}$ & $3.2 \times 10^{-1}$ \\
\hline 1992 & 331 & 350 & 279 & $1.6 \times 10^{-2}$ & $5.2 \times 10^{-3}$ & $4.5 \times 10^{-3}$ & $5.7 \times 10^{-3}$ & $1.2 \times 10^{-1}$ & $4.4 \times 10^{-2}$ & $1.5 \times 10^{-1}$ & $3.1 \times 10^{-1}$ \\
\hline 1993 & 419 & 566 & 416 & $9.6 \times 10^{-3}$ & $2.5 \times 10^{-3}$ & $1.7 \times 10^{-3}$ & $2.3 \times 10^{-3}$ & $4.3 \times 10^{-2}$ & $1.8 \times 10^{-2}$ & $7.1 \times 10^{-2}$ & $1.3 \times 10^{-1}$ \\
\hline 1994 & 347 & 421 & 348 & $1.4 \times 10^{-2}$ & $4.3 \times 10^{-3}$ & $3.3 \times 10^{-3}$ & $4.0 \times 10^{-3}$ & $8.4 \times 10^{-2}$ & $3.1 \times 10^{-2}$ & $1.3 \times 10^{-1}$ & $2.4 \times 10^{-1}$ \\
\hline 1995 & 361 & 438 & 369 & $1.6 \times 10^{-2}$ & $4.8 \times 10^{-3}$ & $3.6 \times 10^{-3}$ & $4.3 \times 10^{-3}$ & $9.2 \times 10^{-2}$ & $3.3 \times 10^{-2}$ & $1.4 \times 10^{-1}$ & $2.6 \times 10^{-1}$ \\
\hline 1996 & 325 & 310 & 287 & $3.0 \times 10^{-2}$ & $9.9 \times 10^{-3}$ & $9.6 \times 10^{-3}$ & $1.0 \times 10^{-2}$ & $2.4 \times 10^{-1}$ & $8.0 \times 10^{-2}$ & $2.9 \times 10^{-1}$ & $6.1 \times 10^{-1}$ \\
\hline Total & & & & $7.4 \times 10^{-1}$ & $2.8 \times 10^{-1}$ & $1.0 \times 10^{-1}$ & $2.1 \times 10^{-1}$ & $2.6 \times 10^{0}$ & $1.6 \times 10^{0}$ & $8.1 \times 10^{0}$ & $1.2 \times 10^{1}$ \\
\hline
\end{tabular}


Table 4-17. Liquid Zr,Nb-95 Releases and Dose

\begin{tabular}{|c|c|c|c|c|c|c|c|c|c|c|c|}
\hline Year & $\begin{array}{c}\text { River } \\
\text { Flow } \\
\text { Rate } \\
\left(\mathrm{m}^{3} / \mathrm{s}\right)\end{array}$ & \begin{tabular}{|c|} 
BJ \\
Derived \\
Flow \\
Rate \\
$\left(\mathrm{m}^{3} / \mathrm{s}\right)$
\end{tabular} & \begin{tabular}{|c|} 
PW \\
Derived \\
Flow \\
Rate \\
$\left(\mathrm{m}^{3} / \mathrm{s}\right)$
\end{tabular} & $\begin{array}{l}\text { Releases } \\
\text { to } \\
\text { Streams } \\
(\mathrm{Ci})^{2}\end{array}$ & $\begin{array}{l}\text { Max } \\
\text { Ind } \\
\text { Below } \\
\text { SRS } \\
\text { (mrem) }\end{array}$ & $\begin{array}{l}\text { Max } \\
\text { Ind } \\
\text { BJ } \\
\text { (mrem) }\end{array}$ & $\begin{array}{c}\text { Max } \\
\text { Ind } \\
\text { PW } \\
\text { (mrem) }\end{array}$ & $\begin{array}{c}\text { Pop } \\
\text { Dose } \\
\text { BJ } \\
\text { (per-rem) }\end{array}$ & $\begin{array}{c}\text { Pop } \\
\text { Dose } \\
\text { PW } \\
\text { (per-rem) }\end{array}$ & $\begin{array}{c}\text { Pop } \\
\text { Dose } \\
80 \mathrm{~km} \\
\text { (per-rem) }\end{array}$ & $\begin{array}{c}\text { Pop } \\
\text { Dose } \\
\text { Total } \\
\text { (per-rem) }\end{array}$ \\
\hline 1954 & 209 & & 263 & & & & & & & & \\
\hline 1955 & 169 & & 213 & & & & & & & & \\
\hline 1956 & 179 & & 225 & & & & & & & & \\
\hline 1957 & 235 & & 297 & & & & & & & & \\
\hline 1958 & 313 & & 394 & & & & & & & & \\
\hline 1959 & 276 & & 348 & & & & & & & & \\
\hline 1960 & 371 & & 468 & $7.5 \times 10^{0}$ & $8.0 \times 10^{-1}$ & & $1.2 \times 10^{-3}$ & & $9.1 \times 10^{-3}$ & $1.3 \times 10^{0}$ & $1.3 \times 10^{0}$ \\
\hline 1961 & 309 & & 389 & $7.0 \times 10^{0}$ & $9.0 \times 10^{-1}$ & & $1.4 \times 10^{-3}$ & & $1.0 \times 10^{-2}$ & $1.5 \times 10^{0}$ & $1.5 \times 10^{0}$ \\
\hline 1962 & 300 & & 377 & $1.7 \times 10^{1}$ & $2.3 \times 10^{0}$ & & $3.5 \times 10^{-3}$ & & $2.6 \times 10^{-2}$ & $3.7 \times 10^{0}$ & $3.8 \times 10^{0}$ \\
\hline 1963 & 315 & & 397 & $3.3 \times 10^{1}$ & $4.1 \times 10^{0}$ & & $6.3 \times 10^{-3}$ & & $4.6 \times 10^{-2}$ & $6.7 \times 10^{0}$ & $6.8 \times 10^{0}$ \\
\hline 1964 & 580 & & 731 & $2.0 \times 10^{1}$ & $1.4 \times 10^{0}$ & & $2.1 \times 10^{-3}$ & & $1.5 \times 10^{-2}$ & $2.2 \times 10^{0}$ & $2.2 \times 10^{0}$ \\
\hline 1965 & 362 & 800 & 456 & $6.2 \times 10^{0}$ & $6.8 \times 10^{-1}$ & $5.9 \times 10^{-4}$ & $1.0 \times 10^{-3}$ & $1.5 \times 10^{-2}$ & $7.7 \times 10^{-3}$ & $1.1 \times 10^{0}$ & $1.1 \times 10^{0}$ \\
\hline 1966 & 316 & 520 & 399 & $8.0 \times 10^{0}$ & $1.0 \times 10^{0}$ & $1.2 \times 10^{-3}$ & $1.5 \times 10^{-3}$ & $2.9 \times 10^{-2}$ & $1.1 \times 10^{-2}$ & $1.6 \times 10^{Q}$ & $1.7 \times 10^{0}$ \\
\hline 1967 & 299 & 625 & 377 & $1.4 \times 10^{1}$ & $1.8 \times 10^{0}$ & $1.7 \times 10^{-3}$ & $2.8 \times 10^{-3}$ & $4.2 \times 10^{-2}$ & $2.1 \times 10^{-2}$ & $3.0 \times 10^{0}$ & $3.1 \times 10^{0}$ \\
\hline 1968 & 273 & 470 & 343 & $8.1 \times 10^{0}$ & $1.2 \times 10^{0}$ & $1.3 \times 10^{-3}$ & $1.8 \times 10^{-3}$ & $3.3 \times 10^{-2}$ & $1.3 \times 10^{-2}$ & $1.9 \times 10^{0}$ & $2.0 \times 10^{0}$ \\
\hline 1969 & 310 & 624 & 390 & $3.6 \times 10^{0}$ & $4.6 \times 10^{-1}$ & $4.4 \times 10^{-4}$ & $7.1 \times 10^{-4}$ & $1.1 \times 10^{-2}$ & $5.2 \times 10^{-3}$ & $7.6 \times 10^{-1}$ & $7.7 \times 10^{-1}$ \\
\hline 1970 & 232 & 772 & 293 & $2.8 \times 10^{0}$ & $4.9 \times 10^{-1}$ & $2.8 \times 10^{-4}$ & $7.4 \times 10^{-4}$ & $7.0 \times 10^{-3}$ & $5.5 \times 10^{-3}$ & $8.0 \times 10^{-1}$ & $8.1 \times 10^{-1}$ \\
\hline 1971 & 303 & 1245 & 381 & $8.9 \times 10^{-1}$ & $1.2 \times 10^{-1}$ & $5.5 \times 10^{-5}$ & $1.8 \times 10^{-4}$ & $1.4 \times 10^{-3}$ & $1.3 \times 10^{-3}$ & $1.9 \times 10^{-1}$ & $1.9 \times 10^{-1}$ \\
\hline 1972 & 318 & 775 & 401 & $9.9 \times 10^{-2}$ & $1.2 \times 10^{-2}$ & $9.8 \times 10^{-6}$ & $1.9 \times 10^{-5}$ & $2.4 \times 10^{-4}$ & $1.4 \times 10^{-4}$ & $2.0 \times 10^{-2}$ & $2.1 \times 10^{-2}$ \\
\hline 1973 & 409 & 656 & 468 & $1.9 \times 10^{-1}$ & $1.9 \times 10^{-2}$ & $2.2 \times 10^{-5}$ & $3.1 \times 10^{-5}$ & $5.6 \times 10^{-4}$ & $2.3 \times 10^{-4}$ & $3.1 \times 10^{-2}$ & $3.1 \times 10^{-2}$ \\
\hline 1974 & 314 & 640 & 373 & $2.3 \times 10^{-1}$ & $2.9 \times 10^{-2}$ & $2.8 \times 10^{-5}$ & $4.8 \times 10^{-5}$ & $6.9 \times 10^{-4}$ & $3.5 \times 10^{-4}$ & $4.8 \times 10^{-2}$ & $5.0 \times 10^{-2}$ \\
\hline 1975 & 436 & 877 & 538 & $3.9 \times 10^{-2}$ & $3.5 \times 10^{-3-}$ & $3.4 \times 10^{-6}$ & $5.5 \times 10^{-6}$ & $8.4 \times 10^{-5}$ & $4.1 \times 10^{-5}$ & $5.8 \times 10^{-3}$ & $5.9 \times 10^{-3}$ \\
\hline 1976 & 394 & 996 & 472 & $1.7 \times 10^{-2}$ & $1.7 \times 10^{-3}$ & $1.3 \times 10^{-6}$ & $2.8 \times 10^{-6}$ & $3.2 \times 10^{-5}$ & $2.0 \times 10^{-5}$ & $2.8 \times 10^{-3}$ & $2.9 \times 10^{-3}$ \\
\hline 1977 & 330 & 562 & 433 & $9.4 \times 10^{-2}$ & $1.1 \times 10^{-2}$ & $1.3 \times 10^{-5}$ & $1.7 \times 10^{-5}$ & $3.2 \times 10^{-4}$ & $1.2 \times 10^{-4}$ & $1.9 \times 10^{-2}$ & $1.9 \times 10^{-2}$ \\
\hline 1978 & 298 & 689 & 324 & $3.5 \times 10^{-4}$ & $4.7 \times 10^{-5}$ & $3.9 \times 10^{-8}$ & $8.2 \times 10^{-8}$ & $9.6 \times 10^{-7}$ & $6.1 \times 10^{-7}$ & $7.6 \times 10^{-5}$ & $7.8 \times 10^{-5}$ \\
\hline 1979 & 375 & 989 & 495 & & & & & & & & \\
\hline 1980 & 374 & 863 & 488 & & & & & & & & \\
\hline 1981 & 187 & 331 & 243 & & & & & & & & \\
\hline 1982 & 203 & 555 & 287 & & & & & & & & \\
\hline 1983 & 350 & 361 & 417 & & & & & & & & \\
\hline 1984 & 361 & 747 & 487 & & & & & & & & \\
\hline 1985 & 203 & 263 & 254 & & & & & & & & \\
\hline 1986 & 175 & 235 & 213 & & & & & & & & \\
\hline 1987 & 254 & 374 & 357 & & & & & & & & \\
\hline 1988 & 152 & 211 & 203 & & & & & & & & \\
\hline 1989 & 226 & 240 & 245 & $5.5 \times 10^{-5}$ & $9.7 \times 10^{-6}$ & $1.8 \times 10^{-8}$ & $1.7 \times 10^{-8}$ & $4.4 \times 10^{-7}$ & $1.3 \times 10^{-7}$ & $1.6 \times 10^{-5}$ & $1.6 \times 10^{-5}$ \\
\hline 1990 & 336 & 447 & 367 & & & & & & & & \\
\hline 1991 & 328 & 550 & 453 & & & & & & & & \\
\hline 1992 & 331 & 350 & 279 & & & & & & & & \\
\hline 1993 & 419 & 566 & 416 & & & & & & & & \\
\hline 1994 & 347 & 421 & 348 & & & & & & & & \\
\hline 1995 & 361 & 438 & 369 & & & & & & & & \\
\hline 1996 & 325 & 310 & 287 & & & & & & & & \\
\hline Total & & & & $1.3 \times 10^{2}$ & $1.5 \times 10^{1}$ & $5.6 \times 10^{-3}$ & $2.3 \times 10^{-2}$ & $1.4 \times 10^{-1}$ & $1.7 \times 10^{-1}$ & $2.5 \times 10^{1}$ & $2.5 \times 10^{1}$ \\
\hline
\end{tabular}


Table 4-18. Atmospheric Ru-106 Releases and Dose

\begin{tabular}{|c|c|c|c|}
\hline Year & $\begin{array}{l}\text { Release } \\
\text { (Ci) }\end{array}$ & $\begin{array}{c}\text { Maximum Individual } \\
\text { Dose at Boundary } \\
\text { (mrem) }\end{array}$ & $\begin{array}{l}\text { Population Dose } \\
\text { (per-rem) }\end{array}$ \\
\hline \multicolumn{4}{|l|}{1954} \\
\hline 1955 & $2.5 \times 10^{1}$ & $8.0 \times 10^{-1}$ & $2.2 \times 10^{1}$ \\
\hline 1956 & $6.3 \times 10^{0}$ & $2.0 \times 10^{-1}$ & $5.4 \times 10^{0}$ \\
\hline 1957 & $1.2 \times 10^{0}$ & $3.9 \times 10^{-2}$ & $1.0 \times 10^{0}$ \\
\hline 1958 & $6.2 \times 10^{-1}$ & $2.0 \times 10^{-2}$ & $5.3 \times 10^{-1}$ \\
\hline 1959 & $1.0 \times 10^{1}$ & $3.2 \times 10^{-1}$ & $8.5 \times 10^{0}$ \\
\hline 1960 & $8.9 \times 10^{\circ}$ & $2.8 \times 10^{-1}$ & $7.5 \times 10^{0}$ \\
\hline 1961 & $3.9 \times 10^{0}$ & $1.2 \times 10^{-1}$ & $3.3 \times 10^{0}$ \\
\hline 1962 & $2.8 \times 10^{0}$ & $8.7 \times 10^{-2}$ & $2.3 \times 10^{0}$ \\
\hline 1963 & $3.9 \times 10^{0}$ & $1.2 \times 10^{-1}$ & $3.3 \times 10^{0}$ \\
\hline 1964 & $2.6 \times 10^{0}$ & $8.2 \times 10^{-2}$ & $2.2 \times 10^{0}$ \\
\hline 1965 & $2.8 \times 10^{0}$ & $8.8 \times 10^{-2}$ & $2.4 \times 10^{0}$ \\
\hline 1966 & $4.6 \times 10^{0}$ & $1.5 \times 10^{-1}$ & $3.9 \times 10^{0}$ \\
\hline 1967 & $4.2 \times 10^{-1}$ & $1.3 \times 10^{-2}$ & $3.6 \times 10^{-1}$ \\
\hline 1968 & $2.0 \times 10^{1}$ & $6.4 \times 10^{-1}$ & $1.7 \times 10^{1}$ \\
\hline 1969 & $4.1 \times 10^{0}$ & $1.3 \times 10^{-1}$ & $3.5 \times 10^{0}$ \\
\hline 1970 & $6.8 \times 10^{-1}$ & $2.1 \times 10^{-2}$ & $5.8 \times 10^{-1}$ \\
\hline 1971 & $2.9 \times 10^{0}$ & $9.2 \times 10^{-2}$ & $2.5 \times 10^{0}$ \\
\hline 1972 & $3.4 \times 10^{0}$ & $1.1 \times 10^{-1}$ & $2.9 \times 10^{0}$ \\
\hline 1973 & $1.3 \times 10^{0}$ & $4.2 \times 10^{-2}$ & $1.1 \times 10^{0}$ \\
\hline 1974 & $1.4 \times 10^{-1}$ & $4.3 \times 10^{-3}$ & $1.2 \times 10^{-1}$ \\
\hline 1975 & $3.7 \times 10^{-2}$ & $1.2 \times 10^{-3}$ & $3.2 \times 10^{-2}$ \\
\hline 1976 & $2.6 \times 10^{-1}$ & $8.3 \times 10^{-3}$ & $2.2 \times 10^{-1}$ \\
\hline 1977 & $1.4 \times 10^{-1}$ & $4.4 \times 10^{-3}$ & $1.2 \times 10^{-1}$ \\
\hline 1978 & $3.2 \times 10^{1}$ & $1.0 \times 10^{0}$ & $2.7 \times 10^{1}$ \\
\hline 1979 & $5.5 \times 10^{-2}$ & $1.7 \times 10^{-3}$ & $4.7 \times 10^{-2}$ \\
\hline 1980 & $6.0 \times 10^{-2}$ & $1.9 \times 10^{-3}$ & $5.1 \times 10^{-2}$ \\
\hline 1981 & $7.9 \times 10^{-2}$ & $2.5 \times 10^{-3}$ & $6.7 \times 10^{-2}$ \\
\hline 1982 & $1.8 \times 10^{-1}$ & $5.8 \times 10^{-3}$ & $1.6 \times 10^{-1}$ \\
\hline 1983 & $8.1 \times 10^{-2}$ & $2.6 \times 10^{-3}$ & $6.9 \times 10^{-2}$ \\
\hline 1984 & $1.5 \times 10^{-1}$ & $4.8 \times 10^{-3}$ & $1.3 \times 10^{-1}$ \\
\hline 1985 & $4.4 \times 10^{-2}$ & $1.4 \times 10^{-3}$ & $3.7 \times 10^{-2}$ \\
\hline 1986 & $5.9 \times 10^{-2}$ & $1.9 \times 10^{-3}$ & $5.0 \times 10^{-2}$ \\
\hline 1987 & $4.5 \times 10^{-2}$ & $1.4 \times 10^{-3}$ & $3.8 \times 10^{-2}$ \\
\hline 1988 & $3.0 \times 10^{-2}$ & $9.5 \times 10^{-4}$ & $2.6 \times 10^{-2}$ \\
\hline 1989 & $3.3 \times 10^{-3}$ & $1.0 \times 10^{-4}$ & $2.8 \times 10^{-3}$ \\
\hline 1990 & $1.2 \times 10^{-3}$ & $3.7 \times 10^{-5}$ & $1.0 \times 10^{-3}$ \\
\hline 1991 & $4.4 \times 10^{-4}$ & $1.4 \times 10^{-5}$ & $3.8 \times 10^{-4}$ \\
\hline 1992 & $1.8 \times 10^{-6}$ & $5.7 \times 10^{-8}$ & $1.5 \times 10^{-6}$ \\
\hline 1993 & $4.0 \times 10^{-6}$ & $1.3 \times 10^{-7}$ & $3.4 \times 10^{-6}$ \\
\hline 1994 & $1.7 \times 10^{-8}$ & $5.3 \times 10^{-1}$ & $1.4 \times 10^{-8}$ \\
\hline 1995 & $1.8 \times 10^{-4}$ & $5.7 \times 10^{-6}$ & $1.5 \times 10^{-4}$ \\
\hline 1996 & $7.0 \times 10^{-2}$ & $2.2 \times 10^{-3}$ & $6.0 \times 10^{-2}$ \\
\hline Total & $1.4 \times 10^{2}$ & $4.4 \times 10^{0}$ & $1.2 \times 10^{2}$ \\
\hline
\end{tabular}


Table 4-19. Liquid Ru-106 Releases and Dose

\begin{tabular}{|c|c|c|c|c|c|c|c|c|c|c|c|}
\hline Year & $\begin{array}{c}\text { River } \\
\text { Flow } \\
\text { Rate } \\
\left(\mathrm{m}^{3} / \mathrm{s}\right)\end{array}$ & \begin{tabular}{|c|} 
BJ \\
Derived \\
Flow \\
Rate \\
$\left(\mathrm{m}^{3} / \mathrm{s}\right)$
\end{tabular} & $\begin{array}{c}\text { PW } \\
\text { Derived } \\
\text { Flow } \\
\text { Rate } \\
\left(\mathrm{m}^{3} / \mathrm{s}\right)\end{array}$ & $\begin{array}{c}\text { Releases } \\
\text { to } \\
\text { Streams } \\
\text { (Ci) }\end{array}$ & $\begin{array}{c}\text { Max } \\
\text { Ind } \\
\text { Below } \\
\text { SRS } \\
\text { (mrem) }\end{array}$ & $\begin{array}{c}\text { Max } \\
\text { Ind } \\
\text { BJ } \\
\text { (mrem) }\end{array}$ & $\begin{array}{c}\text { Max } \\
\text { Ind } \\
\text { PW } \\
\text { (mrem) }\end{array}$ & $\begin{array}{c}\text { Pop } \\
\text { Dose } \\
\text { BJ } \\
\text { (per-rem) }\end{array}$ & $\begin{array}{c}\text { Pop } \\
\text { Dose } \\
\text { PW } \\
\text { (per-rem) }\end{array}$ & $\begin{array}{c}\text { Pop } \\
\text { Dose } \\
80 \mathrm{~km} \\
\text { (per-rem) }\end{array}$ & $\begin{array}{c}\text { Pop } \\
\text { Dose } \\
\text { Total } \\
\text { (per-rem) }\end{array}$ \\
\hline 1954 & 209 & & 263 & $4.5 \times 10^{-2}$ & $1.3 \times 10^{-4}$ & & $8.2 \times 10^{-5}$ & & $6.3 \times 10^{-4}$ & $1.6 \times 10^{-2}$ & $1.7 \times 10^{-2}$ \\
\hline 1955 & 169 & & 213 & $1.7 \times 10^{-1}$ & $6.2 \times 10^{-4}$ & & $3.8 \times 10^{-4}$ & & $2.9 \times 10^{-3}$ & $7.6 \times 10^{-2}$ & $7.9 \times 10^{-2}$ \\
\hline 1956 & 179 & & 225 & $4.6 \times 10^{-1}$ & $1.6 \times 10^{-3}$ & & $9.8 \times 10^{-4}$ & & $7.5 \times 10^{-3}$ & $2.0 \times 10^{-1}$ & $2.0 \times 10^{-1}$ \\
\hline 1957 & 235 & & 297 & $2.2 \times 10^{0}$ & $5.8 \times 10^{-3}$ & & $3.6 \times 10^{-3}$ & & $2.7 \times 10^{-2}$ & $7.1 \times 10^{-1}$ & $7.4 \times 10^{-1}$ \\
\hline 1958 & 313 & & 394 & $8.1 \times 10^{-1}$ & $1.6 \times 10^{-3}$ & & $9.9 \times 10^{-4}$ & & $7.6 \times 10^{-3}$ & $2.0 \times 10^{-1}$ & $2.1 \times 10^{-1}$ \\
\hline 1959 & 276 & & 348 & $2.1 \times 10^{0}$ & $4.7 \times 10^{-3}$ & & $2.9 \times 10^{-3}$ & & $2.2 \times 10^{-2}$ & $5.8 \times 10^{-1}$ & $6.0 \times 10^{-1}$ \\
\hline 1960 & 371 & & 468 & $9.1 \times 10^{0}$ & $1.5 \times 10^{-2}$ & & $9.3 \times 10^{-3}$ & & $7.1 \times 10^{-2}$ & $1.9 \times 10^{0}$ & $1.9 \times 10^{0}$ \\
\hline 1961 & 309 & & 389 & $7.0 \times 10^{0}$ & $1.4 \times 10^{-2}$ & & $8.7 \times 10^{-3}$ & & $6.6 \times 10^{-2}$ & $1.7 \times 10^{0}$ & $1.8 \times 10^{0}$ \\
\hline 1962 & 300 & & 377 & $6.9 \times 10^{0}$ & $1.4 \times 10^{-2}$ & & $8.8 \times 10^{-3}$ & & $6.7 \times 10^{-2}$ & $1.8 \times 10^{0}$ & $1.8 \times 10^{0}$ \\
\hline 1963 & 315 & & 397 & $1.3 \times 10^{1}$ & $2.5 \times 10^{-2}$ & & $1.5 \times 10^{-2}$ & & $1.2 \times 10^{-1}$ & $3.1 \times 10^{0}$ & $3.2 \times 10^{0}$ \\
\hline 1964 & 580 & & 731 & $7.9 \times 10^{0}$ & $8.4 \times 10^{-3}$ & & $5.2 \times 10^{-3}$ & & $4.0 \times 10^{-2}$ & $1.0 \times 10^{0}$ & $1.1 \times 10^{0}$ \\
\hline 1965 & 362 & 800 & 456 & $3.9 \times 10^{0}$ & $6.7 \times 10^{-3}$ & $2.3 \times 10^{-3}$ & $4.1 \times 10^{-3}$ & $5.9 \times 10^{-2}$ & $3.1 \times 10^{-2}$ & $8.2 \times 10^{-1}$ & $9.1 \times 10^{-1}$ \\
\hline 1966 & 316 & 520 & 399 & $1.3 \times 10^{0}$ & $2.5 \times 10^{-3}$ & $1.2 \times 10^{-3}$ & $1.5 \times 10^{-3}$ & $3.0 \times 10^{-2}$ & $1.2 \times 10^{-2}$ & $3.1 \times 10^{-1}$ & $3.5 \times 10^{-1}$ \\
\hline 1967 & 299 & 625 & 377 & $1.9 \times 10^{0}$ & $4.0 \times 10^{-3}$ & $1.5 \times 10^{-3}$ & $2.5 \times 10^{-3}$ & $3.8 \times 10^{-2}$ & $1.9 \times 10^{-2}$ & $5.0 \times 10^{-1}$ & $5.5 \times 10^{-1}$ \\
\hline 1968 & 273 & 470 & 343 & $1.9 \times 10^{0}$ & $4.3 \times 10^{-3}$ & $1.9 \times 10^{-3}$ & $2.7 \times 10^{-3}$ & $4.9 \times 10^{-2}$ & $2.0 \times 10^{-2}$ & $5.3 \times 10^{-1}$ & $6.0 \times 10^{-1}$ \\
\hline 1969 & 310 & 624 & 390 & $5.2 \times 10^{-1}$ & $1.0 \times 10^{-3}$ & $4.0 \times 10^{-4}$ & $6.4 \times 10^{-4}$ & $1.0 \times 10^{-2}$ & $4.9 \times 10^{-3}$ & $1.3 \times 10^{-1}$ & $1.4 \times 10^{-1}$ \\
\hline 1970 & 232 & 772 & 293 & $6.1 \times 10^{-2}$ & $1.6 \times 10^{-4}$ & $3.8 \times 10^{-5}$ & $1.0 \times 10^{-4}$ & $9.6 \times 10^{-4}$ & $7.7 \times 10^{-4}$ & $2.0 \times 10^{-2}$ & $2.2 \times 10^{-2}$ \\
\hline 1971 & 303 & 1245 & 381 & $3.4 \times 10^{-2}$ & $7.1 \times 10^{-5}$ & $1.3 \times 10^{-5}$ & $4.3 \times 10^{-5}$ & $3.4 \times 10^{-4}$ & $3.3 \times 10^{-4}$ & $8.7 \times 10^{-3}$ & $9.4 \times 10^{-3}$ \\
\hline 1972 & 318 & 775 & 401 & $3.9 \times 10^{-2}$ & $7.6 \times 10^{-5}$ & $2.4 \times 10^{-5}$ & $4.7 \times 10^{.5}$ & $6.1 \times 10^{-4}$ & $3.6 \times 10^{-4}$ & $9.4 \times 10^{-3}$ & $1.0 \times 10^{-2}$ \\
\hline 1973 & 409 & 656 & 468 & $4.8 \times 10^{-2}$ & $7.3 \times 10^{-5}$ & $3.5 \times 10^{-5}$ & $4.9 \times 10^{-5}$ & $8.9 \times 10^{-4}$ & $3.8 \times 10^{-4}$ & $9.0 \times 10^{-3}$ & $1.0 \times 10^{-2}$ \\
\hline 1974 & 314 & 640 & 373 & $4.0 \times 10^{-3}$ & $7.9 \times 10^{-6}$ & $3.0 \times 10^{-6}$ & $5.2 \times 10^{-6}$ & $7.6 \times 10^{-5}$ & $3.9 \times 10^{-5}$ & $9.7 \times 10^{-4}$ & $1.1 \times 10^{-3}$ \\
\hline 1975 & 436 & 877 & 538 & $5.4 \times 10^{-2}$ & $7.7 \times 10^{-5}$ & $3.0 \times 10^{-5}$ & $4.8 \times 10^{-5}$ & $7.5 \times 10^{-4}$ & $3.7 \times 10^{-4}$ & $9.5 \times 10^{-3}$ & $1.1 \times 10^{-2}$ \\
\hline 1976 & 394 & 996 & 472 & $1.0 \times 10^{-3}$ & $1.6 \times 10^{-6}$ & $4.8 \times 10^{-7}$ & $1.0 \times 10^{-6}$ & $1.2 \times 10^{-5}$ & $7.8 \times 10^{-6}$ & $1.9 \times 10^{-4}$ & $2.1 \times 10^{-4}$ \\
\hline 1977 & 330 & 562 & 433 & $1.0 \times 10^{-3}$ & $1.9 \times 10^{-6}$ & $8.8 \times 10^{-7}$ & $1.1 \times 10^{-6}$ & $2.2 \times 10^{-5}$ & $8.7 \times 10^{-6}$ & $2.4 \times 10^{-4}$ & $2.7 \times 10^{-4}$ \\
\hline 1978 & 298 & 689 & 324 & & & & & & & & \\
\hline 1979 & 375 & 989 & 495 & & & & & & & & \\
\hline 1980 & 374 & 863 & 488 & & & & & & & & \\
\hline 1981 & 187 & 331 & 243 & & & & & & & & \\
\hline 1982 & 203 & 555 & 287 & & & & & & & & \\
\hline 1983 & 350 & 361 & 417 & & & & & & & & \\
\hline 1984 & 361 & 747 & 487 & & & & & & & & \\
\hline 1985 & 203 & 263 & 254 & & & & & & & & \\
\hline 1986 & 175 & 235 & 213 & & & & & & & & \\
\hline 1987 & 254 & 374 & 357 & & & & & & & & \\
\hline 1988 & 152 & 211 & 203 & & & & & & & & \\
\hline 1989 & 226 & 240 & 245 & $4.7 \times 10^{-3}$ & $1.3 \times 10^{-5}$ & $9.4 \times 10^{-6}$ & $9.2 \times 10^{-6}$ & $2.4 \times 10^{-4}$ & $7.1 \times 10^{-5}$ & $1.6 \times 10^{-3}$ & $1.9 \times 10^{-3}$ \\
\hline 1990 & 336 & 447 & 367 & & & & & & & & \\
\hline 1991 & 328 & 550 & 453 & & & & & & & & \\
\hline 1992 & 331 & 350 & 279 & & & & & & & & \\
\hline 1993 & 419 & 566 & 416 & & & & & & & & \\
\hline 1994 & 347 & 421 & 348 & & & & & & & & \\
\hline 1995 & 361 & 438 & 369 & & & & & & & & \\
\hline 1996 & 325 & 310 & 287 & & & & & & & & \\
\hline Total & & & & $5.9 \times 10^{1}$ & $1.1 \times 10^{-1}$ & $7.5 \times 10^{-3}$ & $6.8 \times 10^{-2}$ & $1.9 \times 10^{-1}$ & $5.2 \times 10^{-1}$ & $1.4 \times 10^{1}$ & $1.4 \times 10^{1}$ \\
\hline
\end{tabular}


Chapter 4. Releases and Doses

Table 4-20. Liquid Ce-144 Releases and Dose

\begin{tabular}{|c|c|c|c|c|c|c|c|c|c|c|c|}
\hline Year & $\begin{array}{l}\text { River } \\
\text { Flow } \\
\text { Rate } \\
\left(\mathrm{m}^{3} / \mathrm{s}\right)\end{array}$ & $\begin{array}{c}\text { BJ } \\
\text { Derived } \\
\text { Flow } \\
\text { Rate } \\
\left(\mathrm{m}^{3} / \mathrm{s}\right)\end{array}$ & $\begin{array}{c}\text { PW } \\
\text { Derived } \\
\text { Flow } \\
\text { Rate } \\
\left(\mathrm{m}^{3} / \mathrm{s}\right)\end{array}$ & $\begin{array}{c}\text { Releases } \\
\text { to } \\
\text { Streams } \\
\text { (Ci) }\end{array}$ & $\begin{array}{c}\text { Max } \\
\text { ind } \\
\text { Below } \\
\text { SRS } \\
\text { (mrem) }\end{array}$ & $\begin{array}{c}\text { Max } \\
\text { Ind } \\
\text { BJ } \\
\text { (mrem) }\end{array}$ & $\begin{array}{c}\text { Max } \\
\text { Ind } \\
\text { PW } \\
\text { (mrem) }\end{array}$ & $\begin{array}{c}\text { Pop } \\
\text { Dose } \\
\text { BJ } \\
\text { (per-rem) } \\
\end{array}$ & $\begin{array}{c}\text { Pop } \\
\text { Dose } \\
\text { PW } \\
\text { (per-rem) }\end{array}$ & $\begin{array}{c}\text { Pop } \\
\text { Dose } \\
80 \mathrm{~km} \\
\text { (per-rem) }\end{array}$ & $\begin{array}{c}\text { Pop } \\
\text { Dose } \\
\text { Total } \\
\text { (per-rem) }\end{array}$ \\
\hline 1954 & 209 & & 263 & & & & & & & & \\
\hline 1955 & 169 & & 213 & & & & & & & & \\
\hline 1956 & 179 & & 225 & & & & & & & & \\
\hline 1957 & 235 & & 297 & & & & & & & & \\
\hline 1958 & 313 & & 394 & & & & & & & & \\
\hline 1959 & 276 & & 348 & & & & & & & & \\
\hline 1960 & 371 & & 468 & $4.0 \times 10^{1}$ & $5.2 \times 10^{-2}$ & & $3.9 \times 10^{-2}$ & & $2.9 \times 10^{-1}$ & $4.6 \times 10^{0}$ & $4.9 \times 10^{0}$ \\
\hline 1961 & 309 & & 389 & $3.6 \times 10^{1}$ & $5.6 \times 10^{-2}$ & & $4.2 \times 10^{-2}$ & & $3.1 \times 10^{-1}$ & $4.9 \times 10^{0}$ & $5.2 \times 10^{0}$ \\
\hline 1962 & 300 & & 377 & $2.3 \times 10^{1}$ & $3.7 \times 10^{-2}$ & & $2.7 \times 10^{-2}$ & & $2.1 \times 10^{-1}$ & $3.2 \times 10^{0}$ & $3.4 \times 10^{0}$ \\
\hline 1963 & 315 & & 397 & $5.1 \times 10^{1}$ & $7.7 \times 10^{-2}$ & & $5.8 \times 10^{-2}$ & & $4.3 \times 10^{-1}$ & $6.8 \times 10^{0}$ & $7.3 \times 10^{0}$ \\
\hline 1964 & 580 & & 731 & $8.8 \times 10^{1}$ & $7.3 \times 10^{-2}$ & & $5.4 \times 10^{-2}$ & & $4.1 \times 10^{-1}$ & $6.4 \times 10^{0}$ & $6.8 \times 10^{0}$ \\
\hline 1965 & 362 & 800 & 456 & $3.3 \times 10^{1}$ & $4.3 \times 10^{-2}$ & $1.8 \times 10^{-2}$ & $3.2 \times 10^{-2}$ & $4.7 \times 10^{-1}$ & $2.4 \times 10^{-1}$ & $3.8 \times 10^{\circ}$ & $4.5 \times 10^{0}$ \\
\hline 1966 & 316 & 520 & 399 & $2.0 \times 10^{1}$ & $3.1 \times 10^{-2}$ & $1.8 \times 10^{-2}$ & $2.3 \times 10^{-2}$ & $4.5 \times 10^{-1}$ & $1.7 \times 10^{-1}$ & $2.7 \times 10^{0}$ & $3.3 \times 10^{0}$ \\
\hline 1967 & 299 & 625 & 377 & $1.7 \times 10^{1}$ & $2.7 \times 10^{-2}$ & $1.2 \times 10^{-2}$ & $2.0 \times 10^{-2}$ & $3.1 \times 10^{-1}$ & $1.5 \times 10^{-1}$ & $2.4 \times 10^{0}$ & $2.8 \times 10^{0}$ \\
\hline 1968 & 273 & 470 & 343 & $3.4 \times 10^{1}$ & $6.0 \times 10^{-2}$ & $3.3 \times 10^{-2}$ & $4.5 \times 10^{-2}$ & $8.4 \times 10^{-1}$ & $3.4 \times 10^{-1}$ & $5.3 \bar{x} 10^{\circ}$ & $6.5 \times 10^{0}$ \\
\hline 1969 & 310 & 624 & 390 & $5.5 \times 10^{0}$ & $8.5 \times 10^{-3}$ & $4.0 \times 10^{-3}$ & $6.4 \times 10^{-3}$ & $1.0 \times 10^{-1}$ & $4.8 \times 10^{-2}$ & $7.5 \times 10^{-1}$ & $9.0 \times 10^{-1}$ \\
\hline 1970 & 232 & 772 & 293 & $2.7 \times 10^{0}$ & $5.6 \times 10^{-3}$ & $1.6 \times 10^{-3}$ & $4.2 \times 10^{-3}$ & $4.0 \times 10^{-2}$ & $3.1 \times 10^{-2}$ & $4.9 \times 10^{-1}$ & $5.6 \times 10^{-1}$ \\
\hline 1971 & 303 & 1245 & 381 & $2.4 \times 10^{0}$ & $3.9 \times 10^{-3}$ & $8.8 \times 10^{-4}$ & $2.9 \times 10^{-3}$ & $2.3 \times 10^{-2}$ & $2.2 \times 10^{-2}$ & $3.4 \times 10^{-1}$ & $3.9 \times 10^{-1}$ \\
\hline 1972 & 318 & 775 & 401 & $4.2 \times 10^{-2}$ & $6.4 \times 10^{-5}$ & $2.5 \times 10^{-5}$ & $4.7 \times 10^{-5}$ & $6.3 \times 10^{-4}$ & $3.6 \times 10^{-4}$ & $5.6 \times 10^{-3}$ & $6.6 \times 10^{-3}$ \\
\hline 1973 & 409 & 656 & 468 & $1.5 \times 10^{-1}$ & $1.7 \times 10^{-4}$ & $1.0 \times 10^{-4}$ & $1.4 \times 10^{-4}$ & $2.6 \times 10^{-3}$ & $1.1 \times 10^{-3}$ & $1.5 \times 10^{-2}$ & $1.9 \times 10^{-2}$ \\
\hline 1974 & 314 & 640 & 373 & $1.5 \times 10^{-1}$ & $2.3 \times 10^{-4}$ & $1.1 \times 10^{-4}$ & $1.9 \times 10^{-4}$ & $2.8 \times 10^{-3}$ & $1.4 \times 10^{-3}$ & $2.1 \times 10^{-2}$ & $2.5 \times 10^{-2}$ \\
\hline 1975 & 436 & 877 & 538 & $3.9 \times 10^{-2}$ & $4.3 \times 10^{-5}$ & $2.0 \times 10^{-5}$ & $3.3 \times 10^{-5}$ & $5.2 \times 10^{-4}$ & $2.5 \times 10^{-4}$ & $3.8 \times 10^{-3}$ & $4.6 \times 10^{-3}$ \\
\hline 1976 & 394 & 996 & 472 & $9.0 \times 10^{-3}$ & $1.1 \times 10^{-5}$ & $4.1 \times 10^{-6}$ & $8.6 \times 10^{-6}$ & $1.0 \times 10^{-4}$ & $6.5 \times 10^{-5}$ & $9.7 \times 10^{-4}$ & $1.1 \times 10^{-3}$ \\
\hline 1977 & 330 & 562 & 433 & $1.4 \times 10^{-1}$ & $2.1 \times 10^{-4}$ & $1.1 \times 10^{-4}$ & $1.5 \times 10^{-4}$ & $2.9 \times 10^{-3}$ & $1.1 \times 10^{-3}$ & $1.8 \times 10^{-2}$ & $2.2 \times 10^{-2}$ \\
\hline 1978 & 298 & 689 & 324 & $9.6 \times 10^{-4}$ & $1.6 \times 10^{-6}$ & $6.3 \times 10^{-7}$ & $1.3 \times 10^{-6}$ & $1.6 \times 10^{-5}$ & $1.0 \times 10^{-5}$ & $1.4 \times 10^{-4}$ & $1.6 \times 10^{-4}$ \\
\hline 1979 & 375 & 989 & 495 & & & & & & & & \\
\hline 1980 & 374 & 863 & 488 & & & & & & & & \\
\hline 1981 & 187 & 331 & 243 & & & & & & & & \\
\hline 1982 & 203 & 555 & 287 & & & & & & & & \\
\hline 1983 & 350 & 361 & 417 & & & & & & & & \\
\hline 1984 & 361 & 747 & 487 & & & & & & & & \\
\hline 1985 & 203 & 263 & 254 & & & & & & & . & \\
\hline 1986 & 175 & 235 & 213 & & & & & & & & \\
\hline 1987 & 254 & 374 & 357 & & & & & & & & \\
\hline 1988 & 152 & 211 & 203 & & & & & & & & \\
\hline 1989 & 226 & 240 & 245 & $4.5 \times 10^{-5}$ & $9.6 \times 10^{-8}$ & $8.5 \times 10^{-8}$ & $8.3 \times 10^{-8}$ & $2.2 \times 10^{-6}$ & $6.2 \times 10^{-7}$ & $8.5 \times 10^{-6}$ & $1.1 \times 10^{-5}$ \\
\hline 1990 & 336 & 447 & 367 & & & & & & & & \\
\hline 1991 & 328 & 550 & 453 & & & & & & & & \\
\hline 1992 & 331 & 350 & 279 & & & & & & & & \\
\hline 1993 & 419 & 566 & 416 & & & & & & & & \\
\hline 1994 & 347 & 421 & 348 & & & & & & & & \\
\hline 1995 & 361 & 438 & 369 & & & & & & & & \\
\hline 1996 & 325 & 310 & 287 & & & & & & & & \\
\hline Total & & & & $3.5 \times 10^{2}$ & $4.7 \times 10^{-1}$ & $8.8 \times 10^{-2}$ & $3.5 \times 10^{-1}$ & $2.2 \times 10^{0}$ & $2.7 \times 10^{0}$ & $4.2 \times 10^{1}$ & $4.7 \times 10^{1}$ \\
\hline
\end{tabular}


Table 4-21. Atmospheric Strontium Releases and Dose

\begin{tabular}{|c|c|c|c|c|c|c|c|c|}
\hline \multirow[b]{2}{*}{ Year } & \multicolumn{3}{|c|}{ Sr $-89,90$} & \multicolumn{3}{|c|}{ Unidentified Beta-Gamma ${ }^{a}$} & \multicolumn{2}{|c|}{ Total Strontium } \\
\hline & $\begin{array}{l}\text { Release } \\
\text { (Ci) }\end{array}$ & $\begin{array}{c}\text { Max } \\
\text { Ind Dose } \\
\text { at Boundary } \\
\text { (mrem) }\end{array}$ & $\begin{array}{c}\text { Population } \\
\text { Dose } \\
\text { (per-rem) }\end{array}$ & $\begin{array}{c}\text { Release } \\
\text { Dose } \\
\text { (Ci) }\end{array}$ & $\begin{array}{c}\text { Max Ind } \\
\text { Dose at } \\
\text { Boundary } \\
\text { (mrem) }\end{array}$ & $\begin{array}{c}\text { Population } \\
\text { Dose } \\
\text { (per-rem) }\end{array}$ & $\begin{array}{c}\text { Max Ind } \\
\text { Dose at } \\
\text { Boundary } \\
\text { (mrem) }\end{array}$ & $\begin{array}{c}\text { Population } \\
\text { Dose } \\
\text { (per-rem) }\end{array}$ \\
\hline \multicolumn{9}{|l|}{1954} \\
\hline 1955 & $4.3 \times 10^{-1}$ & $2.6 \times 10^{-2}$ & $7.7 \times 10^{-1}$ & & & & $2.6 \times 10^{-2}$ & $7.7 \times 10^{-1}$ \\
\hline 1956 & $5.7 \times 10^{-2}$ & $3.5 \times 10^{-3}$ & $1.0 \times 10^{-1}$ & & & & $3.5 \times 10^{-3}$ & $1.0 \times 10^{-1}$ \\
\hline 1957 & $2.1 \times 10^{-2}$ & $1.3 \times 10^{-3}$ & $3.8 \times 10^{-2}$ & & & & $1.3 \times 10^{-3}$ & $3.8 \times 10^{-2}$ \\
\hline 1958 & $2.4 \times 10^{-2}$ & $1.5 \times 10^{-3}$ & $4.3 \times 10^{-2}$ & $1.0 \times 10^{-2}$ & $4.9 \times 10^{-2}$ & $2.4 \times 10^{-2}$ & $5.0 \times 10^{-2}$ & $6.7 \times 10^{-2}$ \\
\hline 1959 & $3.1 \times 10^{-1}$ & $1.9 \times 10^{-2}$ & $5.7 \times 10^{-1}$ & $4.9 \times 10^{-1}$ & $2.4 \times 10^{0}$ & $1.2 \times 10^{0}$ & $2.4 \times 10^{0}$ & $1.8 \times 10^{\circ}$ \\
\hline 1960 & $5.3 \times 10^{-2}$ & $3.3 \times 10^{-3}$ & $9.5 \times 10^{-2}$ & $8.4 \times 10^{-2}$ & $4.1 \times 10^{-1}$ & $2.0 \times 10^{-1}$ & $4.1 \times 10^{-1}$ & $3.0 \times 10^{-1}$ \\
\hline 1961 & $4.1 \times 10^{-2}$ & $2.5 \times 10^{-3}$ & $7.4 \times 10^{-2}$ & $9.8 \times 10^{-2}$ & $4.8 \times 10^{-1}$ & $2.4 \times 10^{-1}$ & $4.8 \times 10^{-1}$ & $3.1 \times 10^{-1}$ \\
\hline 1962 & $3.3 \times 10^{-2}$ & $2.0 \times 10^{-3}$ & $5.9 \times 10^{-2}$ & $3.1 \times 10^{-1}$ & $1.5 \times 10^{0}$ & $7.5 \times 10^{-1}$ & $1.5 \times 10^{0}$ & $8.1 \times 10^{-1}$ \\
\hline 1963 & $3.5 \times 10^{-2}$ & $2.2 \times 10^{-3}$ & $6.3 \times 10^{-2}$ & $2.2 \times 10^{-1}$ & $1.1 \times 10^{0}$ & $5.4 \times 10^{-1}$ & $1.1 \times 10^{0}$ & $6.0 \times 10^{-1}$ \\
\hline 1964 & $4.0 \times 10^{-2}$ & $2.5 \times 10^{-3}$ & $7.2 \times 10^{-2}$ & $6.2 \times 10^{-2}$ & $3.0 \times 10^{-1}$ & $1.5 \times 10^{-1}$ & $3.0 \times 10^{-1}$ & $2.2 \times 10^{-1}$ \\
\hline 1965 & $2.0 \times 10^{-2}$ & $1.2 \times 10^{-3}$ & $3.6 \times 10^{-2}$ & $9.3 \times 10^{-2}$ & $4.5 \times 10^{-1}$ & $2.2 \times 10^{-1}$ & $4.5 \times 10^{-1}$ & $2.6 \times 10^{-1}$ \\
\hline 1966 & $2.1 \times 10^{-2}$ & $1.3 \times 10^{-3}$ & $3.8 \times 10^{-2}$ & $1.3 \times 10^{-2}$ & $6.3 \times 10^{-2}$ & $3.1 \times 10^{-2}$ & $6.4 \times 10^{-2}$ & $6.9 \times 10^{-2}$ \\
\hline 1967 & $1.4 \times 10^{-2}$ & $8.7 \times 10^{-4}$ & $2.5 \times 10^{-2}$ & $1.4 \times 10^{-2}$ & $6.8 \times 10^{-2}$ & $3.4 \times 10^{-2}$ & $6.9 \times 10^{-2}$ & $5.9 \times 10^{-2}$ \\
\hline 1968 & $3.4 \times 10^{-2}$ & $2.1 \times 10^{-3}$ & $6.1 \times 10^{-2}$ & $4.1 \times 10^{-3}$ & $2.0 \times 10^{-2}$ & $9.9 \times 10^{-3}$ & $2.2 \times 10^{-2}$ & $7.1 \times 10^{-2}$ \\
\hline 1969 & $1.2 \times 10^{-1}$ & $7.1 \times 10^{-3}$ & $2.1 \times 10^{-1}$ & $1.1 \times 10^{-2}$ & $5.4 \times 10^{-2}$ & $2.7 \times 10^{-2}$ & $6.1 \times 10^{-2}$ & $2.3 \times 10^{-1}$ \\
\hline 1970 & $5.6 \times 10^{-2}$ & $3.5 \times 10^{-3}$ & $1.0 \times 10^{-1}$ & $6.5 \times 10^{-3}$ & $3.2 \times 10^{-2}$ & $1.6 \times 10^{-2}$ & $3.5 \times 10^{-2}$ & $1.2 \times 10^{-1}$ \\
\hline 1971 & $3.6 \times 10^{-2}$ & $2.2 \times 10^{-3}$ & $6.5 \times 10^{-2}$ & $1.6 \times 10^{-3}$ & $7.8 \times 10^{-3}$ & $3.9 \times 10^{-3}$ & $1.0 \times 10^{-2}$ & $6.9 \times 10^{-2}$ \\
\hline 1972 & $1.3 \times 10^{-1}$ & $8.3 \times 10^{-3}$ & $2.4 \times 10^{-1}$ & $1.0 \times 10^{-3}$ & $4.9 \times 10^{-3}$ & $2.4 \times 10^{-3}$ & $1.3 \times 10^{-2}$ & $2.4 \times 10^{-1}$ \\
\hline 1973 & $1.4 \times 10^{-2}$ & $8.7 \times 10^{-4}$ & $2.5 \times 10^{-2}$ & $5.7 \times 10^{-4}$ & $2.8 \times 10^{-3}$ & $1.4 \times 10^{-3}$ & $3.7 \times 10^{-3}$ & $2.7 \times 10^{-2}$ \\
\hline 1974 & $1.1 \times 10^{-2}$ & $6.8 \times 10^{-4}$ & $2.0 \times 10^{-2}$ & $2.8 \times 10^{-4}$ & $1.4 \times 10^{-3}$ & $6.7 \times 10^{-4}$ & $2.1 \times 10^{-3}$ & $2.0 \times 10^{-2}$ \\
\hline 1975 & $5.0 \times 10^{-3}$ & $3.1 \times 10^{-4}$ & $9.0 \times 10^{-3}$ & $3.8 \times 10^{-4}$ & $1.9 \times 10^{-3}$ & $9.2 \times 10^{-4}$ & $2.2 \times 10^{-3}$ & $9.9 \times 10^{-3}$ \\
\hline 1976 & $4.6 \times 10^{-3}$ & $2.9 \times 10^{-4}$ & $8.3 \times 10^{-3}$ & $7.1 \times 10^{-4}$ & $3.5 \times 10^{-3}$ & $1.7 \times 10^{-3}$ & $3.8 \times 10^{-3}$ & $1.0 \times 10^{-2}$ \\
\hline 1977 & $4.1 \times 10^{-3}$ & $2.5 \times 10^{-4}$ & $7.4 \times 10^{-3}$ & $4.8 \times 10^{-4}$ & $2.3 \times 10^{-3}$ & $1.2 \times 10^{-3}$ & $2.6 \times 10^{-3}$ & $8.6 \times 10^{-3}$ \\
\hline 1978 & $3.8 \times 10^{-3}$ & $2.4 \times 10^{-4}$ & $6.8 \times 10^{-3}$ & $1.5 \times 10^{-4}$ & $7.3 \times 10^{-4}$ & $3.6 \times 10^{-4}$ & $9.7 \times 10^{-4}$ & $7.2 \times 10^{-3}$ \\
\hline 1979 & $2.5 \times 10^{-3}$ & $1.6 \times 10^{-4}$ & $4.5 \times 10^{-3}$ & $1.6 \times 10^{-4}$ & $7.8 \times 10^{-4}$ & $3.9 \times 10^{-4}$ & $9.4 \times 10^{-4}$ & $4.9 \times 10^{-3}$ \\
\hline 1980 & $2.6 \times 10^{-3}$ & $1.6 \times 10^{-4}$ & $4.7 \times 10^{-3}$ & $1.5 \times 10^{-3}$ & $7.3 \times 10^{-3}$ & $3.6 \times 10^{-3}$ & $7.5 \times 10^{-3}$ & $8.3 \times 10^{-3}$ \\
\hline 1981 & $3.0 \times 10^{-3}$ & $1.9 \times 10^{-4}$ & $5.4 \times 10^{-3}$ & $9.2 \times 10^{-4}$ & $4.5 \times 10^{-3}$ & $2.2 \times 10^{-3}$ & $4.7 \times 10^{-3}$ & $7.6 \times 10^{-3}$ \\
\hline 1982 & $2.3 \times 10^{-3}$ & $1.4 \times 10^{-4}$ & $4.1 \times 10^{-3}$ & $6.5 \times 10^{-4}$ & $3.2 \times 10^{-3}$ & $1.6 \times 10^{-3}$ & $3.3 \times 10^{-3}$ & $5.7 \times 10^{-3}$ \\
\hline 1983 & $2.7 \times 10^{-3}$ & $1.7 \times 10^{-4}$ & $4.9 \times 10^{-3}$ & $1.8 \times 10^{-3}$ & $8.8 \times 10^{-3}$ & $4.3 \times 10^{-3}$ & $9.0 \times 10^{-3}$ & $9.2 \times 10^{-3}$ \\
\hline 1984 & $3.5 \times 10^{-3}$ & $2.2 \times 10^{-4}$ & $6.3 \times 10^{-3}$ & $4.3 \times 10^{-4}$ & $2.1 \times 10^{-3}$ & $1.0 \times 10^{-3}$ & $2.3 \times 10^{-3}$ & $7.3 \times 10^{-3}$ \\
\hline 1985 & $1.8 \times 10^{-3}$ & $1.1 \times 10^{-4}$ & $3.2 \times 10^{-3}$ & $3.3 \times 10^{-4}$ & $1.6 \times 10^{-3}$ & $8.0 \times 10^{-4}$ & $1.7 \times 10^{-3}$ & $4.0 \times 10^{-3}$ \\
\hline 1986 & $9.2 \times 10^{-4}$ & $5.7 \times 10^{-5}$ & $1.7 \times 10^{-3}$ & $9.7 \times 10^{-4}$ & $4.7 \times 10^{-3}$ & $2.3 \times 10^{-3}$ & $4.8 \times 10^{-3}$ & $4.0 \times 10^{-3}$ \\
\hline 1987 & $6.1 \times 10^{-4}$ & $3.8 \times 10^{-5}$ & $1.1 \times 10^{-3}$ & $4.8 \times 10^{-4}$ & $2.3 \times 10^{-3}$ & $1.2 \times 10^{-3}$ & $2.3 \times 10^{-3}$ & $2.3 \times 10^{-3}$ \\
\hline 1988 & $1.0 \times 10^{-3}$ & $6.2 \times 10^{-5}$ & $1.8 \times 10^{-3}$ & $2.0 \times 10^{-3}$ & $9.7 \times 10^{-3}$ & $4.8 \times 10^{-3}$ & $9.8 \times 10^{-3}$ & $6.6 \times 10^{-3}$ \\
\hline 1989 & $5.4 \times 10^{-4}$ & $3.3 \times 10^{-5}$ & $9.7 \times 10^{-4}$ & $3.1 \times 10^{-4}$ & $1.5 \times 10^{-3}$ & $7.5 \times 10^{-4}$ & $1.5 \times 10^{-3}$ & $1.7 \times 10^{-3}$ \\
\hline $1990^{\mathrm{b}}$ & & & & $8.0 \times 10^{-3}$ & $3.9 \times 10^{-2}$ & $1.9 \times 10^{-2}$ & $3.9 \times 10^{-2}$ & $1.9 \times 10^{-2}$ \\
\hline $1991^{b}$ & & & & $4.0 \times 10^{-3}$ & $1.9 \times 10^{-2}$ & $9.6 \times 10^{-3}$ & $1.9 \times 10^{-2}$ & $9.6 \times 10^{-3}$ \\
\hline $1992^{b}$ & & & & $2.0 \times 10^{-3}$ & $9.9 \times 10^{-3}$ & $4.9 \times 10^{-3}$ & $9.9 \times 10^{-3}$ & $4.9 \times 10^{-3}$ \\
\hline $1993^{b}$ & & & & $2.3 \times 10^{-3}$ & $1.1 \times 10^{-2}$ & $5.5 \times 10^{-3}$ & $1.1 \times 10^{-2}$ & $5.5 \times 10^{-3}$ \\
\hline $1994^{b}$ & & & & $2.1 \times 10^{-3}$ & $1.0 \times 10^{-2}$ & $5.1 \times 10^{-3}$ & $1.0 \times 10^{-2}$ & $5.1 \times 10^{-3}$ \\
\hline $1995^{b}$ & & & & $5.5 \times 10^{-3}$ & $2.7 \times 10^{-2}$ & $1.3 \times 10^{-2}$ & $2.7 \times 10^{-2}$ & $1.3 \times 10^{-2}$ \\
\hline $1996^{b}$ & & & & $3.1 \times 10^{-3}$ & $1.5 \times 10^{-2}$ & $7.5 \times 10^{-3}$ & $1.5 \times 10^{-2}$ & $7.5 \times 10^{-3}$ \\
\hline Total & $1.5 \times 10^{0}$ & $9.5 \times 10^{-2}$ & $2.8 \times 10^{0}$ & $1.5 \times 10^{0}$ & $7.1 \times 10^{0}$ & $3.5 \times 10^{0}$ & $7.2 \times 10^{0}$ & $6.3 \times 10^{0}$ \\
\hline $\begin{array}{l}\text { More } \\
\text { was n } \\
\text { b Unide }\end{array}$ & or the & Ifed Be & na was & Area. & was $\mathrm{r}$ & 1 tor $5 r$ & uniden & ta-Gamma \\
\hline
\end{tabular}


Table 4-22. Liquid Sr-90 Releases

\begin{tabular}{|c|c|c|c|c|c|c|c|c|c|c|c|c|c|}
\hline Year & $\begin{array}{c}\text { River } \\
\text { Flow } \\
\text { Rate } \\
\left(\mathrm{m}^{3} / \mathrm{s}\right)\end{array}$ & $\begin{array}{c}\text { BJ } \\
\text { Derived } \\
\text { Flow } \\
\text { Rate } \\
\left(\mathrm{m}^{3} / \mathrm{s}\right)\end{array}$ & $\begin{array}{c}\text { PW } \\
\text { Derived } \\
\text { Flow } \\
\text { Rate } \\
\left(\mathrm{m}^{3} / \mathrm{s}\right)\end{array}$ & $\begin{array}{c}\text { Direct } \\
\text { Release } \\
\text { to } \\
\text { Streams } \\
\text { (Ci) }\end{array}$ & $\begin{array}{l}\text { Unident- } \\
\text { ified } \\
\text { Beta- } \\
\text { Gamma } \\
\text { to } \\
\text { Streams } \\
\text { (Ci) }\end{array}$ & $\begin{array}{c}\text { Total } \\
\text { Assuming } \\
\text { Beta- } \\
\text { Gamma } \\
\text { is Sr-90 } \\
\text { (Ci) }\end{array}$ & $\begin{array}{l}\text { Max } \\
\text { Ind } \\
\text { Below } \\
\text { SRS } \\
\text { (mrem) }\end{array}$ & $\begin{array}{l}\text { Max } \\
\text { Ind } \\
\text { BJ } \\
\text { (mrem) }\end{array}$ & $\begin{array}{c}\text { Max } \\
\text { Ind } \\
\text { PW } \\
\text { (mrem) }\end{array}$ & $\begin{array}{c}\text { Pop } \\
\text { Dose } \\
\text { BJ } \\
\text { (per-rem) }\end{array}$ & $\begin{array}{c}\text { Pop } \\
\text { Dose } \\
\text { PW } \\
\text { (per-rem) }\end{array}$ & $\begin{array}{c}\text { Pop } \\
\text { Dose } \\
80 \mathrm{~km} \\
\text { (per-rem) }\end{array}$ & $\begin{array}{c}\text { Pop } \\
\text { Dose } \\
\text { Total } \\
\text { (per-rem) }\end{array}$ \\
\hline 1954 & 209 & & 263 & $3.9 \times 10^{-2}$ & $8.0 \times 10^{-3}$ & $4.7 \times 10^{-2}$ & $1.2 \times 10^{-3}$ & & $6.1 \times 10^{-4}$ & & $4.1 \times 10^{-3}$ & $3.2 \times 10^{-3}$ & $7.3 \times 10^{-3}$ \\
\hline 1955 & 169 & & 213 & $1.5 \times 10^{-1}$ & $1.3 \times 10^{-1}$ & $2.8 \times 10^{-1}$ & $8.8 \times 10^{-3}$ & & $4.4 \times 10^{-3}$ & & $3.0 \times 10^{-2}$ & $2.4 \times 10^{-2}$ & $5.3 \times 10^{-2}$ \\
\hline 1956 & 179 & & 225 & $3.9 \times 10^{-1}$ & $1.6 \times 10^{0}$ & $2.0 \times 10^{0}$ & $6.0 \times 10^{-2}$ & & $3.0 \times 10^{-2}$ & & $2.0 \times 10^{-1}$ & $1.6 \times 10^{-1}$ & $3.6 \times 10^{-1}$ \\
\hline 1957 & 235 & & 297 & $1.1 \times 10^{1}$ & $3.1 \times 10^{-1}$ & $1.1 \times 10^{1}$ & $2.6 \times 10^{-1}$ & & $1.3 \times 10^{-1}$ & & $8.7 \times 10^{-1}$ & $6.9 \times 10^{-1}$ & $1.6 \times 10^{0}$ \\
\hline 1958 & 313 & & 394 & $7.0 \times 10^{-1}$ & $3.9 \times 10^{-1}$ & $1.1 \times 10^{0}$ & $1.9 \times 10^{-2}$ & & $9.4 \times 10^{-3}$ & & $6.3 \times 10^{-2}$ & $5.0 \times 10^{-2}$ & $1.1 \times 10^{-1}$ \\
\hline 1959 & 276 & & 348 & $1.7 \times 10^{0}$ & $9.4 \times 10^{-1}$ & $2.7 \times 10^{0}$ & $5.2 \times 10^{-2}$ & & $2.6 \times 10^{-2}$ & & $1.8 \times 10^{-1}$ & $1.4 \times 10^{-1}$ & $3.1 \times 10^{-1}$ \\
\hline 1960 & 371 & & 468 & $1.8 \times 10^{1}$ & $8.3 \times 10^{0}$ & $2.7 \times 10^{1}$ & $3.9 \times 10^{-1}$ & & $1.9 \times 10^{-1}$ & & $1.3 \times 10^{0}$ & $1.0 \times 10^{0}$ & $2.3 \times 10^{0}$ \\
\hline 1961 & 309 & & 389 & $4.2 \times 10^{0}$ & $3.2 \times 10^{0}$ & $7.4 \times 10^{0}$ & $1.3 \times 10^{-1}$ & & $6.5 \times 10^{-2}$ & & $4.4 \times 10^{-1}$ & $3.5 \times 10^{-1}$ & $7.9 \times 10^{-1}$ \\
\hline 1962 & 300 & & 377 & $6.6 \times 10^{0}$ & $9.5 \times 10^{-1}$ & $7.5 \times 10^{0}$ & $1.3 \times 10^{-1}$ & & $6.8 \times 10^{-2}$ & & $4.6 \times 10^{-1}$ & $3.6 \times 10^{-1}$ & $8.2 \times 10^{-1}$ \\
\hline 1963 & 315 & & 397 & $1.1 \times 10^{1}$ & $1.4 \times 10^{0}$ & $1.2 \times 10^{1}$ & $2.0 \times 10^{-1}$ & & $1.0 \times 10^{-1}$ & & $6.9 \times 10^{-1}$ & $5.5 \times 10^{-1}$ & $1.2 \times 10^{0}$ \\
\hline 1964 & 580 & & 731 & $9.6 \times 10^{0}$ & $1.6 \times 10^{0}$ & $1.1 \times 10^{1}$ & $1.0 \times 10^{-1}$ & & $5.2 \times 10^{-2}$ & & $3.5 \times 10^{-1}$ & $2.8 \times 10^{-1}$ & $6.3 \times 10^{-1}$ \\
\hline 1965 & 362 & 800 & 456 & $5.0 \times 10^{0}$ & $3.6 \times 10^{0}$ & $8.6 \times 10^{0}$ & $1.3 \times 10^{-1}$ & $3.3 \times 10^{-2}$ & $6.4 \times 10^{-2}$ & $8.2 \times 10^{-1}$ & $4.3 \times 10^{-1}$ & $3.4 \times 10^{-1}$ & $1.6 \times 10^{0}$ \\
\hline 1966 & 316 & 520 & 399 & $4.3 \times 10^{0}$ & $3.0 \times 10^{0}$ & $7.3 \times 10^{0}$ & $1.2 \times 10^{-1}$ & $4.4 \times 10^{-2}$ & $6.2 \times 10^{-2}$ & $1.1 \times 10^{0}$ & $4.2 \times 10^{-1}$ & $3.3 \times 10^{-1}$ & $8 \times 10^{0}$ \\
\hline 1967 & 299 & 625 & 377 & $4.6 \times 10^{0}$ & $1.4 \times 10^{t}$ & $1.9 \times 10^{1}$ & $3.3 \times 10^{-1}$ & $9.3 \times 10^{-2}$ & $1.7 \times 10^{-1}$ & $2.3 \times 10^{0}$ & $1.1 \times 10^{0}$ & $9.0 \times 10^{-1}$ & $4.3 \times 10^{0}$ \\
\hline 1968 & 273 & 470 & 343 & $5.7 \times 10^{0}$ & $7.0 \times 10^{-1}$ & $6.4 \times 10^{0}$ & $1.3 \times 10^{-1}$ & $4.2 \times 10^{-2}$ & $6.3 \times 10^{-2}$ & $1.0 \times 10^{0}$ & $4.3 \times 10^{-1}$ & $3.4 \times 10^{-1}$ & $1.8 \times 10^{0}$ \\
\hline 1969 & 310 & 624 & 390 & $3.8 \times 10^{0}$ & $4.0 \times 10^{-1}$ & $4.2 \times 10^{0}$ & $7.3 \times 10^{-2}$ & $2.1 \times 10^{-2}$ & $3.7 \times 10^{-2}$ & $5.2 \times 10^{-1}$ & $2.5 \times 10^{-1}$ & $2.0 \times 10^{-1}$ & $9.6 \times 10^{-1}$ \\
\hline 1970 & 232 & 772 & 293 & $3.4 \times 10^{0}$ & $1.2 \times 10^{-2}$ & $3.5 \times 10^{0}$ & $8.0 \times 10^{-2}$ & $1.4 \times 10^{-2}$ & $4.0 \times 10^{-2}$ & $3.4 \times 10^{-1}$ & $2.7 \times 10^{-1}$ & $2.2 \times 10^{-1}$ & $8.3 \times 10^{-1}$ \\
\hline 1971 & 303 & 1245 & 381 & $3.0 \times 10^{0}$ & $1.8 \times 10^{-1}$ & $3.1 \times 10^{0}$ & $5.6 \times 10^{-2}$ & $7.8 \times 10^{-3}$ & $2.8 \times 10^{-2}$ & $1.9 \times 10^{-1}$ & $1.9 \times 10^{-1}$ & $1.5 \times 10^{-1}$ & $5.3 \times 10^{-1}$ \\
\hline 1972 & 318 & 775 & 401 & $1.4 \times 10^{0}$ & $3.8 \times 10^{-3}$ & $1.4 \times 10^{0}$ & $2.4 \times 10^{-2}$ & $5.8 \times 10^{-3}$ & $1.2 \times 10^{-2}$ & $1.4 \times 10^{-1}$ & $8.2 \times 10^{-2}$ & $6.5 \times 10^{-2}$ & $2.9 \times 10^{-1}$ \\
\hline 1973 & 409 & 656 & 468 & $1.7 \times 10^{0}$ & $5.3 \times 10^{-3}$ & $1.7 \times 10^{0}$ & $2.2 \times 10^{-2}$ & $8.0 \times 10^{-3}$ & $1.2 \times 10^{-2}$ & $2.0 \times 10^{-1}$ & $8.2 \times 10^{-2}$ & $5.9 \times 10^{-2}$ & $3.4 \times 10^{-1}$ \\
\hline 1974 & 314 & 640 & 373 & $1.3 \times 10^{\circ}$ & $2.6 \times 10^{-2}$ & $1.4 \times 10^{0}$ & $2.3 \times 10^{-2}$ & $6.6 \times 10^{-3}$ & $1.2 \times 10^{-2}$ & $1.6 \times 10^{-1}$ & $8.4 \times 10^{-2}$ & $6.3 \times 10^{-2}$ & $3.1 \times 10^{-1}$ \\
\hline 1975 & 436 & 877 & 538 & $1.2 \times 10^{0}$ & $2.5 \times 10^{-2}$ & $1.2 \times 10^{0}$ & $1.5 \times 10^{-2}$ & $4.3 \times 10^{-3}$ & $7.7 \times 10^{-3}$ & $1.1 \times 10^{-1}$ & $5.2 \times 10^{-2}$ & $4.0 \times 10^{-2}$ & $2.0 \times 10^{-1}$ \\
\hline 1976 & 394 & 996 & 472 & $9.3 \times 10^{-1}$ & $3.7 \times 10^{-2}$ & $9.7 \times 10^{-1}$ & $1.3 \times 10^{-2}$ & $3.0 \times 10^{-3}$ & $6.9 \times 10^{-3}$ & $7.4 \times 10^{-2}$ & $4.7 \times 10^{-2}$ & $3.5 \times 10^{-2}$ & $1.6 \times 10^{-1}$ \\
\hline 1977 & 330 & 562 & 433 & $7.0 \times 10^{-1}$ & $1.4 \times 10^{-2}$ & $7.1 \times 10^{-1}$ & $1.2 \times 10^{-2}$ & $3.9 \times 10^{-3}$ & $5.6 \times 10^{-3}$ & $9.7 \times 10^{-2}$ & $3.8 \times 10^{-2}$ & $3.1 \times 10^{-2}$ & $1.7 \times 10^{-1}$ \\
\hline 1978 & 298 & 689 & 324 & $4.9 \times 10^{-1}$ & $6.8 \times 10^{-3}$ & $4.9 \times 10^{-1}$ & $8.9 \times 10^{-3}$ & $2.2 \times 10^{-3}$ & $5.2 \times 10^{-3}$ & $5.5 \times 10^{-2}$ & $3.5 \times 10^{-2}$ & $2.4 \times 10^{-2}$ & $1.1 \times 10^{-1}$ \\
\hline 1979 & 375 & 989 & 495 & $4.8 \times 10^{-1}$ & $1.7 \times 10^{-2}$ & $5.0 \times 10^{-1}$ & $7.1 \times 10^{-3}$ & $1.6 \times 10^{-3}$ & $3.4 \times 10^{-3}$ & $3.8 \times 10^{-2}$ & $2.3 \times 10^{-2}$ & $1.9 \times 10^{-2}$ & $8.1 \times 10^{-2}$ \\
\hline 1980 & 374 & 863 & 488 & $4.0 \times 10^{-1}$ & $8.3 \times 10^{-3}$ & $4.1 \times 10^{-1}$ & $5.9 \times 10^{-3}$ & $1.5 \times 10^{-3}$ & $2.8 \times 10^{-3}$ & $3.6 \times 10^{-2}$ & $1.9 \times 10^{-2}$ & $1.6 \times 10^{-2}$ & $7.1 \times 10^{-2}$ \\
\hline 1981 & 187 & 331 & 243 & $3.5 \times 10^{-1}$ & $1.1 \times 10^{-2}$ & $3.6 \times 10^{-1}$ & $1.0 \times 10^{-2}$ & $3.4 \times 10^{-3}$ & $5.0 \times 10^{-3}$ & $8.3 \times 10^{-2}$ & $3.4 \times 10^{-2}$ & $2.8 \times 10^{-2}$ & $1.4 \times 10^{-1}$ \\
\hline 1982 & 203 & 555 & 287 & $3.1 \times 10^{-1}$ & $3.2 \times 10^{-3}$ & $3.1 \times 10^{-1}$ & $8.3 \times 10^{-3}$ & $1.8 \times 10^{-3}$ & $3.7 \times 10^{-3}$ & $4.3 \times 10^{-2}$ & $2.5 \times 10^{-2}$ & $2.2 \times 10^{-2}$ & $9.0 \times 10^{-2}$ \\
\hline 1983 & 350 & 361 & 417 & $3.1 \times 10^{-1}$ & $1.4 \times 10^{-2}$ & $3.2 \times 10^{-1}$ & $4.9 \times 10^{-3}$ & $2.8 \times 10^{-3}$ & $2.6 \times 10^{-3}$ & $6.8 \times 10^{-2}$ & $1.8 \times 10^{-2}$ & $1.3 \times 10^{-2}$ & $9.8 \times 10^{-2}$ \\
\hline 1984 & 361 & 747 & 487 & $3.3 \times 10^{-1}$ & $3.8 \times 10^{-2}$ & $3.7 \times 10^{-1}$ & $5.5 \times 10^{-3}$ & $1.5 \times 10^{-3}$ & $2.6 \times 10^{-3}$ & $3.8 \times 10^{-2}$ & $1.7 \times 10^{-2}$ & $1.5 \times 10^{-2}$ & $7.0 \times 10^{-2}$ \\
\hline 1985 & 203 & 263 & 254 & $1.8 \times 10^{-1}$ & $2.7 \times 10^{-2}$ & $2.0 \times 10^{-1}$ & $5.4 \times 10^{-3}$ & $2.4 \times 10^{-3}$ & $2.7 \times 10^{-3}$ & $5.9 \times 10^{-2}$ & $1.8 \times 10^{-2}$ & $1.4 \times 10^{-2}$ & $9.2 \times 10^{-2}$ \\
\hline 1986 & 175 & 235 & 213 & $2.6 \times 10^{-1}$ & $2.1 \times 10^{-2}$ & $2.8 \times 10^{-1}$ & $8.6 \times 10^{-3}$ & $3.7 \times 10^{-3}$ & $4.5 \times 10^{-3}$ & $9.1 \times 10^{-2}$ & $3.0 \times 10^{-2}$ & $2.3 \times 10^{-2}$ & $1.4 \times 10^{-1}$ \\
\hline 1987 & 254 & 374 & 357 & $2.9 \times 10^{-1}$ & $1.6 \times 10^{-3}$ & $2.9 \times 10^{-1}$ & $6.1 \times 10^{-3}$ & $2.4 \times 10^{-3}$ & $2.7 \times 10^{-3}$ & $5.9 \times 10^{-2}$ & $1.8 \times 10^{-2}$ & $1.6 \times 10^{-2}$ & $9.3 \times 10^{-2}$ \\
\hline 1988 & 152 & 211 & 203 & $2.1 \times 10^{-1}$ & $8.2 \times 10^{-4}$ & $2.1 \times 10^{-1}$ & $7.3 \times 10^{-3}$ & $3.1 \times 10^{-3}$ & $3.5 \times 10^{-3}$ & $7.5 \times 10^{-2}$ & $2.3 \times 10^{-2}$ & $2.0 \times 10^{-2}$ & $1.2 \times 10^{-1}$ \\
\hline 1989 & 226 & 240 & 245 & $2.0 \times 10^{-1}$ & $5.1 \times 10^{-2}$ & $2.5 \times 10^{-1}$ & $6.0 \times 10^{-3}$ & $3.3 \times 10^{-3}$ & $3.5 \times 10^{-3}$ & $8.1 \times 10^{-2}$ & $2.4 \times 10^{-2}$ & $1.6 \times 10^{-2}$ & $1.2 \times 10^{-1}$ \\
\hline 1990 & 336 & 447 & 367 & $4.3 \times 10^{-1}$ & $a$ & $4.3 \times 10^{-1}$ & $6.9 \times 10^{-3}$ & $3.0 \times 10^{-3}$ & $4.0 \times 10^{-3}$ & $7.4 \times 10^{-2}$ & $2.7 \times 10^{-2}$ & $1.8 \times 10^{-2}$ & $1.2 \times 10^{-1}$ \\
\hline 1991 & 328 & 550 & 453 & $6.0 \times 10^{-1}$ & $a$ & $6.0 \times 10^{-1}$ & $9.8 \times 10^{-3}$ & $3.4 \times 10^{-3}$ & $4.5 \times 10^{-3}$ & $8.3 \times 10^{-2}$ & $3.0 \times 10^{-2}$ & $2.6 \times 10^{-2}$ & $1.4 \times 10^{-1}$ \\
\hline 1992 & 331 & 350 & 279 & $7.9 \times 10^{-1}$ & $a$ & $7.9 \times 10^{-1}$ & $1.3 \times 10^{-2}$ & $7.0 \times 10^{-3}$ & $9.6 \times 10^{-3}$ & $1.7 \times 10^{-1}$ & $6.5 \times 10^{-2}$ & $3.4 \times 10^{-2}$ & $2.7 \times 10^{-1}$ \\
\hline 1993 & 419 & 566 & 416 & $4.8 \times 10^{-1}$ & $a$ & $4.8 \times 10^{-1}$ & $6.2 \times 10^{-3}$ & $2.6 \times 10^{-3}$ & $3.9 \times 10^{-3}$ & $6.5 \times 10^{-2}$ & $2.6 \times 10^{-2}$ & $1.7 \times 10^{-2}$ & $1.1 \times 10^{-1}$ \\
\hline 1994 & 347 & 421 & 348 & $3.9 \times 10^{-1}$ & $a$ & $3.9 \times 10^{-1}$ & $6.0 \times 10^{-3}$ & $2.9 \times 10^{-3}$ & $3.8 \times 10^{-3}$ & $7.1 \times 10^{-2}$ & $2.6 \times 10^{-2}$ & $1.6 \times 10^{-2}$ & $1.1 \times 10^{-1}$ \\
\hline 1995 & 361 & 438 & 369 & $4.0 \times 10^{-1}$ & $a$ & $4.0 \times 10^{-1}$ & $6.0 \times 10^{-3}$ & $2.8 \times 10^{-3}$ & $3.7 \times 10^{-3}$ & $7.0 \times 10^{-2}$ & $2.5 \times 10^{-2}$ & $1.6 \times 10^{-2}$ & $1.1 \times 10^{-1}$ \\
\hline 1996 & 325 & 310 & 287 & $2.6 \times 10^{-1}$ & $a$ & $2.6 \times 10^{-1}$ & $4.3 \times 10^{-3}$ & $2.6 \times 10^{-3}$ & $3.1 \times 10^{-3}$ & $6.4 \times 10^{-2}$ & $2.1 \times 10^{-2}$ & $1.2 \times 10^{-2}$ & $9.6 \times 10^{-2}$ \\
\hline Total & & & & $1.1 \times 10^{2}$ & $4.5 \times 10^{1}$ & $1.5 \times 10^{2}$ & $2.5 \times 10^{0}$ & $3.4 \times 10^{-1}$ & $1.3 \times 10^{0}$ & $8.3 \times 10^{0}$ & $8.6 \times 10^{0}$ & $6.8 \times 10^{0}$ & $2.4 \times 10^{1}$ \\
\hline
\end{tabular}


Assessment of Radionuclides

in the Savannah River Site Environment-Summary (U)

WSRC-TR-98-00162

Table 4-23. Atmospheric H-3 Releases and Dose

\begin{tabular}{|c|c|c|c|c|}
\hline Year & $\begin{array}{c}\text { Total } \\
\text { Tritium } \\
\text { Reieased }(\mathrm{Ci})\end{array}$ & $\begin{array}{l}\text { Tritium Oxide } \\
\text { (Ci) (a) }\end{array}$ & $\begin{array}{c}\text { Maximum Individual Dose } \\
\text { at Boundary } \\
\text { (mrem) }\end{array}$ & $\begin{array}{l}\text { Population Dose } \\
\text { (per-rem) }\end{array}$ \\
\hline \multicolumn{5}{|l|}{1954} \\
\hline 1955 & $3.6 \times 10^{4}$ & & $2.7 \times 10^{-2}$ & $1.7 \times 10^{0}$ \\
\hline 1956 & $4.7 \times 10^{5}$ & & $3.5 \times 10^{-1}$ & $2.2 \times 10^{1}$ \\
\hline 1957 & $1.2 \times 10^{6}$ & & $8.9 \times 10^{-1}$ & $5.6 \times 10^{1}$ \\
\hline 1958 & $2.4 \times 10^{6}$ & & $1.8 \times 10^{0}$ & $1.1 \times 10^{2}$ \\
\hline 1959 & $1.1 \times 10^{6}$ & & $7.8 \times 10^{-1}$ & $4.9 \times 10^{1}$ \\
\hline 1960 & $9.5 \times 10^{5}$ & . & $7.1 \times 10^{-1}$ & $4.4 \times 10^{1}$ \\
\hline 1961 & $8.9 \times 10^{5}$ & & $6.6 \times 10^{-1}$ & $4.1 \times 10^{1}$ \\
\hline 1962 & $1.1 \times 10^{6}$ & & $8.2 \times 10^{-1}$ & $5.1 \times 10^{1}$ \\
\hline 1963 & $1.1 \times 10^{6}$ & & $8.4 \times 10^{-1}$ & $5.2 \times 10^{1}$ \\
\hline 1964 & $1.5 \times 10^{6}$ & & $1.1 \times 10^{0}$ & $7.0 \times 10^{1}$ \\
\hline 1965 & $7.4 \times 10^{5}$ & & $5.5 \times 10^{-1}$ & $3.4 \times 10^{1}$ \\
\hline 1956 & $6.8 \times 10^{5}$ & & $5.0 \times 10^{-1}$ & $3.1 \times 10^{1}$ \\
\hline 1967 & $6.9 \times 10^{5}$ & & $5.1 \times 10^{-1}$ & $3.2 \times 10^{1}$ \\
\hline 1968 & $7.6 \times 10^{5}$ & & $5.7 \times 10^{-1}$ & $3.5 \times 10^{6}$ \\
\hline 1969 & $5.0 \times 10^{5}$ & & $3.7 \times 10^{-1}$ & $2.3 \times 10^{1-}$ \\
\hline 1970 & $5.1 \times 10^{5}$ & & $3.8 \times 10^{-1}$ & $2.4 \times 10^{1}$ \\
\hline 1971 & $6.2 \times 10^{5}$ & & $4.6 \times 10^{-1}$ & $2.9 \times 10^{1}$ \\
\hline 1972 & $8.2 \times 10^{5}$ & & $6.1 \times 10^{-1}$ & $3.8 \times 10^{1}$ \\
\hline 1973 & $6.0 \times 10^{5}$ & & $4.5 \times 10^{-1}$ & $2.8 \times 10^{1}$ \\
\hline 1974 & $9.4 \times 10^{5}$ & & $7.0 \times 10^{-1}$ & $4.3 \times 10^{1}$ \\
\hline 1975 & $5.2 \times 10^{5}$ & & $3.8 \times 10^{-1}$ & $2.4 \times 10^{1}$ \\
\hline 1976 & $3.0 \times 10^{5}$ & & $2.3 \times 10^{-1}$ & $1.4 \times 10^{1}$ \\
\hline 1977 & $3.8 \times 10^{5}$ & & $2.8 \times 10^{-1}$ & $1.8 \times 10^{1}$ \\
\hline 1978 & $3.6 \times 10^{5}$ & & $2.7 \times 10^{-1}$ & $1.7 \times 10^{1}$ \\
\hline 1979 & $3.3 \times 10^{5}$ & & $2.5 \times 10^{-1}$ & $1.5 \times 10^{1}$ \\
\hline 1980 & $3.2 \times 10^{5}$ & & $2.4 \times 10^{-1}$ & $1.5 \times 10^{1}$ \\
\hline 1981 & $4.0 \times 10^{5}$ & & $2.9 \times 10^{-1}$ & $1.8 \times 10^{1}$ \\
\hline 1982 & $4.3 \times 10^{5}$ & & $3.2 \times 10^{-1}$ & $2.0 \times 10^{1}$ \\
\hline 1983 & $6.2 \times 10^{5}$ & & $4.6 \times 10^{-1}$ & $2.9 \times 10^{1}$ \\
\hline 1984 & $7.9 \times 10^{5}$ & & $5.8 \times 10^{-1}$ & $3.6 \times 10^{1}$ \\
\hline 1985 & $6.7 \times 10^{5}$ & $4.9 \times 10^{5}$ & $3.6 \times 10^{-1}$ & $2.3 \times 10^{1}$ \\
\hline 1986 & $4.3 \times 10^{5}$ & $2.9 \times 10^{5}$ & $2.1 \times 10^{-1}$ & $1.3 \times 10^{1}$ \\
\hline 1987 & $5.9 \times 10^{5}$ & $2.7 \times 10^{5}$ & $2.0 \times 10^{-1}$ & $1.3 \times 10^{1}$ \\
\hline 1988 & $4.6 \times 10^{5}$ & $2.9 \times 10^{5}$ & $2.1 \times 10^{-1}$ & $1.3 \times 10^{1}$ \\
\hline 1989 & $3.1 \times 10^{5}$ & $2.2 \times 10^{5}$ & $1.6 \times 10^{-1}$ & $1.0 \times 10^{1}$ \\
\hline 1990 & $2.5 \times 10^{5}$ & $1.8 \times 10^{5}$ & $1.3 \times 10^{-1}$ & $8.1 \times 10^{0}$ \\
\hline 1991 & $2.0 \times 10^{5}$ & $1.4 \times 10^{5}$ & $1.0 \times 10^{-1}$ & $6.3 \times 10^{0}$ \\
\hline 1992 & $1.6 \times 10^{5}$ & $1.0 \times 10^{5}$ & $7.4 \times 10^{-2}$ & $4.6 \times 10^{0}$ \\
\hline 1993 & $1.9 \times 10^{5}$ & $1.3 \times 10^{5}$ & $9.9 \times 10^{-2}$ & $6.2 \times 10^{0}$ \\
\hline 1994 & $1.6 \times 10^{5}$ & $1.1 \times 10^{5}$ & $7.9 \times 10^{-2}$ & $5.0 \times 10^{0}$ \\
\hline 1995 & $9.7 \times 10^{4}$ & $5.5 \times 10^{4}$ & $4.1 \times 10^{-2}$ & $2.5 \times 10^{0}$ \\
\hline 1996 & $5.5 \times 10^{4}$ & $4.0 \times 10^{4}$ & $3.0 \times 10^{-2}$ & $1.9 \times 10^{0}$ \\
\hline Total & $2.6 \times 10^{7}$ & $2.3 \times 10^{6}$ & $1.8 \times 10^{1}$ & $1.1 \times 10^{3}$ \\
\hline
\end{tabular}


Chapter 4. Releases and Doses

Table 4-24. Liquid H-3 Releases and Dose to the Maximally Exposed Individual

\begin{tabular}{|c|c|c|c|c|c|c|c|c|c|}
\hline \multirow[b]{2}{*}{ Year } & \multirow[b]{2}{*}{$\begin{array}{l}\text { River } \\
\text { Flow } \\
\text { Rate } \\
\left(\mathrm{m}^{3} / \mathrm{s}\right) \\
\end{array}$} & \multirow[b]{2}{*}{$\begin{array}{c}\text { BJ } \\
\text { Derived } \\
\text { Flow } \\
\text { Rate } \\
\left(\mathrm{m}^{3} / \mathrm{s}\right)\end{array}$} & \multirow[b]{2}{*}{$\begin{array}{c}\text { PW } \\
\text { Derived } \\
\text { Flow } \\
\text { Rate } \\
\left(\mathrm{m}^{3} / \mathrm{s}\right)\end{array}$} & \multicolumn{3}{|c|}{ Tritium Releases to Streams } & \multicolumn{3}{|c|}{ Dose to Maximally Exposed Individual } \\
\hline & & & & $\begin{array}{c}\text { Direct } \\
\text { Release } \\
\text { to Streams } \\
(\mathrm{Ci})\end{array}$ & $\begin{array}{l}\text { Migration } \\
\text { to } \\
\text { Streams } \\
\text { (Ci) }\end{array}$ & $\begin{array}{c}\text { Total } \\
\text { Release } \\
\text { (Ci) }\end{array}$ & $\begin{array}{c}\text { Below } \\
\text { SRS } \\
\text { (mrem) }\end{array}$ & $\begin{array}{c}\text { BJ } \\
\text { Water } \\
\text { Treatment } \\
\text { Plant } \\
\text { (mrem) }\end{array}$ & $\begin{array}{c}\text { PW } \\
\text { Water } \\
\text { Treatment } \\
\text { Plant } \\
\text { (mrem) }\end{array}$ \\
\hline 1954 & 209 & & 263 & & & & & & \\
\hline 1955 & 169 & & 213 & $5.9 \times 10^{3}$ & $0.0 \times 10^{0}$ & $5.9 \times 10^{3}$ & $5.2 \times 10^{-2}$ & & $4.0 \times 10^{-2}$ \\
\hline 1956 & 179 & & 225 & $9.4 \times 10^{3}$ & $0.0 \times 10^{0}$ & $9.4 \times 10^{3}$ & $7.9 \times 10^{-2}$ & & $6.0 \times 10^{-2}$ \\
\hline 1957 & 235 & & 297 & $1.5 \times 10^{4}$ & $0.0 \times 10^{0}$ & $1.5 \times 10^{4}$ & $9.6 \times 10^{-2}$ & & $7.3 \times 10^{-2}$ \\
\hline 1958 & 313 & & 394 & $2.4 \times 10^{4}$ & $4.0 \times 10^{2}$ & $2.4 \times 10^{4}$ & $1.2 \times 10^{-1}$ & & $8.9 \times 10^{-2}$ \\
\hline 1959 & 276 & & 348 & $4.1 \times 10^{4}$ & $8.0 \times 10^{2}$ & $4.2 \times 10^{4}$ & $2.3 \times 10^{-1}$ & & $1.7 \times 10^{-1}$ \\
\hline 1960 & 371 & & 468 & $4.7 \times 10^{4}$ & $1.6 \times 10^{3}$ & $4.9 \times 10^{4}$ & $2.0 \times 10^{-1}$ & & $1.5 \times 10^{-1}$ \\
\hline 1961 & 309 & & 389 & $5.4 \times 10^{4}$ & $2.0 \times 10^{3}$ & $5.6 \times 10^{4}$ & $2.7 \times 10^{-1}$ & & $2.1 \times 10^{-1}$ \\
\hline 1962 & 300 & & 377 & $4.7 \times 10^{4}$ & $1.7 \times 10^{3}$ & $4.8 \times 10^{4}$ & $2.4 \times 10^{-1}$ & & $1.9 \times 10^{-1}$ \\
\hline 1963 & 315 & & 397 & $7.5 \times 10^{4}$ & $2.7 \times 10^{3}$ & $7.7 \times 10^{4}$ & $3.7 \times 10^{-1}$ & & $2.8 \times 10^{-1}$ \\
\hline 1964 & 580 & & 731 & $8.1 \times 10^{4}$ & $4.7 \times 10^{3}$ & $8.5 \times 10^{4}$ & $2.2 \times 10^{-1}$ & & $1.7 \times 10^{-1}$ \\
\hline 1965 & 362 & 800 & 456 & $9.8 \times 10^{4}$ & $5.6 \times 10^{3}$ & $1.0 \times 10^{5}$ & $4.3 \times 10^{-1}$ & $1.9 \times 10^{-1}$ & $3.3 \times 10^{-1}$ \\
\hline 1966 & 316 & 520 & 399 & $7.7 \times 10^{4}$ & $4.6 \times 10^{3}$ & $8.2 \times 10^{4}$ & $3.9 \times 10^{-1}$ & $2.3 \times 10^{-1}$ & $3.0 \times 10^{-1}$ \\
\hline 1967 & 299 & 625 & 377 & $6.4 \times 10^{4}$ & $5.6 \times 10^{3}$ & $7.0 \times 10^{4}$ & $3.5 \times 10^{-1}$ & $1.6 \times 10^{-1}$ & $2.7 \times 10^{-1}$ \\
\hline 1968 & 273 & 470 & 343 & $5.6 \times 10^{4}$ & $5.8 \times 10^{3}$ & $6.2 \times 10^{4}$ & $3.4 \times 10^{-1}$ & $1.9 \times 10^{-1}$ & $2.6 \times 10^{-1}$ \\
\hline 1969 & 310 & 624 & 390 & $4.5 \times 10^{4}$ & $1.9 \times 10^{4}$ & $6.4 \times 10^{4}$ & $3.1 \times 10^{-1}$ & $1.5 \times 10^{-1}$ & $2.4 \times 10^{-1}$ \\
\hline 1970 & 232 & 772 & 293 & $2.4 \times 10^{4}$ & $1.3 \times 10^{4}$ & $3.7 \times 10^{4}$ & $2.4 \times 10^{-1}$ & $6.9 \times 10^{-2}$ & $1.8 \times 10^{-1}$ \\
\hline 1971 & 303 & 1245 & 381 & $2.0 \times 10^{4}$ & $1.8 \times 10^{4}$ & $3.8 \times 10^{4}$ & $1.9 \times 10^{-1}$ & $4.4 \times 10^{-2}$ & $1.4 \times 10^{-1}$ \\
\hline 1972 & 318 & 775 & 401 & $2.9 \times 10^{4}$ & $1.7 \times 10^{4}$ & $4.6 \times 10^{4}$ & $2.2 \times 10^{-1}$ & $8.6 \times 10^{-2}$ & $1.7 \times 10^{-1}$ \\
\hline 1973 & 409 & 656 & 468 & $4.3 \times 10^{4}$ & $2.8 \times 10^{4}$ & $7.1 \times 10^{4}$ & $2.6 \times 10^{-1}$ & $1.6 \times 10^{-1}$ & $2.2 \times 10^{-1}$ \\
\hline 1974 & 314 & 640 & 373 & $3.3 \times 10^{4}$ & $2.7 \times 10^{4}$ & $6.0 \times 10^{4}$ & $2.9 \times 10^{-1}$ & $1.4 \times 10^{-1}$ & $2.3 \times 10^{-1}$ \\
\hline 1975 & 436 & 877 & 538 & $2.8 \times 10^{4}$ & $2.8 \times 10^{4}$ & $5.6 \times 10^{4}$ & $1.9 \times 10^{-1}$ & $9.2 \times 10^{-2}$ & $1.5 \times 10^{-1}$ \\
\hline 1976 & 394 & 996 & 472 & $3.2 \times 10^{4}$ & $2.8 \times 10^{4}$ & $6.0 \times 10^{4}$ & $2.3 \times 10^{-1}$ & $8.6 \times 10^{-2}$ & $1.8 \times 10^{-1}$ \\
\hline 1977 & 330 & 562 & 433 & $2.7 \times 10^{4}$ & $1.7 \times 10^{4}$ & $4.4 \times 10^{4}$ & $2.0 \times 10^{-1}$ & $1.1 \times 10^{-1}$ & $1.5 \times 10^{-1}$ \\
\hline 1978 & 298 & 689 & 324 & $1.7 \times 10^{4}$ & $2.0 \times 10^{4}$ & $3.8 \times 10^{4}$ & $1.9 \times 10^{-1}$ & $7.9 \times 10^{-2}$ & $1.7 \times 10^{-1}$ \\
\hline 1979 & 375 & 989 & 495 & $1.0 \times 10^{4}$ & $1.9 \times 10^{4}$ & $2.9 \times 10^{4}$ & $1.2 \times 10^{-1}$ & $4.3 \times 10^{-2}$ & $8.6 \times 10^{-2}$ \\
\hline 1980 & 374 & 863 & 488 & $1.0 \times 10^{4}$ & $1.4 \times 10^{4}$ & $2.5 \times 10^{4}$ & $9.9 \times 10^{-2}$ & $4.1 \times 10^{-2}$ & $7.3 \times 10^{-2}$ \\
\hline 1981 & 187 & 331 & 243 & $9.6 \times 10^{3}$ & $1.4 \times 10^{4}$ & $2.4 \times 10^{4}$ & $1.9 \times 10^{-1}$ & $1.0 \times 10^{-1}$ & $1.4 \times 10^{-1}$ \\
\hline 1982 & 203 & 555 & 287 & $1.1 \times 10^{4}$ & $2.2 \times 10^{4}$ & $3.3 \times 10^{4}$ & $2.4 \times 10^{-1}$ & $8.5 \times 10^{-2}$ & $1.6 \times 10^{-1}$ \\
\hline 1983 & 350 & 361 & 417 & $1.4 \times 10^{4}$ & $2.0 \times 10^{4}$ & $3.4 \times 10^{4}$ & $1.5 \times 10^{-1}$ & $1.4 \times 10^{-1}$ & $1.2 \times 10^{-1}$ \\
\hline 1984 & 361 & 747 & 487 & $1.1 \times 10^{4}$ & $2.2 \times 10^{4}$ & $3.3 \times 10^{4}$ & $1.4 \times 10^{-1}$ & $6.3 \times 10^{-2}$ & $9.7 \times 10^{-2}$ \\
\hline 1985 & 203 & 263 & 254 & $6.1 \times 10^{3}$ & $1.9 \times 10^{4}$ & $2.5 \times 10^{4}$ & $1.8 \times 10^{-1}$ & $1.4 \times 10^{-1}$ & $1.4 \times 10^{-1}$ \\
\hline 1986 & 175 & 235 & 213 & $7.3 \times 10^{3}$ & $2.1 \times 10^{4}$ & $2.8 \times 10^{4}$ & $2.4 \times 10^{-1}$ & $1.7 \times 10^{-1}$ & $1.9 \times 10^{-1}$ \\
\hline 1987 & 254 & 374 & 357 & $4.5 \times 10^{3}$ & $1.8 \times 10^{4}$ & $2.3 \times 10^{4}$ & $1.3 \times 10^{-1}$ & $8.8 \times 10^{-2}$ & $9.2 \times 10^{-2}$ \\
\hline 1988 & 152 & 211 & 203 & $5.4 \times 10^{3}$ & $1.4 \times 10^{4}$ & $1.9 \times 10^{4}$ & $1.9 \times 10^{-1}$ & $1.3 \times 10^{-1}$ & $1.4 \times 10^{-1}$ \\
\hline 1989 & 226 & 240 & 245 & $3.6 \times 10^{3}$ & $1.4 \times 10^{4}$ & $1.7 \times 10^{4}$ & $1.1 \times 10^{-1}$ & $1.0 \times 10^{-1}$ & $1.0 \times 10^{-1}$ \\
\hline 1990 & 336 & 447 & 367 & $2.6 \times 10^{3}$ & $1.4 \times 10^{4}$ & $1.6 \times 10^{4}$ & $7.2 \times 10^{-2}$ & $5.2 \times 10^{-2}$ & $6.4 \times 10^{-2}$ \\
\hline 1991 & 328 & 550 & 453 & $1.1 \times 10^{4}$ & $1.6 \times 10^{4}$ & $2.7 \times 10^{4}$ & $1.3 \times 10^{-1}$ & $7.2 \times 10^{-2}$ & $8.7 \times 10^{-2}$ \\
\hline 1992 & 331 & 350 & 279 & $2.4 \times 10^{3}$ & $1.1 \times 10^{4}$ & $1.4 \times 10^{4}$ & $6.3 \times 10^{-2}$ & $5.8 \times 10^{-2}$ & $7.2 \times 10^{-2}$ \\
\hline 1993 & 419 & 566 & 416 & $1.7 \times 10^{3}$ & $9.6 \times 10^{3}$ & $1.3 \times 10^{4}$ & $4.7 \times 10^{-2}$ & $3.3 \times 10^{-2}$ & $4.5 \times 10^{-2}$ \\
\hline 1994 & 347 & 421 & 348 & $1.2 \times 10^{3}$ & $9.8 \times 10^{3}$ & $1.1 \times 10^{4}$ & $4.8 \times 10^{-2}$ & $3.8 \times 10^{-2}$ & $4.6 \times 10^{-2}$ \\
\hline 1995 & 361 & 438 & 369 & $1.3 \times 10^{3}$ & $9.7 \times 10^{3}$ & $1.1 \times 10^{4}$ & $4.6 \times 10^{-2}$ & $3.6 \times 10^{-2}$ & $4.3 \times 10^{-2}$ \\
\hline 1996 & 325 & 310 & 287 & $9.5 \times 10^{2}$ & $8.1 \times 10^{3}$ & $9.0 \times 10^{3}$ & $4.2 \times 10^{-2}$ & $4.2 \times 10^{-2}$ & $4.5 \times 10^{-2}$ \\
\hline Total & & & & $1.1 \times 10^{6}$ & $5.2 \times 10^{5}$ & $1.6 \times 10^{6}$ & $7.9 \times 10^{0}$ & $3.2 \times 10^{0}$ & $6.1 \times 10^{0}$ \\
\hline
\end{tabular}


Assessment of Radionuclides

Table 4-25. Liquid H-3 Releases and Population Dose

\begin{tabular}{|c|c|c|c|c|c|c|c|c|c|c|}
\hline \multirow[b]{2}{*}{ Year } & \multirow[b]{2}{*}{$\begin{array}{l}\text { River } \\
\text { Flow } \\
\text { Rate } \\
\left(\mathrm{m}^{3} / \mathrm{s}\right)\end{array}$} & \multirow[b]{2}{*}{$\begin{array}{c}\text { BJ } \\
\text { Derived } \\
\text { Flow } \\
\text { Rate } \\
\left(\mathrm{m}^{3} / \mathrm{s}\right)\end{array}$} & \multirow[b]{2}{*}{$\begin{array}{c}\text { PW } \\
\text { Derived } \\
\text { Flow } \\
\text { Rate } \\
\left(\mathrm{m}^{3} / \mathrm{s}\right)\end{array}$} & \multicolumn{3}{|c|}{ Tritium Releases to Streams } & \multicolumn{4}{|c|}{ Population Dose } \\
\hline & & & & $\begin{array}{l}\text { Direct } \\
\text { Release } \\
\text { to } \\
\text { Streams } \\
\text { (Ci) }\end{array}$ & $\begin{array}{l}\text { Migration to } \\
\text { Streams } \\
\text { (Ci) }\end{array}$ & $\begin{array}{c}\text { Total } \\
\text { Release } \\
\text { (Ci) }\end{array}$ & $\begin{array}{c}\text { BJ } \\
\text { Water } \\
\text { Treatment } \\
\text { Plant } \\
\text { (per-rem) }\end{array}$ & $\begin{array}{c}\text { PW } \\
\text { Water } \\
\text { Treatment } \\
\text { Plant } \\
\text { (per-rem) }\end{array}$ & $\begin{array}{c}80 \mathrm{~km} \\
\text { (per-rem) }\end{array}$ & $\begin{array}{c}\text { Total } \\
\text { (per-rem) }\end{array}$ \\
\hline 1954 & 209 & & 263 & & & & & & & \\
\hline 1955 & 169 & & 213 & $5.9 \times 10^{3}$ & $0.0 \times 10^{0}$ & $5.9 \times 10^{3}$ & & $3.0 \times 10^{-1}$ & $1.0 \times 10^{-2}$ & $3.1 \times 10^{-1}$ \\
\hline 1956 & 179 & & 225 & $9.4 \times 10^{3}$ & $0.0 \times 10^{0}$ & $9.4 \times 10^{3}$ & & $4.6 \times 10^{-1}$ & $1.5 \times 10^{-2}$ & $4.8 \times 10^{-1}$ \\
\hline 1957 & 235 & & 297 & $1.5 \times 10^{4}$ & $0.0 \times 10^{0}$ & $1.5 \times 10^{4}$ & & $5.6 \times 10^{-1}$ & $1.9 \times 10^{-2}$ & $5.8 \times 10^{-1}$ \\
\hline 1958 & 313 & & 394 & $2.4 \times 10^{4}$ & $4.0 \times 10^{2}$ & $2.4 \times 10^{4}$ & & $6.8 \times 10^{-1}$ & $2.3 \times 10^{-2}$ & $7.1 \times 10^{-1}$ \\
\hline 1959 & 276 & & 348 & $4.1 \times 10^{4}$ & $8.0 \times 10^{2}$ & $4.2 \times 10^{4}$ & & $1.3 \times 10^{0}$ & $4.5 \times 10^{-2}$ & $1.4 \times 10^{0}$ \\
\hline 1960 & 371 & & 468 & $4.7 \times 10^{4}$ & $1.6 \times 10^{3}$ & $4.9 \times 10^{4}$ & & $1.1 \times 10^{0}$ & $3.8 \times 10^{-2}$ & $1.2 \times 10^{0}$ \\
\hline 1961 & 309 & & 389 & $5.4 \times 10^{4}$ & $2.0 \times 10^{3}$ & $5.6 \times 10^{4}$ & & $1.6 \times 10^{0}$ & $5.3 \times 10^{-2}$ & $1.6 \times 10^{0}$ \\
\hline 1962 & 300 & & 377 & $4.7 \times 10^{4}$ & $1.7 \times 10^{3}$ & $4.8 \times 10^{4}$ & & $1.4 \times 10^{0}$ & $4.7 \times 10^{-2}$ & $1.5 \times 10^{0}$ \\
\hline 1963 & 315 & & 397 & $7.5 \times 10^{4}$ & $2.7 \times 10^{3}$ & $7.7 \times 10^{4}$ & & $2.2 \times 10^{0}$ & $7.2 \times 10^{-2}$ & $2.2 \times 10^{0}$ \\
\hline 1964 & 580 & & 731 & $8.1 \times 10^{4}$ & $4.7 \times 10^{3}$ & $8.5 \times 10^{4}$ & & $1.3 \times 10^{0}$ & $4.3 \times 10^{-2}$ & $1.3 \times 10^{0}$ \\
\hline 1965 & 362 & 800 & 456 & $9.8 \times 10^{4}$ & $5.6 \times 10^{3}$ & $1.0 \times 10^{5}$ & $4.8 \times 10^{0}$ & $2.5 \times 10^{0}$ & $8.5 \times 10^{-2}$ & $7.4 \times 10^{0}$ \\
\hline 1966 & 316 & 520 & 399 & $7.7 \times 10^{4}$ & $4.6 \times 10^{3}$ & $8.2 \times 10^{4}$ & $5.8 \times 10^{0}$ & $2.3 \times 10^{0}$ & $7.6 \times 10^{-2}$ & $8.1 \times 10^{0}$ \\
\hline 1967 & 299 & 625 & 377 & $6.4 \times 10^{4}$ & $5.6 \times 10^{3}$ & $7.0 \times 10^{4}$ & $4.1 \times 10^{0}$ & $2.0 \times 10^{0}$ & $6.9 \times 10^{-2}$ & $6.2 \times 10^{0}$ \\
\hline 1968 & 273 & 470 & 343 & $5.6 \times 10^{4}$ & $5.8 \times 10^{3}$ & $6.2 \times 10^{4}$ & $4.8 \times 10^{0}$ & $2.0 \times 10^{0}$ & $6.6 \times 10^{-2}$ & $6.9 \times 10^{0}$ \\
\hline 1969 & 310 & 624 & 390 & $4.5 \times 10^{4}$ & $1.9 \times 10^{4}$ & $6.4 \times 10^{4}$ & $3.8 \times 10^{0}$ & $1.8 \times 10^{0}$ & $6.1 \times 10^{-2}$ & $5.7 \times 10^{0}$ \\
\hline 1970 & 232 & 772 & 293 & $2.4 \times 10^{4}$ & $1.3 \times 10^{4}$ & $3.7 \times 10^{4}$ & $1.8 \times 10^{0}$ & $1.4 \times 10^{0}$ & $4.7 \times 10^{-2}$ & $3.2 \times 10^{0}$ \\
\hline 1971 & 303 & 1245 & 381 & $2.0 \times 10^{4}$ & $1.8 \times 10^{4}$ & $3.8 \times 10^{4}$ & $1.1 \times 10^{0}$ & $1.1 \times 10^{0}$ & $3.7 \times 10^{-2}$ & $2.3 \times 10^{0}$ \\
\hline 1972 & 318 & 775 & 401 & $2.9 \times 10^{4}$ & $1.7 \times 10^{4}$ & $4.6 \times 10^{4}$ & $2.2 \times 10^{0}$ & $1.3 \times 10^{0}$ & $4.3 \times 10^{-2}$ & $3.5 \times 10^{0}$ \\
\hline 1973 & 409 & 656 & 468 & $4.3 \times 10^{4}$ & $2.8 \times 10^{4}$ & $7.1 \times 10^{4}$ & $4.0 \times 10^{0}$ & $1.7 \times 10^{0}$ & $5.1 \times 10^{-2}$ & $5.7 \times 10^{0}$ \\
\hline 1974 & 314 & 640 & 373 & $3.3 \times 10^{4}$ & $2.7 \times 10^{4}$ & $6.0 \times 10^{4}$ & $3.4 \times 10^{0}$ & $1.8 \times 10^{0}$ & $5.6 \times 10^{-2}$ & $5.3 \times 10^{0}$ \\
\hline 1975 & 436 & 877 & 538 & $2.8 \times 10^{4}$ & $2.8 \times 10^{4}$ & $5.6 \times 10^{4}$ & $2.3 \times 10^{0}$ & $1.1 \times 10^{0}$ & $3.8 \times 10^{-2}$ & $3.5 \times 10^{0}$ \\
\hline 1976 & 394 & 996 & 472 & $3.2 \times 10^{4}$ & $2.8 \times 10^{4}$ & $6.0 \times 10^{4}$ & $2.2 \times 10^{0}$ & $1.4 \times 10^{0}$ & $4.5 \times 10^{-2}$ & $3.6 \times 10^{0}$ \\
\hline 1977 & 330 & 562 & 433 & $2.7 \times 10^{4}$ & $1.7 \times 10^{4}$ & $4.4 \times 10^{4}$ & $2.9 \times 10^{0}$ & $1.1 \times 10^{0}$ & $3.9 \times 10^{-2}$ & $4.0 \times 10^{0}$ \\
\hline 1978 & 298 & 689 & 324 & $1.7 \times 10^{4}$ & $2.0 \times 10^{4}$ & $3.8 \times 10^{4}$ & $2.0 \times 10^{0}$ & $1.3 \times 10^{0}$ & $3.7 \times 10^{-2}$ & $3.3 \times 10^{0}$ \\
\hline 1979 & 375 & 989 & 495 & $1.0 \times 10^{4}$ & $1.9 \times 10^{4}$ & $2.9 \times 10^{4}$ & $1.1 \times 10^{0}$ & $6.6 \times 10^{-1}$ & $2.3 \times 10^{-2}$ & $1.8 \times 10^{0}$ \\
\hline 1980 & 374 & 863 & 488 & $1.0 \times 10^{4}$ & $1.4 \times 10^{4}$ & $2.5 \times 10^{4}$ & $1.1 \times 10^{0}$ & $5.6 \times 10^{-1}$ & $1.9 \times 10^{-2}$ & $1.6 \times 10^{0}$ \\
\hline 1981 & 187 & 331 & 243 & $9.6 \times 10^{3}$ & $1.4 \times 10^{4}$ & $2.4 \times 10^{4}$ & $2.6 \times 10^{0}$ & $1.1 \times 10^{0}$ & $3.7 \times 10^{-2}$ & $3.8 \times 10^{0}$ \\
\hline 1982 & 203 & 555 & 287 & $1.1 \times 10^{4}$ & $2.2 \times 10^{4}$ & $3.3 \times 10^{4}$ & $2.2 \times 10^{0}$ & $1.2 \times 10^{0}$ & $4.7 \times 10^{-2}$ & $3.5 \times 10^{0}$ \\
\hline 1983 & 350 & 361 & 417 & $1.4 \times 10^{4}$ & $2.0 \times 10^{4}$ & $3.4 \times 10^{4}$ & $3.5 \times 10^{0}$ & $9.0 \times 10^{-1}$ & $2.9 \times 10^{-2}$ & $4.4 \times 10^{0}$ \\
\hline 1984 & 361 & 747 & 487 & $1.1 \times 10^{4}$ & $2.2 \times 10^{4}$ & $3.3 \times 10^{4}$ & $1.6 \times 10^{0}$ & $7.4 \times 10^{-1}$ & $2.7 \times 10^{-2}$ & $2.4 \times 10^{0}$ \\
\hline 1985 & 203 & 263 & 254 & $6.1 \times 10^{3}$ & $1.9 \times 10^{4}$ & $2.5 \times 10^{4}$ & $3.5 \times 10^{0}$ & $1.1 \times 10^{0}$ & $3.6 \times 10^{-2}$ & $4.6 \times 10^{0}$ \\
\hline 1986 & 175 & 235 & 213 & $7.3 \times 10^{3}$ & $2.1 \times 10^{4}$ & $2.8 \times 10^{4}$ & $4.4 \times 10^{0}$ & $1.4 \times 10^{0}$ & $4.7 \times 10^{-2}$ & $5.8 \times 10^{0}$ \\
\hline 1987 & 254 & 374 & 357 & $4.5 \times 10^{3}$ & $1.8 \times 10^{4}$ & $2.3 \times 10^{4}$ & $2.2 \times 10^{0}$ & $7.0 \times 10^{-1}$ & $2.6 \times 10^{-2}$ & $3.0 \times 10^{6}$ \\
\hline 1988 & 152 & 211 & 203 & $5.4 \times 10^{3}$ & $1.4 \times 10^{4}$ & $1.9 \times 10^{4}$ & $3.4 \times 10^{0}$ & $1.0 \times 10^{0}$ & $3.7 \times 10^{-2}$ & $4.5 \times 10^{0}$ \\
\hline 1989 & 226 & 240 & 245 & $3.6 \times 10^{3}$ & $1.4 \times 10^{4}$ & $1.7 \times 10^{4}$ & $2.7 \times 10^{0}$ & $7.8 \times 10^{-1}$ & $2.3 \times 10^{-2}$ & $3.5 \times 10^{0}$ \\
\hline 1990 & 336 & 447 & 367 & $2.6 \times 10^{3}$ & $1.4 \times 10^{4}$ & $1.6 \times 10^{4}$ & $1.3 \times 10^{0}$ & $4.9 \times 10^{-1}$ & $1.4 \times 10^{-2}$ & $1.8 \times 10^{0}$ \\
\hline 1991 & 328 & 550 & 453 & $1.1 \times 10^{4}$ & $1.6 \times 10^{4}$ & $2.7 \times 10^{4}$ & $1.8 \times 10^{0}$ & $6.7 \times 10^{-1}$ & $2.5 \times 10^{-2}$ & $2.5 \times 10^{0}$ \\
\hline 1992 & 331 & 350 & 279 & $2.4 \times 10^{3}$ & $1.1 \times 10^{4}$ & $1.4 \times 10^{4}$ & $1.5 \times 10^{0}$ & $5.5 \times 10^{-1}$ & $1.2 \times 10^{-2}$ & $2.0 \times 10^{0}$ \\
\hline 1993 & 419 & 566 & 416 & $1.7 \times 10^{3}$ & $9.6 \times 10^{3}$ & $1.3 \times 10^{4}$ & $8.5 \times 10^{-1}$ & $3.4 \times 10^{-1}$ & $9.1 \times 10^{-3}$ & $1.2 \times 10^{0}$ \\
\hline 1994 & 347 & 421 & 348 & $1.2 \times 10^{3}$ & $9.8 \times 10^{3}$ & $1.1 \times 10^{4}$ & $9.6 \times 10^{-1}$ & $3.5 \times 10^{-1}$ & $9.3 \times 10^{-3}$ & $1.3 \times 10^{0}$ \\
\hline 1995 & 361 & 438 & 369 & $1.3 \times 10^{3}$ & $9.7 \times 10^{3}$ & $1.1 \times 10^{4}$ & $9.2 \times 10^{-1}$ & $3.3 \times 10^{-1}$ & $9.0 \times 10^{-3}$ & $1.3 \times 10^{0}$ \\
\hline 1996 & 325 & 310 & 287 & $9.5 \times 10^{2}$ & $8.1 \times 10^{3}$ & $9.0 \times 10^{3}$ & $1.1 \times 10^{0}$ & $3.5 \times 10^{-9}$ & $8.2 \times 10^{-3}$ & $1.4 \times 10^{0}$ \\
\hline Total & & & & $1.1 \times 10^{6}$ & $5.2 \times 10^{5}$ & $1.6 \times 10^{6}$ & $8.2 \times 10^{1}$ & $4.7 \times 10^{1}$ & $1.6 \times 10^{0}$ & $1.3 \times 10^{2}$ \\
\hline
\end{tabular}


Chapter 4. Releases and Doses

of Individual Radionuclides

WSRC-TR-98-00162

Table 4-26. Atmospheric Uranium Releases and Dose

\begin{tabular}{|c|c|c|c|}
\hline Year & $\begin{array}{l}\text { Release } \\
\text { (Ci) }\end{array}$ & $\begin{array}{c}\text { Maximum } \\
\text { Individual Dose at } \\
\text { Boundary (mrem) }\end{array}$ & $\begin{array}{l}\text { Population Dose } \\
\text { (per-rem) }\end{array}$ \\
\hline \multicolumn{4}{|l|}{1954} \\
\hline 1955 & $3.1 \times 10^{-1}$ & $1.5 \times 10^{-1}$ & $1.1 \times 10^{7}$ \\
\hline 1956 & $1.2 \times 10^{-1}$ & $5.7 \times 10^{-2}$ & $4.4 \times 10^{0}$ \\
\hline 1957 & $2.2 \times 10^{-3}$ & $1.1 \times 10^{-3}$ & $8.1 \times 10^{-2}$ \\
\hline 1958 & $1.6 \times 10^{-3}$ & $7.7 \times 10^{-4}$ & $5.9 \times 10^{-2}$ \\
\hline 1959 & $7.2 \times 10^{-3}$ & $3.5 \times 10^{-3}$ & $2.7 \times 10^{-1}$ \\
\hline 1960 & $2.0 \times 10^{-2}$ & $9.6 \times 10^{-3}$ & $7.4 \times 10^{-1}$ \\
\hline 1961 & $1.0 \times 10^{-2}$ & $4.8 \times 10^{-3}$ & $3.7 \times 10^{-1}$ \\
\hline 1962 & $8.9 \times 10^{-3}$ & $4.3 \times 10^{-3}$ & $3.3 \times 10^{-1}$ \\
\hline 1963 & $3.2 \times 10^{-2}$ & $1.5 \times 10^{-2}$ & $1.2 \times 10^{0}$ \\
\hline 1964 & $5.6 \times 10^{-2}$ & $2.7 \times 10^{-2}$ & $2.1 \times 10^{0}$ \\
\hline 1965 & $5.2 \times 10^{-2}$ & $2.5 \times 10^{-2}$ & $1.9 \times 10^{0}$ \\
\hline 1966 & $2.1 \times 10^{-2}$ & $1.0 \times 10^{-2}$ & $7.8 \times 10^{-1}$ \\
\hline 1967 & $1.9 \times 10^{-2}$ & $9.1 \times 10^{-3}$ & $7.0 \times 10^{-1}$ \\
\hline 1968 & $2.7 \times 10^{-2}$ & $1.3 \times 10^{-2}$ & $1.0 \times 10^{0}$ \\
\hline 1969 & $6.9 \times 10^{-2}$ & $3.3 \times 10^{-2}$ & $2.6 \times 10^{0}$ \\
\hline 1970 & $1.7 \times 10^{-2}$ & $8.2 \times 10^{-3}$ & $6.3 \times 10^{-1}$ \\
\hline 1971 & $5.2 \times 10^{-3}$ & $2.5 \times 10^{-3}$ & $1.9 \times 10^{-1}$ \\
\hline 1972 & $7.7 \times 10^{-3}$ & $3.7 \times 10^{-3}$ & $2.8 \times 10^{-1}$ \\
\hline 1973 & $5.1 \times 10^{-3}$ & $2.4 \times 10^{-3}$ & $1.9 \times 10^{-1}$ \\
\hline 1974 & $8.7 \times 10^{-3}$ & $4.2 \times 10^{-3}$ & $3.2 \times 10^{-1}$ \\
\hline 1975 & $4.7 \times 10^{-3}$ & $2.3 \times 10^{-3}$ & $1.7 \times 10^{-1}$ \\
\hline 1976 & $5.0 \times 10^{-3}$ & $2.4 \times 10^{-3}$ & $1.9 \times 10^{-1}$ \\
\hline 1977 & $1.6 \times 10^{-3}$ & $7.7 \times 10^{-4}$ & $5.9 \times 10^{-2}$ \\
\hline 1978 & $3.1 \times 10^{-3}$ & $1.5 \times 10^{-3}$ & $1.1 \times 10^{-1}$ \\
\hline 1979 & $2.4 \times 10^{-3}$ & $1.2 \times 10^{-3}$ & $8.9 \times 10^{-2}$ \\
\hline 1980 & $4.0 \times 10^{-3}$ & $1.9 \times 10^{-3}$ & $1.5 \times 10^{-1}$ \\
\hline 1981 & $6.1 \times 10^{-3}$ & $2.9 \times 10^{-3}$ & $2.3 \times 10^{-1}$ \\
\hline 1982 & $9.2 \times 10^{-3}$ & $4.4 \times 10^{-3}$ & $3.4 \times 10^{-1}$ \\
\hline 1983 & $4.5 \times 10^{-3}$ & $2.2 \times 10^{-3}$ & $1.7 \times 10^{-1}$ \\
\hline 1984 & $2.2 \times 10^{-3}$ & $1.1 \times 10^{-3}$ & $8.1 \times 10^{-2}$ \\
\hline 1985 & $2.5 \times 10^{-3}$ & $1.2 \times 10^{-3}$ & $9.3 \times 10^{-2}$ \\
\hline 1986 & $1.6 \times 10^{-3}$ & $7.7 \times 10^{-4}$ & $5.9 \times 10^{-2}$ \\
\hline 1987 & $8.5 \times 10^{-3}$ & $4.1 \times 10^{-3}$ & $3.1 \times 10^{-1}$ \\
\hline 1988 & $1.5 \times 10^{-3}$ & $7.2 \times 10^{-4}$ & $5.6 \times 10^{-2}$ \\
\hline 1989 & $5.0 \times 10^{-3}$ & $2.4 \times 10^{-3}$ & $1.9 \times 10^{-1}$ \\
\hline 1990 & $5.2 \times 10^{-3}$ & $2.5 \times 10^{-3}$ & $1.9 \times 10^{-1}$ \\
\hline 1991 & $2.7 \times 10^{-3}$ & $1.3 \times 10^{-3}$ & $1.0 \times 10^{-1}$ \\
\hline 1992 & $1.6 \times 10^{-3}$ & $7.6 \times 10^{-4}$ & $5.8 \times 10^{-2}$ \\
\hline 1993 & $1.9 \times 10^{-3}$ & $9.2 \times 10^{-4}$ & $7.1 \times 10^{-2}$ \\
\hline 1994 & $2.2 \times 10^{-3}$ & $1.1 \times 10^{-3}$ & $8.3 \times 10^{-2}$ \\
\hline 1995 & $1.6 \times 10^{-3}$ & $7.5 \times 10^{-4}$ & $5.8 \times 10^{-2}$ \\
\hline 1996 & $1.7 \times 10^{-3}$ & $8.2 \times 10^{-4}$ & $6.3 \times 10^{-2}$ \\
\hline Total & $8.7 \times 10^{-1}$ & $4.2 \times 10^{-1}$ & $3.2 \times 10^{7}$ \\
\hline
\end{tabular}


Table 4-27. Liquid Uranium Releases and Dose

\begin{tabular}{|c|c|c|c|c|c|c|c|c|c|c|c|}
\hline Year & $\begin{array}{c}\text { River } \\
\text { Flow } \\
\text { Rate } \\
\left(\mathrm{m}^{3} / \mathrm{s}\right)\end{array}$ & \begin{tabular}{|c|} 
BJ \\
Derived \\
Flow \\
Rate \\
$\left(\mathrm{m}^{3} / \mathrm{s}\right)$
\end{tabular} & \begin{tabular}{|c|} 
PW \\
Derived \\
Flow \\
Rate \\
$\left(\mathrm{m}^{3} / \mathrm{s}\right)$
\end{tabular} & $\begin{array}{c}\text { Releases } \\
\text { to } \\
\text { Streams } \\
\text { (Ci) }\end{array}$ & $\begin{array}{c}\text { Max } \\
\text { Ind } \\
\text { Below } \\
\text { SRS } \\
\text { (mrem) }\end{array}$ & $\begin{array}{c}\text { Max } \\
\text { Ind } \\
\text { BJ } \\
\text { (mrem) }\end{array}$ & $\begin{array}{c}\text { Max } \\
\text { Ind } \\
\text { PW } \\
\text { (mrem) }\end{array}$ & $\begin{array}{c}\text { Pop } \\
\text { Dose } \\
\text { BJ } \\
\text { (per-rem) }\end{array}$ & $\begin{array}{c}\text { Pop } \\
\text { Dose } \\
\text { PW } \\
\text { (per-rem) }\end{array}$ & $\begin{array}{c}\text { Pop } \\
\text { Dose } \\
80 \mathrm{~km} \\
\text { (per-rem) }\end{array}$ & $\begin{array}{c}\text { Pop } \\
\text { Dose } \\
\text { Total } \\
\text { (per-rem) }\end{array}$ \\
\hline 1954 & 209 & & 263 & $2.4 \times 10^{-3}$ & $6.5 \times 10^{-5}$ & & $4.9 \times 10^{-5}$ & & $3.6 \times 10^{-4}$ & $1.0 \times 10^{-4}$ & $4.7 \times 10^{-4}$ \\
\hline 1955 & 169 & & 213 & $7.0 \times 10^{-2}$ & $2.3 \times 10^{-3}$ & & $1.8 \times 10^{-3}$ & & $1.3 \times 10^{-2}$ & $3.8 \times 10^{-3}$ & $1.7 \times 10^{-2}$ \\
\hline 1956 & 179 & & 225 & $5.2 \times 10^{-1}$ & $1.6 \times 10^{-2}$ & & $1.2 \times 10^{-2}$ & & $9.2 \times 10^{-2}$ & $2.6 \times 10^{-2}$ & $1.2 \times 10^{-1}$ \\
\hline 1957 & 235 & & 297 & $1.0 \times 10^{-1}$ & $2.4 \times 10^{-3}$ & & $1.8 \times 10^{-3}$ & & $1.3 \times 10^{-2}$ & $3.9 \times 10^{-3}$ & $1.7 \times 10^{-2}$ \\
\hline 1958 & 313 & & 394 & $3.6 \times 10^{-2}$ & $6.5 \times 10^{-4}$ & & $4.9 \times 10^{-4}$ & & $3.6 \times 10^{-3}$ & $1.0 \times 10^{-3}$ & $4.7 \times 10^{-3}$ \\
\hline 1959 & 276 & & 348 & $3.9 \times 10^{-2}$ & $8.0 \times 10^{-4}$ & & $6.0 \times 10^{-4}$ & & $4.4 \times 10^{-3}$ & $1.3 \times 10^{-3}$ & $5.7 \times 10^{-3}$ \\
\hline 1960 & 371 & & 468 & $1.1 \times 10^{-1}$ & $1.7 \times 10^{-3}$ & & $1.3 \times 10^{-3}$ & & $9.3 \times 10^{-3}$ & $2.7 \times 10^{-3}$ & $1.2 \times 10^{-2}$ \\
\hline 1961 & 309 & & 389 & $1.1 \times 10^{-1}$ & $2.0 \times 10^{-3}$ & & $1.5 \times 10^{-3}$ & & $1.1 \times 10^{-2}$ & $3.2 \times 10^{-3}$ & $1.4 \times 10^{-2}$ \\
\hline 1962 & 300 & & 377 & $5.3 \times 10^{-1}$ & $1.0 \times 10^{-2}$ & & $7.6 \times 10^{-3}$ & & $5.6 \times 10^{-2}$ & $1.6 \times 10^{-2}$ & $7.2 \times 10^{-2}$ \\
\hline 1963 & 315 & & 397 & $2.0 \times 10^{0}$ & $3.6 \times 10^{-2}$ & & $2.7 \times 10^{-2}$ & & $2.0 \times 10^{-1}$ & $5.7 \times 10^{-2}$ & $2.6 \times 10^{-1}$ \\
\hline 1964 & 580 & & 731 & $5.0 \times 10^{-1}$ & $4.9 \times 10^{-3}$ & & $3.7 \times 10^{-3}$ & & $2.7 \times 10^{-2}$ & $7.8 \times 10^{-3}$ & $3.5 \times 10^{-2}$ \\
\hline 1965 & 362 & 800 & 456 & $9.0 \times 10^{-1}$ & $1.4 \times 10^{-2}$ & $6.0 \times 10^{-3}$ & $1.1 \times 10^{-2}$ & $1.5 \times 10^{-1}$ & $7.8 \times 10^{-2}$ & $2.3 \times 10^{-2}$ & $2.5 \times 10^{-1}$ \\
\hline 1966 & 316 & 520 & 399 & $4.4 \times 10^{\circ}$ & $7.9 \times 10^{-2}$ & $4.5 \times 10^{-2}$ & $5.9 \times 10^{-2}$ & $1.1 \times 10^{0}$ & $4.4 \times 10^{-1}$ & $1.3 \times 10^{-1}$ & $1.7 \times 10^{0}$ \\
\hline 1967 & 299 & 625 & 377 & $6.4 \times 10^{0}$ & $1.2 \times 10^{-1}$ & $5.5 \times 10^{-2}$ & $9.1 \times 10^{-2}$ & $1.4 \times 10^{0}$ & $6.7 \times 10^{-1}$ & $1.9 \times 10^{-1}$ & $2.3 \times 10^{0}$ \\
\hline 1968 & 273 & 470 & 343 & $4.2 \times 10^{0}$ & $8.7 \times 10^{-2}$ & $4.8 \times 10^{-2}$ & $6.6 \times 10^{-2}$ & $1.2 \times 10^{0}$ & $4.9 \times 10^{-1}$ & $1.4 \times 10^{-1}$ & $1.8 \times 10^{0}$ \\
\hline 1969 & 310 & 624 & 390 & $1.3 \times 10^{0}$ & $2.4 \times 10^{-2}$ & $1.1 \times 10^{-2}$ & $1.8 \times 10^{-2}$ & $2.8 \times 10^{-1}$ & $1.3 \times 10^{-1}$ & $3.8 \times 10^{-2}$ & $4.5 \times 10^{-1}$ \\
\hline 1970 & 232 & 772 & 293 & $6.8 \times 10^{-1}$ & $1.7 \times 10^{-2}$ & $4.7 \times 10^{-3}$ & $1.2 \times 10^{-2}$ & $1.2 \times 10^{-1}$ & $9.2 \times 10^{-2}$ & $2.7 \times 10^{-2}$ & $2.4 \times 10^{-1}$ \\
\hline 1971 & 303 & 1245 & 381 & $3.5 \times 10^{-1}$ & $6.5 \times 10^{-3}$ & $1.5 \times 10^{-3}$ & $4.9 \times 10^{-3}$ & $3.8 \times 10^{-2}$ & $3.6 \times 10^{-2}$ & $1.0 \times 10^{-2}$ & $8.5 \times 10^{-2}$ \\
\hline 1972 & 318 & 775 & 401 & $3.9 \times 10^{-1}$ & $6.9 \times 10^{-3}$ & $2.7 \times 10^{-3}$ & $5.2 \times 10^{-3}$ & $6.8 \times 10^{-2}$ & $3.9 \times 10^{-2}$ & $1.1 \times 10^{-2}$ & $1.2 \times 10^{-1}$ \\
\hline 1973 & 409 & 656 & 468 & $2.2 \times 10^{-1}$ & $3.0 \times 10^{-3}$ & $1.8 \times 10^{-3}$ & $2.5 \times 10^{-3}$ & $4.6 \times 10^{-2}$ & $1.9 \times 10^{-2}$ & $4.9 \times 10^{-3}$ & $6.9 \times 10^{-2}$ \\
\hline 1974 & 314 & 640 & 373 & $3.5 \times 10^{-1}$ & $6.3 \times 10^{-3}$ & $2.9 \times 10^{-3}$ & $5.0 \times 10^{-3}$ & $7.4 \times 10^{-2}$ & $3.7 \times 10^{-2}$ & $1.0 \times 10^{-2}$ & $1.2 \times 10^{-1}$ \\
\hline 1975 & 436 & 877 & 538 & $4.4 \times 10^{-1}$ & $5.7 \times 10^{-3}$ & $2.7 \times 10^{-3}$ & $4.4 \times 10^{-3}$ & $6.8 \times 10^{-2}$ & $3.2 \times 10^{-2}$ & $9.1 \times 10^{-3}$ & $1.1 \times 10^{-1}$ \\
\hline 1976 & 394 & 996 & 472 & $3.6 \times 10^{-1}$ & $5.2 \times 10^{-3}$ & $1.9 \times 10^{-3}$ & $4.1 \times 10^{-3}$ & $4.9 \times 10^{-2}$ & $3.0 \times 10^{-2}$ & $8.3 \times 10^{-3}$ & $8.8 \times 10^{-2}$ \\
\hline 1977 & 330 & 562 & 433 & $8.4 \times 10^{-2}$ & $1.4 \times 10^{-3}$ & $8.0 \times 10^{-4}$ & $1.0 \times 10^{-3}$ & $2.0 \times 10^{-2}$ & $7.7 \times 10^{-3}$ & $2.3 \times 10^{-3}$ & $3.0 \times 10^{-2}$ \\
\hline 1978 & 298 & 689 & 324 & $5.6 \times 10^{-2}$ & $1.1 \times 10^{-3}$ & $4.4 \times 10^{-4}$ & $9.3 \times 10^{-4}$ & $1.1 \times 10^{-2}$ & $6.8 \times 10^{-3}$ & $1.7 \times 10^{-3}$ & $2.0 \times 10^{-2}$ \\
\hline 1979 & 375 & 989 & 495 & $6.4 \times 10^{-2}$ & $9.7 \times 10^{-4}$ & $3.5 \times 10^{-4}$ & $7.0 \times 10^{-4}$ & $8.8 \times 10^{-3}$ & $5.1 \times 10^{-3}$ & $1.5 \times 10^{-3}$ & $1.5 \times 10^{-2}$ \\
\hline 1980 & 374 & 863 & 488 & $1.9 \times 10^{-1}$ & $2.9 \times 10^{-3}$ & $1.2 \times 10^{-3}$ & $2.1 \times 10^{-3}$ & $3.0 \times 10^{-2}$ & $1.5 \times 10^{-2}$ & $4.6 \times 10^{-3}$ & $5.0 \times 10^{-2}$ \\
\hline 1981 & 187 & 331 & 243 & $1.7 \times 10^{-1}$ & $5.1 \times 10^{-3}$ & $2.8 \times 10^{-3}$ & $3.8 \times 10^{-3}$ & $7.0 \times 10^{-2}$ & $2.8 \times 10^{-2}$ & $8.2 \times 10^{-3}$ & $1.1 \times 10^{-1}$ \\
\hline 1982 & 203 & 555 & 287 & $4.2 \times 10^{-2}$ & $1.2 \times 10^{-3}$ & $4.1 \times 10^{-4}$ & $7.9 \times 10^{-4}$ & $1.0 \times 10^{-2}$ & $5.8 \times 10^{-3}$ & $1.9 \times 10^{-3}$ & $1.8 \times 10^{-2}$ \\
\hline 1983 & 350 & 361 & 417 & $1.7 \times 10^{-2}$ & $2.7 \times 10^{-4}$ & $2.5 \times 10^{-4}$ & $2.2 \times 10^{-4}$ & $6.4 \times 10^{-3}$ & $1.6 \times 10^{-3}$ & $4.4 \times 10^{-4}$ & $8.5 \times 10^{-3}$ \\
\hline 1984 & 361 & 747 & 487 & $9.7 \times 10^{-3}$ & $1.5 \times 10^{-4}$ & $7.0 \times 10^{-5}$ & $1.1 \times 10^{-4}$ & $1.8 \times 10^{-3}$ & $7.9 \times 10^{-4}$ & $2.4 \times 10^{-4}$ & $2.8 \times 10^{-3}$ \\
\hline 1985 & 203 & 263 & 254 & $1.4 \times 10^{-3}$ & $3.9 \times 10^{-5}$ & $2.9 \times 10^{-5}$ & $3.0 \times 10^{-5}$ & $7.2 \times 10^{-4}$ & $2.2 \times 10^{-4}$ & $6.2 \times 10^{-5}$ & $1.0 \times 10^{-3}$ \\
\hline 1986 & 175 & 235 & 213 & $4.4 \times 10^{-2}$ & $1.4 \times 10^{-3}$ & $1.0 \times 10^{-3}$ & $1.1 \times 10^{-3}$ & $2.5 \times 10^{-2}$ & $8.2 \times 10^{-3}$ & $2.3 \times 10^{-3}$ & $3.6 \times 10^{-2}$ \\
\hline 1987 & 254 & 374 & 357 & $5.5 \times 10^{-3}$ & $1.2 \times 10^{-4}$ & $7.9 \times 10^{-5}$ & $8.3 \times 10^{-5}$ & $2.0 \times 10^{-3}$ & $6.1 \times 10^{-4}$ & $2.0 \times 10^{-4}$ & $2.8 \times 10^{-3}$ \\
\hline 1988 & 152 & 211 & 203 & $5.5 \times 10^{-3}$ & $2.0 \times 10^{-4}$ & $1.4 \times 10^{-4}$ & $1.5 \times 10^{-4}$ & $3.5 \times 10^{-3}$ & $1.1 \times 10^{-3}$ & $3.3 \times 10^{-4}$ & $4.9 \times 10^{-3}$ \\
\hline 1989 & 226 & 240 & 245 & $4.2 \times 10^{-4}$ & $1.1 \times 10^{-5}$ & $9.4 \times 10^{-6}$ & $9.2 \times 10^{-6}$ & $2.4 \times 10^{-4}$ & $6.8 \times 10^{-5}$ & $1.7 \times 10^{-5}$ & $3.2 \times 10^{-4}$ \\
\hline 1990 & 336 & 447 & 367 & $1.4 \times 10^{-3}$ & $2.4 \times 10^{-5}$ & $1.7 \times 10^{-5}$ & $2.1 \times 10^{-5}$ & $4.3 \times 10^{-4}$ & $1.5 \times 10^{-4}$ & $3.8 \times 10^{-5}$ & $6.1 \times 10^{-4}$ \\
\hline 1991 & 328 & 550 & 453 & $3.2 \times 10^{-3}$ & $5.5 \times 10^{-5}$ & $3.1 \times 10^{-5}$ & $3.8 \times 10^{-5}$ & $7.9 \times 10^{-4}$ & $2.8 \times 10^{-4}$ & $8.8 \times 10^{-5}$ & $1.2 \times 10^{-3}$ \\
\hline 1992 & 331 & 350 & 279 & $1.9 \times 10^{-3}$ & $3.2 \times 10^{-5}$ & $2.9 \times 10^{-5}$ & $3.7 \times 10^{-5}$ & $7.4 \times 10^{-4}$ & $2.7 \times 10^{-4}$ & $5.2 \times 10^{-5}$ & $1.1 \times 10^{-3}$ \\
\hline 1993 & 419 & 566 & 416 & $1.1 \times 10^{-5}$ & $1.5 \times 10^{-7}$ & $1.0 \times 10^{-7}$ & $1.4 \times 10^{-7}$ & $2.6 \times 10^{-6}$ & $1.0 \times 10^{-6}$ & $2.4 \times 10^{-7}$ & $3.9 \times 10^{-6}$ \\
\hline 1994 & 347 & 421 & 348 & $1.0 \times 10^{-5}$ & $1.6 \times 10^{-7}$ & $1.3 \times 10^{-7}$ & $1.5 \times 10^{-7}$ & $3.2 \times 10^{-6}$ & $1.1 \times 10^{-6}$ & $2.6 \times 10^{-7}$ & $4.6 \times 10^{-6}$ \\
\hline 1995 & 361 & 438 & 369 & $3.3 \times 10^{-4}$ & $5.2 \times 10^{-6}$ & $4.1 \times 10^{-6}$ & $4.8 \times 10^{-6}$ & $1.0 \times 10^{-4}$ & $3.5 \times 10^{-5}$ & $8.3 \times 10^{-6}$ & $1.5 \times 10^{-4}$ \\
\hline 1996 & 325 & 310 & 287 & $1.9 \times 10^{-2}$ & $3.3 \times 10^{-4}$ & $3.3 \times 10^{-4}$ & $3.5 \times 10^{-4}$ & $8.3 \times 10^{-3}$ & $2.6 \times 10^{-3}$ & $5.3 \times 10^{-4}$ & $1.1 \times 10^{-2}$ \\
\hline Total & & & & $2.5 \times 10^{1}$ & $4.7 \times 10^{-1}$ & $1.9 \times 10^{-1}$ & $3.5 \times 10^{-1}$ & $4.9 \times 10^{\circ}$ & $2.6 \times 10^{0}$ & $7.5 \times 10^{-1}$ & $8.2 \times 10^{0}$ \\
\hline
\end{tabular}


-

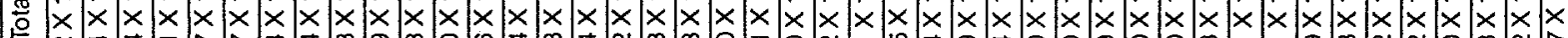

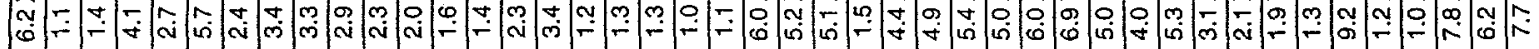

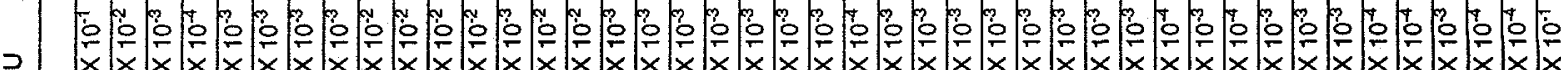

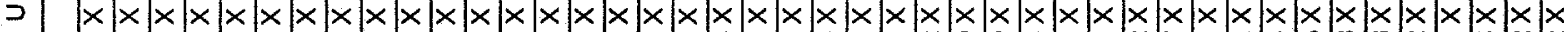

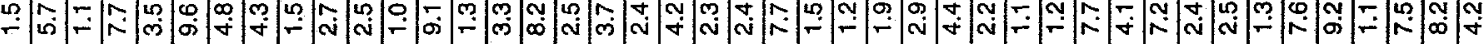

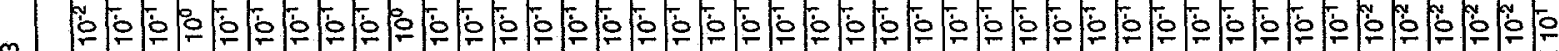

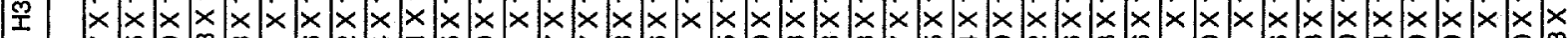

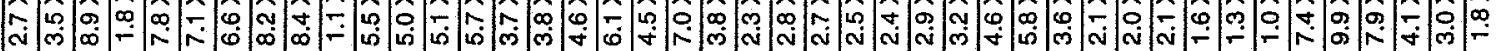

8

$\dot{0}$

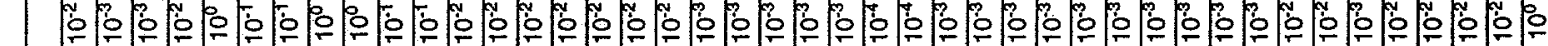

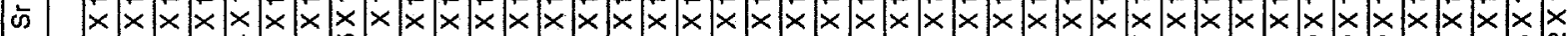

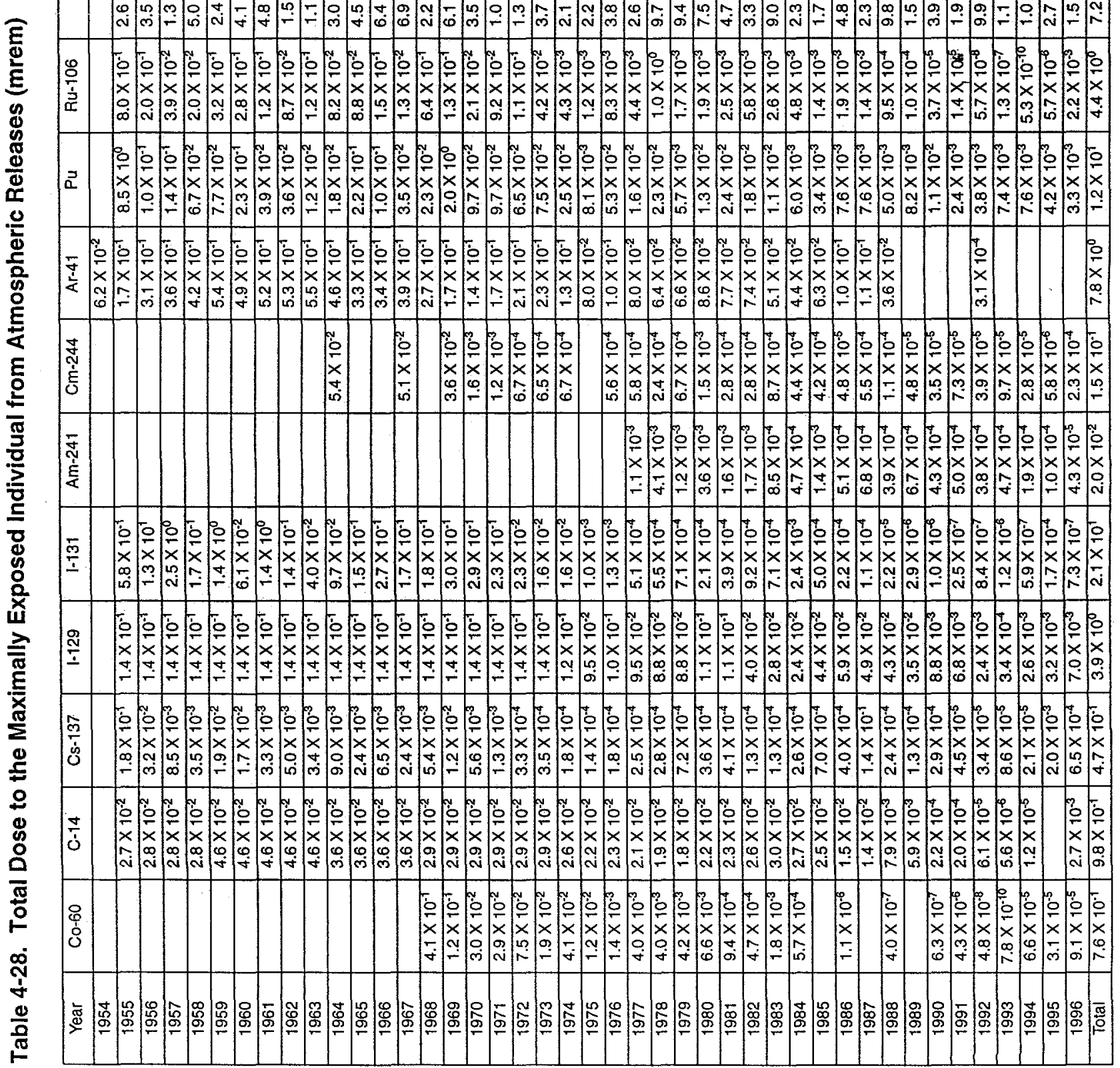


Table 4-29. Population Dose from Atmospheric Releases (person-rem)

\begin{tabular}{|c|c|c|c|c|c|c|c|c|c|c|c|c|c|c|c|}
\hline Year & Co-60 & C-14 & Cs. 137 & $1-129$ & $1-131$ & Am-241 & $\mathrm{Cm}-244$ & $\mathrm{Ar}-41$ & $\mathrm{Pu}$ & Ru-106 & Sr & TC-99 & $\mathrm{H} 3$ & $\bar{U}$ & Total \\
\hline 1954 & & & & & & & & $1.5 \times 10^{0}$ & & & & & & & $1.5 \times 10^{0}$ \\
\hline 1955 & & $8.3 \times 10^{-1}$ & $1.3 \times 10^{1}$ & $3.8 \times 10^{\circ}$ & $2.3 \times 10^{1}$ & & & $4.2 \times 10^{0}$ & $5.0 \times 10^{2}$ & $2.2 \times 10^{1}$ & $7.7 \times 10^{-1}$ & & $1.7 \times 10^{0}$ & $1.1 \times 10^{1}$ & $5.8 \times 10^{2}$ \\
\hline 1956 & & $8.4 \times 10^{-1}$ & $2.3 \times 10^{0}$ & $3.8 \times 10^{0}$ & $5.2 \times 10^{2}$ & & & $7.5 \times 10^{0}$ & $5.8 \times 10^{0}$ & $5.4 \times 10^{\circ}$ & $1.0 \times 10^{-1}$ & & $2.2 \times 10^{1}$ & $4.4 \times 10^{\circ}$ & $5.7 \times 10^{2}$ \\
\hline 1957 & & $8.4 \times 10^{-1}$ & $6.1 \times 10^{-4}$ & $3.8 \times 10^{0}$ & $9.7 \times 10^{1}$ & & & $8.7 \times 10^{0}$ & $8.0 \times 10^{\circ}$ & $1.0 \times 10^{0}$ & $3.8 \times 10^{-2}$ & & $5.6 \times 10^{1}$ & $8.1 \times 10^{-2}$ & $1.8 \times 10^{2}$ \\
\hline 1958 & & $8.4 \times 10^{-1}$ & $2.5 \times 10^{-1}$ & $3.8 \times 10^{0}$ & $6.6 \times 10^{0}$ & & & $1.0 \times 10^{1}$ & $3.9 \times 10^{0}$ & $5.3 \times 10^{-1}$ & $6.7 \times 10^{-2}$ & & $1.1 \times 10^{2}$ & $5.9 \times 10^{-2}$ & $1.4 \times 10^{2}$ \\
\hline 1959 & & $1.4 \times 10^{0}$ & $1.3 \times 10^{0}$ & $3.8 \times 10^{\circ}$ & $5.4 \times 10^{1}$ & & & $1.3 \times 10^{1}$ & $4.5 \times 10^{0}$ & $8.5 \times 10^{0}$ & $1.8 \times 10^{0}$ & & $4.9 \times 10^{1}$ & $2.7 \times 10^{-1}$ & $1.4 \times 10^{2}$ \\
\hline 1960 & & $1.4 \times 10^{0}$ & $1.2 \times 10^{0}$ & $3.8 \times 10^{\circ}$ & $2.4 \times 10^{\circ}$ & & & $1.2 \times 10^{1}$ & $1.3 \times 10^{1}$ & $7.5 \times 10^{0}$ & $3.0 \times 10^{-1}$ & & $4.4 \times 10^{1}$ & $7.4 \times 10^{-1}$ & $8.6 \times 10^{1}$ \\
\hline 1961 & & $1.4 \times 10^{0}$ & $2.4 \times 10^{-1}$ & $3.8 \times 10^{0}$ & $5.4 \times 10^{1}$ & & & $1.3 \times 10^{1}$ & $2.3 \times 10^{0}$ & $3.3 \times 10^{0}$ & $3.1 \times 10^{-1}$ & & $4.1 \times 10^{1}$ & $3.7 \times 10^{-1}$ & $1.2 \times 10^{2}$ \\
\hline 1962 & & $1.4 \times 10^{0}$ & $3.6 \times 10^{-4}$ & $3.8 \times 10^{0}$ & $5.5 \times 10^{0}$ & & & $1.3 \times 10^{1}$ & $2.1 \times 10^{0}$ & $2.3 \times 10^{0}$ & $8.1 \times 10^{-1}$ & & $5.1 \times 10^{1}$ & $3.3 \times 10^{-1}$ & $8.1 \times 10^{1}$ \\
\hline 1963 & & $1.4 \times 10^{0}$ & $2.4 \times 10^{-1}$ & $3.8 \times 10^{0}$ & $1.6 \times 10^{0}$ & & & $1.4 \times 10^{1}$ & $7.0 \times 10^{-1}$ & $3.3 \times 10^{0}$ & $6.0 \times 10^{-1}$ & & $5.2 \times 10^{1}$ & $1.2 \times 10^{0}$ & $7.9 \times 10^{1}$ \\
\hline 1964 & & $1.1 \times 10^{\circ}$ & $6.4 \times 10^{-1}$ & $3.8 \times 10^{0}$ & $3.8 \times 10^{0}$ & & $3.1 \times 10^{0}$ & $1.1 \times 10^{1}$ & $1.0 \times 10^{\circ}$ & $2.2 \times 10^{\circ}$ & $2.2 \times 10^{-1}$ & & $7.0 \times 10^{1}$ & $2.1 \times 10^{0}$ & $9.9 \times 10^{1}$ \\
\hline 1965 &. & $1.1 \times 10^{0}$ & $1.7 \times 10^{-1}$ & $3.8 \times 10^{0}$ & $6.0 \times 10^{\circ}$ & & & $8.1 \times 10^{\circ}$ & $1.3 \times 10^{1}$ & $2.4 \times 10^{0}$ & $2.6 \times 10^{-1}$ & & $3.4 \times 10^{1}$ & $1.9 \times 10^{0}$ & $7.1 \times 10^{1}$ \\
\hline 1966 & & $1.1 \times 10^{0}$ & $4.7 \times 10^{-1}$ & $3.8 \times 10^{0}$ & $1.0 \times 10^{1}$ & & & $8.4 \times 10^{0}$ & $5.8 \times 10^{0}$ & $3.9 \times 10^{0}$ & $6.9 \times 10^{-2}$ & & $3.1 \times 10^{1}$ & $7.8 \times 10^{-1}$ & $6.5 \times 10^{1}$ \\
\hline 1967 & & $1.1 \times 10^{0}$ & $1.7 \times 10^{-1}$ & $3.8 \times 10^{0}$ & $6.6 \times 10^{0}$ & & $3.0 \times 10^{0}$ & $9.6 \times 10^{0}$ & $2.0 \times 10^{0}$ & $3.6 \times 10^{-1}$ & $5.9 \times 10^{-2}$ & & $3.2 \times 10^{\dagger}$ & $7.0 \times 10^{-1}$ & $5.9 \times 10^{1}$ \\
\hline 1968 & $2.8 \times 10^{-1}$ & $8.7 \times 10^{-1}$ & $3.9 \times 10^{-1}$ & $3.8 \times 10^{0}$ & $7.3 \times 10^{\circ}$ & & & $6.6 \times 10^{\circ}$ & $1.3 \times 10^{0}$ & $1.7 \times 10^{1}$ & $7.1 \times 10^{-2}$ & & $3.5 \times 10^{1}$ & $1.0 \times 10^{0}$ & $7.4 \times 10^{1}$ \\
\hline 1969 & $7.8 \times 10^{-2}$ & $8.7 \times 10^{-1}$ & $8.2 \times 10^{-1}$ & $3.8 \times 10^{0}$ & $1.2 \times 10^{1}$ & & $2.1 \times 10^{0}$ & $4.2 \times 10^{0}$ & $1.2 \times 10^{2}$ & $3.5 \times 10^{0}$ & $2.3 \times 10^{-1}$ & & $2.3 \times 10^{1}$ & $2.6 \times 10^{0}$ & $1.7 \times 10^{2}$ \\
\hline 1970 & $2.0 \times 10^{-2}$ & $8.8 \times 10^{-1}$ & $4.0 \times 10^{-1}$ & $3.8 \times 10^{0}$ & $1.1 \times 10^{1}$ & & $9.0 \times 10^{-2}$ & $3.3 \times 10^{0}$ & $5.7 \times 10^{0}$ & $5.8 \times 10^{-1}$ & $1.2 \times 10^{-1}$ & & $2.4 \times 10^{1}$ & $6.3 \times 10^{-1}$ & $5.1 \times 10^{1}$ \\
\hline 1971 & $1.6 \times 10^{-1}$ & $8.7 \times 10^{-1}$ & $9.0 \times 10^{-2}$ & $3.8 \times 10^{0}$ & $8.9 \times 10^{0}$ & & $6.9 \times 10^{-2}$ & $4.2 \times 10^{0}$ & $5.7 \times 10^{0}$ & $2.5 \times 10^{0}$ & $6.9 \times 10^{-2}$ & & $2.9 \times 10^{1}$ & $1.9 \times 10^{-1}$ & $5.6 \times 10^{1}$ \\
\hline 1972 & $5.1 \times 10^{-2}$ & $8.7 \times 10^{-1}$ & $2.4 \times 10^{-2}$ & $3.8 \times 10^{0}$ & $8.9 \times 10^{-1}$ & & $3.9 \times 10^{-2}$ & $5.0 \times 10^{\circ}$ & $3.8 \times 10^{\circ}$ & $2.9 \times 10^{0}$ & $2.4 \times 10^{-1}$ & & $3.8 \times 10^{9}$ & $2.8 \times 10^{-1}$ & $5.6 \times 10^{1}$ \\
\hline 1973 & $1.3 \times 10^{-2}$ & $8.7 \times 10^{-1}$ & $2.5 \times 10^{-2}$ & $3.8 \times 10^{0}$ & $6.3 \times 10^{-1}$ & & $3.8 \times 10^{-2}$ & $5.6 \times 10^{\circ}$ & $4.4 \times 10^{0}$ & $1.1 \times 10^{0}$ & $2.7 \times 10^{-2}$ & & $2.8 \times 10^{1}$ & $1.9 \times 10^{-1}$ & $4.5 \times 10^{1}$ \\
\hline 1974 & $2.8 \times 10^{-2}$ & $8.0 \times 10^{-1}$ & $1.3 \times 10^{-2}$ & $3.1 \times 10^{0}$ & $6.3 \times 10^{-1}$ & & $3.9 \times 10^{-2}$ & $3.2 \times 10^{0}$ & $1.4 \times 10^{0}$ & $1.2 \times 10^{-1}$ & $2.0 \times 10^{-2}$ & & $4.3 \times 10^{1}$ & $3.2 \times 10^{-1}$ & $5.3 \times 10^{1}$ \\
\hline 1975 & $7.8 \times 10^{-3}$ & $6.6 \times 10^{-1}$ & $1.0 \times 10^{-2}$ & $2.5 \times 10^{0}$ & $4.1 \times 10^{-2}$ & & & $2.0 \times 10^{0}$ & $4.7 \times 10^{-1}$ & $3.2 \times 10^{\circ}$ & $9.9 \times 10^{-3}$ & & $2.4 \times 10^{1}$ & $1.7 \times 10^{-1}$ & $3.0 \times 10^{1}$ \\
\hline 1976 & $9.3 \times 10^{-4}$ & $6.9 \times 10^{-1}$ & $1.3 \times 10^{-2}$ & $2.7 \times 10^{0}$ & $5.3 \times 10^{-2}$ & & $3.3 \times 10^{-2}$ & $2.5 \times 10^{0}$ & $3.1 \times 10^{0}$ & $2.2 \times 10^{-1}$ & $1.0 \times 10^{-2}$ & & $1.4 \times 10^{1}$ & $1.9 \times 10^{-1}$ & $2.4 \times 10^{1}$ \\
\hline 1977 & $2.7 \times 10^{-3}$ & $6.3 \times 10^{-1}$ & $1.8 \times 10^{-2}$ & $2.5 \times 10^{0}$ & $2.0 \times 10^{-2}$ & $6.5 \times 10^{-2}$ & $3.4 \times 10^{-2}$ & $2.0 \times 10^{0}$ & $9.6 \times 10^{-1}$ & $1.2 \times 10^{-1}$ & $8.6 \times 10^{3}$ & & $1.8 \times 10^{1}$ & $5.9 \times 10^{-2}$ & $2.4 \times 10^{1}$ \\
\hline 1978 & $2.7 \times 10^{-3}$ & $5.7 \times 10^{-1}$ & $2.0 \times 10^{-2}$ & $2.4 \times 10^{0}$ & $2.2 \times 10^{-2}$ & $2.4 \times 10^{-1}$ & $1.4 \times 10^{-2}$ & $1.6 \times 10^{0}$ & $1.3 \times 10^{0}$ & $2.7 \times 10^{1}$ & $7.2 \times 10^{-3}$ & & $1.7 \times 10^{1}$ & $1.1 \times 10^{-1}$ & $5.0 \times 10^{1}$ \\
\hline 1979 & $2.8 \times 10^{3}$ & $5.6 \times 10^{-1}$ & $5.2 \times 10^{-2}$ & $2.4 \times 10^{0}$ & $2.8 \times 10^{-2}$ & $6.8 \times 10^{-2}$ & $3.9 \times 10^{-2}$ & $1.6 \times 10^{0}$ & $3.3 \times 10^{-1}$ & $4.7 \times 10^{\circ}$ & $4.9 \times 10^{-3}$ & & $1.5 \times 10^{1}$ & $8.9 \times 10^{\circ 2}$ & $2.0 \times 10^{1}$ \\
\hline 1980 & $4.4 \times 10^{-3}$ & $6.6 \times 10^{-1}$ & $2.5 \times 10^{-2}$ & $2.9 \times 10^{0}$ & $8.3 \times 10^{-3}$ & $2.1 \times 10^{-1}$ & $8.9 \times 10^{-2}$ & $2.1 \times 10^{0}$ & $7.4 \times 10^{-1}$ & $5.1 \times 10^{-2}$ & $8.3 \times 10^{-3}$ & & $1.5 \times 10^{1}$ & $1.5 \times 10^{-1}$ & $2.2 \times 10^{1}$ \\
\hline 1981 & $6.3 \times 10^{-4}$ & $6.9 \times 10^{-1}$ & $3.0 \times 10^{-2}$ & $2.9 \times 10^{0}$ & $1.6 \times 10^{-2}$ & $9.5 \times 10^{-2}$ & $1.6 \times 10^{-2}$ & $1.9 \times 10^{\circ}$ & $1.4 \times 10^{\circ}$ & $6.7 \times 10^{-2}$ & $7.6 \times 10^{-3}$ & & $1.8 \times 10^{1}$ & $2.3 \times 10^{-1}$ & $2.5 \times 10^{1}$ \\
\hline 1982 & $3.1 \times 10^{-4}$ & $8.0 \times 10^{-1}$ & $9.3 \times 10^{-3}$ & $1.1 \times 10^{0}$ & $3.6 \times 10^{-2}$ & $9.6 \times 10^{-2}$ & $1.6 \times 10^{-2}$ & $1.8 \times 10^{\circ}$ & $1.1 \times 10^{\circ}$ & $1.6 \times 10^{-1}$ & $5.7 \times 10^{-3}$ & & $2.0 \times 10^{1}$ & $3.4 \times 10^{-1}$ & $2.5 \times 10^{1}$ \\
\hline 1983 & $1.2 \times 10^{-3}$ & $9.0 \times 10^{-1}$ & $9.6 \times 10^{-3}$ & $7.4 \times 10^{-1}$ & $2.8 \times 10^{-2}$ & $4.9 \times 10^{-2}$ & $5.0 \times 10^{-2}$ & $1.2 \times 10^{0}$ & $6.2 \times 10^{-1}$ & $6.9 \times 10^{-2}$ & $9.2 \times 10^{-3}$ & & $2.9 \times 10^{1}$ & $1.7 \times 10^{-1}$ & $3.3 \times 10^{4}$ \\
\hline 1984 & $3.8 \times 10^{-4}$ & $8.3 \times 10^{-1}$ & $1.9 \times 10^{-2}$ & $6.3 \times 10^{-1}$ & $9.3 \times 10^{-2}$ & $2.7 \times 10^{-2}$ & $2.6 \times 10^{-2}$ & $1.1 \times 10^{0}$ & $3.5 \times 10^{-1}$ & $1.3 \times 10^{-1}$ & $7.3 \times 10^{-3}$ & & $3.6 \times 10^{1}$ & $8.1 \times 10^{-2}$ & $3.9 \times 10^{1}$ \\
\hline 1985 & & $7.6 \times 10^{-1}$ & $5.0 \times 10^{-2}$ & $1.2 \times 10^{0}$ & $2.0 \times 10^{-2}$ & $8.2 \times 10^{-2}$ & $2.4 \times 10^{-2}$ & $1.5 \times 10^{0}$ & $2.0 \times 10^{-1}$ & $3.7 \times 10^{-2}$ & $4.0 \times 10^{-3}$ & & $2.3 \times 10^{1}$ & $9.3 \times 10^{-2}$ & $2.7 \times 10^{1}$ \\
\hline 1986 & $5.7 \times 10^{-5}$ & $4.6 \times 10^{-1}$ & $2.8 \times 10^{-2}$ & $1.6 \times 10^{0}$ & $8.6 \times 10^{-3}$ & $3.0 \times 10^{-2}$ & $2.8 \times 10^{-3}$ & $2.5 \times 10^{0}$ & $4.4 \times 10^{-1}$ & $5.0 \times 10^{-2}$ & $4.0 \times 10^{-3}$ & & $1.3 \times 10^{1}$ & $5.9 \times 10^{-2}$ & $1.8 \times 10^{1}$ \\
\hline 1987 & & $4.1 \times 10^{-1}$ & $1.0 \times 10^{1}$ & $1.3 \times 10^{0}$ & $4.3 \times 10^{-3}$ & $3.9 \times 10^{-2}$ & $3.2 \times 10^{-2}$ & $2.6 \times 10^{0}$ & $4.4 \times 10^{-4}$ & $3.8 \times 10^{-2}$ & $2.3 \times 10^{-3}$ & & $1.3 \times 10^{1}$ & $3.1 \times 10^{-1}$ & $2.8 \times 10^{1}$ \\
\hline 1988 & $2.1 \times 10^{-5}$ & $2.4 \times 10^{-1}$ & $1.7 \times 10^{-2}$ & $1.1 \times 10^{0}$ & $8.6 \times 10^{-4}$ & $2.3 \times 10^{-2}$ & $6.6 \times 10^{-3}$ & $8.9 \times 10^{-1}$ & $2.9 \times 10^{-1}$ & $2.6 \times 10^{-2}$ & $6.6 \times 10^{-3}$ & & $1.3 \times 10^{1}$ & $5.6 \times 10^{-2}$ & $1.6 \times 10^{1}$ \\
\hline 1989 & & $1.8 \times 10^{-1}$ & $9.2 \times 10^{-3}$ & $9.4 \times 10^{-1}$ & $1.1 \times 10^{-4}$ & $3.9 \times 10^{-2}$ & $2.8 \times 10^{-3}$ & & $4.8 \times 10^{-1}$ & $2.8 \times 10^{-3}$ & $1.7 \times 10^{-3}$ & & $1.0 \times 10^{9}$ & $1.9 \times 10^{-1}$ & $1.2 \times 10^{9}$ \\
\hline 1990 & $3.4 \times 10^{-5}$ & $6.7 \times 10^{-3}$ & $2.1 \times 10^{-2}$ & $2.4 \times 10^{-1}$ & $4.1 \times 10^{-5}$ & $2.5 \times 10^{-2}$ & $2.0 \times 10^{-3}$ & & $6.3 \times 10^{-1}$ & $1.0 \times 10^{-3}$ & $1.9 \times 10^{\circ 2}$ & & $8.1 \times 10^{0}$ & $1.9 \times 10^{-1}$ & $9.2 \times 10^{0}$ \\
\hline 1991 & $2.5 \times 10^{-5}$ & $6.2 \times 10^{-3}$ & $3.2 \times 10^{-3}$ & $1.8 \times 10^{-1}$ & $9.9 \times 10^{-6}$ & $2.9 \times 10^{-2}$ & $4.2 \times 10^{-3}$ & & $1.4 \times 10^{-1}$ & $3,8 \times 10^{-4}$ & $9.6 \times 10^{-3}$ & & $6.3 \times 10^{\circ}$ & $1.0 \times 10^{-1}$ & $6.8 \times 10^{0}$ \\
\hline 1992 & $2.6 \times 10^{-6}$ & $1.9 \times 10^{-3}$ & $2.4 \times 10^{-3}$ & $6.3 \times 10^{-2}$ & $3.3 \times 10^{-5}$ & $2.2 \times 10^{-2}$ & $2.3 \times 10^{3}$ & $7.5 \times 10^{-3}$ & $2.2 \times 10^{-1}$ & $1.5 \times 10^{-6}$ & $4.9 \times 10^{-3}$ & & $4.6 \times 10^{0}$ & $5.8 \times 10^{-2}$ & $5.0 \times 10^{0}$ \\
\hline 1993 & $4.2 \times 10^{-8}$ & $1.7 \times 10^{-4}$ & $6.1 \times 10^{3}$ & $9.0 \times 10^{-3}$ & $4.9 \times 10^{-5}$ & $2.7 \times 10^{-2}$ & $5.6 \times 10^{-3}$ & & $4.3 \times 10^{-1}$ & $3.4 \times 10^{-6}$ & $5.5 \times 10^{-3}$ & & $6.2 \times 10^{0}$ & $7.1 \times 10^{-2}$ & $6.8 \times 10^{0}$ \\
\hline 1994 & $4.4 \times 10^{-5}$ & $3.7 \times 10^{-4}$ & $1.5 \times 10^{-3}$ & $6.9 \times 10^{-2}$ & $2.3 \times 10^{-5}$ & $1.1 \times 10^{-2}$ & $1.6 \times 10^{-3}$ & & $4.4 \times 10^{-1}$ & $1.4 \times 10^{-8}$ & $5.1 \times 10^{-3}$ & & $5.0 \times 10^{0}$ & $8.3 \times 10^{-2}$ & $5.6 \times 10^{0}$ \\
\hline 1995 & $4.1 \times 10^{-4}$ & & $1.4 \times 10^{-1}$ & $8.5 \times 10^{-2}$ & $6.8 \times 10^{-3}$ & $5.8 \times 10^{.3}$ & $3.4 \times 10^{-4}$ & & $2.5 \times 10^{-4}$ & $1.5 \times 10^{-4}$ & $1.3 \times 10^{-2}$ & & $2.5 \times 10^{0}$ & $5.8 \times 10^{-2}$ & $3.1 \times 10^{\circ}$ \\
\hline 1996 & $6.7 \times 10^{-5}$ & $8.1 \times 10^{-2}$ & $4.7 \times 10^{-2}$ & $1.9 \times 10^{-1}$ & $2.9 \times 10^{-5}$ & $2.5 \times 10^{-3}$ & $1.3 \times 10^{-2}$ & & $1.9 \times 10^{-1}$ & $6.0 \times 10^{-2}$ & $7.5 \times 10^{-3}$ & & $1.9 \times 10^{0}$ & $6.3 \times 10^{-2}$ & $2.6 \times 10^{\circ}$ \\
\hline Total & $6.5 \times 10^{-1}$ & $3.0 \times 10^{1}$ & $3.4 \times 10^{1}$ & $1.0 \times 10^{2}$ & $8.3 \times 10^{2}$ & $1.2 \times 10^{0}$ & $8.9 \times 10^{0}$ & $1.9 \times 10^{2}$ & $7.1 \times 10^{2}$ & $1.2 \times 10^{2}$ & $6.3 \times 10^{0}$ & $6.5 \times 10^{0}$ & $1.1 \times 10^{3}$ & $3.2 \times 10^{1}$ & $3.2 \times 10^{3}$ \\
\hline
\end{tabular}




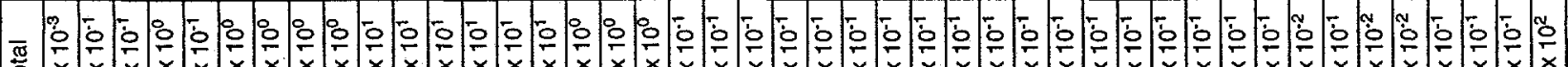

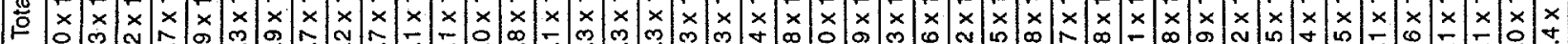

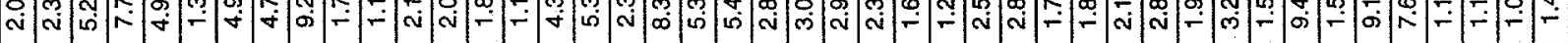

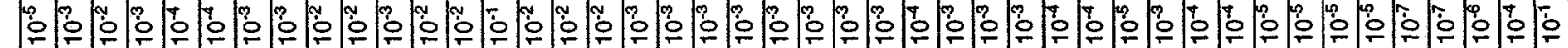

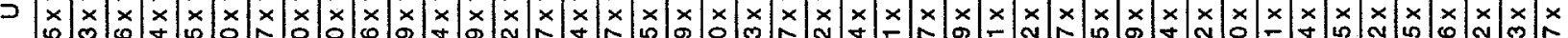

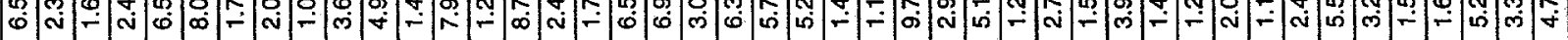

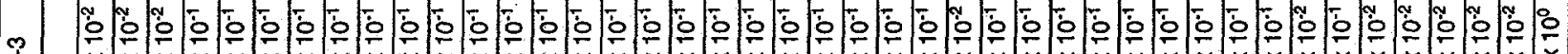

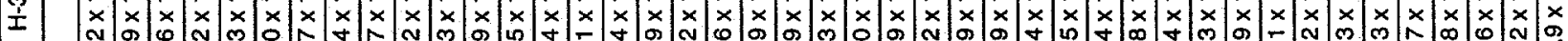
N

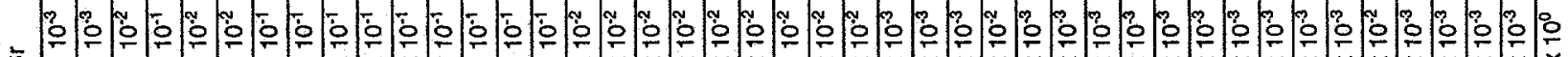

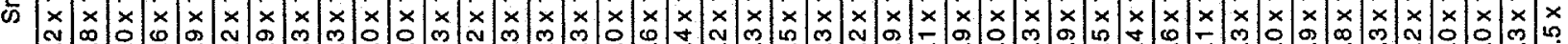

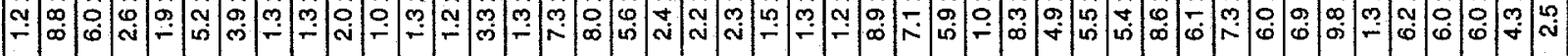

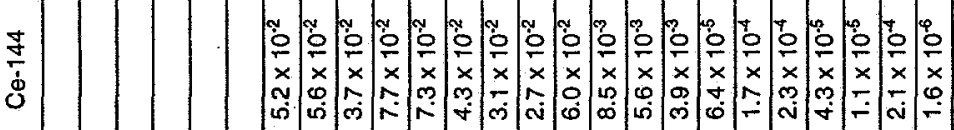

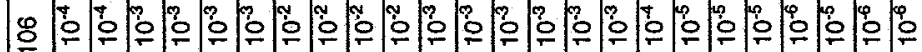

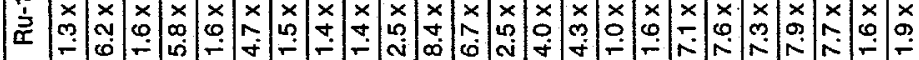

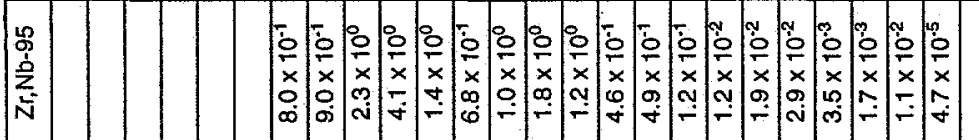

$\infty$
0
0
0
0
0
0
0
0

.

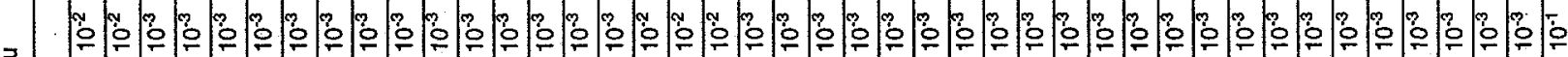

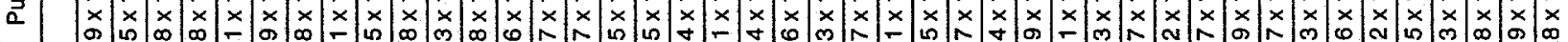

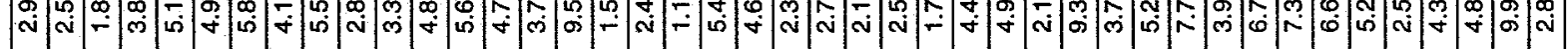

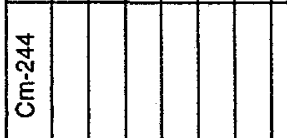

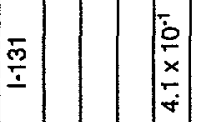

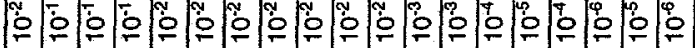

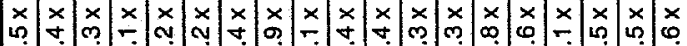

$\frac{10}{2}$

ڤ.

向

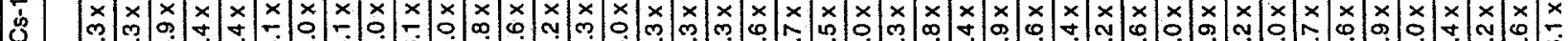
O

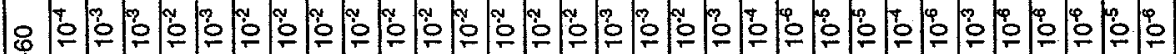

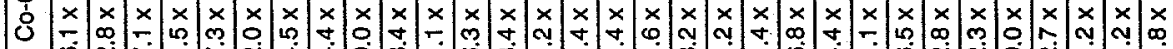

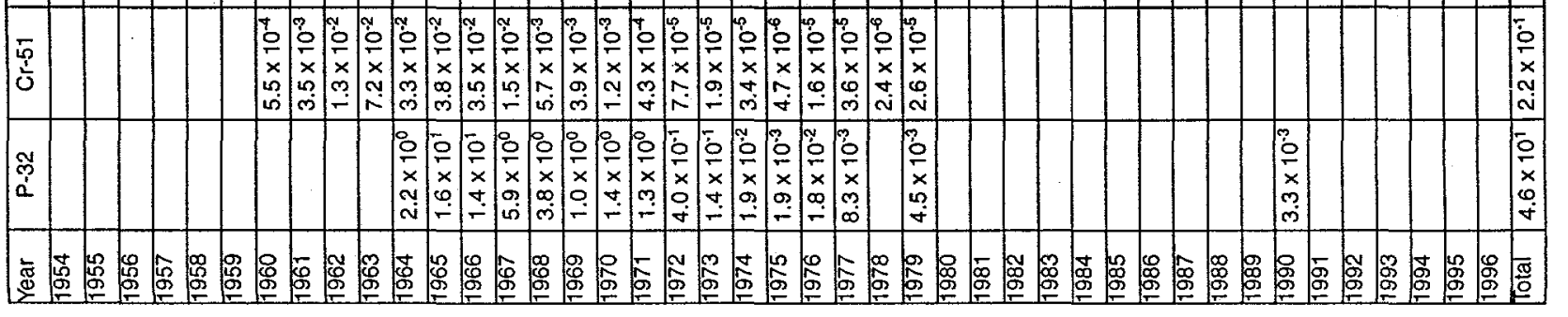




\begin{tabular}{|c|c|c|c|c|c|c|c|c|c|c|c|c|c|c|c|}
\hline Year & P-32 & $\mathrm{Cr}-51$ & Co-60 & $2 n-65$ & Cs.137 & $\mid-131$ & $\mathrm{Cm}-244$ & $\mathrm{Pu}$ & $\mathrm{Zr}, \mathrm{Nb}-95$ & Ru-106 & $\mathrm{C}_{\theta-144}$ & $\mathrm{Sr}$ & $\mathrm{H}-3$ & $\mathrm{U}$ & Total \\
\hline 1954 & & & & & & & & & & & & & & & \\
\hline 1955 & & & & & & & & & & & & & & & \\
\hline 1956 & & & & & & & & & & & & & & & \\
\hline 1957 & & & & & & & & & & & & & & & \\
\hline 1958 & & & & & & & & & & & & & & & \\
\hline 1959 & & & & & & & & & & & & & & & \\
\hline 1960 & & & & & & & & & & & & & & & \\
\hline 1961 & & & & & & & & & & & & & & & \\
\hline 1962 & & & & & & & & & & & & & & & \\
\hline 1963 & & & & & & & & & & & & & & & \\
\hline 1964 & & & & & & & & & & & & & & & \\
\hline 1965 & $2.8 \times 10^{-3}$ & $2.7 \times 10^{-3}$ & $8.0 \times 10^{-3}$ & $3.5 \times 10^{-3}$ & $1.7 \times 10^{-2}$ & $7.3 \times 10^{-3}$ & & $1.4 \times 10^{-3}$ & $5.9 \times 10^{-4}$ & $2.3 \times 10^{-3}$ & $1.8 \times 10^{-2}$ & $3.3 \times 10^{-2}$ & $1.9 \times 10^{-1}$ & $6.0 \times 10^{-3}$ & $2.9 \times 10^{-1}$ \\
\hline 1966 & $3.3 \times 10^{-3}$ & $3.4 \times 10^{-3}$ & $5.8 \times 10^{-3}$ & $4.5 \times 10^{-3}$ & $2.9 \times 10^{-2}$ & $6.2 \times 10^{-3}$ & & $2.7 \times 10^{-3}$ & $1.2 \times 10^{-3}$ & $1.2 \times 10^{-3}$ & $1.8 \times 10^{-2}$ & $4.4 \times 10^{-2}$ & $2.3 \times 10^{-1}$ & $4.5 \times 10^{-2}$ & $3.9 \times 10^{-1}$ \\
\hline 1967 & $1.1 \times 10^{-3}$ & $1.1 \times 10^{-3}$ & $1.6 \times 10^{-3}$ & $3.6 \times 10^{-3}$ & $5.1 \times 10^{-2}$ & $2.4 \times 10^{-2}$ & & $2.5 \times 10^{-3}$ & $1.7 \times 10^{-3}$ & $1.5 \times 10^{-3}$ & $1.2 \times 10^{-2}$ & $9.3 \times 10^{-2}$ & $1.6 \times 10^{-1}$ & $5.5 \times 10^{-2}$ & $4.1 \times 10^{-1}$ \\
\hline 1968 & $8.7 \times 10^{-4}$ & $5.2 \times 10^{-4}$ & $2.3 \times 10^{-3}$ & $3.0 \times 10^{-3}$ & $3.8 \times 10^{-2}$ & $2.2 \times 10^{.2}$ & & $2.5 \times 10^{-3}$ & $1.3 \times 10^{-3}$ & $1.9 \times 10^{-3}$ & $3.3 \times 10^{-2}$ & $4.2 \times 10^{-2}$ & $1.9 \times 10^{-1}$ & $4.8 \times 10^{-2}$ & $3.9 \times 10^{-1}$ \\
\hline 1969 & $2.0 \times 10^{-4}$ & $3.1 \times 10^{-4}$ & $2.0 \times 10^{-4}$ & $7.6 \times 10^{-4}$ & $1.4 \times 10^{-2}$ & $8.6 \times 10^{-3}$ & $2.6 \times 10^{-3}$ & $1.7 \times 10^{-3}$ & $4.4 \times 10^{-4}$ & $4.0 \times 10^{-4}$ & $4.0 \times 10^{-3}$ & $2.1 \times 10^{-2}$ & $1.5 \times 10^{-1}$ & $1.1 \times 10^{-2}$ & $2.2 \times 10^{-1}$ \\
\hline 1970 & $1.7 \times 10^{-4}$ & $5.9 \times 10^{-5}$ & $1.3 \times 10^{-4}$ & $2.1 \times 10^{-4}$ & $1.1 \times 10^{-2}$ & $3.1 \times 10^{-3}$ & & $2.6 \times 10^{-3}$ & $2.8 \times 10^{-4}$ & $3.8 \times 10^{-5}$ & $1.6 \times 10^{-3}$ & $1.4 \times 10^{-2}$ & $6.9 \times 10^{-2}$ & $4.7 \times 10^{-3}$ & $1.1 \times 10^{-1}$ \\
\hline 1971 & $1.2 \times 10^{-4}$ & $1.7 \times 10^{-5}$ & $2.2 \times 10^{-3}$ & $4.4 \times 10^{-4}$ & $1.0 \times 10^{-3}$ & $1.3 \times 10^{-3}$ & $1.4 \times 10^{-2}$ & $3.3 \times 10^{-3}$ & $5.5 \times 10^{-5}$ & $1.3 \times 10^{-5}$ & $8.8 \times 10^{-4}$ & $7.8 \times 10^{-3}$ & $4.4 \times 10^{-2}$ & $1.5 \times 10^{-3}$ & $7.7 \times 10^{-2}$ \\
\hline 1972 & $6.5 \times 10^{-5}$ & $5.0 \times 10^{-6}$ & $1.4 \times 10^{-4}$ & & $6.8 \times 10^{-4}$ & $1.3 \times 10^{-3}$ & $1.9 \times 10^{-3}$ & $9.2 \times 10^{-3}$ & $9.8 \times 10^{-6}$ & $2.4 \times 10^{-5}$ & $2.5 \times 10^{-5}$ & $5.8 \times 10^{-3}$ & $8.6 \times 10^{-2}$ & $2.7 \times 10^{-3}$ & $1.1 \times 10^{-1}$ \\
\hline 1973 & $3.4 \times 10^{-5}$ & $1.9 \times 10^{-6}$ & $2.5 \times 10^{-5}$ & & $5.7 \times 10^{-4}$ & $2.6 \times 10^{-4}$ & $3.0 \times 10^{-4}$ & $6.1 \times 10^{-3}$ & $2.2 \times 10^{-5}$ & $3.5 \times 10^{-5}$ & $1.0 \times 10^{-4}$ & $8.0 \times 10^{-3}$ & $1.6 \times 10^{-1}$ & $1.8 \times 10^{-3}$ & $1.8 \times 10^{-1}$ \\
\hline 1974 & $3.7 \times 10^{-6}$ & $2.7 \times 10^{-6}$ & $9.3 \times 10^{-7}$ & $6.8 \times 10^{.5}$ & $9.5 \times 10^{-4}$ & $2.4 \times 10^{-5}$ & & $2.4 \times 10^{-3}$ & $2.8 \times 10^{-5}$ & $3.0 \times 10^{-6}$ & $1.1 \times 10^{-4}$ & $6.6 \times 10^{-3}$ & $1.4 \times 10^{-1}$ & $2.9 \times 10^{-3}$ & $1.5 \times 10^{-1}$ \\
\hline 1975 & $3.8 \times 10^{-7}$ & $3.7 \times 10^{-7}$ & $6.1 \times 10^{-6}$ & $7.1 \times 10^{-7}$ & $3.5 \times 10^{-4}$ & $1.5 \times 10^{4}$ & & $2.1 \times 10^{-3}$ & $3.4 \times 10^{6}$ & $3.0 \times 10^{-5}$ & $2.0 \times 10^{-5}$ & $4.3 \times 10^{-3}$ & $9.2 \times 10^{-2}$ & $2.7 \times 10^{-3}$ & $1.0 \times 10^{-1}$ \\
\hline 1976 & $2.8 \times 10^{-6}$ & $1.0 \times 10^{-6}$ & $1.2 \times 10^{-6}$ & & $1.2 \times 10^{-4}$ & $2.2 \times 10^{-6}$ & & $8.3 \times 10^{-4}$ & $1.3 \times 10^{-6}$ & $4.8 \times 10^{-7}$ & $4.1 \times 10^{-6}$ & $3.0 \times 10^{-3}$ & $8.6 \times 10^{-2}$ & $1.9 \times 10^{-3}$ & $9.2 \times 10^{-2}$ \\
\hline 1977 & $1.9 \times 10^{-6}$ & $3.3 \times 10^{-6}$ & $1.1 \times 10^{-4}$ & & $3.7 \times 10^{-4}$ & $1.9 \times 10^{-5}$ & & $1.5 \times 10^{-3}$ & $1.3 \times 10^{-5}$ & $8.8 \times 10^{-7}$ & $1.1 \times 10^{-4}$ & $3.9 \times 10^{-3}$ & $1.1 \times 10^{-1}$ & $8.0 \times 10^{-4}$ & $1.2 \times 10^{-1}$ \\
\hline 1978 & & $1.6 \times 10^{-7}$ & $3.4 \times 10^{-7}$ & & $1.3 \times 10^{-4}$ & $1.1 \times 10^{-6}$ & & $8.3 \times 10^{-4}$ & $3.9 \times 10^{-8}$ & & $6.3 \times 10^{-7}$ & $2.2 \times 10^{-3}$ & $7.9 \times 10^{-2}$ & $4.4 \times 10^{-4}$ & $8.3 \times 10^{-2}$ \\
\hline 1979 & $2.4 \times 10^{-5}$ & $1.5 \times 10^{-6}$ & $2.5 \times 10^{-4}$ & & $8.7 \times 10^{-5}$ & & & $8.8 \times 10^{-4}$ & & & & $1.6 \times 10^{-3}$ & $4.3 \times 10^{-2}$ & $3.5 \times 10^{-4}$ & $4.6 \times 10^{-2}$ \\
\hline 1980 & & & $1.1 \times 10^{6}$ & & $7.6 \times 10^{-5}$ & & & $6.6 \times 10^{-4}$ & & & & $1.5 \times 10^{-3}$ & $4.1 \times 10^{-2}$ & $1.2 \times 10^{-3}$ & $4.4 \times 10^{-2}$ \\
\hline 1981 & & & $4.3 \times 10^{-7}$ & & $2.7 \times 10^{-4}$ & & & $2.3 \times 10^{-3}$ & & & & $3.4 \times 10^{-3}$ & $1.0 \times 10^{-1}$ & $2.8 \times 10^{-3}$ & $1.1 \times 10^{-1}$ \\
\hline 1982 & & & $1.2 \times 10^{-7}$ & & $1.2 \times 10^{-4}$ & & & $1.6 \times 10^{-3}$ & & & & $1.8 \times 10^{-3}$ & $8.5 \times 10^{-2}$ & $4.1 \times 10^{-4}$ & $8.9 \times 10^{-2}$ \\
\hline 1983 & & & $3.1 \times 10^{-6}$ & & $1.6 \times 10^{-4}$ & & & $1.9 \times 10^{-3}$ & & & & $2.8 \times 10^{-3}$ & $1.4 \times 10^{-1}$ & $2.5 \times 10^{-4}$ & $1.5 \times 10^{-1}$ \\
\hline 1984 & & & $2.5 \times 10^{-7}$ & & $1.3 \times 10^{-4}$ & & & $4.1 \times 10^{-3}$ & & & & $1.5 \times 10^{-3}$ & $6.3 \times 10^{-2}$ & $7.0 \times 10^{-5}$ & $6.9 \times 10^{-2}$ \\
\hline 1985 & & & & & $1.5 \times 10^{-4}$ & & & $2.6 \times 10^{-3}$ & & & & $2.4 \times 10^{-3}$ & $1.4 \times 10^{-1}$ & $2.9 \times 10^{-5}$ & $1.5 \times 10^{-1}$ \\
\hline 1986 & & & & & $1.8 \times 10^{-4}$ & & & $3.6 \times 10^{-3}$ & & & & $3.7 \times 10^{-3}$ & $1.7 \times 10^{-1}$ & $1.0 \times 10^{-3}$ & $1.8 \times 10^{-1}$ \\
\hline 1987 & & & & & $4.1 \times 10^{-4}$ & & & $4.8 \times 10^{-3}$ & & & & $2.4 \times 10^{-3}$ & $8.8 \times 10^{-2}$ & $7.9 \times 10^{-5}$ & $9.6 \times 10^{-2}$ \\
\hline 1988 & & & & & $1.1 \times 10^{-3}$ & & & $2.6 \times 10^{-3}$ & & & & $3.1 \times 10^{-3}$ & $1.3 \times 10^{-1}$ & $1.4 \times 10^{-4}$ & $1.4 \times 10^{-1}$ \\
\hline 1989 & & & & & $3.5 \times 10^{-4}$ & & & $5.9 \times 10^{-3}$ & $1.8 \times 10^{-8}$ & $9.4 \times 10^{-6}$ & $8.5 \times 10^{-8}$ & $3.3 \times 10^{-3}$ & $1.0 \times 10^{-1}$ & $9.4 \times 10^{6}$ & $1.1 \times 10^{-1}$ \\
\hline 1990 & $9.7 \times 10^{-7}$ & & & & $4.4 \times 10^{-5}$ & & & $5.1 \times 10^{-3}$ & & & & $3.0 \times 10^{-3}$ & $5.2 \times 10^{-2}$ & $1.7 \times 10^{-5}$ & $6.0 \times 10^{-2}$ \\
\hline 1991 & & & & & $1.9 \times 10^{-5}$ & & & $3.6 \times 10^{-3}$ & & क. & & $3.4 \times 10^{-3}$ & $7.2 \times 10^{-2}$ & $3.1 \times 10^{-5}$ & $7.9 \times 10^{-2}$ \\
\hline 1992 & & & & & $1.2 \times 10^{-4}$ & & & $4.5 \times 10^{-3}$ & & & & $7.0 \times 10^{-3}$ & $5.8 \times 10^{-2}$ & $2.9 \times 10^{-5}$ & $7.0 \times 10^{-2}$ \\
\hline 1993 & & & & & $1.8 \times 10^{-4}$ & & & $1.7 \times 10^{-3}$ & & & & $2.6 \times 10^{-3}$ & $3.3 \times 10^{-2}$ & $1.0 \times 10^{-7}$ & $3.7 \times 10^{-2}$ \\
\hline 1994 & & & & & $5.5 \times 10^{-4}$ & & & $3.3 \times 10^{-3}$ & & & & $2.9 \times 10^{-3}$ & $3.8 \times 10^{-2}$ & $1.3 \times 10^{-7}$ & $4.5 \times 10^{-2}$ \\
\hline 1995 & & & $3.1 \times 10^{-6}$ & & $5.3 \times 10^{-4}$ & & $4.4 \times 10^{-7}$ & $3.6 \times 10^{-3}$ & & & & $2.8 \times 10^{-3}$ & $3.6 \times 10^{-2}$ & $4.1 \times 10^{-6}$ & $4.3 \times 10^{-2}$ \\
\hline 1996 & & & & & $6.0 \times 10^{-4}$ & & $2.1 \times 10^{-6}$ & $9.6 \times 10^{-3}$ & & & & $2.6 \times 10^{-3}$ & $4.2 \times 10^{-2}$ & $3.3 \times 10^{-4}$ & $5.5 \times 10^{-2}$ \\
\hline Total & $8.7 \times 10^{-3}$ & $8.2 \times 10^{-3}$ & $2.1 \times 10^{-2}$ & $1.6 \times 10^{-2}$ & $1.7 \times 10^{-1}$ & $7.4 \times 10^{-2}$ & $1.9 \times 10^{-2}$ & $1.0 \times 10^{-1}$ & $5.6 \times 10^{-3}$ & $7.5 \times 10^{-3}$ & $8.8 \times 10^{-2}$ & $3.4 \times 10^{-1}$ & $3.2 \times 10^{0}$ & $1.9 \times 10^{-1}$ & $4.3 \times 10^{0}$ \\
\hline
\end{tabular}




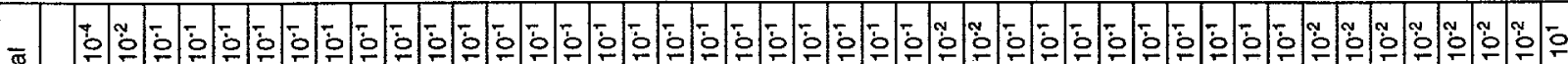

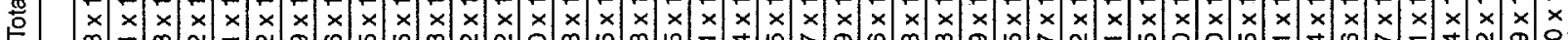
$\infty$ 至

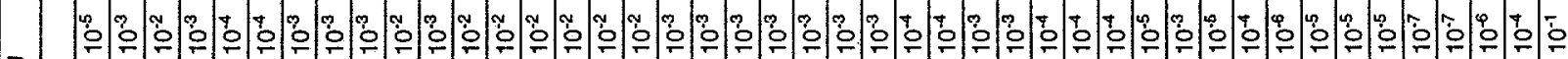

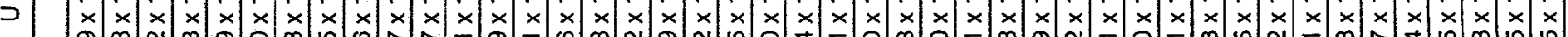

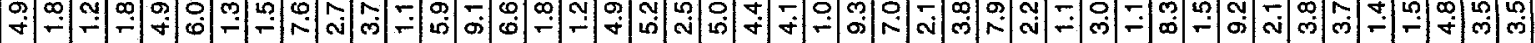

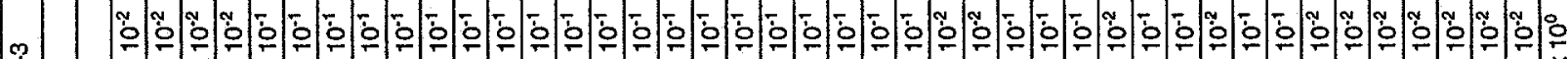

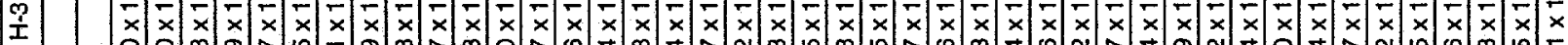

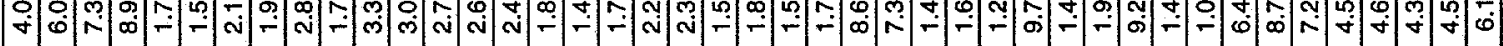

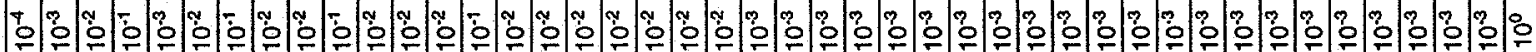

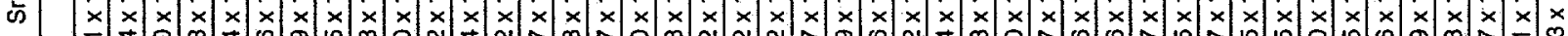
ம)

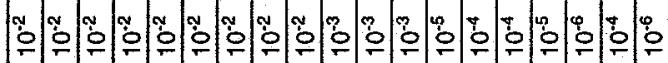

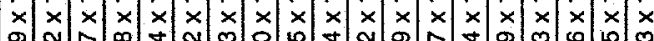

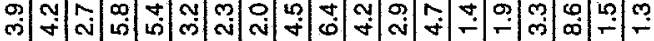

$\infty$
0
0
$x$
0
0

m.

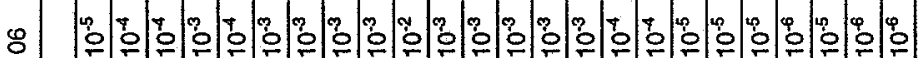

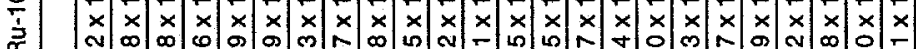

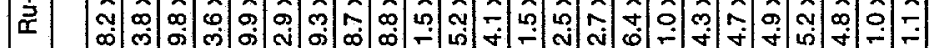

$\mid \begin{aligned} & 9 \\ & 0 \\ & x \\ & \sim\end{aligned}$

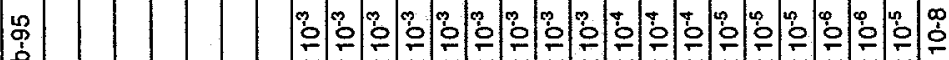

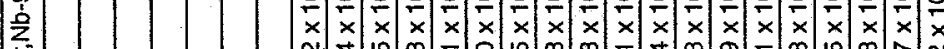

N

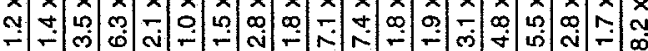

竞

i.

(1)

1

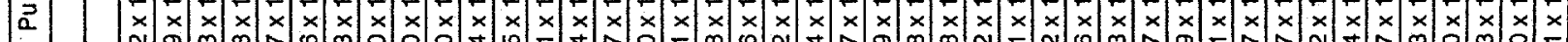

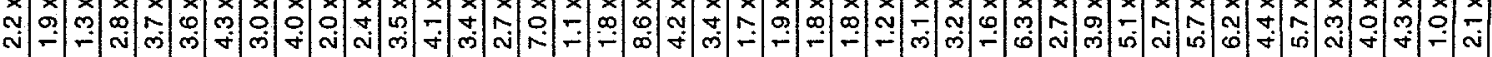

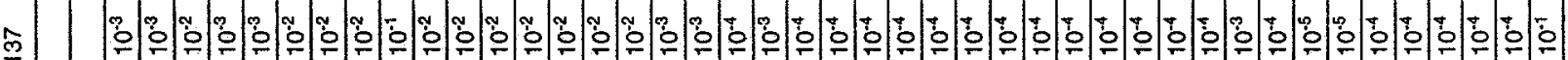

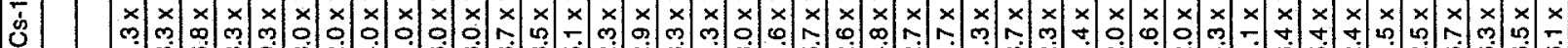

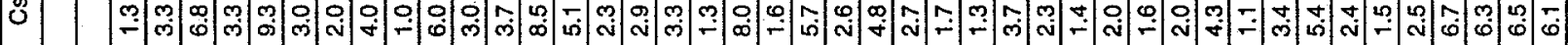

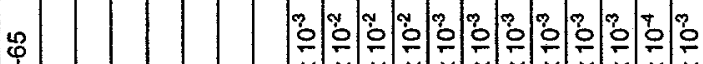




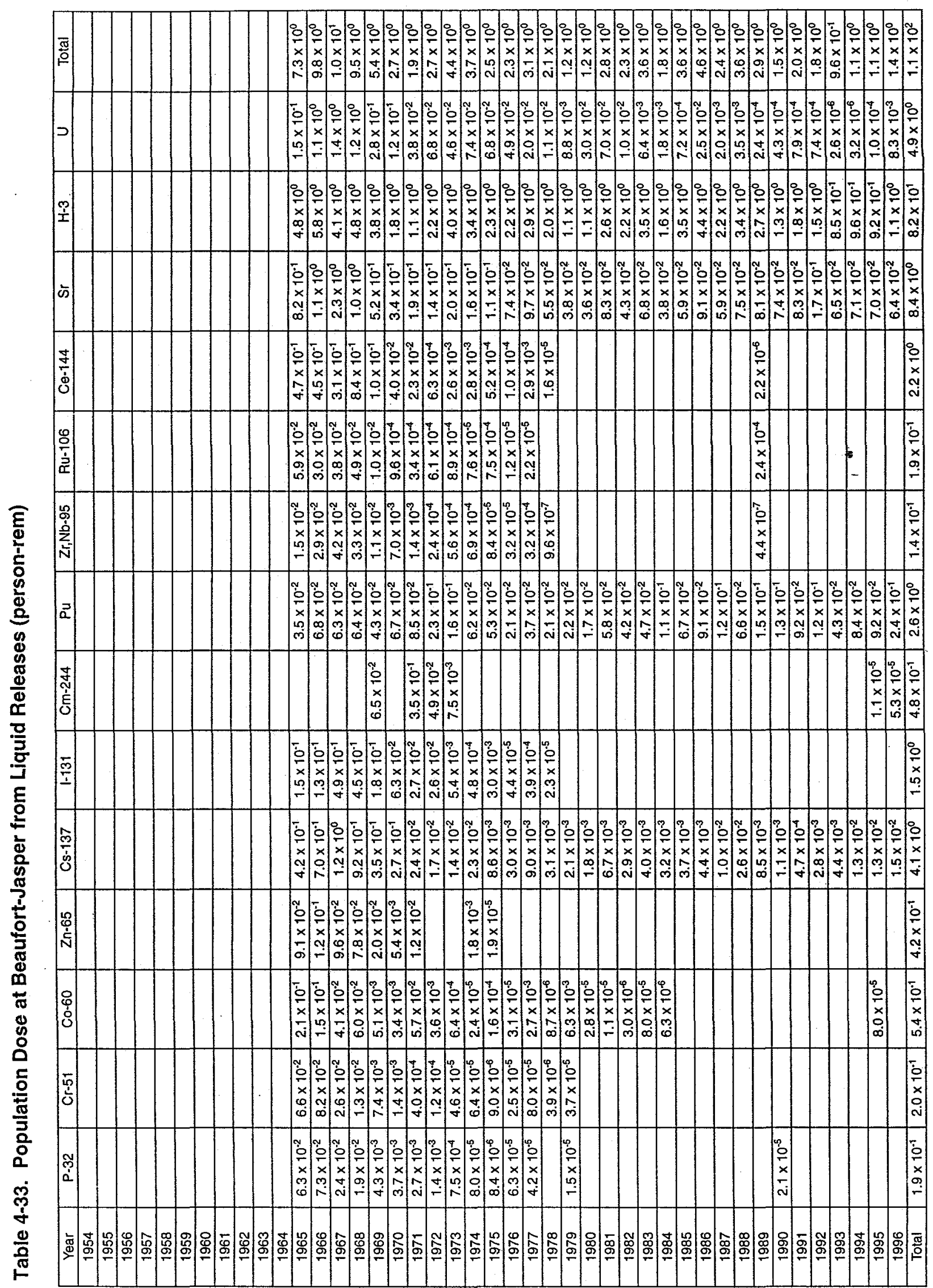


$\mathscr{\infty}$ | Table 4-34. Population Dose at Port Wentworth from Liquid Releases (person-rem)

\begin{tabular}{|c|c|c|c|c|c|c|c|c|c|c|c|c|c|c|c|}
\hline Year & P.32 & Cr-51 & Co-60 & $Z n-65$ & Cs-137 & $1-1,31$ & $\mathrm{Cm}-244$ & $\mathrm{Pu}$ & $\mathrm{Zr}, \mathrm{Nb}-95$ & Ru-106 & $\mathrm{Ce}_{\mathrm{e}-144}$ & $\mathrm{Sr}$ & $\mathrm{H}-3$ & $u$ & Total \\
\hline 1954 & & & $1.0 \times 10^{-3}$ & & & & & & & $6.3 \times 10^{-4}$ & & $4.1 \times 10^{-3}$ & & $3.6 \times 10^{-4}$ & $6.1 \times 10^{-3}$ \\
\hline 1955 & & & $4.8 \times 10^{-3}$ & & $9.7 \times 10^{-3}$ & & & $1.7 \times 10^{-1}$ & & $2.9 \times 10^{-3}$ & & $3.0 \times 10^{-2}$ & $3.0 \times 10^{-1}$ & $1.3 \times 10^{-2}$ & $5.3 \times 10^{-1}$ \\
\hline 1956 & & & $1.2 \times 10^{-2}$ & & $2.5 \times 10^{-2}$ & & & $1.4 \times 10^{-1}$ & & $7.5 \times 10^{-3}$ & & $2.0 \times 10^{-1}$ & $4.6 \times 10^{-1}$ & $9.2 \times 10^{-2}$ & $9.4 \times 10^{-1}$ \\
\hline 1957 & & & $2.5 \times 10^{-2}$ & & $5.1 \times 10^{-1}$ & $1.4 \times 10^{0}$ & & $1.0 \times 10^{-2}$ & & $2.7 \times 10^{-2}$ & & $8.7 \times 10^{-1}$ & $5.6 \times 10^{-1}$ & $1.3 \times 10^{-2}$ & $3.4 \times 10^{0}$ \\
\hline 1958 & & & $1.2 \times 10^{-2}$ & & $2.5 \times 10^{-2}$ & & & $2.1 \times 10^{-2}$ & & $7.6 \times 10^{-3}$ & & $6.3 \times 10^{-2}$ & $6.8 \times 10^{-1}$ & $3.6 \times 10^{-3}$ & $8.1 \times 10^{-1}$ \\
\hline 1959 & & & $3.4 \times 10^{-2}$ & & $7.0 \times 10^{-2}$ & & & $2.9 \times 10^{-2}$ & & $2.2 \times 10^{-2}$ & & $1.8 \times 10^{-1}$ & $1.3 \times 10^{0}$ & $4.4 \times 10^{-3}$ & $1.6 \times 10^{0}$ \\
\hline 1960 & & $5.0 \times 10^{-4}$ & $7.5 \times 10^{-2}$ & $2.2 \times 10^{-2}$ & $2.3 \times 10^{-1}$ & $3.3 \times 10^{-4}$ & & $2.7 \times 10^{-2}$ & $9.1 \times 10^{-3}$ & $7.1 \times 10^{-2}$ & $2.9 \times 10^{-1}$ & $1.3 \times 10^{0}$ & $1.1 \times 10^{0}$ & $9.3 \times 10^{-3}$ & $3.5 \times 10^{0}$ \\
\hline 1961 & & $3.3 \times 10^{-3}$ & $7.5 \times 10^{-2}$ & $1.3 \times 10^{-1}$ & $1.5 \times 10^{-1}$ & $4.9 \times 10^{-1}$ & & $3.3 \times 10^{-2}$ & $1.0 \times 10^{-2}$ & $6.6 \times 10^{-2}$ & $3.1 \times 10^{-1}$ & $4.4 \times 10^{-1}$ & $1.6 \times 10^{0}$ & $1.1 \times 10^{-2}$ & $3.3 \times 10^{0}$ \\
\hline 1962 & & $1.2 \times 10^{-2}$ & $1.5 \times 10^{-1}$ & $2.1 \times 10^{-1}$ & $3.0 \times 10^{-1}$ & $1.5 \times 10^{0}$ & & $2.3 \times 10^{-2}$ & $2.6 \times 10^{-2}$ & $6.7 \times 10^{-2}$ & $2.1 \times 10^{-1}$ & $4.6 \times 10^{-1}$ & $1.4 \times 10^{0}$ & $5.6 \times 10^{-2}$ & $4.4 \times 10^{0}$ \\
\hline 1963 & & $6.7 \times 10^{-2}$ & $5.8 \times 10^{-2}$ & $2.1 \times 10^{-1}$ & $7.5 \times 10^{-1}$ & $7.5 \times 10^{-1}$ & & $3.1 \times 10^{-2}$ & $4.6 \times 10^{-2}$ & $1.2 \times 10^{-1}$ & $4.3 \times 10^{-1}$ & $6.9 \times 10^{-1}$ & $2.2 \times 10^{0}$ & $2.0 \times 10^{-1}$ & $5.6 \times 10^{0}$ \\
\hline 1964 & $4.6 \times 10^{-3}$ & $3.1 \times 10^{-2}$ & $1.9 \times 10^{-2}$ & $6.6 \times 10^{-2}$ & $4.6 \times 10^{-1}$ & $7.7 \times 10^{-2}$ & & $1.6 \times 10^{-2}$ & $1.5 \times 10^{-2}$ & $4.0 \times 10^{-2}$ & $4.1 \times 10^{-1}$ & $3.5 \times 10^{-1}$ & $1.3 \times 10^{0}$ & $2.7 \times 10^{-2}$ & $2.8 \times 10^{0}$ \\
\hline 1965 & $3.3 \times 10^{-2}$ & $3.5 \times 10^{-2}$ & $1.1 \times 10^{-1}$ & $4.8 \times 10^{-2}$ & $2.3 \times 10^{-1}$ & $7.7 \times 10^{-2}$ & & $1.8 \times 10^{-2}$ & $7.7 \times 10^{-3}$ & $3.1 \times 10^{-2}$ & $2.4 \times 10^{-1}$ & $4.3 \times 10^{-1}$ & $2.5 \times 10^{0}$ & $7.8 \times 10^{-2}$ & $3.8 \times 10^{0}$ \\
\hline 1966 & $2.8 \times 10^{-2}$ & $3.2 \times 10^{-2}$ & $5.7 \times 10^{-2}$ & $4.6 \times 10^{-2}$ & $2.8 \times 10^{-1}$ & $4.9 \times 10^{-2}$ & & $2.7 \times 10^{-2}$ & $1.1 \times 10^{-2}$ & $1.2 \times 10^{-2}$ & $1.7 \times 10^{-1}$ & $4.2 \times 10^{-1}$ & $2.3 \times 10^{0}$ & $4.4 \times 10^{-1}$ & $3.9 \times 10^{0}$ \\
\hline 1967 & $1.2 \times 10^{-2}$ & $1.3 \times 10^{-2}$ & $2.0 \times 10^{-2}$ & $4.7 \times 10^{-2}$ & $6.4 \times 10^{-1}$ & $2.4 \times 10^{-1}$ & & $3.2 \times 10^{-2}$ & $2.1 \times 10^{-2}$ & $1.9 \times 10^{-2}$ & $1.5 \times 10^{-1}$ & $1.1 \times 10^{0}$ & $2.0 \times 10^{0}$ & $6.7 \times 10^{-1}$ & $5.0 \times 10^{0}$ \\
\hline 1968 & $7.9 \times 10^{-3}$ & $5.2 \times 10^{-3}$ & $2.4 \times 10^{-2}$ & $3.2 \times 10^{-2}$ & $3.9 \times 10^{-1}$ & $1.8 \times 10^{-1}$ & & $2.7 \times 10^{-2}$ & $1.3 \times 10^{-2}$ & $2.0 \times 10^{-2}$ & $3.4 \times 10^{-1}$ & $4.3 \times 10^{-1}$ & $2.0 \times 10^{0}$ & $4.9 \times 10^{-1}$ & $4.0 \times 10^{0}$ \\
\hline 1969 & $2.1 \times 10^{-3}$ & $3.6 \times 10^{-3}$ & $2.4 \times 10^{-3}$ & $9.5 \times 10^{-3}$ & $1.7 \times 10^{-1}$ & $8.3 \times 10^{-2}$ & $3.0 \times 10^{-2}$ & $2.1 \times 10^{-2}$ & $5.2 \times 10^{3}$ & $4.9 \times 10^{-3}$ & $4.8 \times 10^{-2}$ & $2.5 \times 10^{-1}$ & $1.8 \times 10^{0}$ & $1.3 \times 10^{-1}$ & $2.6 \times 10^{0}$ \\
\hline 1970 & $2.9 \times 10^{-3}$ & $1.1 \times 10^{-3}$ & $2.7 \times 10^{-3}$ & $4.2 \times 10^{-3}$ & $2.2 \times 10^{-1}$ & $4.9 \times 10^{-2}$ & & $5.4 \times 10^{-2}$ & $5.5 \times 10^{-3}$ & $7.7 \times 10^{-4}$ & $3.1 \times 10^{-2}$ & $2.7 \times 10^{-1}$ & $1.4 \times 10^{0}$ & $9.2 \times 10^{-2}$ & $2.1 \times 10^{0}$ \\
\hline 1971 & $2.7 \times 10^{-3}$ & $4.0 \times 10^{4}$ & $5.5 \times 10^{-2}$ & $1.1 \times 10^{-2}$ & $2.5 \times 10^{-2}$ & $2.6 \times 10^{-2}$ & $3.4 \times 10^{-1}$ & $8.4 \times 10^{-2}$ & $1.3 \times 10^{-3}$ & $3.3 \times 10^{-4}$ & $2.2 \times 10^{-2}$ & $1.9 \times 10^{-1}$ & $1.1 \times 10^{\circ}$ & $3.6 \times 10^{-2}$ & $1.9 \times 10^{0}$ \\
\hline 1972 & $8.3 \times 10^{-4}$ & $7.1 \times 10^{-5}$ & $2.0 \times 10^{-3}$ & & $1.0 \times 10^{-2}$ & $1.5 \times 10^{-2}$ & $2.8 \times 10^{-2}$ & $1.4 \times 10^{-1}$ & $1.4 \times 10^{-4}$ & $3.6 \times 10^{-4}$ & $3.6 \times 10^{-4}$ & $8.2 \times 10^{-2}$ & $1.3 \times 10^{0}$ & $3.9 \times 10^{-2}$ & $1.6 \times 10^{0}$ \\
\hline 1973 & $3.1 \times 10^{-4}$ & $1.9 \times 10^{-5}$ & $2.7 \times 10^{-4}$ & & $6.0 \times 10^{-3}$ & $2.2 \times 10^{-3}$ & $3.1 \times 10^{-3}$ & $6.6 \times 10^{-2}$ & $2.3 \times 10^{-4}$ & $3.8 \times 10^{-4}$ & $1.1 \times 10^{-3}$ & $8.2 \times 10^{-2}$ & $1.7 \times 10^{0}$ & $1.9 \times 10^{-2}$ & $1.9 \times 10^{\circ}$ \\
\hline 1974 & $4.1 \times 10^{-5}$ & $3.3 \times 10^{-6}$ & $1.2 \times 10^{-5}$ & $9.1 \times 10^{-4}$ & $1.2 \times 10^{-2}$ & $2.4 \times 10^{-4}$ & & $3.2 \times 10^{-2}$ & $3.5 \times 10^{-4}$ & $3.9 \times 10^{-5}$ & $1.4 \times 10^{-3}$ & $8.4 \times 10^{-2}$ & $1.8 \times 10^{0}$ & $3.7 \times 10^{-2}$ & $2.0 \times 10^{0}$ \\
\hline 1975 & $4.1 \times 10^{-6}$ & $4.5 \times 10^{-6}$ & $7.6 \times 10^{5}$ & $9.0 \times 10^{-6}$ & $4.3 \times 10^{-3}$ & $1.5 \times 10^{-3}$ & & $2.6 \times 10^{-2}$ & $4.1 \times 10^{-5}$ & $3.7 \times 10^{4}$ & $2.5 \times 10^{-4}$ & $5.2 \times 10^{-2}$ & $1.1 \times 10^{0}$ & $3.2 \times 10^{-2}$ & $1.2 \times 10^{0}$ \\
\hline 1976 & $4.0 \times 10^{-5}$ & $1.6 \times 10^{-5}$ & $1.9 \times 10^{-5}$ & & $2.0 \times 10^{3}$ & $2.8 \times 10^{-5}$ & & $1.3 \times 10^{-2}$ & $2.0 \times 10^{-5}$ & $7.8 \times 10^{-6}$ & $6.5 \times 10^{-5}$ & $4.7 \times 10^{-2}$ & $1.4 \times 10^{0}$ & $3.0 \times 10^{-2}$ & $1.5 \times 10^{0}$ \\
\hline 1977 & $1.6 \times 10^{-5}$ & $3.1 \times 10^{-5}$ & $1.1 \times 10^{-3}$ & & $3.6 \times 10^{-3}$ & $1.5 \times 10^{-4}$ & & $1.5 \times 10^{-2}$ & $1.2 \times 10^{-4}$ & $8.7 \times 10^{-6}$ & $1.1 \times 10^{-3}$ & $3.8 \times 10^{-2}$ & $1.1 \times 10^{0}$ & $7.7 \times 10^{-3}$ & $1.2 \times 10^{0}$ \\
\hline 1978 & & $2.6 \times 10^{-6}$ & $5.5 \times 10^{-6}$ & & $2.0 \times 10^{-3}$ & $1.5 \times 10^{-5}$ & & $1.4 \times 10^{-2}$ & $6.1 \times 10^{-7}$ & & $1.0 \times 10^{-5}$ & $3.5 \times 10^{-2}$ & $1.3 \times 10^{\circ}$ & $6.8 \times 10^{-3}$ & $1.4 \times 10^{0}$ \\
\hline 1979 & $8.9 \times 10^{6}$ & $2.3 \times 10^{-5}$ & $3.8 \times 10^{3}$ & & $1.3 \times 10^{-3}$ & & & $1.4 \times 10^{-2}$ & & & & $2.3 \times 10^{-2}$ & $6.6 \times 10^{-1}$ & $5.1 \times 10^{-3}$ & $7.1 \times 10^{-1}$ \\
\hline 1980 & & & $1.5 \times 10^{-5}$ & & $1.0 \times 10^{-3}$ & & & $9.0 \times 10^{-3}$ & & & & $1.9 \times 10^{-2}$ & $5.6 \times 10^{-1}$ & $1.5 \times 10^{-2}$ & $6.0 \times 10^{-1}$ \\
\hline 1981 & & & $4.5 \times 10^{-6}$ & & $2.8 \times 10^{-3}$ & & & $2.4 \times 10^{-2}$ & & & & $3.4 \times 10^{-2}$ & $1.1 \times 10^{0}$ & $2.8 \times 10^{-2}$ & $1.2 \times 10^{0}$ \\
\hline 1982 & & & $1.7 \times 10^{-6}$ & & $1.7 \times 10^{-3}$ & & & $2.5 \times 10^{-2}$ & & & & $2.5 \times 10^{-2}$ & $1.2 \times 10^{0}$ & $5.8 \times 10^{-3}$ & $1.3 \times 10^{0}$ \\
\hline 1983 & & & $2.1 \times 10^{-5}$ & & $1.1 \times 10^{-3}$ & & & $1.2 \times 10^{-2}$ & & & & $1.8 \times 10^{-2}$ & $9.0 \times 10^{-1}$ & $1.6 \times 10^{-3}$ & $9.3 \times 10^{-1}$ \\
\hline 1984 & & & $2.9 \times 10^{-6}$ & & $1.5 \times 10^{-3}$ & & & $4.9 \times 10^{-2}$ & & & & $1.7 \times 10^{-2}$ & $7.4 \times 10^{-1}$ & $7.9 \times 10^{-4}$ & $8.1 \times 10^{-1}$ \\
\hline 1985 & & & & & $1.2 \times 10^{-3}$ & & & $2.1 \times 10^{-2}$ & & & & $1.8 \times 10^{-2}$ & $1.1 \times 10^{0}$ & $2.2 \times 10^{-4}$ & $1.1 \times 10^{0}$ \\
\hline 1986 & & & & & $1.5 \times 10^{-3}$ & & & $3.0 \times 10^{-2}$ & & & & $3.0 \times 10^{-2}$ & $1.4 \times 10^{0}$ & $8.2 \times 10^{-3}$ & $1.5 \times 10^{0}$ \\
\hline 1987 & & & & & $3.3 \times 10^{-3}$ & & & $3.9 \times 10^{-2}$ & & & & $1.8 \times 10^{-2}$ & $7.0 \times 10^{-1}$ & $6.1 \times 10^{-4}$ & $7.6 \times 10^{-1}$ \\
\hline 1988 & & & & & $8.5 \times 10^{-3}$ & & & $2.1 \times 10^{-2}$ & & & & $2.3 \times 10^{-2}$ & $1.0 \times 10^{0}$ & $1.1 \times 10^{-3}$ & $1.1 \times 10^{0}$ \\
\hline 1989 & & & & & $2.6 \times 10^{-3}$ & & & $4.4 \times 10^{-2}$ & $1.3 \times 10^{-7}$ & $7.1 \times 10^{-5}$ & $6.2 \times 10^{-7}$ & $2.4 \times 10^{-2}$ & $7.8 \times 10^{-1}$ & $6.8 \times 10^{-5}$ & $8.5 \times 10^{-1}$ \\
\hline 1990 & $7.8 \times 10^{-6}$ & & & & $4.1 \times 10^{4}$ & & & $4.7 \times 10^{-2}$ & & & & $2.7 \times 10^{-2}$ & $4.9 \times 10^{-1}$ & $1.5 \times 10^{.4}$ & $5.6 \times 10^{-1}$ \\
\hline 1991 & & & & & $1.8 \times 10^{-4}$ & & & $3.4 \times 10^{-2}$ & & & & $3.0 \times 10^{-2}$ & $6.7 \times 10^{-1}$ & $2.8 \times 10^{-4}$ & $7.3 \times 10^{-1}$ \\
\hline 1992 & & & & & $1.1 \times 10^{-3}$ & & & $4.4 \times 10^{-2}$ & & & & $6.5 \times 10^{-2}$ & $5.5 \times 10^{-1}$ & $2.7 \times 10^{-4}$ & $6.6 \times 10^{-1}$ \\
\hline 1993 & & & & & $1.9 \times 10^{-3}$ & & & $1.8 \times 10^{-2}$ & & & & $2.6 \times 10^{-2}$ & $3.4 \times 10^{-1}$ & $1.0 \times 10^{-6}$ & $3.9 \times 10^{-1}$ \\
\hline 1994 & & & & & $5.0 \times 10^{3}$ & & & $3.1 \times 10^{-2}$ & & & & $2.6 \times 10^{-2}$ & $3.5 \times 10^{-1}$ & $1.1 \times 10^{-6}$ & $4.1 \times 10^{-1}$ \\
\hline 1995 & & & $2.8 \times 10^{-5}$ & & $4.8 \times 10^{-3}$ & & $3.9 \times 10^{-6}$ & $3.3 \times 10^{-2}$ & & & & $2.5 \times 10^{-2}$ & $3.3 \times 10^{-1}$ & $3.5 \times 10^{-5}$ & $3.9 \times 10^{-1}$ \\
\hline 1996 & & & & & $4.9 \times 10^{-3}$ & & $1.7 \times 10^{.5}$ & $8.0 \times 10^{-2}$ & & & & $2.1 \times 10^{-2}$ & $3.5 \times 10^{-1}$ & $2.6 \times 10^{-3}$ & $4.6 \times 10^{-1}$ \\
\hline Total & $9.5 \times 10^{-2}$ & $2.0 \times 10^{-1}$ & $7.5 \times 10^{-1}$ & $8.3 \times 10^{-1}$ & $4.6 \times 10^{0}$ & $5.3 \times 10^{0}$ & $4.0 \times 10^{-1}$ & $1.6 \times 10^{0}$ & $1.7 \times 10^{-1}$ & $5.2 \times 10^{-1}$ & $2.7 \times 10^{0}$ & $8.6 \times 10^{0}$ & $4.7 \times 10^{1}$ & $2.6 \times 10^{0}$ & $7.5 \times 10^{1}$ \\
\hline
\end{tabular}




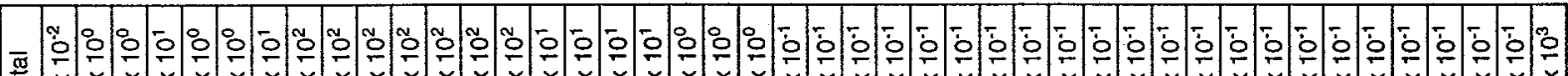

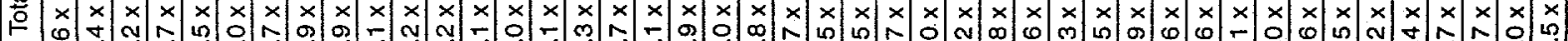
ம்

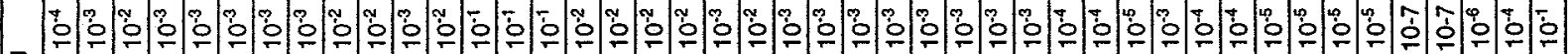

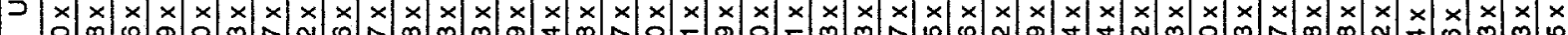

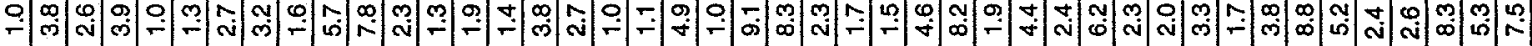

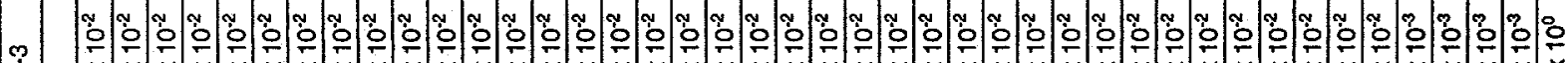

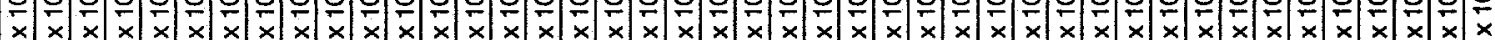

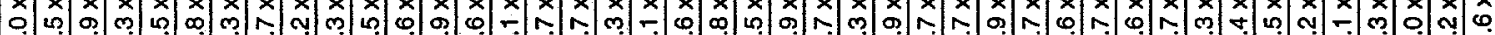

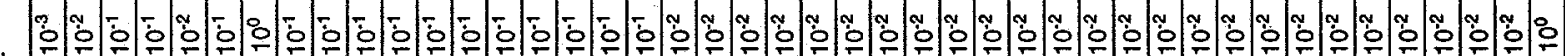

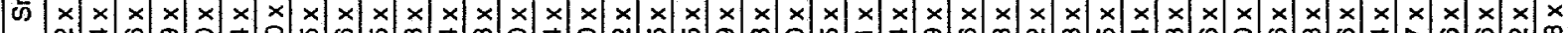

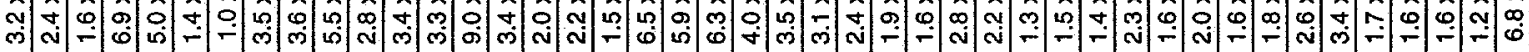

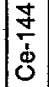

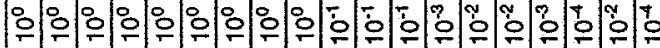

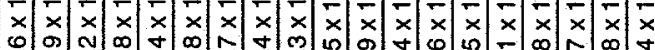

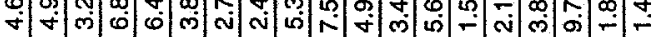

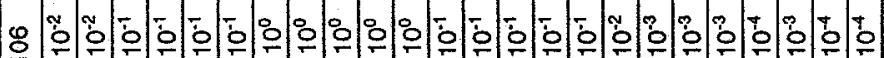

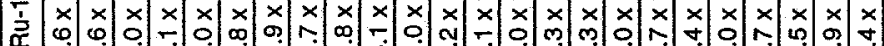
व

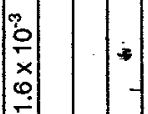

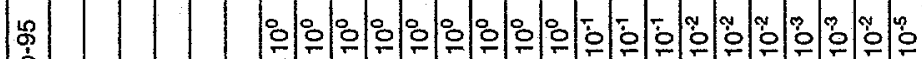

$\sum_{\hat{N}}$

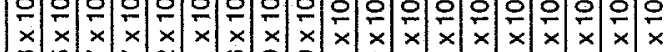

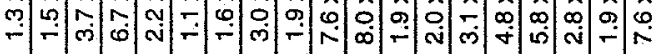

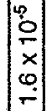

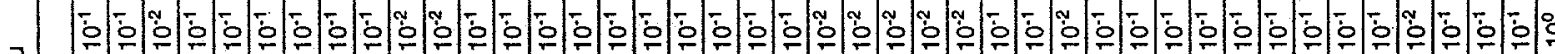

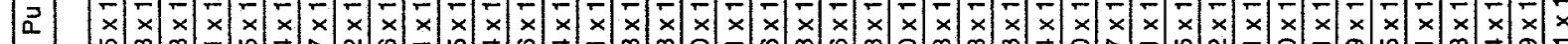

\begin{tabular}{|c|c|c|c|c|c|c|c|c|c|c|c|c|c|c|c|c|c|c|c|c|c|c|c|c|c|c|c|}
\hline & {$\left[\begin{array}{l}1 \\
0 \\
\infty\end{array}\right]$} & & $\begin{array}{c}3 \\
0 \\
\infty\end{array}=$ & {$[0$} & 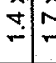 & $\hat{\square}$ & $\stackrel{0}{\stackrel{0}{\infty}}$ & $\left.\mid \begin{array}{l}0 \\
\infty\end{array}\right]$ & $\stackrel{0}{\circ}$ & $\mid \begin{array}{l}f \\
:\end{array}$ & $=\left[\begin{array}{l}0 \\
0 \\
\sim\end{array}\right.$ & & $\frac{\sigma}{m}$ & : & \begin{tabular}{|c|}
0 \\
0 \\
0
\end{tabular} & 象 & $\hat{m}$ & $\begin{array}{l}\hat{q} \\
\dot{q} \\
\dot{*}\end{array}$ & $\therefore 0$ & $\begin{array}{c}0 \\
0\end{array}$ & : & $=0$ & $\hat{v}$ & $\stackrel{0}{-0}$ & : & $\vec{\nabla}: 0$ & $\mathbf{N} \mid \infty$ \\
\hline 8 & & & & & & & & & & $\begin{array}{l}5 \\
0 \\
x \\
1 \\
0 \\
0\end{array}$ & & & $\left|\begin{array}{l}9 \\
0 \\
0 \\
0 \\
0 \\
\end{array}\right|$ & & & & & & & & & & & & & 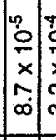 & 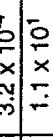 \\
\hline 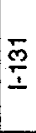 & & & $\begin{array}{l}8 \\
\stackrel{0}{0} \\
x \\
0 \\
\dot{0}\end{array}$ & & 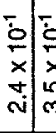 & 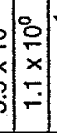 & 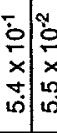 & $\left|\begin{array}{c}0 \\
0 \\
x \\
0 \\
6 \\
1\end{array}\right|$ & 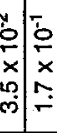 & 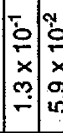 & 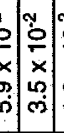 & & $\mid \begin{array}{l}0 \\
0 \\
0 \\
0 \\
0\end{array}$ & \begin{tabular}{l|l}
0 & 0 \\
0 & 0 \\
0 \\
0 \\
0 \\
0 \\
0 \\
0
\end{tabular} & $\left.\mid \begin{array}{l}0 \\
0 \\
0 \\
0 \\
0 \\
\hdashline\end{array}\right]$ & 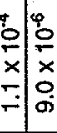 & & & & & & & & & & & $\mid \begin{array}{l}0 \\
0 \\
x \\
\infty \\
\infty \\
j\end{array}$ \\
\hline$\hat{\mathrm{m}}$ & $\mid \begin{array}{l}\overline{0} \\
\dot{0} \\
x \\
0 \\
\dot{s}\end{array}$ & $\begin{array}{l}0 \\
\dot{0} \\
0 \\
0 \\
0\end{array}$ & 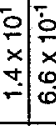 & $\mid$\begin{tabular}{ll}
0 \\
0 \\
$x$ \\
$x$ \\
0 \\
0 \\
\hdashline
\end{tabular} & $\mid \begin{array}{l}0 \\
0 \\
x \\
x \\
0 \\
0 \\
0\end{array}$ & $\mid \begin{array}{l}0 \\
0 \\
x \\
x \\
0 \\
\infty \\
0\end{array}$ & $\begin{array}{l}0 \\
x \\
x \\
\end{array}$ & $\mid \begin{array}{l}0 \\
0 \\
x \\
0 \\
0\end{array}$ & 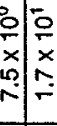 & {$\left[\begin{array}{l}0 \\
0 \\
x \\
0 \\
0 \\
0\end{array}\right.$} & 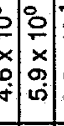 & & $\left(\begin{array}{c}0 \\
0 \\
x \\
0 \\
0\end{array}\right.$ & 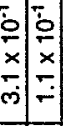 & & & & & & & & & & & & $\mid \begin{array}{l}\dot{0} \\
\dot{x} \\
0 \\
-\end{array}$ & 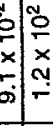 \\
\hline ఝ & & & & & 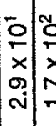 & $=\mid \begin{array}{c}0 \\
0 \\
x \\
n \\
j\end{array}$ & & $\mid$\begin{tabular}{l}
0 \\
\hdashline \\
$x$ \\
0 \\
0
\end{tabular} & 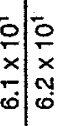 & 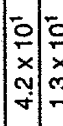 & 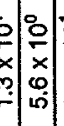 & $\mid$\begin{tabular}{|c|}
0 \\
$\frac{0}{x}$ \\
$x$ \\
\\
\end{tabular} & & 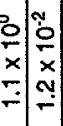 & & & & & & & & & & & & & $\stackrel{0}{\frac{0}{x}} \stackrel{0}{\underline{x}}$ \\
\hline ष & 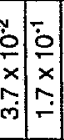 & & 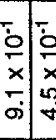 & 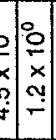 & 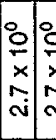 & 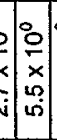 & 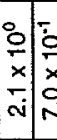 & \begin{tabular}{l} 
\\
0 \\
\hdashline \\
0 \\
0 \\
0
\end{tabular} & 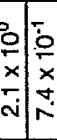 & 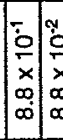 & 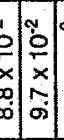 & 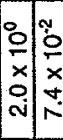 & $\left|\begin{array}{c|c}0 \\
0 \\
0 \\
\hdashline \\
x \\
0 \\
0 \\
0\end{array}\right|$ & 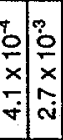 & \begin{tabular}{|l|} 
\\
0 \\
0 \\
$x$ \\
0 \\
0 \\
0
\end{tabular} & \begin{tabular}{l|l}
9 \\
0 \\
0
\end{tabular} & \begin{tabular}{|c|} 
\\
\\
\hdashline \\
\\
\end{tabular} & & 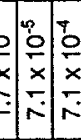 & 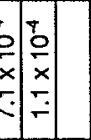 & & & & & & $\left|\begin{array}{l}0 \\
0 \\
x \\
0 \\
\infty\end{array}\right|$ & $\mid$\begin{tabular}{l}
$\overline{0}$ \\
\hdashline \\
$x$ \\
$\hat{\alpha}$ \\
\end{tabular} \\
\hline$\frac{\square}{0}$ & & & & & 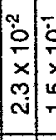 & 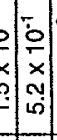 & 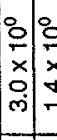 & $\mid \begin{array}{c}0 \\
0 \\
x \\
x \\
0 \\
2\end{array}$ & 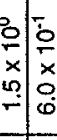 & 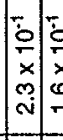 & 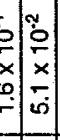 & 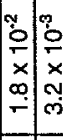 & 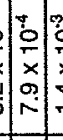 & 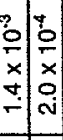 & 0 & 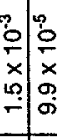 & $\begin{array}{l}0 \\
0 \\
\frac{0}{2} \\
\\
\end{array}$ & & & & & & & & & & $\mid \begin{array}{l}0 \\
0 \\
x \\
x \\
0 \\
0\end{array}$ \\
\hline $\mid \begin{array}{l}\tilde{m} \\
0 \\
0\end{array}$ & & & & & & & ic & $\mid$ & 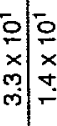 & 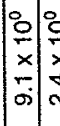 & 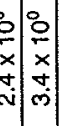 & 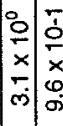 & 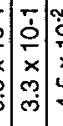 & 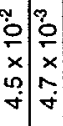 & $\begin{array}{l}x \\
0 \\
0 \\
x \\
y \\
\dot{y}\end{array} \mid$ & $\begin{array}{l}\underline{y} \\
\dot{x} \\
\text { à } \\
\text { s. }\end{array}$ & \begin{tabular}{l}
0 \\
0 \\
0 \\
\hdashline \\
$y$
\end{tabular} & & & & & & $\left|\begin{array}{l}0 \\
0 \\
x \\
n \\
n \\
x\end{array}\right|$ & & & & $\frac{\tilde{o}}{\frac{0}{x}}$ \\
\hline 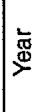 & \begin{tabular}{|l|l}
0 \\
0
\end{tabular} & 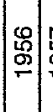 & 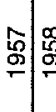 & 雚总品 & 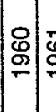 & ఏ气 & 8 & ஜ & 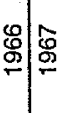 & $\mid \begin{array}{l}0 \\
: 8\end{array}$ & : & 장 & 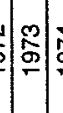 & 蒿器 & హ. & 송 & 足 & $\bar{x}$ & 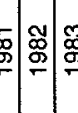 & 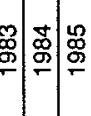 & 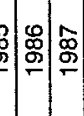 & $\stackrel{\infty}{\infty}$ & गֶ: & চু & 总 & 造 & 吕 \\
\hline
\end{tabular}




\begin{tabular}{|c|c|c|c|c|c|c|c|c|c|c|c|c|c|c|c|c|}
\hline Year & $p-32$ & Cr-51 & $\mathrm{C} 0-60$ & $Z n-65$ & Cs-137 & $1-131$ & $\mathrm{Cm}-244$ & $\mathrm{Pu}$ & $\mathrm{Zr}, \mathrm{Nb}-95$ & Ru-106 & $\overline{\mathrm{Ce}} \cdot 144$ & $\mathrm{Sr}$ & Tc-99 & $\mathrm{H} \cdot 3$ & U & Total \\
\hline 1954 & & & $3.8 \times 10^{-2}$ & & & & & & & $1.7 \times 10^{-2}$ & & $7.3 \times 10^{-3}$ & & & $4.7 \times 10^{-4}$ & $6.3 \times 10^{-2}$ \\
\hline 1955 & & & $1.8 \times 10^{1}$ & & $2.7 \times 10^{-1}$ & & & $1.0 \times 10^{0}$ & & $7.9 \times 10^{-2}$ & & $5.3 \times 10^{-2}$ & & $3.1 \times 10^{-1}$ & $1.7 \times 10^{-2}$ & $1.9 \times 10^{0}$ \\
\hline 1956 & & & $4.5 \times 10^{-1}$ & & $6.8 \times 10^{-1}$ & & & $8.8 \times 10^{-1}$ & & $2.0 \times 10^{-1}$ & & $3.6 \times 10^{-1}$ & & $4.8 \times 10^{-1}$ & $1.2 \times 10^{-1}$ & $3.2 \times 10^{0}$ \\
\hline 1957 & & & $9.4 \times 10^{-1}$ & & $1.4 \times 10^{1}$ & $2.4 \times 10^{0}$ & & $6.3 \times 10^{-2}$ & & $7.4 \times 10^{-1}$ & & $1.6 \times 10^{0}$ & & $5.8 \times 10^{-1}$ & $1.7 \times 10^{-2}$ & $2.0 \times 10^{1}$ \\
\hline 1958 & & & $4.6 \times 10^{-1}$ & & $6.9 \times 10^{-1}$ & & & $1.3 \times 10^{-1}$ & & $2.1 \times 10^{-1}$ & & $1.1 \times 10^{-1}$ & & \begin{tabular}{|l}
$.1 \times 10^{-1}$ \\
\end{tabular} & $4.7 \times 10^{-3}$ & $2.3 \times 10^{0}$ \\
\hline 1959 & & & $1.3 \times 10^{0}$ & & $1.9 \times 10^{\circ}$ & & & $1.8 \times 10^{-1}$ & & $6.0 \times 10^{-1}$ & & $3.1 \times 10^{-1}$ & & $1.4 \times 10^{0}$ & $5.7 \times 10^{3}$ & $5.7 \times 10^{0}$ \\
\hline 1960 & & $2.3 \times 10^{-2}$ & $2.8 \times 10^{0}$ & $2.9 \times 10^{1}$ & $6.3 \times 10^{0}$ & $5.7 \times 10^{-1}$ & & $1.7 \times 10^{-1}$ & $1.3 \times 10^{0}$ & $1.9 \times 10^{0}$ & $4.9 \times 10^{0}$ & $2.3 \times 10^{0}$ & & $1.2 \times 10^{0}$ & $1.2 \times 10^{-2}$ & $5.0 \times 10^{1}$ \\
\hline 1961 & & $1.5 \times 10^{-1}$ & $2.8 \times 10^{0}$ & $1.7 \times 10^{2}$ & $4.1 \times 10^{\circ}$ & $8.3 \times 10^{-1}$ & & $2.0 \times 10^{-1}$ & $1.5 \times 10^{0}$ & $1.8 \times 10^{0}$ & $5.2 \times 10^{0}$ & $7.9 \times 10^{-1}$ & & $1.6 \times 10^{0}$ & $1.4 \times 10^{2}$ & $1.9 \times 10^{2}$ \\
\hline 1962 & & $5.3 \times 10^{-1}$ & $5.7 \times 10^{0}$ & $2.7 \times 10^{2}$ & $8.3 \times 10^{0}$ & $2.6 \times 10^{0}$ & & $1.4 \times 10^{-1}$ & $3.8 \times 10^{0}$ & $1.8 \times 10^{0}$ & $3.4 \times 10^{0}$ & $8.2 \times 10^{-1}$ & & $1.5 \times 10^{0}$ & $7.2 \times 10^{-2}$ & $3.0 \times 10^{2}$ \\
\hline 1963 & & $3.1 \times 10^{0}$ & $2.1 \times 10^{0}$ & $2.7 \times 10^{2}$ & $2.1 \times 10^{1}$ & $1.3 \times 10^{0}$ & & $1.9 \times 10^{-1}$ & $6.8 \times 10^{0}$ & $3.2 \times 10^{0}$ & $7.3 \times 10^{0}$ & $1.2 \times 10^{0}$ & & $2.2 \times 10^{0}$ & $2.6 \times 10^{-1}$ & $3.2 \times 10^{2}$ \\
\hline 1964 & $5.4 \times 10^{0}$ & $1.4 \times 10^{0}$ & $7.2 \times 10^{-1}$ & $8.7 \times 10^{1}$ & $1.3 \times 10^{1}$ & $1.3 \times 10^{-1}$ & & $9.6 \times 10^{-2}$ & $2.2 \times 10^{0}$ & $1.1 \times 10^{0}$ & $6.8 \times 10^{0}$ & $6.3 \times 10^{-1}$ & & $1.3 \times 10^{0}$ & $3.5 \times 10^{-2}$ & $1.2 \times 10^{2}$ \\
\hline 1965 & $3.8 \times 10^{1}$ & $1.7 \times 10^{0}$ & $4.2 \times 10^{0}$ & $6.3 \times 10^{1}$ & $6.6 \times 10^{\circ}$ & $2.8 \times 10^{-1}$ & & $1.5 \times 10^{-1}$ & $1.1 \times 10^{0}$ & $9.1 \times 10^{-1}$ & $4.5 \times 10^{0}$ & $1.6 \times 10^{\circ}$ & & $7.4 \times 10^{0}$ & $2.5 \times 10^{-1}$ & $1.3 \times 10^{2}$ \\
\hline 1966 & $3.3 \times 10^{1}$ & $1.6 \times 10^{0}$ & $2.3 \times 10^{0}$ & $6.1 \times 10^{1}$ & $8.4 \times 10^{0}$ & $2.1 \times 10^{-1}$ & & $2.3 \times 10^{-1}$ & $1.7 \times 10^{0}$ & $3.5 \times 10^{-1}$ & $3.3 \times 10^{0}$ & $1.8 \times 10^{0}$ & & $8.1 \times 10^{0}$ & $1.7 \times 10^{0}$ & $1.2 \times 10^{2}$ \\
\hline 1967 & $1.4 \times 10^{1}$ & $6.4 \times 10^{-1}$ & $8.0 \times 10^{-1}$ & $6.2 \times 10^{1}$ & $1.9 \times 10^{1}$ & $9.1 \times 10^{-1}$ & & $2.6 \times 10^{-1}$ & $3.1 \times 10^{0}$ & $5.5 \times 10^{-1}$ & $2.8 \times 10^{0}$ & $4.3 \times 10^{0}$ & & $6.2 \times 10^{0}$ & $2.3 \times 10^{0}$ & $1.2 \times 10^{2}$ \\
\hline 1968 & $9.1 \times 10^{0}$ & $2.5 \times 10^{-1}$ & $9.6 \times 10^{-1}$ & $4.2 \times 10^{1}$ & $1.2 \times 10^{1}$ & $7.5 \times 10^{-1}$ & & $2.3 \times 10^{-1}$ & $2.0 \times 10^{0}$ & $6.0 \times 10^{-1}$ & $6.5 \times 10^{0}$ & $1.8 \times 10^{0}$ & & $6.9 \times 10^{0}$ & $1.8 \times 10^{0}$ & $8.5 \times 10^{1}$ \\
\hline 1969 & $2.4 \times 10^{0}$ & $1.7 \times 10^{-1}$ & $9.6 \times 10^{-2}$ & $1.3 \times 10^{1}$ & $5.1 \times 10^{\circ}$ & $3.2 \times 10^{-1}$ & $9.4 \times 10^{-1}$ & $1.7 \times 10^{-1}$ & $7.7 \times 10^{-1}$ & $1.4 \times 10^{-1}$ & $9.0 \times 10^{-1}$ & $9.6 \times 10^{-1}$ & & $5.7 \times 10^{0}$ & $4.5 \times 10^{-1}$ & $3.1 \times 10^{1}$ \\
\hline 1970 & $3.4 \times 10^{\circ}$ & $5.3 \times 10^{-2}$ & $1.0 \times 10^{-1}$ & $5.6 \times 10^{0}$ & $6.4 \times 10^{\circ}$ & $1.5 \times 10^{-1}$ & & $4.0 \times 10^{-1}$ & $8.1 \times 10^{-1}$ & $2.2 \times 10^{-2}$ & $5.6 \times 10^{-1}$ & $8.3 \times 10^{-1}$ & & $3.2 \times 10^{0}$ & $2.4 \times 10^{-1}$ & $2.2 \times 10^{1}$ \\
\hline 1971 & $3.1 \times 10^{0}$ & $1.9 \times 10^{-2}$ & $2.1 \times 10^{0}$ & $1.5 \times 10^{1}$ & $7.0 \times 10^{-1}$ & $7.0 \times 10^{-2}$ & $1.0 \times 10^{1}$ & $6.0 \times 10^{-1}$ & $1.9 \times 10^{-1}$ & $9.4 \times 10^{-3}$ & $3.9 \times 10^{-1}$ & $5.3 \times 10^{-1}$ & & $2.3 \times 10^{0}$ & $8.5 \times 10^{-2}$ & $3.5 \times 10^{1}$ \\
\hline 1972 & $9.6 \times 10^{-1}$ & $3.4 \times 10^{-3}$ & $8.0 \times 10^{-2}$ & & $2.9 \times 10^{-1}$ & $5.2 \times 10^{-2}$ & $8.5 \times 10^{-1}$ & $1.1 \times 10^{0}$ & $2.1 \times 10^{-2}$ & $1.0 \times 10^{-2}$ & $6.6 \times 10^{-3}$ & $2.9 \times 10^{-1}$ & & $3.5 \times 10^{0}$ & $1.2 \times 10^{-1}$ & $7.3 \times 10^{0}$ \\
\hline 1973 & $3.3 \times 10^{-1}$ & $8.6 \times 10^{-4}$ & $9.6 \times 10^{-3}$ & & $1.6 \times 10^{-1}$ & $9.0 \times 10^{-3}$ & $8.9 \times 10^{-2}$ & $5.3 \times 10^{-1}$ & $3.1 \times 10^{-2}$ & $1.0 \times 10^{-2}$ & $1.9 \times 10^{-2}$ & $3.4 \times 10^{-1}$ & & $5.7 \times 10^{0}$ & $6.9 \times 10^{-2}$ & $7.3 \times 10^{0}$ \\
\hline 1974 & $4.6 \times 10^{-2}$ & $1.5 \times 10^{-3}$ & $4.5 \times 10^{-4}$ & $1.1 \times 10^{0}$ & $3.4 \times 10^{-1}$ & $8.9 \times 10^{-4}$ & & $2.5 \times 10^{-1}$ & $5.0 \times 10^{-2}$ & $1.1 \times 10^{-3}$ & $2.5 \times 10^{-2}$ & $3.1 \times 10^{-1}$ & & $5.3 \times 10^{0}$ & $1.2 \times 10^{-1}$ & $7.5 \times 10^{0}$ \\
\hline 1975 & $4.7 \times 10^{-3}$ & $2.1 \times 10^{-4}$ & $2.9 \times 10^{-3}$ & $1.2 \times 10^{-2}$ & $1.3 \times 10^{-1}$ & $5.5 \times 10^{-3}$ & & $2.1 \times 10^{-1}$ & $5.9 \times 10^{-3}$ & $1.1 \times 10^{-2}$ & $4.6 \times 10^{-3}$ & $2.0 \times 10^{-1}$ & & $3.5 \times 10^{\circ}$ & $1.1 \times 10^{-1}$ & $4.2 \times 10^{0}$ \\
\hline 1976 & $4.4 \times 10^{-2}$ & $7.1 \times 10^{-4}$ & $7.1 \times 10^{-4}$ & & $5.4 \times 10^{-2}$ & $9.1 \times 10^{-5}$ & & $1.0 \times 10^{-1}$ & $2.9 \times 10^{-3}$ & $2.1 \times 10^{-4}$ & $1.1 \times 10^{-3}$ & $1.6 \times 10^{-1}$ & & $3.6 \times 10^{0}$ & $8.8 \times 10^{-2}$ & $4.1 \times 10^{0}$ \\
\hline 1977 & $2.0 \times 10^{-2}$ & $1.6 \times 10^{-3}$ & $4.4 \times 10^{-2}$ & & $1.1 \times 10^{-1}$ & $6.5 \times 10^{-4}$ & & $1.3 \times 10^{-1}$ & $1.9 \times 10^{-2}$ & $2.7 \times 10^{-4}$ & $2.2 \times 10^{-2}$ & $1.7 \times 10^{-1}$ & & $4.0 \times 10^{0}$ & $3.0 \times 10^{-2}$ & $4.5 \times 10^{0}$ \\
\hline 1978 & & $1.1 \times 10^{-4}$ & $1.8 \times 10^{-4}$ & & $5.1 \times 10^{-2}$ & $4.7 \times 10^{-5}$ & & $9.5 \times 10^{-2}$ & $7.8 \times 10^{-5}$ & & $1.6 \times 10^{-4}$ & $1.1 \times 10^{-1}$ & & $3.3 \times 10^{\circ}$ & $2.0 \times 10^{-2}$ & $3.6 \times 10^{0}$ \\
\hline 1979 & $1.1 \times 10^{-2}$ & $1.1 \times 10^{-3}$ & $1.5 \times 10^{-1}$ & & $4.0 \times 10^{-2}$ & & & $1.1 \times 10^{-1}$ & & & & $8.1 \times 10^{-2}$ & & $1.8 \times 10^{0}$ & $1.5 \times 10^{-2}$ & $2.2 \times 10^{0}$ \\
\hline 1980 & & & $5.9 \times 10^{-4}$ & & $3.0 \times 10^{-2}$ & & & $7.4 \times 10^{-2}$ & & & & $7.1 \times 10^{-2}$ & & $1.6 \times 10^{0}$ & $5.0 \times 10^{-2}$ & $1.8 \times 10^{0}$ \\
\hline 1981 & & & $1.8 \times 10^{-4}$ & & $8.6 \times 10^{-2}$ & & & $2.1 \times 10^{-1}$ & & & & $1.4 \times 10^{-1}$ & & $3.8 \times 10^{\circ}$ & $1.1 \times 10^{-1}$ & $4.3 \times 10^{0}$ \\
\hline 1982 & & & $7.5 \times 10^{-5}$ & & $5.6 \times 10^{-2}$ & & & $2.1 \times 10^{-1}$ & & & & $9.0 \times 10^{-2}$ & & $3.5 \times 10^{\circ}$ & $1.8 \times 10^{-2}$ & $3.9 \times 10^{0}$ \\
\hline 1983 & & & $8.1 \times 10^{-4}$ & & $3.2 \times 10^{-2}$ & & & $1.2 \times 10^{-1}$ & & & & $9.8 \times 10^{-2}$ & & $4.4 \times 10^{0}$ & $8.5 \times 10^{-3}$ & $4.7 \times 10^{0}$ \\
\hline 1984 & & & $1.2 \times 10^{-4}$ & & $4.7 \times 10^{-2}$ & & & $4.2 \times 10^{-1}$ & & & & $7.0 \times 10^{-2}$ & & $2.4 \times 10^{0}$ & $2.8 \times 10^{-3}$ & $2.9 \times 10^{0}$ \\
\hline 1985 & & & & & $3.6 \times 10^{-2}$ & & & $2.0 \times 10^{-1}$ & & & & $9.2 \times 10^{-2}$ & & $4.6 \times 10^{\circ}$ & $1.0 \times 10^{-3}$ & $4.9 \times 10^{0}$ \\
\hline 1986 & & & & & $4.5 \times 10^{-2}$ & & & $2.7 \times 10^{-1}$ & & & & $1.4 \times 10^{-1}$ & & $5.8 \times 10^{\circ}$ & $3.6 \times 10^{-2}$ & $6.3 \times 10^{0}$ \\
\hline 1987 & & & & & $1.1 \times 10^{-1}$ & & & $3.9 \times 10^{-1}$ & & & & $9.3 \times 10^{-2}$ & & $3.0 \times 10^{0}$ & $2.8 \times 10^{-3}$ & $3.6 \times 10^{0}$ \\
\hline 1988 & & & & & $2.7 \times 10^{-1}$ & & & $2.0 \times 10^{-4}$ & & & & $1.2 \times 10^{-1}$ & & $4.5 \times 10^{0}$ & $4.9 \times 10^{-3}$ & $5.1 \times 10^{0}$ \\
\hline 1989 & & & & & $7.0 \times 10^{-2}$ & & & $3.9 \times 10^{-1}$ & $1.6 \times 10^{.5}$ & $1.9 \times 10^{-3}$ & $1.1 \times 10^{-5}$ & $1.2 \times 10^{-1}$ & & $3.5 \times 10^{0}$ & $3.2 \times 10^{-4}$ & $4.1 \times 10^{0}$ \\
\hline 1990 & $7.9 \times 10^{-3}$ & & & & $1.1 \times 10^{-2}$ & & & $3.9 \times 10^{-1}$ & & & & $1.2 \times 10^{-1}$ & & $1.8 \times 10^{0}$ & $6.1 \times 10^{-4}$ & $2.3 \times 10^{0}$ \\
\hline 1991 & & & & & $5.8 \times 10^{-3}$ & & & $3.2 \times 10^{-1}$ & & 1 & & $1.4 \times 10^{-1}$ & & $2.5 \times 10^{0}$ & $1.2 \times 10^{-3}$ & $3.0 \times 10^{0}$ \\
\hline 1992 & & & & & $2.3 \times 10^{-2}$ & & & $3.1 \times 10^{-1}$ & & & & $2.7 \times 10^{-1}$ & & $2.0 \times 10^{0}$ & $1.1 \times 10^{-3}$ & $2.6 \times 10^{0}$ \\
\hline 1993 & & & & & $4.5 \times 10^{-2}$ & & & $1.3 \times 10^{-1}$ & & & & $1.1 \times 10^{-1}$ & & $1.2 \times 10^{0}$ & $3.9 \times 10.6$ & $1.5 \times 10^{0}$ \\
\hline 1994 & & & & & $1.2 \times 10^{-1}$ & & & $2.4 \times 10^{-1}$ & & & & $1.1 \times 10^{-1}$ & & $1.3 \times 10^{0}$ & $4.6 \times 10-6$ & $1.8 \times 10^{0}$ \\
\hline 1995 & & & $9.4 \times 10^{-4}$ & & $1.2 \times 10^{-1}$ & & $1.0 \times 10^{-4}$ & $2.6 \times 10^{-1}$ & & & & $1.1 \times 10^{-1}$ & & $1.3 \times 10^{0}$ & $1.5 \times 10^{-4}$ & $1.8 \times 10^{0}$ \\
\hline 1996 & & & & & $1.1 \times 10^{-1}$ & & $3.9 \times 10^{-4}$ & $6.1 \times 10^{-1}$ & & & & $9.6 \times 10^{-2}$ & & $1.4 \times 10^{0}$ & $1.1 \times 10^{-2}$ & $2.2 \times 10^{\circ}$ \\
\hline Total & $1.1 \times 10^{2}$ & $9.6 \times 10^{0}$ & $2.8 \times 10^{1}$ & $1.1 \times 10^{3}$ & $1.3 \times 10^{2}$ & $1.1 \times 10^{1}$ & $1.2 \times 10^{1}$ & $1.2 \times 10^{1}$ & $2.5 \times 10^{7}$ & $1.4 \times 10^{1}$ & $4.7 \times 10^{1}$ & $2.4 \times 10^{1}$ & $2.4 \times 10^{-1}$ & $1.3 \times 10^{2}$ & $8.2 \times 10^{\circ}$ & $1.7 \times 10^{3}$ \\
\hline
\end{tabular}


Assessment of Radionuclides

in the Savannah River Site Environment-Summary (U)

WSRC-TR-98-00162

Table 4-37. Total Population Dose from Releases by Radionuclide (person-rem)

\begin{tabular}{|c|c|c|c|c|c|c|}
\hline \multirow[t]{2}{*}{ Radionuclide } & \multirow[t]{2}{*}{ Atmospheric Dose } & \multicolumn{3}{|c|}{ Dose from Liquid Releases } & \multirow[t]{2}{*}{ Total Liquid Dose } & \multirow[t]{2}{*}{ Total Dose } \\
\hline & & Beaufort-Jasper & Port Wentworth & $80-\mathrm{km}$ & & \\
\hline P-32 & & $1.9 \times 10^{-1}$ & $9.5 \times 10^{-2}$ & $1.1 \times 10^{2}$ & $1.1 \times 10^{2}$ & $1.1 \times 10^{2}$ \\
\hline $\mathrm{Cr}-51$ & & $2.0 \times 10^{-1}$ & $2.0 \times 10^{-1}$ & $9.2 \times 10^{0}$ & $9.6 \times 10^{0}$ & $9.6 \times 10^{0}$ \\
\hline Co-60 & $6.5 \times 10-1$ & $5.4 \times 10^{-1}$ & $7.5 \times 10^{-1}$ & $2.7 \times 10^{1}$ & $2.8 \times 10^{1}$ & $2.9 \times 10^{1}$ \\
\hline C-14 & $3.0 \times 10^{1}$ & & & & & $3.0 \times 10^{1}$ \\
\hline $\mathrm{Zn}-65$ & & $4.2 \times 10^{-1}$ & $8.3 \times 10^{-1}$ & $1.1 \times 10^{3}$ & $1.1 \times 10^{3}$ & $1.1 \times 10^{3}$ \\
\hline Cs-137 & $3.4 \times 10^{1}$ & $4.1 \times 10^{0}$ & $4.6 \times 10^{0}$ & $1.2 \times 10^{2}$ & $1.3 \times 10^{2}$ & $1.6 \times 10^{2}$ \\
\hline $1-129$ & $1.0 \times 10^{2}$ & & & & & $1.0 \times 10^{2}$ \\
\hline$|-13|$ & $8.3 \times 10^{2}$ & $1.5 \times 10^{0}$ & $5.3 \times 10^{0}$ & $3.8 \times 10^{0}$ & $1.1 \times 10^{1}$ & $8.4 \times 10^{2}$ \\
\hline Am-241 & $1.2 \times 10^{0}$ & & & & & $1.2 \times 10^{0}$ \\
\hline $\mathrm{Cm}-244$ & $8.9 \times 10^{0}$ & $4.8 \times 10^{-1}$ & $4.0 \times 10^{-1}$ & $1.1 \times 10^{1}$ & $1.2 \times 10^{1}$ & $2.1 \times 10^{1}$ \\
\hline $\mathrm{Ar}-41$ & $1.9 \times 10^{2}$ & & & & & $1.9 \times 10^{2}$ \\
\hline $\mathrm{Pu}$ & $7.1 \times 10^{2}$ & $2.6 \times 10^{0}$ & $1.6 \times 10^{\circ}$ & $8.1 \times 10^{\circ}$ & $1.2 \times 10^{1}$ & $7.2 \times 10^{2}$ \\
\hline $\mathrm{Zr}, \mathrm{Nb}-95$ & & $1.4 \times 10^{-1}$ & $1.7 \times 10^{-1}$ & $2.5 \times 10^{1}$ & $2.5 \times 10^{1}$ & $2.5 \times 10^{1}$ \\
\hline Ru-106 & $1.2 \times 10^{2}$ & $1.9 \times 10^{-1}$ & $5.2 \times 10^{-1}$ & $1.4 \times 10^{1}$ & $1.4 \times 10^{1}$ & $1.3 \times 10^{2}$ \\
\hline Ce-144 & & $2.2 \times 10^{0}$ & $2.7 \times 10^{0}$ & $4.2 \times 10^{1}$ & $4.7 \times 10^{1}$ & $4.7 \times 10^{1}$ \\
\hline $\mathrm{Sr}$ & $6.3 \times 10^{\circ}$ & $8.3 \times 10^{0}$ & $8.6 \times 10^{0}$ & $6.8 \times 10^{0}$ & $2.4 \times 10^{1}$ & $3.0 \times 10^{1}$ \\
\hline TC-99 & $6.5 \times 10^{\circ}$ & & & & $2.4 \times 10^{-2}$ & $6.5 \times 10^{0}$ \\
\hline $\mathrm{H}-3$ & $1.1 \times 10^{3}$ & $8.2 \times 10^{1}$ & $4.7 \times 10^{1}$ & $1.6 \times 10^{0}$ & $1.3 \times 10^{2}$ & $1.2 \times 10^{3}$ \\
\hline U & $3.2 \times 10^{1}$ & $4.9 \times 10^{0}$ & $2.6 \times 10^{0}$ & $7.5 \times 10^{-1}$ & $8.2 \times 10^{0}$ & $4.0 \times 10^{1}$ \\
\hline Total & $3.2 \times 10^{3}$ & $1.1 \times 10^{2}$ & $7.5 \times 10^{1}$ & $1.5 \times 10^{3}$ & $1.7 \times 10^{3}$ & $4.8 \times 10^{3}$ \\
\hline
\end{tabular}


Chapter 4. Releases and Doses of Individual Radionuclides

Table 4-38. Percent of Population Dose Contributed by Each Radionuclide

\begin{tabular}{|c|c|c|c|c|c|c|}
\hline Radionuclide & $\begin{array}{c}\text { Atmospheric } \\
\text { Dose } \\
\text { (per-rem) }\end{array}$ & $\begin{array}{c}\text { Percent of } \\
\text { Atmospheric } \\
\text { Dose }\end{array}$ & $\begin{array}{c}\text { Liquid Dose } \\
\text { (per-rem) }\end{array}$ & $\begin{array}{c}\text { Percent of } \\
\text { Liquid Dose }\end{array}$ & $\begin{array}{c}\text { Total Dose } \\
\text { (per-rem) }\end{array}$ & $\begin{array}{c}\text { Percent of Total } \\
\text { Dose }\end{array}$ \\
\hline P-32 & & $1.1 \times 10^{2}$ & 6.7 & $1.1 \times 10^{2}$ & 2.3 \\
\hline Cr-51 & & $9.6 \times 10^{0}$ & 0.6 & $9.6 \times 10^{0}$ & 0.2 \\
\hline Co-60 & $6.5 \times 10^{-1}$ & 0.0 & $2.8 \times 10^{1}$ & 1.7 & $2.9 \times 10^{1}$ & 0.6 \\
\hline $\mathrm{C}-14$ & $3.0 \times 10^{4}$ & 0.9 & & & $3.0 \times 10^{1}$ & 0.6 \\
\hline $\mathrm{Zn}-65$ & & & $1.1 \times 10^{3}$ & 66.0 & $1.1 \times 10^{3}$ & 23.0 \\
\hline $\mathrm{Cs}-137$ & $3.4 \times 10^{1}$ & 1.1 & $1.3 \times 10^{2}$ & 7.9 & $1.6 \times 10^{2}$ & 3.3 \\
\hline $\mathrm{I}-129$ & $1.0 \times 10^{2}$ & 3.2 & & & $1.0 \times 10^{2}$ & 2.1 \\
\hline $\mathrm{I}-131$ & $8.3 \times 10^{2}$ & 26.2 & $1.1 \times 10^{1}$ & 0.6 & $8.4 \times 10^{2}$ & 17.5 \\
\hline $\mathrm{Am}-241$ & $1.2 \times 10^{0}$ & 0.0 & & & $1.2 \times 10^{0}$ & 0.0 \\
\hline $\mathrm{Cm}-244$ & $8.9 \times 10^{0}$ & 0.3 & $1.2 \times 10^{1}$ & 0.7 & $2.1 \times 10^{1}$ & 0.4 \\
\hline $\mathrm{Ar}-41$ & $1.9 \times 10^{2}$ & 6.0 & & & $1.9 \times 10^{2}$ & 4.0 \\
\hline $\mathrm{Pu}$ & $7.1 \times 10^{2}$ & 22.4 & $1.2 \times 10^{1}$ & 0.7 & $7.2 \times 10^{2}$ & 15.0 \\
\hline $\mathrm{Zr}, \mathrm{Nb}-95$ & & & $2.5 \times 10^{1}$ & 1.5 & $2.5 \times 10^{1}$ & 0.5 \\
\hline $\mathrm{RL}-106$ & $1.2 \times 10^{2}$ & 3.7 & $1.4 \times 10^{1}$ & 0.9 & $1.3 \times 10^{2}$ & 2.7 \\
\hline $\mathrm{Ce}-144$ & & & $4.7 \times 10^{1}$ & 2.8 & $4.7 \times 10^{1}$ & 1.0 \\
\hline $\mathrm{Sr}$ & $6.3 \times 10^{0}$ & 0.2 & $2.4 \times 10^{1}$ & 1.5 & $3.0 \times 10^{1}$ & 0.6 \\
\hline $\mathrm{Tc}-99$ & $6.5 \times 10^{0}$ & 0.2 & $2.4 \times 10^{-1}$ & 0.0 & $6.7 \times 10^{0}$ & 0.1 \\
\hline $\mathrm{H}-3$ & $1.1 \times 10^{3}$ & 34.6 & $1.3 \times 10^{2}$ & 7.9 & $1.2 \times 10^{3}$ & 25.1 \\
\hline $\mathrm{U}$ & $3.2 \times 10^{1}$ & 1.0 & $8.2 \times 10^{0}$ & 0.5 & $4.0 \times 10^{1}$ & 0.8 \\
\hline Total & $3.2 \times 10^{3}$ & & $1.7 \times 10^{3}$ & & $4.8 \times 10^{3}$ & \\
\hline
\end{tabular}


Assessment of Radionuclides

in the Savannah River Site Environment-Summary (U)

WSRC-TR-98-00162

Table 4-39. Total Population Dose by Year (person-rem)

\begin{tabular}{|c|c|c|c|c|c|c|}
\hline \multirow[t]{2}{*}{ Year } & \multirow[t]{2}{*}{$\begin{array}{l}\text { Dose from } \\
\text { Atmospheric } \\
\text { Releases }\end{array}$} & \multicolumn{4}{|c|}{ Dose from Liquid Releases } & \multirow[t]{2}{*}{ Total } \\
\hline & & Beaufort-Jasper & Port Wentworth & 80-Kilometer & Total & \\
\hline 1954 & $1.5 \times 10^{0}$ & & $6.1 \times 10^{-3}$ & $5.6 \times 10^{-2}$ & $6.3 \times 10^{-2}$ & $1.6 \times 10^{0}$ \\
\hline 1955 & $5.8 \times 10^{2}$ & & $5.3 \times 10^{-1}$ & $1.4 \times 10^{0}$ & $1.9 \times 10^{0}$ & $5.8 \times 10^{2}$ \\
\hline 1956 & $5.7 \times 10^{2}$ & & $9.4 \times 10^{-1}$ & $2.2 \times 10^{0}$ & $3.2 \times 10^{0}$ & $5.7 \times 10^{2}$ \\
\hline 1957 & $1.8 \times 10^{2}$ & & $3.4 \times 10^{0}$ & $1.7 \times 10^{1}$ & $2.0 \times 10^{1}$ & $2.0 \times 10^{2}$ \\
\hline 1958 & $1.4 \times 10^{2}$ & & $8.1 \times 10^{-3}$ & $1.5 \times 10^{0}$ & $2.3 \times 10^{\circ}$ & $1.4 \times 10^{2}$ \\
\hline 1959 & $1.4 \times 10^{2}$ & & $1.6 \times 10^{0}$ & $4.0 \times 10^{0}$ & $5.7 \times 10^{0}$ & $1.5 \times 10^{2}$ \\
\hline 1960 & $8.6 \times 10^{1}$ & & $3.5 \times 10^{0}$ & $4.7 \times 10^{1}$ & $5.0 \times 10^{1}$ & $1.4 \times 10^{2}$ \\
\hline 1961 & $1.2 \times 10^{2}$ & & $3.3 \times 10^{0}$ & $1.9 \times 10^{2}$ & $1.9 \times 10^{2}$ & $3.1 \times 10^{2}$ \\
\hline 1962 & $8.1 \times 10^{1}$ & & $4.4 \times 10^{0}$ & $2.9 \times 10^{2}$ & $3.0 \times 10^{2}$ & $3.8 \times 10^{2}$ \\
\hline 1963 & $7.9 \times 10^{1}$ & & $5.6 \times 10^{\circ}$ & $3.1 \times 10^{2}$ & $3.2 \times 10^{2}$ & $3.9 \times 10^{2}$ \\
\hline 1964 & $9.9 \times 10^{1}$ & & $2.8 \times 10^{\circ}$ & $1.2 \times 10^{2}$ & $1.2 \times 10^{2}$ & $2.2 \times 10^{2}$ \\
\hline 1965 & $7.1 \times 10^{1}$ & $7.3 \times 10^{0}$ & $3.8 \times 10^{\circ}$ & $1.2 \times 10^{2}$ & $1.3 \times 10^{2}$ & $2.0 \times 10^{2}$ \\
\hline 1966 & $6.5 \times 10^{1}$ & $9.8 \times 10^{0}$ & $3.9 \times 10^{\circ}$ & $1.1 \times 10^{2}$ & $1.2 \times 10^{2}$ & $1.9 \times 10^{2}$ \\
\hline 1967 & $5.9 \times 10^{1}$ & $1.0 \times 10^{1}$ & $5.0 \times 10^{\circ}$ & $1.0 \times 10^{2}$ & $1.2 \times 10^{2}$ & $1.7 \times 10^{2}$ \\
\hline 1968 & $7.4 \times 10^{1}$ & $9.5 \times 10^{0}$ & $4.0 \times 10^{\circ}$ & $7.1 \times 10^{1}$ & $8.5 \times 10^{1}$ & $1.6 \times 10^{2}$ \\
\hline 1969 & $1.7 \times 10^{2}$ & $5.4 \times 10^{\circ}$ & $2.6 \times 10^{0}$ & $2.3 \times 10^{1}$ & $3.1 \times 10^{1}$ & $2.0 \times 10^{2}$ \\
\hline 1970 & $5.1 \times 10^{1}$ & $2.8 \times 10^{\circ}$ & $2.2 \times 10^{0}$ & $1.7 \times 10^{1}$ & $2.2 \times 10^{1}$ & $7.3 \times 10^{1}$ \\
\hline 1971 & $5.6 \times 10^{1}$ & $2.0 \times 10^{0}$ & $1.9 \times 10^{0}$ & $3.1 \times 10^{1}$ & $3.5 \times 10^{1}$ & $9.1 \times 10^{1}$ \\
\hline 1972 & $5.6 \times 10^{1}$ & $2.8 \times 10^{\circ}$ & $1.6 \times 10^{0}$ & $2.9 \times 10^{0}$ & $7.3 \times 10^{0}$ & $6.3 \times 10^{1}$ \\
\hline 1973 & $4.5 \times 10^{1}$ & $4.5 \times 10^{0}$ & $1.9 \times 10^{0}$ & $1.0 \times 10^{0}$ & $7.3 \times 10^{0}$ & $5.2 \times 10^{1}$ \\
\hline 1974 & $5.3 \times 10^{1}$ & $3.7 \times 10^{0}$ & $2.0 \times 10^{0}$ & $1.8 \times 10^{0}$ & $7.6 \times 10^{0}$ & $6.1 \times 10^{1}$ \\
\hline 1975 & $3.0 \times 10^{1}$ & $2.5 \times 10^{0}$ & $1.2 \times 10^{0}$ & $3.7 \times 10^{-1}$ & $4.2 \times 10^{0}$ & $3.4 \times 10^{1}$ \\
\hline 1976 & $2.4 \times 10^{1}$ & $2.4 \times 10^{0}$ & $1.5 \times 10^{\circ}$ & $2.5 \times 10^{-1}$ & $4.1 \times 10^{\circ}$ & $2.8 \times 10^{1}$ \\
\hline 1977 & $2.4 \times 10^{1}$ & $3.1 \times 10^{0}$ & $1.2 \times 10^{\circ}$ & $3.5 \times 10^{-1}$ & $4.6 \times 10^{0}$ & $2.9 \times 10^{1}$ \\
\hline 1978 & $5.0 \times 10^{1}$ & $2.1 \times 10^{\circ}$ & $1.4 \times 10^{0}$ & $1.8 \times 10^{-1}$ & $3.6 \times 10^{0}$ & $5.4 \times 10^{1}$ \\
\hline 1979 & $2.0 \times 10^{1}$ & $1.2 \times 10^{0}$ & $7.4 \times 10^{-1}$ & $3.3 \times 10^{-1}$ & $2.3 \times 10^{0}$ & $2.2 \times 10^{1}$ \\
\hline 1980 & $2.2 \times 10^{1}$ & $1.2 \times 10^{0}$ & $6.2 \times 10^{-1}$ & $1.3 \times 10^{-1}$ & $1.9 \times 10^{0}$ & $2.4 \times 10^{1}$ \\
\hline 1981 & $2.5 \times 10^{1}$ & $2.9 \times 10^{0}$ & $1.2 \times 10^{0}$ & $3.1 \times 10^{-1}$ & $4.5 \times 10^{0}$ & $2.9 \times 10^{1}$ \\
\hline 1982 & $2.5 \times 10^{1}$ & $2.3 \times 10^{0}$ & $1.3 \times 10^{0}$ & $2.7 \times 10^{-1}$ & $3.9 \times 10^{0}$ & $2.9 \times 10^{1}$ \\
\hline 1983 & $3.3 \times 10^{1}$ & $3.6 \times 10^{0}$ & $9.3 \times 10^{-1}$ & $1.3 \times 10^{-1}$ & $4.7 \times 10^{0}$ & $3.8 \times 10^{1}$ \\
\hline 1984 & $3.9 \times 10^{1}$ & $1.8 \times 10^{\circ}$ & $8.1 \times 10^{-1}$ & $3.6 \times 10^{-1}$ & $3.0 \times 10^{\circ}$ & $4.2 \times 10^{1}$ \\
\hline 1985 & $2.7 \times 10^{1}$ & $3.7 \times 10^{0}$ & $1.2 \times 10^{\circ}$ & $2.0 \times 10^{-1}$ & $5.0 \times 10^{0}$ & $3.2 \times 10^{1}$ \\
\hline 1986 & $1.8 \times 10^{1}$ & $4.6 \times 10^{\circ}$ & $1.5 \times 10^{0}$ & $2.7 \times 10^{-1}$ & $6.3 \times 10^{\circ}$ & $2.4 \times 10^{1}$ \\
\hline 1987 & $2.8 \times 10^{1}$ & $2.4 \times 10^{\circ}$ & $7.7 \times 10^{-1}$ & $3.6 \times 10^{-1}$ & $3.6 \times 10^{\circ}$ & $3.2 \times 10^{1}$ \\
\hline 1988 & $1.6 \times 10^{1}$ & $3.6 \times 10^{0}$ & $1.1 \times 10^{\circ}$ & $4.1 \times 10^{-1}$ & $5.1 \times 10^{0}$ & $2.1 \times 10^{1}$ \\
\hline 1989 & $1.2 \times 10^{1}$ & $2.9 \times 10^{0}$ & $8.5 \times 10^{-1}$ & $3.0 \times 10^{-1}$ & $4.1 \times 10^{\circ}$ & $1.6 \times 10^{1}$ \\
\hline 1990 & $9.2 \times 10^{0}$ & $1.5 \times 10^{0}$ & $5.6 \times 10^{-1}$ & $2.6 \times 10^{-1}$ & $2.3 \times 10^{0}$ & $1.2 \times 10^{1}$ \\
\hline 1991 & $6.8 \times 10^{0}$ & $2.0 \times 10^{0}$ & $7.3 \times 10^{-1}$ & $2.5 \times 10^{-1}$ & $3.0 \times 10^{0}$ & $9.8 \times 10^{0}$ \\
\hline 1992 & $5.0 \times 10^{0}$ & $1.8 \times 10^{0}$ & $6.6 \times 10^{-1}$ & $2.2 \times 10^{-1}$ & $2.6 \times 10^{0}$ & $7.7 \times 10^{\circ}$ \\
\hline 1993 & $6.8 \times 10^{0}$ & $9.6 \times 10^{-1}$ & $3.9 \times 10^{-1}$ & $1.4 \times 10^{-1}$ & $1.5 \times 10^{0}$ & $8.3 \times 10^{0}$ \\
\hline 1994 & $5.6 \times 10^{0}$ & $1.1 \times 10^{0}$ & $4.1 \times 10^{-1}$ & $2.7 \times 10^{-1}$ & $1.8 \times 10^{0}$ & $7.4 \times 10^{0}$ \\
\hline 1995 & $3.1 \times 10^{0}$ & $1.1 \times 10^{0}$ & $3.9 \times 10^{-1}$ & $2.7 \times 10^{-1}$ & $1.8 \times 10^{0}$ & $4.9 \times 10^{0}$ \\
\hline 1996 & $2.6 \times 10^{0}$ & $1.4 \times 10^{0}$ & $4.6 \times 10^{-1}$ & $4.0 \times 10^{-1}$ & $2.2 \times 10^{\circ}$ & $4.9 \times 10^{0}$ \\
\hline Total & $3.2 \times 10^{3}$ & $1.1 \times 10^{2}$ & $7.5 \times 10^{1}$ & $1.5 \times 10^{3}$ & $1.7 \times 10^{3}$ & $4.8 \times 10^{3}$ \\
\hline
\end{tabular}




\section{Chapter 5. Dose Consequences}

This chapter describes the health impacts associated with exposure to radioactive material released from SRS. 
This page intentionally left blank 


\section{Relationship of Dose to Risk and Health Effects}

\section{lonizing Radiation}

Ionizing radiation is radiation that has enough energy to remove electrons from the atoms through which it passes. The interaction of ionizing radiation with biological systems can induce a series of chemical reactions that can cause permanent changes in the genetic material of cells. These changes (mutations) may cause abnormal functioning within the cell or may lead to cell death.

The nature of radiation-induced cellular changes depends on the magnitude of the dose and the rate at which it is received. For the low doses and dose rates encountered in the environment from SRS releases, the most significant potential effect is cancer induction. This is believed to be a stochastic effect (i.e., an increase in dose increases the probability of the effect, but the severity of the effect is independent of the dose).

A characteristic of stochastic risks is the absence of a threshold. In other words, it is conceivable that any dose of radiation, no matter how small, might give rise to a cancer. On the other hand, there is no way to be certain that a given dose, no matter how large, will cause a cancer in an individual.

In recent years, many scientists have begun to realize that there is little evidence of radiation effects at individual doses of $10 \mathrm{rem}$ or less. The Health Physics Society has taken the position that there should be no assessments of risk for low doses since "zero health effects is the most likely outcome" (Mossman 1996).

In this document, health effects will be estimated, but the reader should realize that the cancer deaths calculated may be a gross overestimate and that there may be no health effects at all.

\section{Cancer Risk Estimates for Atmospheric Releases}

The most comprehensive estimates of cancer induction by exposure to ionizing radiation come from studies of the atomic bomb survivors at Hiroshima and Nagasaki. Less-definitive studies include those of medical patients exposed to therapeutic and diagnostic radiation. Studies of laboratory animals have increased the understanding of dose-effect relationships. The International Commission on Radiological Protection (ICRP) has evaluated all these studies and concluded that the best estimate of lifetime risk of fatal cancer for members of the general population is approximately 500 cases per $1,000,000$ person-rem (ICRP 1991). This is equivalent to one case per 2,000 person-rem.

"Maximum" individuals are hypothetical persons who live at the SRS boundary and subsist on diets of locally produced milk, meat, and vegetables. No such individuals are known to exist. Nevertheless, if one examines the case of the maximally exposed adult individual living continuously at the Site perimeter throughout the period 1954 through 1996, the cumulative effective dose equivalent from atmospheric releases of radionuclides has been estimated at 77 mrem. This value is the upper bounding case and made a minor contribution to the overall dose received during that time period.

A person living in the Central Savannah River Area (CSRA) received an effective dose of approximately 12,700 mrem from exposure to natural sources of radioactivity and an additional 2,800 mrem 'from medical practices and various consumer products during the same 43-year period (WSRC 1994). Therefore, the cumulative dose contribution to this individual from SRS atmospheric releases of radionuclides is about $0.5 \%$ of that received from sources unrelated to SRS.

Because the contribution of SRS releases of radionuclides to any individual's total radiation dose is so small, it is necessary to pool the radiation exposures from a given population if an assessment of potential health risks is desired. The population dose within an $80-\mathrm{km}$ radius is the figure of merit frequently used to make such an assessment.

The population doses from atmospheric releases reported in Table 4-29 are based on 1980 census data $(555,100$ people within $80 \mathrm{~km})$ and current meteorological and dose factor data. If it is assumed that this population has lived in the SRS vicinity throughout the period of Site operations, the total collective effective dose received by the population through 1996 would be 3,200 person-rem.

The risks associated with this collective dose are quite small. The risk estimate using ICRP factors for the 
number of excess fatal cancers potentially induced by a collective dose of 3,200 person-rem is 1.6 . Conversely, in the same population, at the current fatal cancer frequency of $20 \%$ (NRC 1990), there will be about 110,000 fatal cancers from all other causes. Therefore, it is impossible to demonstrate that a relationship exists between any of the cancer deaths occurring in this population and the releases of radionuclides to the atmosphere.

\section{Cancer Risk Estimates for Liquid Releases}

The maximally exposed individual for liquid releases lives on the Savannah River downriver of SRS, drinks untreated water from the river, eats fish caught from the river and boats and swims in the river. The total dose to this hypothetical individual from 1954 through 1966 was 140 mrem. This can be compared with the dose from natural sources of radioactivity, medical practices, and consumer products listed above. The cumulative dose contribution to this individual from SRS liquid releases of radionuclides is $0.9 \%$ of that received from sources unrelated to SRS.

Collective or population doses to residents who drink Savannah River water, eat fish from the river, and eat saltwater invertebrates from the Savannah River estuary are reported in Tables 4-33 through 4-39.

Drinking water doses for users of the Beaufort-Jasper (50,000 customers) and Port Wentworth, (15,000 effective consumers) water treatment plants also have been estimated. Different terminology is used to describe the two populations to reflect the difference in their compositions (Hamby 1991). The Beaufort-Jasper plant services residential areas and therefore provides full-scale domestic water service. The Port Wentworth facility serves a commercial complex in which contact with treated Savannah River water is limited to industrial workers who consume tap water.

If the cumulative effective doses received by both water treatment plant populations are summed, the collective dose equivalent is about 190 person-rem. Using the ICRP nominal risk factor, the predicted impact of this collective dose is an estimated 0.1 excess fatal cancers in a population of 65,000 people-13,000 of whom, at the current fatal cancer rate-are projected to succumb to cancer from all other sources.

The total population dose for liquid releases is the sum of the dose from the water treatment plant pathway (190 person-rem, 65,000 people) plus the dose due to other liquid pathways such as fish (1,500 person-rem, 555,100 people). The collective dose equivalent is 1,700 person-rem distributed among 620,100 people. From this dose, the nominal risk factor predicts 0.9 fatal cancers in a population of 620,100 people $-124,000$ of whom will die of cancer from other sources.

It is of interest to note that the employees of the Savannah River Site have been occupationally exposed to about 63,000 person-rem of radiation (Taylor 1995). Most of these employees live in the vicinity of SRS and are included in the populations discussed above. Their radiation exposure dwarfs the environmental exposure and will make an epidemiological study of the effects of environmental radiation exposure meaningless.

\section{Comparisons of Doses Near SRS with Applicable Regulations}

\section{Atmospheric Releases}

The two highest hypothetical annual effective doses received by the maximally exposed individual because of atmospheric releases of radionuclides from SRS were 11 mrem in 1955 and 14 mrem in 1956. All other annual doses were well below 10 mrem. The current DOE and EPA annual limit for dose to members of the public because of atmospheric releases is 10 mrem (DOE 1990; EPA 1989). This limit did not exist in 1955 and 1956.

\section{Liquid Releases}

Radionuclide doses from drinking water sources are evaluated based on the DOE and EPA annual drinking water standard of 4 mrem (DOE 1990; EPA 1977). At no time during Site operations has a drinking water dose from SRS releases to the Savannah River exceeded $1.0 \mathrm{mrem}$. The maximum dose was $0.8 \mathrm{mrem}$ for Port Wentworth in 1963. 


\section{Summary of Dosimetric Impacts}

References for Chapter 5

The overall radiological impact of SRS radionuclide releases (1954 through 1996) on the offsite population can be characterized by a total dose of 4,800 person-rem distributed among 620,100 people. During this same period, however, the population received a dose of approximately 63,000 person-rem from occupational exposure at the SRS and 9,600,000 person-rem from other sources of ionizing radiation in the environment.

Table 5-1. SRS Occupational Doses, 1952-1994

\begin{tabular}{|c|c|c|c|}
\hline Year & $\begin{array}{c}\text { External and } \\
\text { Tritium } \\
\text { (per-rem) }\end{array}$ & $\begin{array}{c}\text { Internal } \\
\text { (per-rem) }\end{array}$ & $\begin{array}{c}\text { Total } \\
\text { (per-rem) }\end{array}$ \\
\hline 1952 & 21 & 1 & 22 \\
\hline 1953 & 43 & 0 & 43 \\
\hline 1954 & 70 & 24 & 94 \\
\hline 1955 & 400 & 208 & 608 \\
\hline 1956 & 930 & 335 & 1265 \\
\hline 1957 & 1400 & 46 & 1446 \\
\hline 1958 & 1788 & 41 & 1829 \\
\hline 1959 & 1687 & 72 & 1759 \\
\hline 1960 & 3057 & 214 & 3271 \\
\hline 1961 & 2118 & 45 & 2163 \\
\hline 1962 & 2265 & 210 & 2475 \\
\hline 1963 & 2253 & 32 & 2285 \\
\hline 1964 & 2937 & 81 & 3018 \\
\hline 1965 & 2369 & 94 & 2463 \\
\hline 1966 & 2128 & 54 & 2182 \\
\hline 1967 & 2761 & 217 & 2978 \\
\hline 1968 & 2420 & 134 & 2554 \\
\hline 1969 & 2790 & 188 & 2978 \\
\hline 1970 & 2390 & 280 & 2670 \\
\hline 1971 & 2401 & 101 & 2502 \\
\hline 1972 & 1711 & 67 & 1778 \\
\hline 1973 & 1488 & 74 & 1562 \\
\hline 1974 & 1370 & 81 & 1451 \\
\hline 1975 & 1169 & 147 & 1316 \\
\hline 1976 & 1176 & 24 & 1200 \\
\hline 1977 & 1173 & 522 & 1695 \\
\hline 1978 & 1142 & 75 & 1217 \\
\hline 1979 & 1172 & 58 & 1230 \\
\hline 1980 & 1211 & 69 & 1280 \\
\hline 1981 & 1229 & 133 & 1362 \\
\hline 1982 & 1138 & 107 & 1245 \\
\hline 1983 & 1127 & 29 & 1156 \\
\hline 1984 & 1077 & 28 & 1105 \\
\hline 1985 & 1141 & 46 & 1187 \\
\hline 1986 & 1130 & 102 & 1232 \\
\hline 1987 & 962 & 40 & 1002 \\
\hline 1988 & 897 & 40 & 937 \\
\hline 1989 & 807 & 10 & 817 \\
\hline 1990 & 722 & 11 & 733 \\
\hline 1991 & 429 & 20 & 449 \\
\hline 1992 & 325 & 1 & 326 \\
\hline 1993 & 262 & 1 & 263 \\
\hline 1994 & 313 & 1 & 314 \\
\hline Total & 59399 & 4063 & 63462 \\
\hline
\end{tabular}

U. S. Department of Energy, 1990, Radiation Protection of the Public and Environment, DOE Order 5400.5, Washington, $\mathrm{DC}$.

U.S. Environmental Protection Agency, 1977, National Interim Primary Drinking Water Regulations, EPA 570/976-003, Washington, DC.

U. S. Environmental Protection Agency, 1989, National Emission Standards for Emissions of Radionuclides Other than Radon from Department of Energy Facilities, 40 CFR, Part 61, Subpart H, Washington, DC.

Hamby, D.M., 1991, Land and Water Use Characteristics in the Vicinity of the Savannah River Site (U), WSRC-RP91-17, Westinghouse Savannah River Company, Aiken, South Carolina.

ICRP, 1991, International Commission on Radiological Protection. Risks Associated with lonizing Radiations, Oxford: Pergamon Press; ICRP Vol. 22, No. 1.

Mossman, K.L., M. Goldman, F. Masse, W.A. Mills, K.J. Schiager, and R.J. Vetter, "Health Physics Society Position Statement-Radiation Risk in Perspective," The Health Physics Society's Newsletter, XXIV:3, March 1996.

National Research Council, Committee on the Biological Effects of Ionizing Radiation (BEIR V), 1990, Health Effects of Exposure to Low Levels of Ionizing Radiation, Washington, D.C., National Academy Press.

Taylor, G.A., K.W. Crase, T.R. La Bone, and W.H. Wilkie, 1995, A History of Personnel Radiation Dosimetry at the Savannah River Site, WSRC-RP-95-234, Westinghouse Savannah River Company, Aiken, South Carolina.

WSRC, 1994, Savannah River Site Environmental Report for 1993, Summary Pamphlet, WSRC-TR-94-076, p. 6, Westinghouse Savannah River Company, Aiken, South Carolina. 


\section{Additional Reading}

The following documents in the Radiological Assessment Program series are available to the public from

\author{
National Technical Information Service \\ U.S. Department of Commerce \\ 5285 Port Royal Road \\ Springfield, VA 22161
}

Assessment of Activation Products in the Savannah River Site Environment WSRC-TR-95-0422

Assessment of Radiocarbon in the Savannah River Site Environment WSRC-TR-93-215

Cesium in the Savannah River Site Environment WSRC-RP-92-250

Also published in Health Phys. 67(3):233-244; 1994

Assessment of Selected Fission Products in the Savannah River Site Environment WSRC-TR-96-0220

Radioiodine in the Savannah River Site Environment WSRC-RP-90-424-2

Assessment of Mercury in the Savannah River Site Environment WSRC-TR-94-0218-ET

Assessment of Neptunium, Americium, and Curium in the Savannah River Site Environment WSRC-TR-97-00266

Assessment of Noble Gases in the Savannah River Site Environment WSRC-TR-95-219

Assessment of Plutonium in the Savannah River Site Environment WSRC-RP-92-879, Rev 1

Also published in Health Phys. 71(3):290-299; 1996
Assessment of Strontium

in the Savannah River Site

Environment

WSRC-RP-92-984

Assessment of Technetium

in the Savannah River Site Environment

WSRC-TR-93-217

Assessment of Tritium

in the Savannah River Site

Environment

WSRC-TR-93-214

Uranium in the Savannah River Site Environment WSRC-RP-92-315 


\section{DISTRIBUTION:}

SAVANNAH RIVER SITE

U.S. Department of Energy/Savannah River Field Office

D. Hannah (60)

703-46A

Westinghouse Savannah River Company

$\begin{array}{ll}\text { R.P. Addis } & \text { 773-A } \\ \text { L. Bagwell } & 730-2 \mathrm{~B} \\ \text { D.M. Beals } & 735-\mathrm{A} \\ \text { D.T. Bignell } & 742-\mathrm{A} \\ \text { A.L. Boni } & 773-\mathrm{A} \\ \text { P.D. Campbell } & 705-\mathrm{A} \\ \text { T.L. Capeletti } & 773-41 \mathrm{~A} \\ \text { W.H. Carlton (80) } & 773-42 \mathrm{~A} \\ \text { G. Clare } & 704-\mathrm{C} \\ \text { J.R. Cook } & 773-43 \mathrm{~A} \\ \text { J.W. Cook } & 742-\mathrm{A} \\ \text { J.C. Corey } & 773-41 \mathrm{~A} \\ \text { K.W. Crase } & 735-\mathrm{A} \\ \text { M. Denham } & 773-42 \mathrm{~A} \\ \text { M. Dodgen } & 735-11 \mathrm{~A} \\ \text { M.A. Ebra } & 773-41 \mathrm{~A} \\ \text { L. Eldridge } & 742-\mathrm{A} \\ \text { W.A. Emel } & 735-\mathrm{A} \\ \text { P.D. Fledderman } & 735-11 \mathrm{~A} \\ \text { L.A. Geary } & 735-11 \mathrm{~A} \\ \text { J.B. Gladden } & 773-42 \mathrm{~A} \\ \text { D.E. Gordon } & 742-\mathrm{A} \\ \text { J.E. Halverson } & 735-\mathrm{A} \\ \text { D.W. Hayes } & 735-\mathrm{A} \\ \text { L.A. Haselow } & 730-2 \mathrm{~B}\end{array}$

J.D. Heffner

D.D. Hoel

E.W. Holtzscheiter - 773-A

G.T. Jannik 773-42A

G.F. Jernigan 730-2B

N.D. Johnson 730-4B

M.V. Kantelo 735-A

R. Lorenz 735-16A

D.K. Martin 730-2B

H.L. Martin - 707-C

L.C. Martin 742-A

D.B. Moore-Shedrow 773-A

C.E. Murphy, Jr. $\quad 773-42 A$

R.L. Nichols 773-42A

J.V. Odum 742-A

L.M. Papouchado 773-A

J.B. Pickett 730-M

A.A. Simpkins 773-42A

H.J. Stafford 730-4B

D.E. Stephenson 730-2B

D.M. Tuck

773-42A

E.L. Wilhite 773-43A

F.B. Williams 730-4B

W.G. Winn 735-A

S. Wood 773-A

\section{Savannah River Ecology Laboratory}

$\begin{array}{ll}\text { P. Bertsch } & 737-A \\ \text { S.B. Clark } & 737-A \\ \text { T. Hinton } & 737-A \\ \text { M.C. Newman } & 737-A\end{array}$

J.E. Pinder

737-A

737-A

737-A

C.L. Strojan

737-A

\section{EXTERNAL}

Environmental Advisory Committee c/o C.E. Murphy, Jr., 773-42A (6)

J.E. Till, Neeses, SC 